First published in:

\title{
Periodic nanostructures for photonics
}

\author{
K. Busch ${ }^{\mathrm{a}, *}$, G. von Freymann ${ }^{\mathrm{b}}$, S. Linden ${ }^{\mathrm{b}}$, S.F. Mingaleev ${ }^{\mathrm{a}, \mathrm{c}}$, L. Tkeshelashvili $^{\mathrm{a}}$, \\ M. Wegener ${ }^{\mathrm{d}}$ \\ a Institut für Theoretische Festkörperphysik, Universität Karlsruhe (TH), D-76131 Karlsruhe, Germany \\ ${ }^{\mathrm{b}}$ Institut für Nanotechnologie, Forschungszentrum Karlsruhe in der Helmholtz-Gemeinschaft, D-76021 Karlsruhe, Germany \\ ${ }^{\mathrm{c}}$ Bogolyubov Institute for Theoretical Physics of NASU, $03680 \mathrm{Kiev}$, Ukraine \\ ${ }^{\mathrm{d}}$ Institut für Angewandte Physik, Universität Karlsruhe (TH), D-76131 Karlsruhe, Germany
}

Accepted 13 February 2007

Available online 5 March 2007

editor: G.I. Stegeman

\begin{abstract}
Periodic nanostructures in photonics facilitate a far-reaching control of light propagation and light-matter interaction. This article reviews the current status of this subject, including both recent progress and well-established results. The primary focus is on the basic physical principles and potential applications associated with the existence of Bragg scattering, photonic band structures, and engineered effective-medium properties in periodic dielectric and metallo-dielectric systems. In addition, we discuss advantages and limitations of various theoretical and numerical approaches as well as of those fabrication techniques that have specifically been developed for this field.
\end{abstract}

(C) 2007 Elsevier B.V. All rights reserved.

PACS: 42.25.-p; 42.50.-p; 42.65.-k; 42.70.Qs; 42.82.-m; 71.36.+c

\section{Contents}

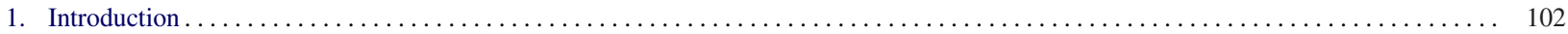

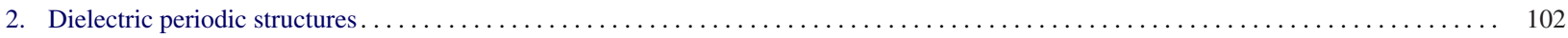

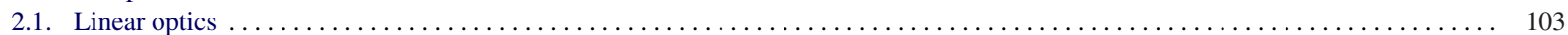

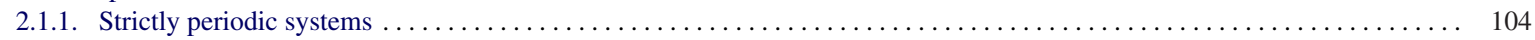

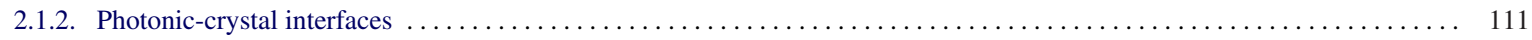

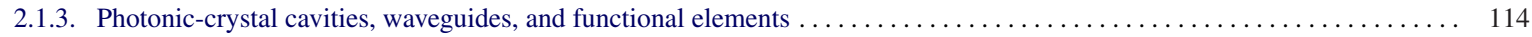

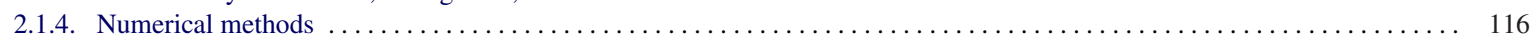

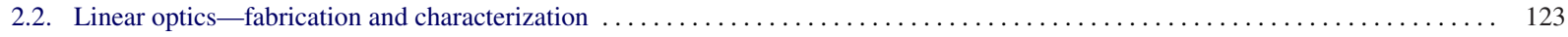

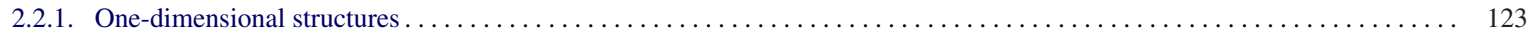

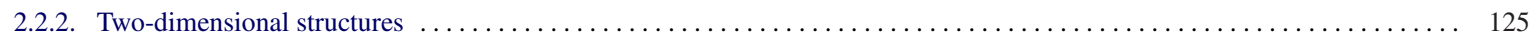

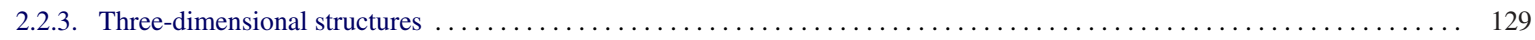

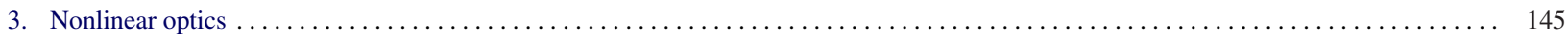

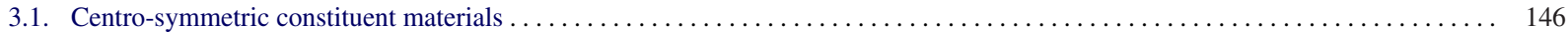

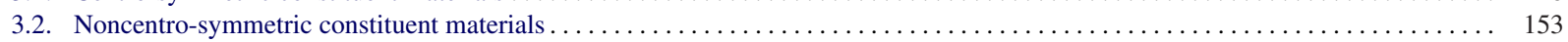

* Corresponding author. Fax: +497216087040.

E-mail address: kurt@tkm.physik.uni-karlsruhe.de (K. Busch). 


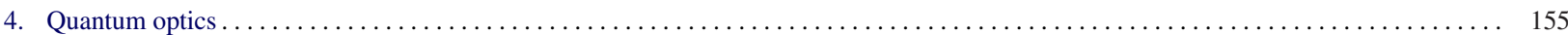

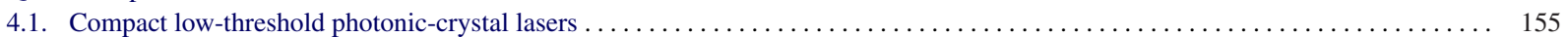

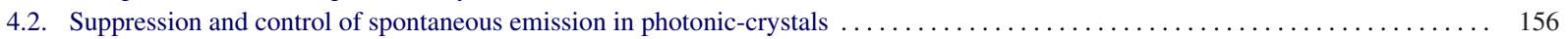

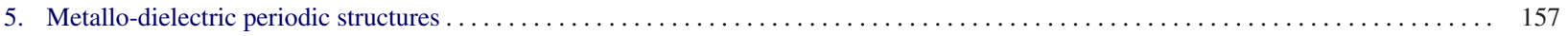

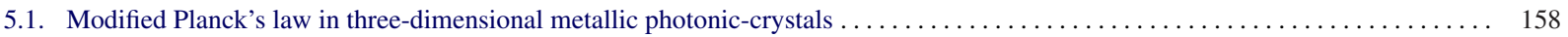

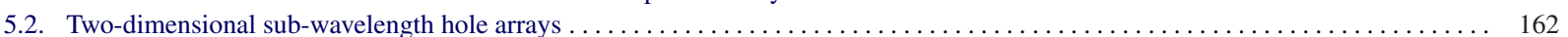

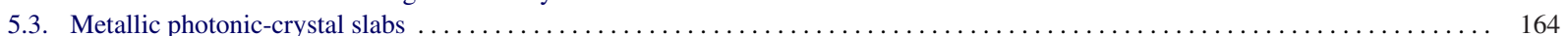

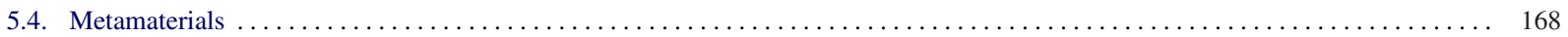

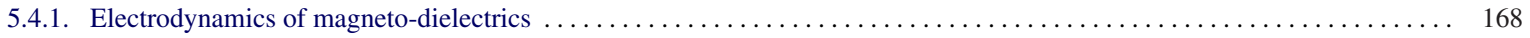

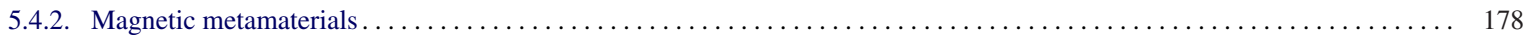

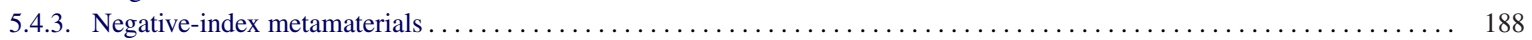

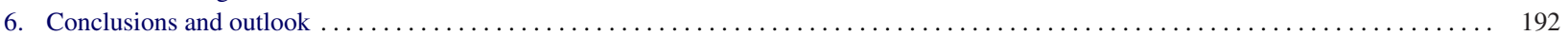

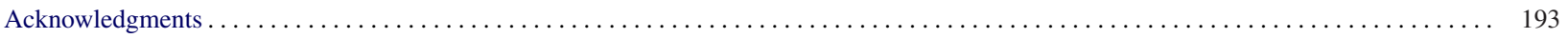

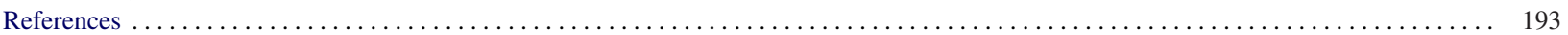

\section{Introduction}

With the introduction of the photon by Albert Einstein in 1905 and the subsequent invention of the Ruby laser by Theodore Harold Maiman in 1960, optics turned into photonics. This immediately triggered a revolution in many fields such as for example telecommunications, atomic/molecular spectroscopy, nonlinear optics, and-through advanced optical imaging and manipulation techniques-in biology. The future potential of optics and photonics is tremendous; hence, some have already called the emerging 21 st century "the century of the photon". Future progress in all of the above areas sensitively relies on the availability of novel and/or improved optical crystals, materials, and structures.

Over the last decade, the steady progress regarding our ability to fabricate photonic nanostructures has led to a rich variety of different one-, two-, and three-dimensional dielectric and/or metallic periodic structures. They exhibit qualitatively novel and fascinating linear-optical, nonlinear-optical and quantum-optical properties-providing an unprecedented control of light propagation and light-matter interaction. Some of this experimental work has been stimulated directly by revolutionary theoretical concepts such as, e.g., three-dimensional photonic-band-gap (PBG) materials or artificial magnetism at optical frequencies in metamaterials. In turn, experiments stimulate refined theoretical modeling as well as new concepts. Thus, experiment and theory cannot and will not be separated from one another here.

The present article is a snapshot of this vibrant and rapidly emerging field. Some sub-areas have reached a certain level of maturity, others are still exploding. Generally, the field has become quite broad and it is impossible to cover "everything". For example, we explicitly exclude photonic-crystal fibers, which shall be covered in a separate review in this journal. Furthermore, we restrict ourselves to the regime of photonics, i.e., structures aiming at microwave or far-infrared frequencies are not addressed experimentally in detail- though selected references are given. Whenever extensive reviews of sub-areas already exist, we avoid repetition and give reference to them. The emphasis of the present review is on structuring the vast amount of scientific material and on discussing important underlying physical principles. Regarding the latter, our guideline is Albert Einstein's quote: "Everything should be made as simple as possible, but not simpler."

\section{Dielectric periodic structures}

In the absence of free charges and currents, Maxwell's equations in continuous media read

$$
\begin{aligned}
& \nabla \times \vec{E}(\vec{r}, t)=-\frac{\partial}{\partial t} \vec{B}(\vec{r}, t), \quad \nabla \cdot \vec{D}(\vec{r}, t)=0, \\
& \nabla \times \vec{H}(\vec{r}, t)=+\frac{\partial}{\partial t} \vec{D}(\vec{r}, t), \quad \nabla \cdot \vec{B}(\vec{r}, t)=0 .
\end{aligned}
$$


One furthermore has the constitutive relations

$$
\begin{aligned}
& \vec{D}(\vec{r}, t)=\epsilon_{0} \vec{E}(\vec{r}, t)+\vec{P}(\vec{r}, t), \\
& \vec{B}(\vec{r}, t)=\mu_{0} \vec{H}(\vec{r}, t)+\vec{M}(\vec{r}, t)
\end{aligned}
$$

with the polarization $\vec{P}$ and the magnetization $\vec{M}$.

The aim of most structures discussed in this review is to "mold the flow of light". The direction of the flow of light is determined by the Poynting vector $\vec{S}$ given by

$$
\vec{S}(\vec{r}, t)=\vec{E}(\vec{r}, t) \times \vec{H}(\vec{r}, t) .
$$

It is important to note that the direction of the Poynting vector is generally different from both, that of the group velocity $\vec{v}_{\text {group }}$ and the wave vector of light $\vec{k}$. The magnitude of $\vec{v}_{\text {group }}$ can exceed the vacuum speed of light $c_{0}$ and can even be infinitely large. Brillouin and Yeh [1-3] have shown that for the special case of linear lossless ${ }^{1}$ dielectric and/or magnetic materials, $\vec{v}_{\text {group }}$ and $\vec{S}$ point in the same direction. ${ }^{2}$ Finally, the light intensity $I(\vec{r}, t)$ is defined as the modulus of the cycle-averaged Poynting vector.

\subsection{Linear optics}

For nondispersive, isotropic, and linear media, ${ }^{3}$ the constitutive relations (2.3) and (2.4) can be recast into the form

$$
\vec{D}(\vec{r}, t)=\epsilon_{0} \epsilon(\vec{r}) \vec{E}(\vec{r}, t)
$$

and

$$
\vec{B}(\vec{r}, t)=\mu_{0} \mu(\vec{r}) \vec{H}(\vec{r}, t) .
$$

In this section, we restrict ourselves to nonmagnetic materials with magnetic permeability $\mu(\vec{r}) \equiv 1$. In this case, the spatially varying dielectric function $\epsilon(\vec{r})$ encodes all the information about the structured medium. The linearity of the medium allows us to construct the solutions of Maxwell's equations (2.1) and (2.2) through superimposing time-harmonic electric and magnetic fields,

$$
\vec{E}(\vec{r}, t)=\vec{E}(\vec{r}) \exp (-\mathrm{i} \omega t)+\text { c.c. }
$$

and

$$
\vec{H}(\vec{r}, t)=\vec{H}(\vec{r}) \exp (-\mathrm{i} \omega t)+\text { c.c. },
$$

respectively, where $\vec{E}(\vec{r}) \equiv \vec{E}_{\omega}(\vec{r})$ and $\vec{H}(\vec{r}) \equiv \vec{H}_{\omega}(\vec{r})$ are frequency-dependent functions. As a consequence, we may combine the two curl equations in (2.1) and (2.2) to yield wave equations in the frequency domain for, respectively, the magnetic and the electric fields:

$$
\begin{aligned}
& \nabla \times\left(\epsilon^{-1}(\vec{r}) \nabla \times \vec{H}(\vec{r})\right)=\frac{\omega^{2}}{c_{0}^{2}} \vec{H}(\vec{r}), \\
& \epsilon^{-1}(\vec{r}) \nabla \times \nabla \times \vec{E}(\vec{r})=\frac{\omega^{2}}{c_{0}^{2}} \vec{E}(\vec{r}) .
\end{aligned}
$$

Here, $c_{0}=1 / \sqrt{\epsilon_{0} \mu_{0}}=2.9979 \times 10^{8} \mathrm{~m} / \mathrm{s}$ is the vacuum speed of light. We emphasize that (2.10) and (2.11) have to be solved subject to the constraints of the div-equations in (2.1) and (2.2).

\footnotetext{
${ }^{1}$ For actual physical systems, the notion "lossless" means that the real part of the electric permittivity (magnetic permeability) is much larger than its imaginary part in the relevant spectral region.

${ }^{2}$ By construction, the energy velocity [4] is parallel to the Poynting vector. For periodic structures, e.g., for ordinary crystals, metamaterials, and photonic crystals, all quantities are understood as averaged over a unit cell as well as a cycle of light.

${ }^{3}$ Nondispersive media imply instantaneous temporal response, thus, $\epsilon$ and $\mu$ are constant. For anisotropic media, $\epsilon$ and $\mu$ have to be replaced by tensorial quantities.
} 


\subsubsection{Strictly periodic systems}

Periodic photonic structures with periods, i.e., lattice constants, comparable to the wavelength of light are commonly referred to as photonic crystals. In contrast to nonperiodic systems, the electromagnetic eigenmodes of photonic crystals can be cast into a photonic band structure. The existence of this multi-branch dispersion relation is the most important consequence of the Floquet-Bloch theorem. Owing to the phenomenon of Bragg reflection, certain stop bands open up for propagation in any given direction. These extremely rich dispersive properties of photonic crystals lead to highly unusual refractive properties, novel possibilities for realizing phase matching in nonlinear optical applications, and significant enhancements of light-matter interaction due to low group velocities. Moreover, in certain structures the stop bands may overlap so that for an entire frequency range propagation is forbidden irrespective of direction. These PBG materials may be regarded as the optical analog of ordinary semiconductors. Controlled "doping" of PBG materials provides a novel platform for the realization of ultra-compact functional elements. We will elaborate on this issue in Section 2.1.3.

Historically, the concept of three-dimensional PBG materials has independently been introduced by Yablonovitch and John in 1987 [5,6]. In 1990, extensive numerical calculations by Ho, Chan, and Soukoulis [7] have shown that certain structures with a diamond-like symmetry do indeed exhibit three-dimensional PBGs.

2.1.1.1. Floquet-Bloch theorem and band structure. Infinitely extended and strictly periodic photonic crystals are characterized by a spatially periodic arrangement of dielectric material as described by the dielectric function (or electric permittivity) $\epsilon(\vec{r})$. The actual description of such periodic structures proceeds via two distinct steps. First, the underlying Bravais lattice specifies the periodic array in which identical units of the structure are arranged. A $d$-dimensional Bravais lattice $\boldsymbol{R}$ consists of all points with a $d$-dimensional position vector $\vec{R}$ of the form $\vec{R}=\sum_{i=1}^{d} h_{i} \vec{a}_{i}$, where $h_{i}, i=1, \ldots, d$ run through all integer values and the set $\left\{\vec{a}_{i}, i=1, \ldots, d\right\}$ of primitive vectors $\vec{a}_{i}$ is a set of linearly independent vectors that span the lattice. Besides the obvious translational symmetries, the symmetry group of a Bravais lattice in general includes rotations, reflections, and inversions that map the lattice onto itself. The subset of symmetry operations that leave a particular point of the lattice fixed, is called the point group of the Bravais lattice. In three dimensions, there exist seven distinct point groups and it can be shown [8] that any symmetry operation of a Bravais lattice may be constructed by successive applications of translations through Bravais lattice vectors and point-group operations. Therefore, we can assert that in an ideal photonic crystal, the dielectric function $\epsilon(\vec{r})$ exhibits the translational symmetry of a Bravais lattice $\epsilon(\vec{r})=\epsilon(\vec{r}+\vec{R})$ for all $\vec{r} \in \boldsymbol{R}$.

Second, a $d$-dimensional volume of space that, when translated through all the vectors of a corresponding Bravais lattice, exactly covers the entire space, is known as a unit cell of the lattice. While there is no unique way of choosing a unit cell for a given Bravais lattice, the most common choice is the Wigner-Seitz cell which is defined as that region of space that is closer to the origin than to any other lattice point. As a geometrical object, the Wigner-Seitz cell exhibits the full symmetry of the Bravais lattice. However, depending on the actual arrangement of dielectric material within the Wigner-Seitz cell (the motif in the language of crystallography), the overall symmetry of a photonic crystal may differ from the symmetry of the underlying Bravais lattice. This greatly increases the number of distinct symmetry groups of photonic crystals. These symmetry groups are known as space groups and can again be classified through decomposing the symmetry operations $\hat{O}=(\hat{o}, \vec{\tau})$ into operations $\hat{o}$ that leave a particular point fixed and certain translation operations (represented by vectors $\vec{\tau}$ ). These translation vectors $\vec{\tau}$ such as the displacements along skew symmetry axes are not necessarily elements of the Bravais lattice $\boldsymbol{R}$. The set $\boldsymbol{O}=\left\{\hat{o}_{i}, i=1, \ldots, M\right\}$ of (nontranslational) symmetry operations that transform a given photonic crystal into itself while leaving one point fixed, is known as the crystallographic point group. In three dimensions, a systematic investigation of all possible Bravais lattices and all ways of reducing the symmetry of a spherical object at the center of the Wigner-Seitz cell, delivers 230 distinct space groups, which are classified according to 32 distinct crystallographic point groups [8]. In Section 2.2.3.3, we will employ these space groups for the actual fabrication of photonic crystals via holographic lithography.

The Fourier analysis of functions with the periodicity of a Bravais lattice such as the dielectric function $\epsilon(\vec{r})$ directly leads to the notion of the reciprocal (dual) lattice associated with a given Bravais (direct) lattice $\boldsymbol{G}$. The reciprocal lattice vectors $\vec{G} \in \boldsymbol{G}$ satisfy $\vec{G} \cdot \vec{R}=m 2 \pi$ (with integer $m$ ) for all Bravais lattice vectors $\vec{R}$. This implies [8] that the reciprocal lattice is itself a Bravais lattice and, consequently, any vector $\vec{G}$ of the reciprocal lattice may be written as $\vec{G}=\sum_{i=1}^{d} h_{i} \vec{b}_{i}$. Here, $h_{i}, i=1, \ldots, d$ run through all integer values and a canonical set $\left\{\vec{b}_{i}, i=1, \ldots, d\right\}$ of primitive vectors $\vec{b}_{i}$ is defined via the solution of the system of linear equation $\vec{b}_{i} \cdot \vec{a}_{j}=2 \pi \delta_{i j}, i, j \in\{1, \ldots, d\}$. The Wigner-Seitz 
cell of the reciprocal lattice is known as the first Brillouin zone (BZ). As the name suggests, one also defines higher BZs which are primitive unit cells of a different type that are used in the theory of electronic properties of solids. The usage of higher BZs in the context of photonic crystals has been minimal to date and we, subsequently, use the expression BZ synonymously with first BZ. Furthermore, we would like to emphasize that the BZ inherits rotational symmetries of the underlying Bravais lattice, i.e., the rotational symmetries of the photonic crystal's point group (and not the crystallographic point group!).

We are now in a position to formulate the central element in the theoretical analysis of photonic crystals: The Floquet-Bloch theorem asserts that the eigenmodes of the wave equation $(2.10)$, where $\epsilon(\vec{r})=\epsilon(\vec{r}+\vec{R})$ for all $\vec{R}$ in a Bravais lattice $\boldsymbol{R}$, can be chosen to have the form of a plane wave times a vectorial function with the periodicity of the Bravais lattice

$$
\begin{aligned}
& \vec{H}_{n \vec{k}}(\vec{r})=\exp (\mathrm{i} \vec{k} \cdot \vec{r}) \vec{u}_{n \vec{k}}(\vec{r}), \\
& \vec{u}_{n \vec{k}}(\vec{r})=\vec{u}_{n \vec{k}}(\vec{r}+\vec{R}), \quad \vec{R} \in \boldsymbol{R} .
\end{aligned}
$$

The wave vector $\vec{k}$ is restricted to the $\mathrm{BZ}$ of the corresponding reciprocal lattice and the (discrete) band index $n$ occurs because, for a given wave vector $\vec{k}$, there will be many independent eigenmodes (see below). This theorem takes advantage of the fact that the operator $\nabla \times((1 / \epsilon(\vec{r})) \nabla \times$.$) is a hermitian operator with respect to the standard$ scalar product $\left\langle\vec{F}_{1} \mid \vec{F}_{2}\right\rangle=\int \mathrm{d}^{d} r \vec{F}_{1}^{*}(\vec{r}) \cdot \vec{F}_{2}(\vec{r})$ for vector-valued functions $\vec{F}_{1}$ and $\vec{F}_{2}$ and commutes with the translation operations of the photonic crystal's underlying Bravais lattice $\boldsymbol{R}$. An analogous theorem with the same physical content may be derived for the wave equation (2.11) by noting that the operator $(1 / \epsilon(\vec{r})) \nabla \times \nabla \times$ is hermitian with respect to the modified scalar product $\left(\vec{F}_{1} \mid \vec{F}_{2}\right)=\int \mathrm{d}^{d} r \in(\vec{r}) \vec{F}_{1}^{*}(\vec{r}) \cdot \vec{F}_{2}(\vec{r})$. The actual proof of the Floquet-Bloch theorem closely follows the corresponding proofs in the case of electronic crystals [8] and can also be found in the photonic-crystal literature [9]. Therefore, instead of providing a formal proof, we discuss the profound consequences that follow from the Floquet-Bloch theorem:

(i) The Floquet-Bloch theorem identifies lattice-periodically modulated plane waves with vector $\vec{k}$ as the eigenmodes of a strictly periodic dielectric system. Therefore, these Bloch functions may be regarded as a generalization of the familiar plane-wave basis of homogeneous media with infinitesimal translation symmetry to the present case of media with a discrete translational symmetry. The band index $n$ appears because for a given wave vector $\vec{k}$, there are many solutions to the wave equation (2.10). This follows from the fact that instead of (2.10), we could solve the corresponding equation for the lattice-periodic function $\vec{u}_{n \vec{k}}(\vec{r})$ which is obtained by combining the Floquet-Bloch theorem (2.12) with (2.10). For such an eigenvalue problem with periodic boundary conditions on the Wigner-Seitz cell, we expect to find an infinite family of solutions with discretely spaced eigenvalues labeled by the index $n$. Loosely speaking, this situation corresponds to the problem of higher harmonics associated with a vibrating string.

A more rigorous argument starts with the observation that the wave vector $\vec{k}$ in the Floquet-Bloch theorem can always be confined to lie with the BZ: Any wave vector $\vec{k}^{\prime}$ can be written as a sum $\vec{k}^{\prime}=\vec{k}^{*}+\vec{G}$ of a vector $\vec{G}$ of the reciprocal lattice and a wave vector $\vec{k}^{*}$ that does lie in the BZ. Since $\exp (\mathrm{i} \vec{G} \cdot \vec{r})$ is a lattice periodic function, we may absorb this factor into the function $\vec{u}_{n \vec{k}}(\vec{r})$, again leading to a discrete index $n$ that labels the reciprocal lattice vectors (or "higher harmonics"). The restriction of the wave vector to the BZ is known as "back-folding" of the infinite wave-vector space into the first BZ [8].

(ii) The restriction of the wave vector $\vec{k}$ to the first BZ implies that we can continue the dispersion relation $\omega_{n \vec{k}}$ and the Bloch functions $\vec{H}_{n \vec{k}}(\vec{r})$ to wave vectors $\vec{k}$ outside the BZ as we see fit. In view of the above discussion, the most common choice is to assign identical eigenfrequencies and eigenmodes to wave vectors differing by a reciprocal lattice vector $\vec{G}$ :

$$
\begin{aligned}
& \vec{H}_{n \vec{k}+\vec{G}}(\vec{r})=\vec{H}_{n \vec{k}}(\vec{r}), \\
& \omega_{n \vec{k}+\vec{G}}=\omega_{n \vec{k}} .
\end{aligned}
$$

The family of (in wave-vector space) continuous functions $\omega_{n \vec{k}}$ constitutes the dispersion relation of the strictly periodic system and is collectively referred to as the photonic band structure of the corresponding photonic crystal. 
(iii) Except for the special case of a frequency degeneracy, an individual eigenmode—a Bloch function- $\vec{H}_{n \vec{k}}(\vec{r})$ is determined up to an overall complex phase factor. This is to say that $\exp \left(\mathrm{i} \phi_{n \vec{k}}\right) \vec{H}_{n \vec{k}}(\vec{r})$ is just as good a Bloch function as $\vec{H}_{n \vec{k}}(\vec{r})$ and that all physical quantities should be independent under a phase transformation

$$
\vec{H}_{n \vec{k}}(\vec{r}) \rightarrow \exp \left(\mathrm{i} \phi_{n \vec{k}}\right) \vec{H}_{n \vec{k}}(\vec{r}) .
$$

The Bloch modes (2.12) and the corresponding eigenfrequencies, $\omega_{n \vec{k}}$, can be calculated numerically using several approaches. The simplest (and most often used one) is the so-called plane-wave approach based on the expansion of Bloch modes into a series of plane waves as we describe in more detail in Section 2.1.4.1.

Figs. 1 and 2 show examples of photonic band structures calculated for two different 2D photonic crystals composed of air holes in a dielectric host material, assuming that light propagates in the plane normal to the holes. We plot all results in terms of dimensionless frequency, $a / \lambda \equiv \omega a / 2 \pi c_{0}$, where $\lambda$ is the vacuum wavelength of light. In this case,

a

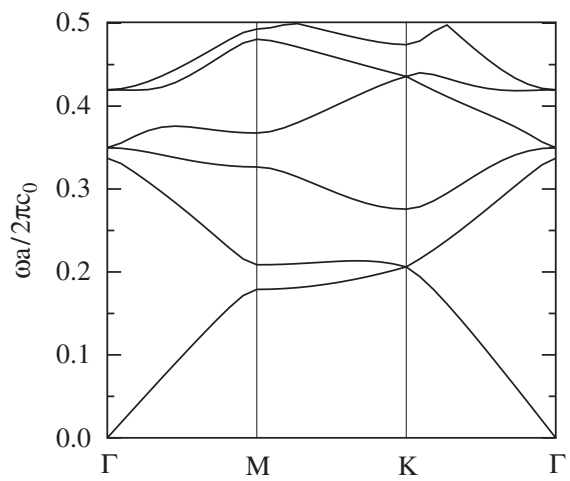

b

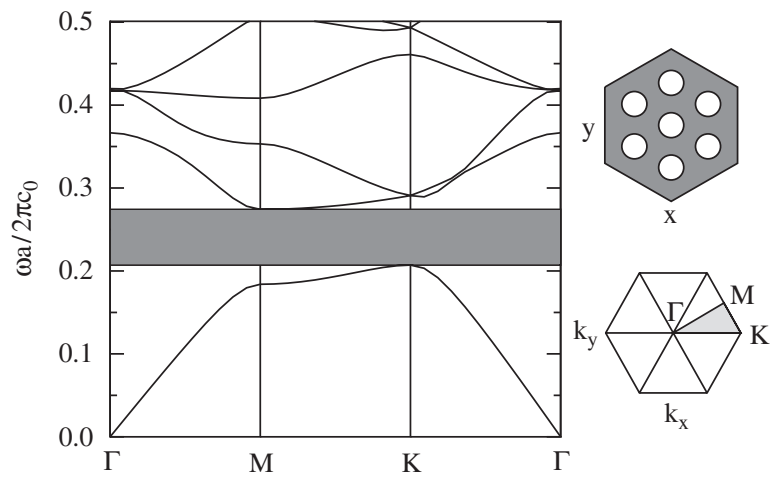

Fig. 1. Photonic band structure for (a) $E$-polarized and (b) $H$-polarized light in $2 \mathrm{D}$ photonic crystal created by a triangular lattice of air holes $\left(\epsilon_{1}=1\right)$ with the radius $r_{0}=0.3 a$ (where $a$ is the lattice period) in a dielectric with the permittivity $\epsilon_{2}=12$; the band gaps are hatched. The top right inset shows a cross-sectional view of the $2 \mathrm{D}$ photonic crystal. The bottom right inset shows the corresponding Brillouin zone, with the irreducible zone shaded.

a

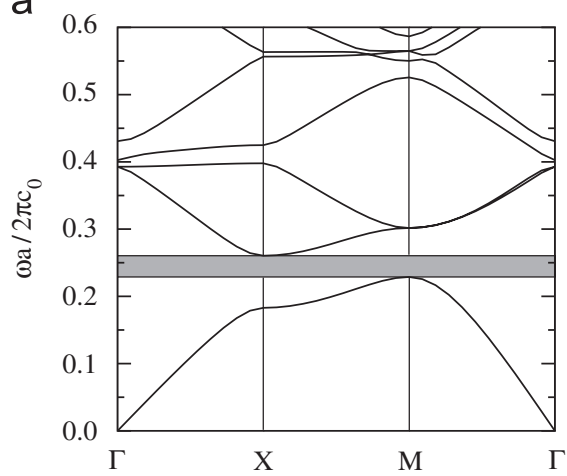

b

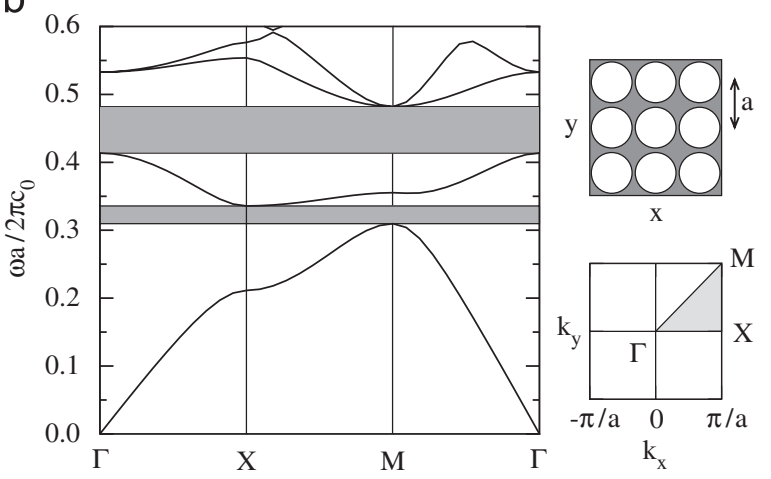

Fig. 2. Photonic band structure for (a) $E$-polarized and (b) $H$-polarized light in $2 \mathrm{D}$ photonic crystal created by a square lattice of air holes $\left(\epsilon_{1}=1\right)$ with the radius $r_{0}=0.45 a$ (where $a$ is the lattice period) in a dielectric with the permittivity $\epsilon_{2}=12$; the band gaps are hatched. The top right inset shows a cross-sectional view of the $2 \mathrm{D}$ photonic crystal. The bottom right inset shows the corresponding Brillouin zone, with the irreducible zone shaded. 


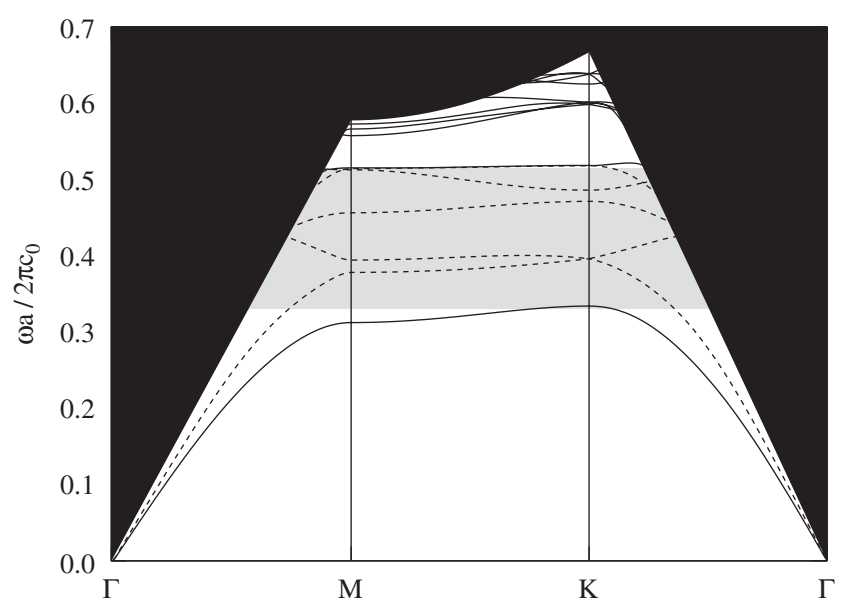

Fig. 3. Photonic band structure for a $2 \mathrm{D}$ photonic-crystal slab consisting of a planar waveguide $(\epsilon=12$, thickness $d=0.67 a)$ perforated with a hexagonal array of holes $\left(r_{0}=0.44 a\right)$. The shaded regions represent the leaky modes. $H$ - and $E$-polarized-like modes are depicted by solid and dashed lines, respectively. In this system, a photonic band gap for the confined $H$-polarized modes is indicated by the gray area.

all the Bloch modes can be conveniently separated into two sub-groups: The Bloch modes for which the electric field is parallel to the hole axis (we shall refer to such modes as to the $E$-polarized ones) and the Bloch modes for which the magnetic field is parallel to the holes axis ( $H$-polarized modes). Very often, it is tacitly assumed that the extrema of $\omega_{n \vec{k}}$ for every band $n$ are located on the high-symmetry lines of the first BZ. Therefore, it is usually sufficient to plot the photonic band structure along the high-symmetry lines of the first BZ shown in the bottom right insets of Figs. 1 and 2. However, this is correct only for a (rather large) class of photonic-crystal structures that exhibit sufficiently symmetric unit cells [10]. ${ }^{4}$ In the general case [10], a rigorous proof of the existence of photonic band gaps and the determination of their sizes requires the calculation of the density of states (see Section 2.1.1.2 and Figs. 4 and 5).

In the long-wavelength limit, i.e., for the first band close to the center of the BZ, the dispersion relations in Figs. 1 and 2 are very well approximated by straight lines. This means that one can introduce an effective refractive index. However, this refractive index depends on the polarization. For $E$-polarization, exact calculations find the naively expected result, namely the effective dielectric constant is given by the arithmetic volume average of the dielectric constants of the constituent materials, i.e.,

$$
\epsilon_{\mathrm{eff}}=(1-f) \epsilon_{1}+f \epsilon_{2},
$$

with the volume filling fraction $f$ of the material with dielectric constant $\epsilon_{2}$. For the parameters of Fig. 1(a), one obtains $\epsilon_{\text {eff }}=8.40$. The situation is rather different for the $H$-polarization. Here, an explicit expression cannot be given and we refer to [11]. For the parameters of Fig. 1(b), one obtains $\epsilon_{\text {eff }}=6.81$. In other words, the photonic crystal acts as a birefringent effective material (form birefringence). The corresponding 3D case has been discussed in Ref. [12]. We will come back to this long-wavelength limit of photonic crystals in the context of metamaterials in Section 5.4.

An example for a band structure of a 3D photonic crystal is shown in Fig. 5. 2D photonic-crystal slabs (see Fig. 3) conceptually lie in-between strictly 2D and 3D photonic crystals. In essence, a 2D photonic-crystal slab is a planar waveguide, with in-plane periodicity, e.g., a hexagonal array of holes. Guided wave propagation in the plane relies on index guiding in the third dimension. As a result, only certain photonic states are strictly confined. More precisely, out-coupling from the slab requires both, conservation of frequency and of the in-plane component of the wave vector. Thus, only states with a wave vector larger than the vacuum wave number at that frequency $\omega$ are strictly confined. In other words, these states are located outside the so-called light-cone defined by $|\vec{k}|=\omega / c_{0}$. To directly identify these states, the light has to be represented within the first BZ. This is illustrated in Fig. 3, where the black area highlights the continuum of so-called leaky modes, i.e., modes inside the light cone. If the

\footnotetext{
${ }^{4}$ We are indebted to Peter Kuchment for making available to us the results of Ref. [10] prior to publication.
} 
photonic-crystal slab is clad by a dielectric rather than vacuum, the light cone of the dielectric has to be used. For asymmetric cladding, the confined states need to be outside of both light cones simultaneously. For more details, we refer to Ref. [13].

Finally, we want to emphasize that the content of the Floquet-Bloch theorem and the concept of photonic band structures also applies to periodic arrangements of optically anisotropic materials. This opens the route to tunable photonic band structures [14,15]: The typically rather large porosity of high-index photonic crystals allows their infiltration with electro-optically tunable low-index materials such as liquid crystals and/or polymers. The largevolume fraction of optically anisotropic material makes the resulting composite systems highly efficacious for electrooptic tuning effects, thus potentially enhancing the technological utility of these composites over and above that of bulk anisotropic materials or conventional photonic crystals by themselves. In particular, photonic band structure computations have shown that controlling optical anisotropies allows for the opening and closing of complete threedimensional photonic band gaps in liquid crystal infiltrated PBG materials [14]. Later, the concept of tunable photonic band structures has been extended to tunable superrefractive effects [16] (see Section 2.1.2.1) as well as to tunable waveguiding structures and functional elements in photonic crystals [17-21] (see Section 2.1.3). In addition, liquidcrystal infiltrated photonic crystals may provide a novel form of (tunable) disorder [14] which—besides studying fundamental aspects of wave propagation such as Anderson localization-may find applications in the testing of random numbers $[22,23]$. To date, only bulk electro-optically tunable photonic-crystal structures using either liquid crystals or polymers have been realized experimentally [15,24-30] and limited tunability of the transmittance/reflectance has been demonstrated.

2.1.1.2. Density of states. In analogy to solid-state physics, the photonic dispersion relation $\omega_{n}(\vec{k})$ gives rise to a photonic density of states (DOS). In particular, spectral intervals of vanishing DOS represent photonic band gaps. In contrast to a determination on the basis of high-symmetry lines in the BZ, this definition is unambiguous. As the total number of photonic states is conserved, regions of vanishing or suppressed DOS lead to other regions with enhanced DOS with respect to vacuum. We will come back to effects based on suppressed DOS in Section 4 and to effects based on enhanced DOS in Section 5.1. Recall, that the $d$-dimensional photonic DOS, $N(\omega)$, is defined by "counting" all allowed states within an infinitesimally small frequency interval centered around frequency $\omega$

$$
N(\omega)=\sum_{n} \int_{B Z} \mathrm{~d}^{d} k \delta\left(\omega-\omega_{n}(\vec{k})\right) .
$$

For applications in the context of quantum optical experiments in photonic crystals, it is necessary to investigate not only the global DOS, but also the local DOS (LDOS) [31,32]. For instance, for an electric dipole emitter at position $\vec{r}$, the overlap matrix elements of the emitter's dipole moment with the eigenmodes (Bloch functions) of the photonic crystal determine its radiative properties (see Section 4). In this case, the LDOS becomes

$$
N(\vec{r}, \omega)=\sum_{n} \int_{B Z} \mathrm{~d}^{d} k\left|\vec{E}_{n \vec{k}}(\vec{r})\right|^{2} \delta\left(\omega-\omega_{n}(\vec{k})\right) .
$$

For an actual calculation, the integrals in (2.18) and (2.19) must be suitably discretized and one may again revert to the methods of electronic band structure calculations $[33,34]$. However, care has to be exerted when employing the symmetry properties of the Bloch functions in order to reduce the integration domain in (2.19) to an integration over the irreducible part of the BZ [35].

For nonperiodic systems such as finite-size photonic crystals, curvilinear photonic crystals [36] and photonic quasicrystals [37], the eigenmodes are generally not available so that the LDOS has to be obtained directly from the photonic Green's function [38] (see also Section 2.1.4.7)

$$
N(\vec{r}, \omega)=-\frac{2 \omega}{c_{0}^{3}} \epsilon(\vec{r}) \operatorname{Im}\left[\operatorname{Tr}\left(\mathbf{G}\left(\vec{r}, \vec{r}^{\prime}=\vec{r} ; \omega\right)\right)\right] .
$$

Here, $\operatorname{Tr}($.$) and \operatorname{Im}[$.$] denote, respectively, the trace of a matrix and the imaginary part of a complex number. For an$ infinite and defect-free photonic crystal, the Green's function $\mathbf{G}\left(\vec{r}, \vec{r}^{\prime} ; \omega\right)$ may be expanded into the corresponding Bloch functions (see Section 2.1.4.7) so that (2.20) reduces to (2.19). 
a

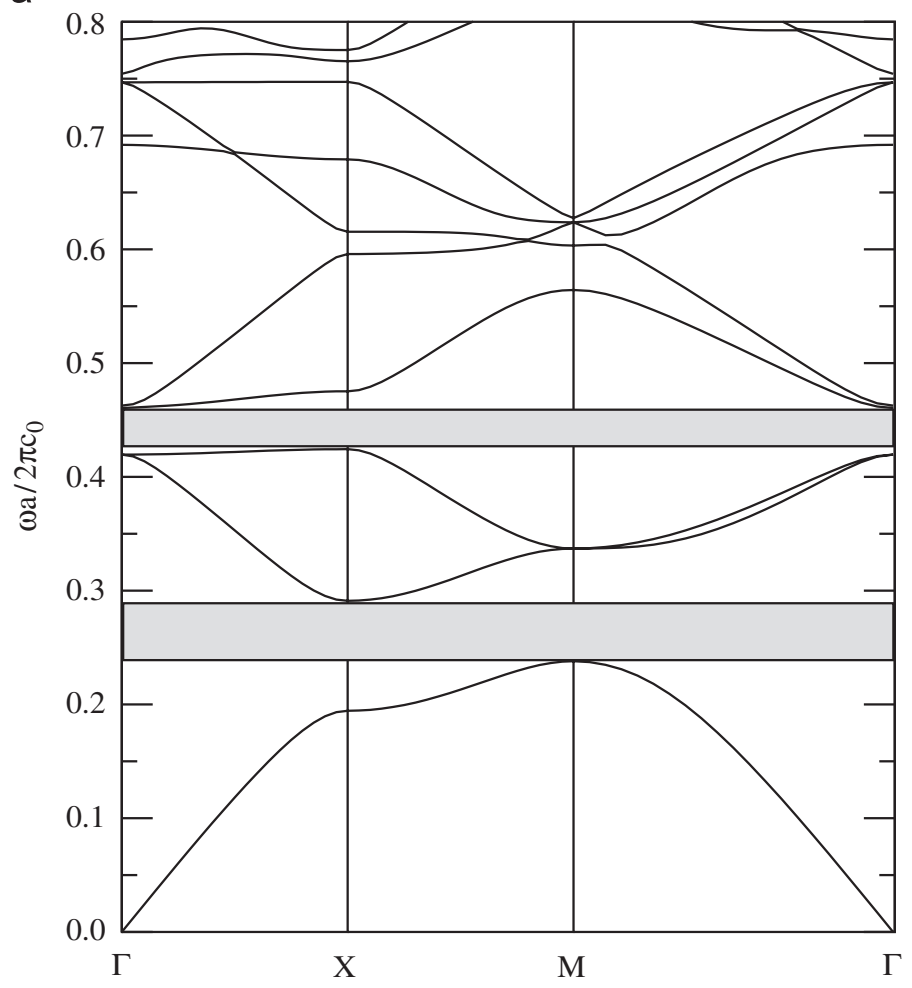

b

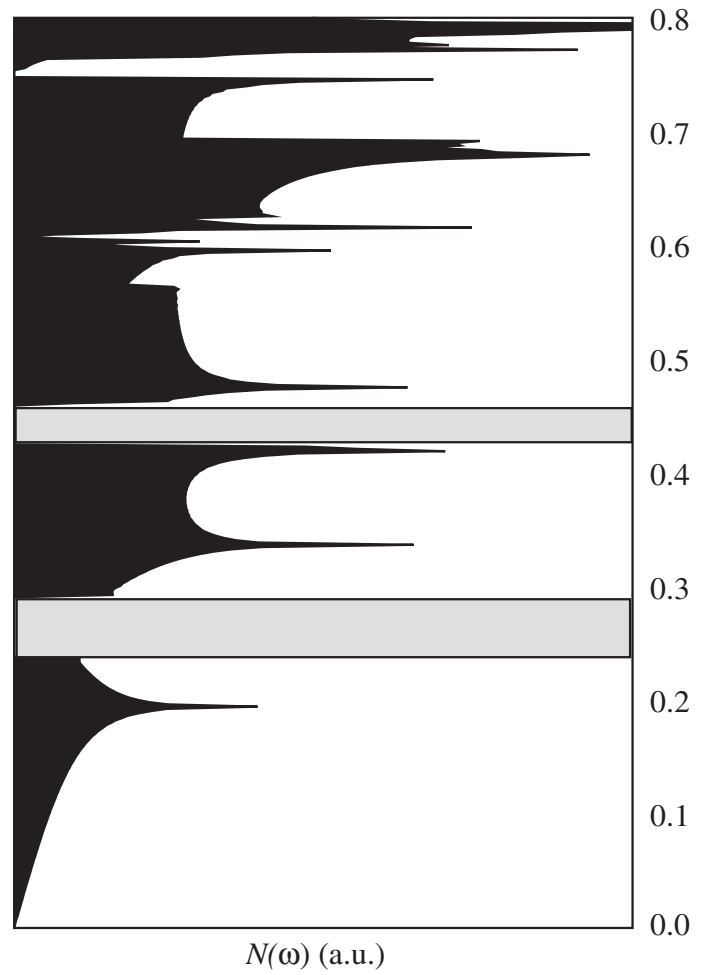

Fig. 4. 2D photonic band structure (a) and the corresponding DOS (b) for E-polarized light in a square lattice (lattice constant $a$ ) of cylindrical air holes of radius $r=0.475 a$ in dielectric with $\epsilon=12$. This photonic crystal exhibits a large fundamental photonic band gap extending from $\omega=0.238 \times 2 \pi c / a$ to $0.291 \times 2 \pi c / a$. A higher-order band gap extends from $\omega=0.425 \times 2 \pi c / a$ to $0.464 \times 2 \pi c / a$.

In Fig. 4, we display the 2D photonic band structure and DOS for $E$-polarized light in a photonic crystal composed of a square lattice of air holes (radius $r=0.475 a)$ in a dielectric matrix $(\epsilon=12)$.

Fig. 5(a) shows the band structure of a 3D photonic crystal, an inverse opal (see Section 2.2.3.2), (b) the corresponding 3D DOS. Note that the DOS is highly structured, both in 2D and 3D. This aspect will become important in the context of quantum optical effects (see Section 4).

2.1.1.3. Group velocity and group velocity dispersion. In order to understand pulse propagation in linear and nonlinear photonic crystals, it is necessary to obtain the group velocity as well as the group velocity dispersion (GVD) from the corresponding photonic band structure. In principle, this can be accomplished through numerical differentiations, but in particular for the GVD this becomes computationally involved and great care must be taken in order to avoid numerical instabilities specifically near or at points of degeneracies.

A significantly more efficient and accurate approach to the calculation of group velocities and GVD is provided by an adaptation of the so-called $\vec{k} \cdot \vec{p}$-perturbation theory of electronic band structure theory [40]. This is facilitated by combining the wave equation, (2.10), with the Floquet-Bloch theorem (2.12), to arrive at an equation of motion for the lattice periodic function $\vec{u}_{n \vec{k}}$

$$
\left(\hat{L}+\hat{l}_{\vec{k}}\right) \vec{u}_{n \vec{k}}(\vec{r})=\frac{\omega_{n \vec{k}}^{2}}{c_{0}^{2}} \vec{u}_{n \vec{k}}(\vec{r}),
$$


a

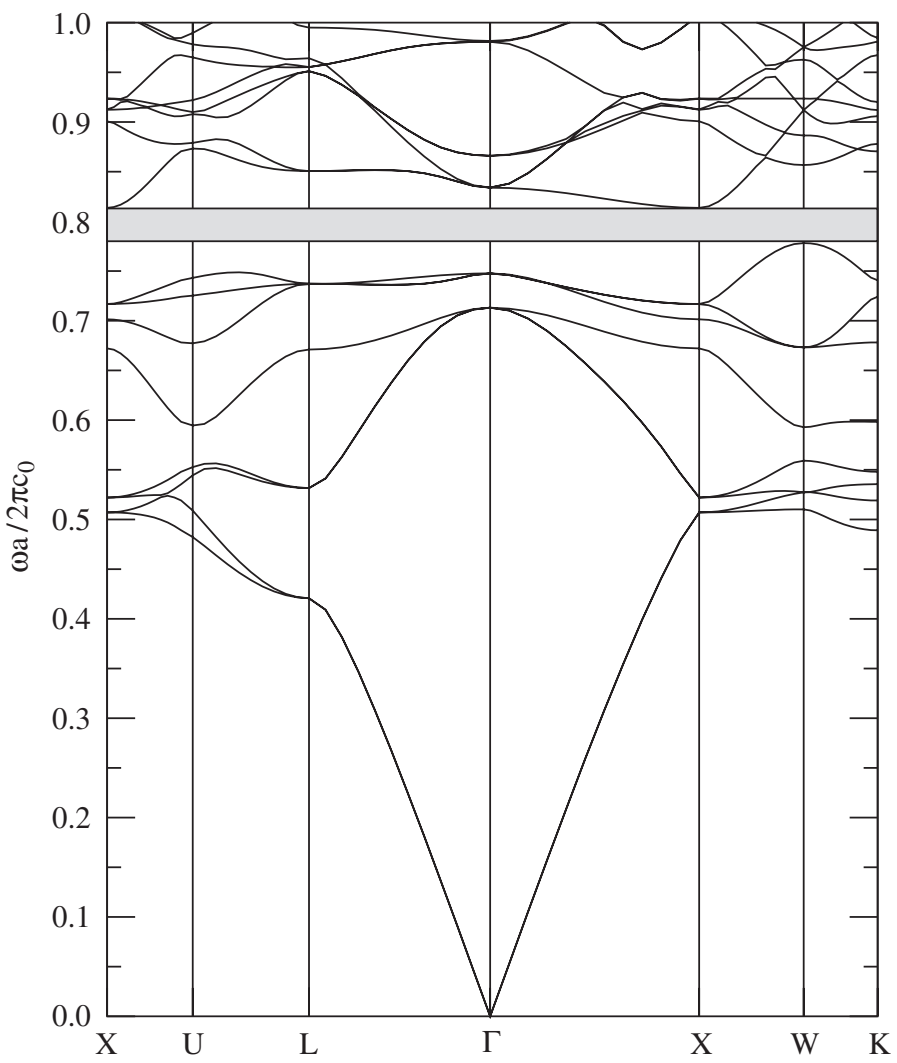

b

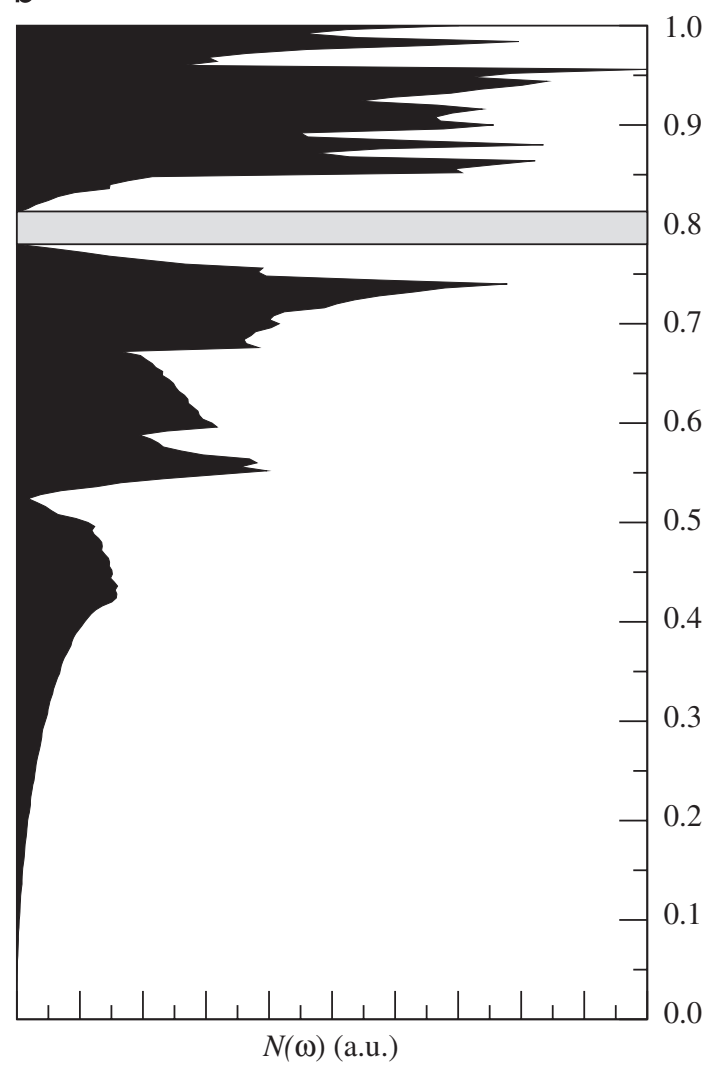

Fig. 5. (a) Band structure of a silicon inverse opal, (b) corresponding 3D DOS. After Ref. [39].

where the operators $\hat{L}$ and $\hat{l}_{\vec{k}}$ are defined according to

$$
\begin{aligned}
& \hat{L} \vec{u}_{n \vec{k}}(\vec{r})=\nabla \times\left(\frac{\nabla \times \vec{u}_{n \vec{k}}(\vec{r})}{\epsilon(\vec{r})}\right), \\
& \hat{l}_{\vec{k}} \vec{u}_{n \vec{k}}(\vec{r})=\nabla \times\left(\frac{\mathrm{i} \vec{k} \times \vec{u}_{n \vec{k}}(\vec{r})}{\epsilon(\vec{r})}\right)+\mathrm{i} \vec{k} \times\left(\frac{\nabla \vec{u}_{n \vec{k}}(\vec{r})+\mathrm{i} \vec{k} \times \vec{u}_{n \vec{k}}(\vec{r})}{\epsilon(\vec{r})}\right) .
\end{aligned}
$$

The $\vec{k} \cdot \vec{p}$-perturbation theory starts by writing (2.21) at a nearby point $\vec{k}^{\prime}=\vec{k}+\vec{q}$, where $\vec{q}$ is a wave vector whose absolute value $|\vec{q}| \ll \pi / a$ is much smaller than the extent of the BZ. Upon rewriting $\hat{L}+\hat{l}_{\vec{k}+\vec{q}}=\hat{L}+\hat{l}_{\vec{k}}+\hat{\delta} l_{\vec{k}, \vec{q}}$ with $\hat{\delta} l_{\vec{k}, \vec{q}}=\hat{l}_{\vec{k}+\vec{q}}-\hat{l}_{\vec{k}}$ we may regard $\hat{\delta} l_{\vec{k}, \vec{q}}$ as a small correction to the operator $\hat{L}+\hat{l}_{\vec{k}}$ and, therefore, may evaluate the "perturbed" eigenvalues $\omega_{n \vec{k}+\vec{q}}^{2} / c^{2}$ and corresponding "perturbed" eigenfunctions $\vec{u}_{n \vec{k}+\vec{q}}(\vec{r})$ through a perturbation series in $\vec{q}$ that utilizes the "unperturbed" eigenvalues $\omega_{n \vec{k}}^{2} / c^{2}$ and eigenfunctions $\vec{u}_{n \vec{k}}(\vec{r})$. A comparison with a Taylorexpansion of $\omega_{n \vec{k}+\vec{q}}$ around $\vec{k}$ reveals that the group velocity and GVD, respectively, can be obtained from the first and second orders in this perturbation series. The evaluation of the corresponding expressions in 1D [41], 2D [42], and 3D [43] can straightforwardly be done using standard band structure codes and rapid convergence is achieved without numerical instabilities. In addition, these expressions prove to be very valuable in the context of analytic calculations using multiple-scales techniques (see Section 3).

An example is shown in Fig. 6. The group velocities of bands 1, 3, and 5 exhibit extreme variations and may even become zero. The direction of the group velocity will be discussed in Section 2.1.2.1. This rich behavior may have applications in superrefractive phenomena (see Section 2.1.2.1) and classical nonlinear optics (see Section 3). 


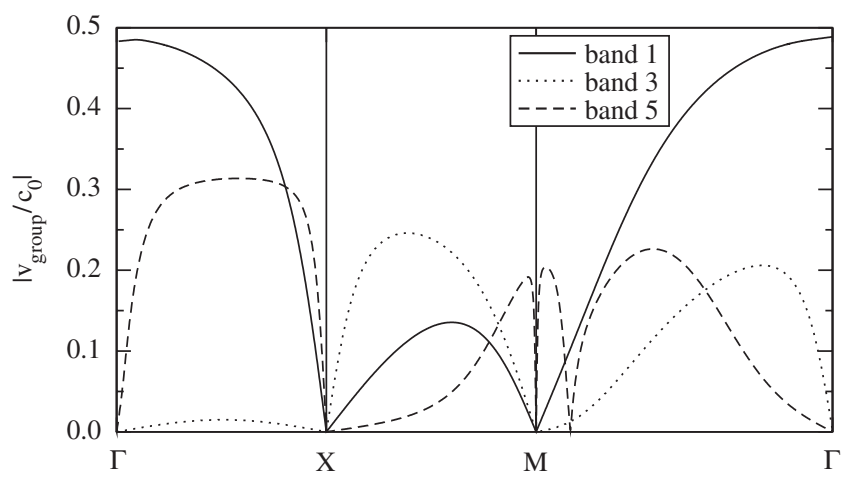

Fig. 6. Group velocities for bands 1, 3, and 5 of the model system shown in Fig. 4.

\subsubsection{Photonic-crystal interfaces}

Any actual photonic crystal is finite in size, revealing interfaces to the outside world. Interfaces give rise to refraction, diffraction, and to surface waves. Moreover, the symmetry of a photonic-crystal Bloch mode at the interface determines whether, e.g., an incident plane wave can actually couple to this mode.

2.1.2.1. Superrefractive effects. Inside a photonic crystal, the group-velocity vector $\vec{v}_{\text {group }}=\vec{\nabla}_{\vec{k}} \omega(\vec{k})$ is simply perpendicular to the iso-frequency surfaces $\omega(\vec{k})=$ const. $=\omega_{0}$ of the photonic crystal. These iso-frequency surfaces are in some sense analogous to Fermi surfaces in solid-state physics [8]. If $\omega$ increases (decreases) with increasing $|\vec{k}|$ along this direction, the group velocity vector points "uphill” (“downhill”). For lossles and purely dielectric materials, the group velocity vector is parallel to the Poynting vector (see Section 2). Thus, $\vec{v}_{\text {group }}$ indeed governs the flow of light in this case. While the iso-frequency surfaces of light in vacuum are simply spherical, the iso-frequency surfaces can be rather complex for actual photonic-crystal band structures. Furthermore, their shape and symmetry can strongly depend on the frequency $\omega_{0}$.

Let us consider light impinging from vacuum or air onto a photonic crystal, which is cut along a certain lattice plane. Under these conditions, the component of the incident wave vector of light parallel to the interface plane, $\vec{k}_{\|}$, is conserved modulo reciprocal lattice vectors $\vec{G}$. For simplicity, we consider the zeroth diffraction order, i.e., $\vec{G}=0$. $\vec{G} \neq 0$ would correspond to higher diffraction orders that may be evanescent. Furthermore, the incident frequency of light is conserved. Thus, for a given incident frequency of light $\omega_{0}$, one first has to select the corresponding isofrequency surface of the photonic crystal $\omega(\vec{k})=\omega_{0}$. Next, one has to find an intersection (or several) of the wave vector $\vec{k}$ for this $\vec{k}_{\|}$. Such intersections clearly only exist if $\left|\vec{k}_{\|}\right|$is not too large. Otherwise, light cannot couple into the photonic crystal, which is just a generalized version of the well-known phenomenon of total internal reflection. As a result of this procedure, $\vec{v}_{\text {group }}$ and $\vec{k}$ are generally not parallel. They can even point in opposite directions, i.e., $\vec{k} \cdot \vec{v}_{\text {group }}<0$. Let us consider a few examples.

Suppose we have the tight-binding-like (i.e., cos-like) band structure of a simple-cubic lattice with lattice constant $a$, i.e.,

$$
\omega(\vec{k})=\bar{\omega}-|\Delta \omega|\left(\cos \left(k_{x} a\right)+\cos \left(k_{y} a\right)+\cos \left(k_{z} a\right)\right) .
$$

For a frequency $\omega(\vec{k})=\omega_{0}=\bar{\omega}$ (middle of the band), the iso-frequency surface is the surface of a cube. In this case, propagation could only occur along the three space diagonal directions-regardless of the direction of the incident light! This phenomenon is referred to as self-collimation. It is directly connected to diffraction-less flow of light: a monochromatic Gaussian focus of light on the photonic-crystal surface would correspond to a Gaussian distribution of incident wave vectors of light. Via self-collimation, for an interface corresponding to the space diagonal, all of them would be translated into a single propagation direction, normal to the interface, inside the photonic crystal - for arbitrarily large distances and despite the fact that the incident lateral spatial profile is finite in extent. In vacuum, diffraction would obviously inhibit such behavior and rather lead to a finite opening angle [44]. 
a

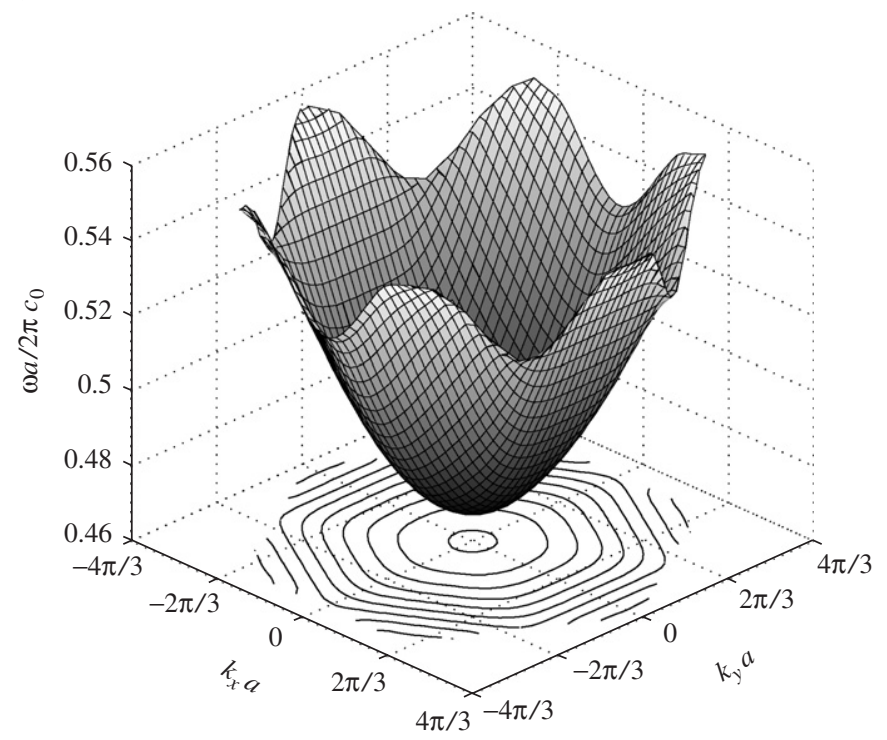

b

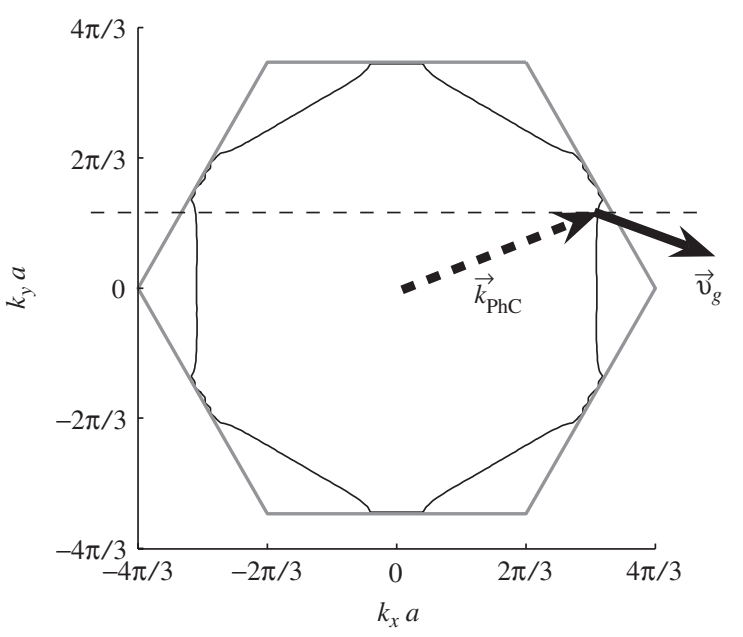

Fig. 7. Photonic bandstructure for $H$-polarized radiation propagating in hexagonal lattice of dielectric rods with radius $r=0.35 a$ and permittivity $\epsilon=12.96$ in air. The left and right panels depict the entire fourth band and the iso-frequency surface for a frequency $\omega a / 2 \pi c_{0}=0.535$, respectively. If a wave with this frequency and a parallel wave-vector component that is given by the dashed line is incident from the left onto a corresponding photonic-crystal interface, negative refraction occurs: The conservation of the parallel component of the wave vector together with the boundary condition that energy be transported away from the interface into the photonic crystal implies that the Bloch mode with wave vector $\vec{k}_{\mathrm{PhC}}$ is excited. As a result, the wave is refracted into the direction of this Bloch mode's group velocity $\vec{v}_{\mathrm{g}}$.

Another example is shown in Fig. 7 for a two-dimensional photonic crystal. Here, the incident flow of light is refracted at the photonic-crystal interface, exhibiting negative angles with respect to the surface normal inside the photonic crystal. In Snell's law, this would correspond to an effectively negative group index $n_{\text {group }}$. As the flow of light corresponds to the group velocity vector, this group index must not be confused with the usual refractive index, the phase index $n$, which is related to the wave vector of light and the phase velocity of light. Indeed, the photonic-crystal wave vector of light is refracted towards the usual side of the surface normal (Fig. 7). This aspect makes negative refraction in the context of photonic crystals distinct from negative refraction in metamaterials (see Section 5.4). There, the usual refractive index $n$, i.e., the phase index, will turn out to be negative under certain conditions. Unfortunately, both phenomena-though distinctly different in origin—are commonly referred to as "negative refraction" in the literature.

Finite-difference time-domain (see Section 2.1.4.3) calculations for finite-size photonic crystals reproduce the selfcollimation [45] and negative-refraction phenomena [46,47] that we have discussed on the basis of iso-frequency surfaces associated with the photonic-crystal band structure.

Historically, negative refraction has been known for a long time for interfaces of usual birefringent effective materials [48]. In 1968, Veselago [49] discussed negative refraction in the context of magnetic (meta)materials (see Section 5.4). For one-dimensional photonic crystals, Russell [50] in 1986 and Zengerle [51] in 1987 independently introduced the Bloch function picture that underlies our above reasoning for photonic crystals. In the long-wavelength limit of photonic crystals, negative refraction can simply arise due to form birefringence (see Section 2.1.1.1). In addition, self-collimation can occur for shorter wavelengths with respect to the lattice constant. Later, similar phenomena have been discussed for higher-dimensional photonic crystals [52-54]. Here, one has additional freedom in designing the band structure and, hence, the shape of the iso-frequency surfaces.

The shape of the iso-frequency surfaces sensitively depends on frequency. Thus, the direction of the flow of light inside the photonic crystal very sensitively depends on frequency for a fixed direction of the incident light. This effect can be used to construct so-called superprims [52], i.e., unusually dispersive prisms. Corresponding experiments will be discussed in Section 2.2.2. 


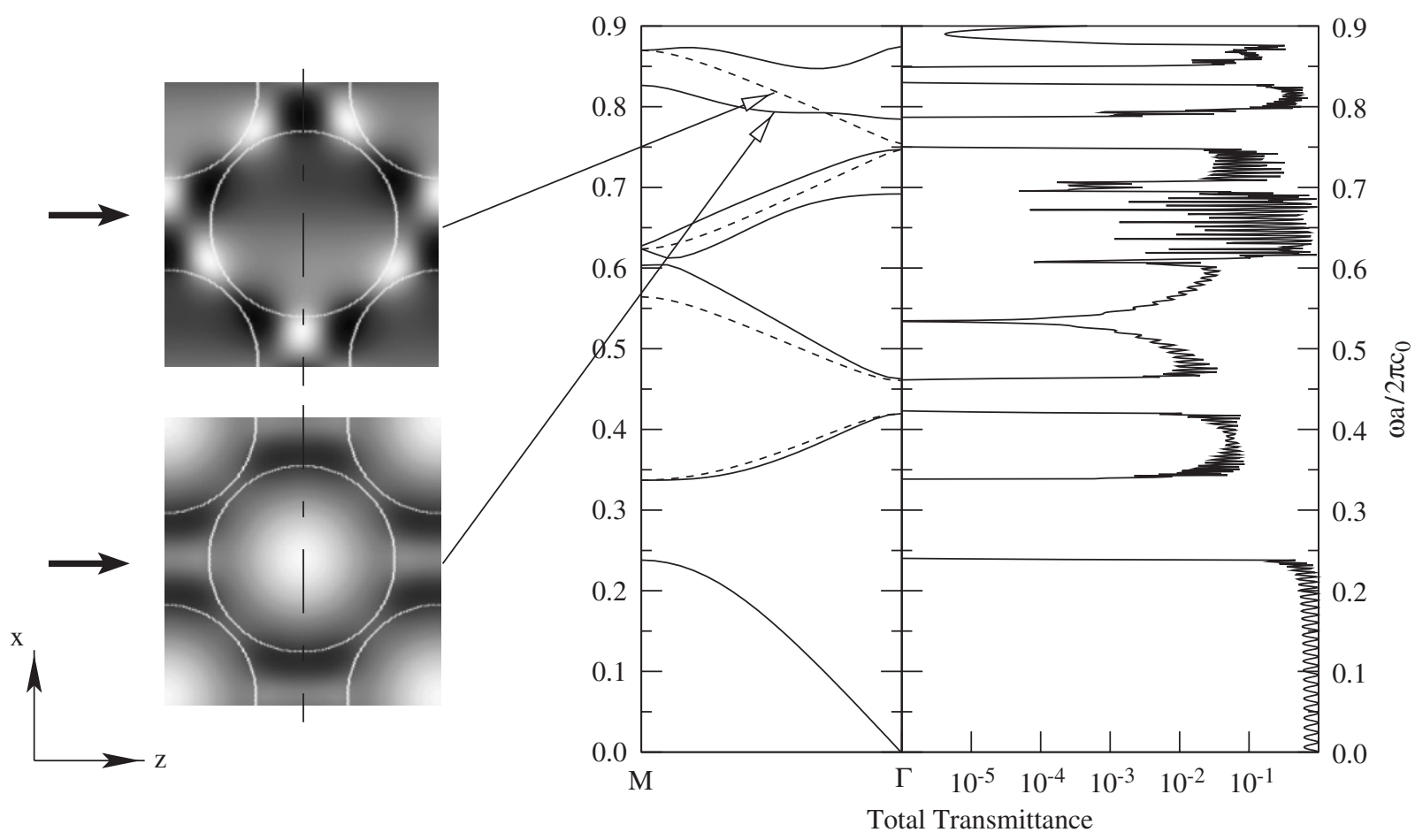

Fig. 8. Comparison between transmittance calculations (normal incidence) through a $2 \mathrm{D}$ photonic crystal oriented along the $\Gamma-M$ direction with the corresponding photonic band structure for the model system shown in Figs. 4 and 6. Uncoupled bands are marked by dashed lines. Bloch functions (electric field distributions) for a coupled and an uncoupled band are shown as gray-scale plots in the left panel. The position of the field maxima (white; corresponding to positive values) and minima (black; corresponding to negative values) reveal the odd parity of the uncoupled bands. The direction of the incoming radiation is indicated by black arrows and the surface termination of the photonic crystal is represented through the vertical dashed lines.

2.1.2.2. Uncoupled modes. In the reasoning of the previous paragraph, we have tacitly assumed that the light field impinging from vacuum or air can actually couple to the photonic-crystal mode at frequency $\omega(\vec{k})$ under consideration. However, higher-order Bloch modes may have a spatial symmetry (see, e.g., a mode depicted on the top left inset in Fig. 8) that forbids coupling to these modes. To illustrate this effect, we calculated normal-incidence transmittance spectrum of a 2D photonic crystal shown in Fig. 4. In Fig. 8, we compare these results with the corresponding band structure along the $\Gamma-M$ direction. The coupling strengths between plane and Bloch modes varies strongly, leading to corresponding variations in the total transmittance. The sharp resonances are associated with multiple reflections at the photonic-crystal front and end surfaces (Fabry-Perot effect) and are not of interest here. However, while the total transmittance for frequencies just above $\omega a / 2 \pi c_{0}=0.5$ is almost zero due to a very weak but nonvanishing coupling constant, there exists an extended frequency band just below $\omega a / 2 \pi c_{0}=0.8$, where the total transmittance vanishes exactly although the photonic-band structure comprises a band in this spectral regime. This seeming discrepancy is resolved when considering the symmetry of the corresponding Bloch functions: The Bloch functions for the band in question exhibit an odd parity across the slab surface. As the incoming plane wave has an even parity across the slab surface by construction, the coupling between the plane wave and the Bloch functions vanishes exactly due to the incompatible symmetry. The existence of such uncoupled bands can be inferred from a symmetry analysis $[9,55]$ of the photonic Bloch functions. In Fig. 8, all uncoupled bands for the $\Gamma-M$ direction are indicated by dashed curves.

Historically, uncoupled modes have first been discussed by Robertson in 1992 [56,57] and have later been further studied theoretically [58,59]. An analytical study of Bloch waves near photonic-crystal surfaces (specifically, the generalization of Heine's theory of metal/semiconductor interfaces) has also been carried out [60]. Obviously, the possibility of uncoupled bands should be accounted for when analyzing experimental transmittance data. 
2.1.2.3. Localized surface and interface modes. At the interface between different dispersive dielectric and/or magnetic materials, surface waves can occur [61]. We will briefly review this well-known fact in Example 4 in Section 5.4.1. In analogy, we may also expect particular surface modes at the interface of a photonic crystal and vacuum or air as well as at the interfaces between different photonic crystals. In view of the conceptual proximity to the physics of electronic semiconductor interfaces, systems containing one or several interfaces between different photonic crystals are often referred to as photonic-crystal heterostructures [62].

Surface modes may have a profound influence on the transmittance and reflectance characteristics of the corresponding interface and may be tailored via the termination of the photonic-crystal surfaces [63]. For instance, it has been found that suitably engineered surface modes of 2D photonic crystals allow for unusual focussing characteristics of light emerging from a W1 photonic-crystal waveguide [64] despite the fact that the photonic-crystal waveguide represents a sub-wavelength aperture (see Section 2.1.3.1 for more details on photonic-crystal waveguides and their nomenclature).

The actual calculation of localized surface modes utilizes the supercell methodology [65] (see Section 2.1.4.1), the scattering-matrix approach (see Section 2.1.4.2), or the related $R$-matrix approach [66]. In a photonic band structure, localized surface modes appear as solutions for frequencies in the photonic band gap of the corresponding infinite photonic crystal(s) and exhibit real-wave vectors parallel to the interface that lie outside the light cones of the materials/photonic crystal(s) to either side of the interface. Therefore, spectroscopy of these modes requires excitation via evanescent fields such as through prisms or through deviations in the periodicity along the interfaces. The latter is the case in the unusual focussing example alluded to above.

Historically, the existence of localized surface modes in the context of photonic crystals has first been established in 1991 by the Joannopoulos group via numerical calculations [65] and has been confirmed experimentally [67] in 1993. Since then, the study of localized surface modes in photonic crystals and, more generally, photonic-crystal heterostructures has received ever increasing attention and we refer to Ref. [62] for an up-to-date review of the corresponding research activities.

\subsubsection{Photonic-crystal cavities, waveguides, and functional elements}

In electronic micro-circuits, electrical currents are guided by thin metal wires in which electrons are bound within the cross section of the wire by the so-called work function (confining potential) of the metal. As a result, electrical currents follow prescribed pathways without escaping to the background. The situation for optical waves is rather different: Microscopic optical circuits are rather difficult to realize because empty space is already an ideal conductor of light waves. The light in traditional waveguiding structures based on total internal reflection can escape into the background electromagnetic modes of empty space if the structure is bent or distorted on a microscopic scale. PBGs in the band structure of photonic crystals remove this problem by removing all the background electromagnetic modes over the relevant band of frequencies. Consequently, light paths can be created inside a PBG material in the form of engineered waveguide channels — thus providing a promising avenue for highly integrated photonics.

2.1.3.1. Photonic-crystal cavities and waveguides. Creating a localized deviation from perfect periodicity destroys the translational symmetry of the ideal infinite photonic crystal. As a result, the Floquet-Bloch theorem (see Section 2.1.1.1) does not apply anymore and for photonic crystals that exhibit photonic band gaps a novel class of localized eigenmodes with eigenfrequencies inside the photonic band gap may develop. Depending on whether this deviation from periodicity results in an increase or decrease of the local dielectric function, these localized cavity modes develop near the upper or lower band edge, respectively. This behavior is quite analogous to the "doping" and the formation of donor and acceptor states of ordinary (electronic) crystals [8]. This is illustrated in Fig. 9 for the case of $H$-polarized radiation in a $2 \mathrm{D}$ photonic crystal where cavity modes are created by increasing the value of the dielectric function $\epsilon_{\mathrm{def}}$ of a single pore relative to the periodic case $\left(\epsilon_{\mathrm{def}}=1\right.$ is the periodic case). This corresponds to increasing the local dielectric constant and, therefore, leads to the formation of donor-like eigenmodes inside the photonic band gap of the underlying photonic crystal. The stronger the deviation from periodicity, the further these cavity modes are pushed into the photonic band gap and several distinct modes with different symmetry properties appear. Combining several of these simple cavities into more complex cavity structures allows a rather flexible engineering of their eigenfrequencies and mode profiles via considerations that are perfectly analogous to the method of linear combination of atomic orbitals (LCAO) $[8,40]$ where the individual modes of the simple cavity accept the role of the "atomic orbitals". Note that for low-to-moderate values of $\epsilon_{\text {def }}$ that correspond to typical liquid crystalline materials or polymers, this system exhibits only doubly degenerate dipole-like cavity modes. 


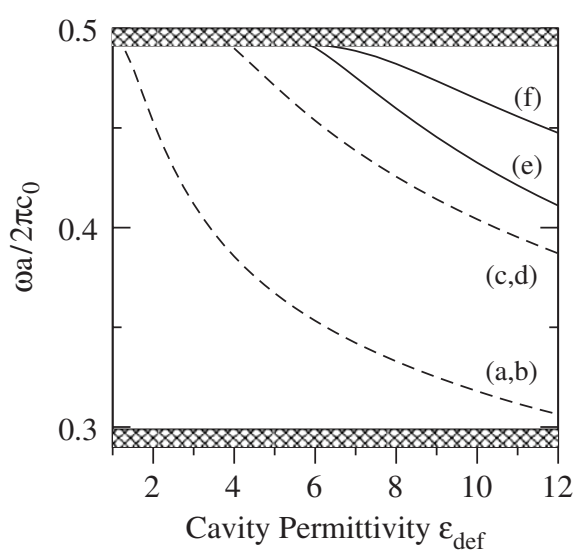

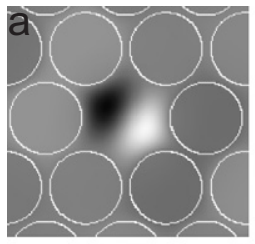
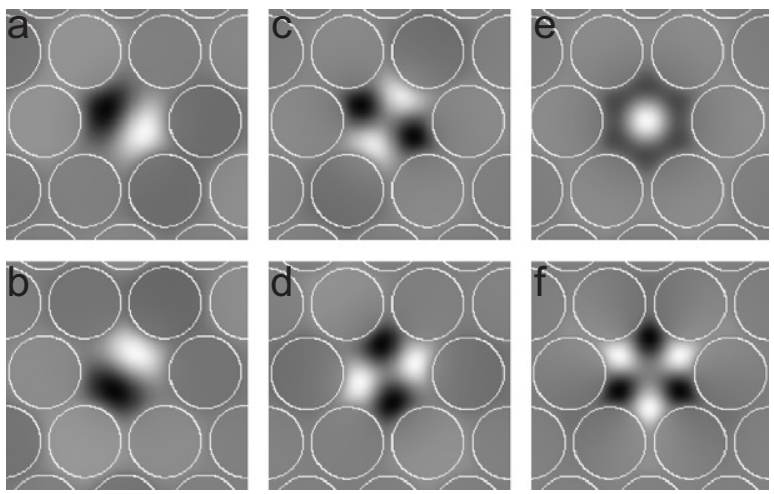

Fig. 9. Eigenfrequencies (left panel) and field distributions (right panel) of localized cavity modes for $H$-polarized light in a 2D photonic crystal composed of a hexagonal lattice of air pores (radius $r / a=0.45 a)$ in a dielectric matrix $(\epsilon=12)$ where the dielectric function of a single pore has been modified. The field distributions display the magnetic field on a gray color scale where white corresponds to positive and black to negative values. Depending on the strength $\epsilon_{\text {def }}$ of this deviation, different types of cavity modes such as doubly degenerate dipoles, (a) and (b), and quadrupoles, (c) and (d), as well as nondegenerate second-order monopoles, (e), and hexapoles, (f), develop. All results have been calculated by a plane-wave-based supercell approach (see Section 2.1.4.1).
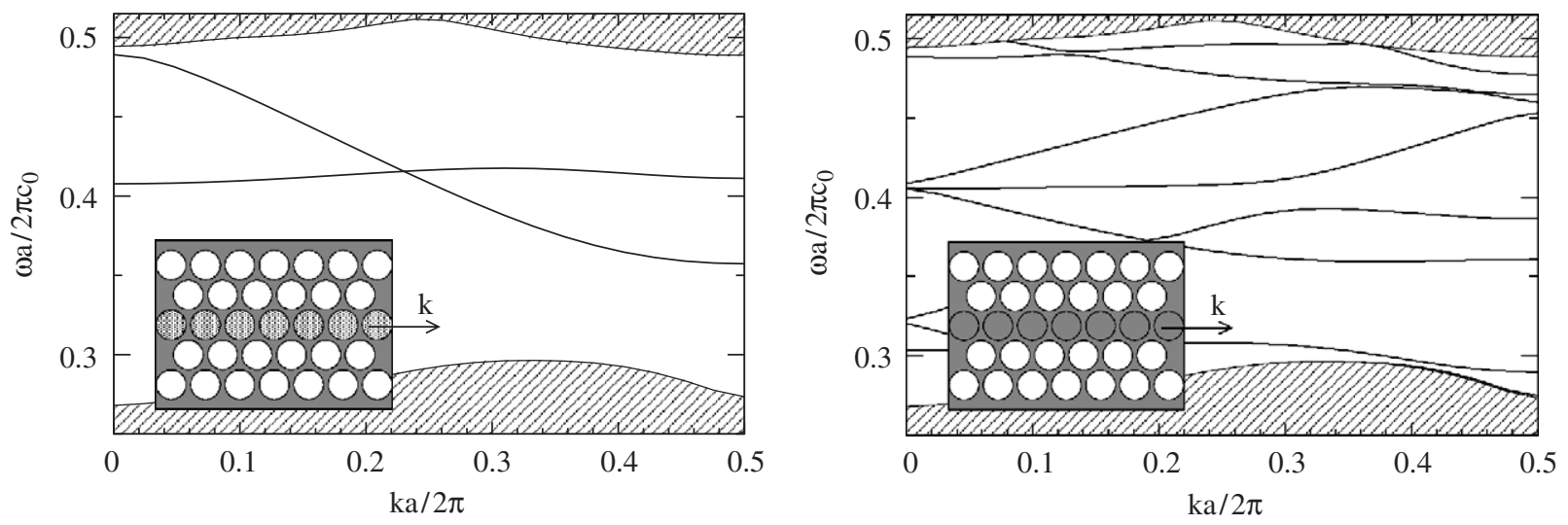

Fig. 10. Dispersion relation of W1 waveguides for $H$-polarized light in a 2D photonic crystal composed of a hexagonal lattice of air pores (radius $r / a=0.45 a)$ in a dielectric matrix $(\epsilon=12)$ where the dielectric function of a row of pores has been modified. Depending on the strength $\epsilon_{\mathrm{def}}$ of this modification, different regimes of waveguiding characteristics may be realized. For low values of the dielectric function $\left(\epsilon_{\mathrm{def}}=2.89\right.$; left panel), broad-band mono-mode waveguiding behavior occurs, whereas for high values of the dieletric function $\left(\epsilon_{\mathrm{def}}=12\right.$, corresponding to nonetched pores; right panel) complex multi-mode behavior is observed. All results have been calculated by a plane-wave-based supercell approach (see Section 2.1.4.1).

Chaining together an infinite number of such cavities into a one-dimensional line structure introduces a discrete translational symmetry into the corresponding spatial direction (also see CROW structures in Section 2.2.1). As a result, the Floquet-Bloch theorem (see Section 2.1.1.1) applies and leads to a dispersion relation for waveguiding modes with frequencies inside the photonic band gap of the underlying photonic crystal that are guided along this line of cavities. Based on the number $m$ of (parallel) rows of cavities that form the corresponding photoniccrystal waveguide, one classifies them as $\mathrm{W} m$-waveguides. Depending on this number $m$ and the number of cavity modes supported by an individual cavities, the resulting waveguide dispersion relations may exhibit a rather complex band structure. Mono- and multi-mode behavior, extremely low group velocities and very large group velocity dispersion may be realized. As an example, we display in Fig. 10 the dispersion relation of W1-photonic-crystal waveguides that are formed from single rows of low- and high-index cavities, respectively. The rather simple modal 

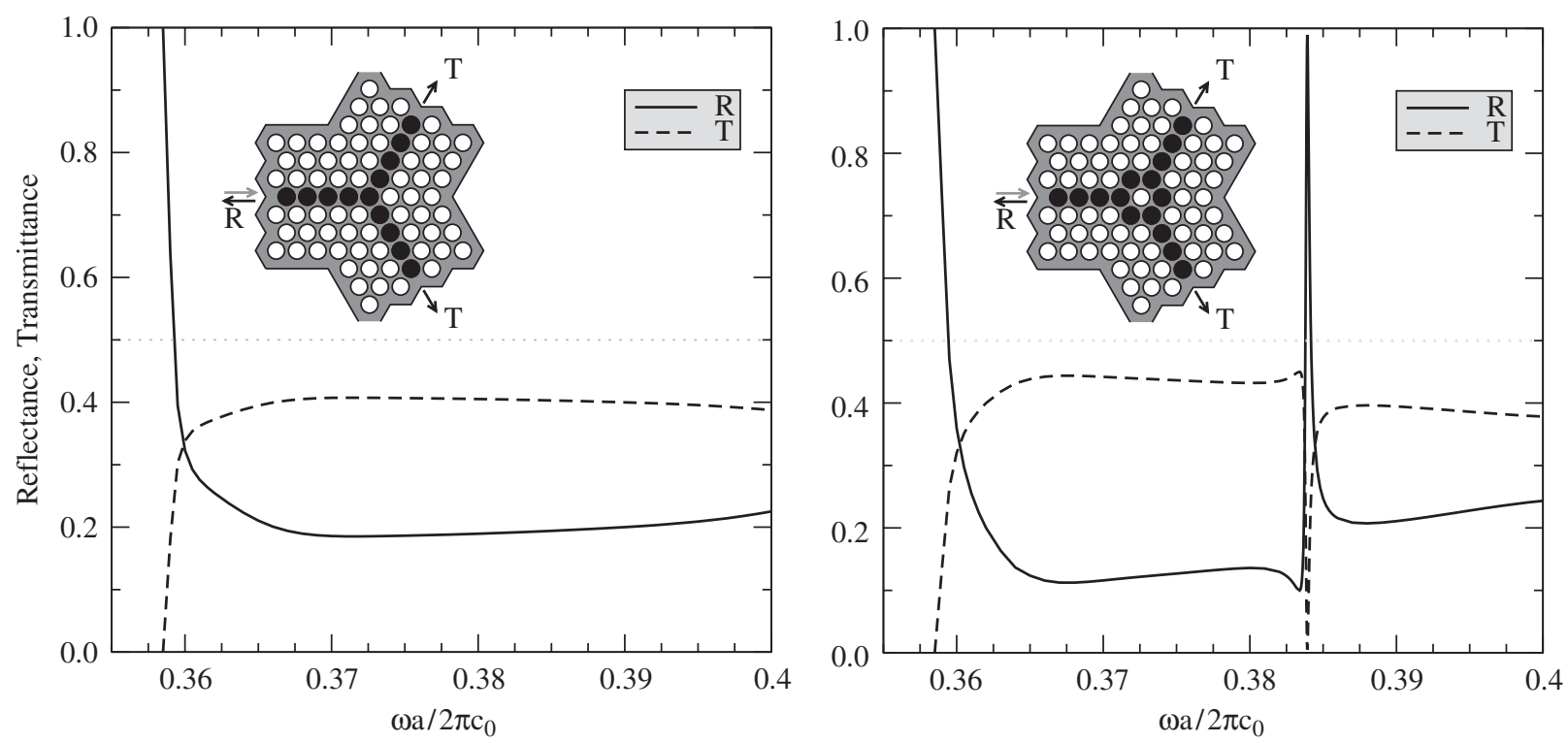

Fig. 11. Beamsplitter designs and their performance for the broad-band mono-mode W1 waveguides described in the left panel of Fig. 10. These results have been calculated by a Wannier-function approach (see Section 2.1.4.6). Reprinted figure with permission of D. Hermann and M. Schillinger.

structure of the corresponding individual cavity leads to broad-band mono-moded regions in the dispersion relation of the low-index W1-waveguide. Conversely, the complex behavior of the high-index individual cavities leads to a rich band structure of the corresponding W1-waveguiding modes where several regions that exhibit very low group velocities, i.e., so-called slow-light regimes, as well as regions with strong group velocity dispersion may be identified. Depending on the desired application and the experimental feasability one design may be preferred over the other.

2.1.3.2. Functional elements for photonic-crystal circuits. Photonic-crystal cavities and waveguides represent the key ingredients for the realization of more complex functional elements that eventually may lead to entire photonic-crystal circuits. More precisely, photonic-crystal waveguiding structures offer a number of attractive features that may fall into the categories of compactness and/or mode engineering. Strong interactions between light and matter in high-finesse cavities (see also Section 2.2.2) may allow for optical components such as sensors, logic gates, etc. that are orders of magnitude smaller than currently available devices. The tailoring of group velocities and group velocity dispersion allows to optimize chromatic dispersion and, thus, for novel pathways to advanced signal processing. Any of the aforementioned features can be enhanced through improved tunability that is realized by concentrating the optical field in regions that are infiltrated with tuning agents. These principles are illustrated in Fig. 11 where we depict two designs for beam splitters in 2D photonic crystals along with their performance. These designs utilize the broad-band mono-mode waveguides of Fig. 10 as input and output ports. Through a judicious design of the cavity that couples the various waveguides, it has been possible to improve the performance of the naive design (left panel of Fig. 11) over a rather large frequency range (see right panel of Fig. 11). For telecommunication applications at wavelengths of $1.55 \mu \mathrm{m}$, the resulting compact beam splitter occupies an area of about $4 \mu \mathrm{m}^{2}$.

Following the suggestion for sharp photonic-crystal waveguide bends [68] and add-drop filters [69], there have been numerous suggestions and designs for other and more complex functional elements based on photonic-crystal waveguiding structures and we refer to the recent literature for details $[13,70]$.

\subsubsection{Numerical methods}

There is no single numerical method capable of solving all problems related to periodic nanostructures for photonics. Instead, for each problem, a suitable approach has to be selected carefully from a plethora of methods, the most frequently used of which are outlined in the following seven paragraphs. 
2.1.4.1. Plane-wave method. A straightforward way of solving for eigenvalues and eigenfunctions of (2.10) and (2.11) for a strictly periodic system is to expand the dielectric function as well as the periodic part of the Bloch function into a Fourier series in reciprocal lattice vectors $[7,71,72]$. Owing to its simplicity and flexibility in handling practically any geometry of the unit cell, this so-called plane-wave method (PWM) has become the "work horse" for most investigations of photonic band structures. In practice, it turns out that it is more convenient to solve the wave equation for the magnetic field $\vec{H}(\vec{r}),(2.10)$ as the divergence-free nature of the magnetic field allows us to restrict the number of expansion coefficients to only two transverse polarizations per plane wave. However, there are two rather distinct strategies of solving (2.10) using plane waves.

The first approach consists of inserting the plane-wave expansions for the spatially periodic inverse dielectric function and the magnetic field

$$
\frac{1}{\epsilon(\vec{r})}=\sum_{\vec{G}} \eta_{\vec{G}} \mathrm{e}^{\mathrm{i} \overrightarrow{\vec{G}} \cdot \vec{r}} \quad \text { and } \quad \vec{H}_{\vec{k}}(\vec{r})=\sum_{\vec{G}} \sum_{\alpha=1}^{2} h_{\vec{G}}^{(\alpha)} \hat{e}_{\vec{G}}^{(\alpha)} \mathrm{e}^{\mathrm{i} \vec{G} \cdot \vec{r}}
$$

into the wave equation (2.10), thus transforming this equation into an eigenvalue problem for an infinite matrix which must be suitably truncated to become accessible to an approximate numerical solution

$$
\sum_{\vec{G}^{\prime}}|\vec{k}+\vec{G}|\left|\vec{k}+\vec{G}^{\prime}\right| \eta_{\vec{G}-\vec{G}^{\prime}}\left(\begin{array}{cc}
\hat{e}_{\vec{G}}^{2} \cdot \hat{e}_{\vec{G}^{\prime}}^{2} & -\hat{e}_{\vec{G}}^{2} \cdot \hat{e}_{\vec{G}^{\prime}}^{1} \\
-\hat{e}_{\vec{G}}^{1} \cdot \hat{e}_{\vec{G}^{\prime}}^{2} & \hat{e}_{\vec{G}}^{1} \cdot \hat{e}_{\vec{G}^{\prime}}^{1}
\end{array}\right)\left(\begin{array}{c}
h_{\vec{G}^{\prime}}^{1} \\
h_{\vec{G}^{\prime}}^{2}
\end{array}\right)=\frac{\omega_{\vec{k}}^{2}}{c_{0}^{2}}\left(\begin{array}{c}
h_{\vec{G}}^{1} \\
h_{\vec{G}}^{2}
\end{array}\right) .
$$

In (2.25) and (2.26), the polarization vectors can be chosen such that, together with the corresponding plane-wave vector $\vec{k}+\vec{G}$, they form an orthonormal triad

$$
|\vec{k}+\vec{G}|\left(\hat{e}_{\vec{G}}^{(1)} \times \hat{e}_{\vec{G}}^{(2)}\right)=\vec{k}+\vec{G} .
$$

As a result, for a total number of $N$ plane waves, this leads to a full $2 N \times 2 N$ matrix problem. In general, the Toeplitz matrix of Fourier coefficients of the inverse dielectric function $\eta_{\vec{G}, \vec{G}^{\prime}} \equiv \eta_{\vec{G}-\vec{G}^{\prime}}$ with

$$
\eta_{\vec{G}}=\frac{1}{V_{\mathrm{WSC}}} \int_{\mathrm{WSC}} \mathrm{d}^{3} r \frac{1}{\epsilon(\vec{r})} \mathrm{e}^{-\mathrm{i} \vec{G} \cdot \vec{r}}
$$

must be computed numerically via fast Fourier transform (FFT). The integration extends over the Wigner-Seitz cell (WSC) with volume $V_{\text {WSC }}$. In practice, this Toeplitz matrix can be obtained in two ways: One can calculate the inverse dielectric function in real space and then compute its Fourier coefficients (direct method). Alternatively, one can calculate the matrix of Fourier coefficients of the dielectric function and then take its inverse to obtain the required Fourier coefficients (Ho-Chan-Soukoulis (HCS) method [7]). Since the operations of taking the Fourier transform and inversion commute on the complete (and infinite) set of plane waves, the results for the direct method and for the HCS method must coincide exactly. However, numerically we are restricted to operate on a finite-dimensional subspace of the full reciprocal space. This leads to significantly different rates of convergence of the two methods as the subspace (number of plane waves) is increased. Detailed investigations [32,73] have demonstrated the excellent convergence properties of the HCS method.

The second strategy starts from the observation that in the wave equation (2.10), the curl operator is diagonal in reciprocal space while the multiplication with the inverse dielectric function corresponds to a diagonal operation in real space. This suggests that an efficient way of solving (2.10) consists of an iterative approach for both, eigenfunctions and eigenvalues, where only diagonal operators are employed and the (required) transitions between real and reciprocal space are carried out with highly efficient FFT-algorithms [74,75]. As a result, the number of plane waves will be as large as the number of real-space grid points that are used to sample the unit cell. Together with the sparsity of the diagonal operators, this method can utilize many more plane waves than the standard PWM. This has led to the claim that the iterative PWM exhibits significantly better convergence rates for the experimentally most relevant case of structures with step discontinuities in the dielectric function [74].

However, the eigenfrequencies of the lowest lying bands are determined through the interaction of relatively few Bragg orders, so that the rate of convergence of PWM calculations depends rather strongly on the accurate representation of the corresponding Fourier coefficients. Consequently, standard PWM as well as iterative PWM are subject to the 
Fourier sampling theorems which require a sufficient over-sampling of the unit cell. Since within iterative PWM the number of plane waves is determined by the real-space discretization, the Fourier sampling theorems enforce the use of relatively large numbers of plane waves in order to obtain converged results. In standard PWM, the number of plane waves can be chosen independently from the real-space grid employed for obtaining the Fourier coefficients via FFT. In fact, standard PWM can also accommodate analytically available Fourier coefficients, such as those for nonoverlapping cylinders or spheres. Once the Fourier coefficients are determined accurately, the convergence of standard PWM depends on the number of plane waves employed and has to be carefully checked. For typical high-index structures such as 3D silicon photonic crystals, one finds that standard PWM equipped with the HCS-method requires about $N_{\mathrm{st}}=2^{10}=1024$ plane waves in order to achieve the same accuracy as iterative PWM obtains when using $N_{\mathrm{it}}=2^{16}=65$, 536 plane waves. In addition, the computational resources of both PWM approaches are very similar with home-made standard PWM [32] having a small edge over the freely available iterative PWM (http://ab-initio.mit.edu/mpb/). However, we want to note that further improvements of the PWM methodology will most likely originate from the use of adaptive coordinates [76].

Using an artificially enlarged unit cell, the PWM may also be employed to calculate the localized eigenmodes of simple cavities and the dispersion relations of simple waveguides embedded in photonic-band-gap materials. In this so-called supercell technique [8,9], the unit cell has to be chosen sufficiently large so that the actual unit cell boundary conditions become irrelevant. Finally, we want to mention that the PWM can also be implemented for the electric field $\vec{E}$ as well as for the displacement field $\vec{D}$ [71,72]. Moreover, it is also possible to carry out on-shell calculations [77], i.e., find the allowed wave vectors for a given frequency —instead of the reverse situation discussed above. This is the key ingredient for the so-called scattering-matrix technique (see Section 2.1.4.2).

2.1.4.2. Scattering-matrix technique. Transmittance and reflectance spectroscopy is the most popular method for the characterization of photonic-crystal structures cut along certain lattice planes. From a theoretical point of view, the resulting problem bears a close resemblance to certain problems in diffractive optics: A plane wave of given frequency impinges onto a laterally infinitely extended and periodically structured material with a finite extent along the propagation direction. In the case of photonic-crystal structures, the "gratings" become very deep, i.e., typically several tens of wavelengths and the unit cell geometry can be rather complex. In addition, the full vectorial nature of the electromagnetic field has to be considered.

A rather popular strategy for solving this problem consists in a two-stage approach. First, the structure is discretized along the propagation direction in a stair-case fashion ("slicing"). Owing to the homogeneity in propagation direction, one may decompose the electromagnetic field in each slice into a set of eigenmodes with unknown propagation constants (i.e., the wave-vector components along the propagation direction). These modes, together with their propagation constants, may be determined by noting that for a given frequency and direction of the incoming plane wave, the lateral wave vector is conserved so that an on-shell plane wave band structure calculation (see Section 2.1.4.1) becomes possible. Similar to the case of plane-wave-based band structure calculations, proper Fourier factorization [78] leads to considerable convergence speed-ups. In a second step, the expansions of the electromagnetic field in different slices have to be made consistent by enforcing appropriate boundary conditions between slices. Thus, two adjacent slices may be combined to one larger slice. Here, care has to be taken in order to avoid exponentially growing factors that originate from the evanescent modes in the slices. This can be accomplished in the framework of one of several recursive scattering-matrix algorithms [79] that relate the outgoing modes from each slice with the corresponding incoming modes onto that slice. When all internal boundaries between slices have been eliminated, the total scattering matrix of the entire photonic-crystal structure is obtained. From this scattering matrix, one may read off the transmittance coefficients into the Bragg orders on the far side of the photonic crystal (as seen from the incoming plane wave) as well as the reflectance coefficients into the reflected Bragg orders.

In the diffractive optics community, the approach described above is generally referred to as rigorous coupled wave analysis (or Fourier modal method) [80-83] while in the photonic-crystal community it is more often known as scattering-matrix approach $[84,85]$. It has, for instance, been employed to characterize the angle-resolved reflectance and transmittance spectra of 2D [86] and 3D photonic crystals [87,88] (see also Section 2.2.3.1) as well as 2D photoniccrystal slabs [89]. Detailed comparison between experimental data and numerical calculations can provide information about the optical quality of photonic-crystal samples that is hard to come by otherwise [90]. Finally, we want to note that when equipped with appropriate absorbing boundary conditions, the scattering-matrix approach can be extended to nonperiodic systems [91,92]. 
2.1.4.3. Finite-difference time-domain method. Photonic crystals and metamaterials represent very prominent subclasses of nano-photonic systems that are characterized through complex geometries and/or complex material properties such as optical anisotropies and nonlinearities. As a result, fully vectorial approaches to solving Maxwell's equations play a prominent role in developing a quantitative understanding, both descriptive and predictive, of these systems. Among the various established methods, the finite-difference time-domain (FDTD) $[93,94]$ technique is the most popular approach and several sophisticated commercial software packages are available (e.g., the RSOFT FullWAVE and the CST MICROWAVE STUDIO design tools).

In its simplest form [93], the FDTD approach discretizes space and time on an equidistant lattice in such a way that all derivatives that occur in Maxwell's equations are replaced by central differences. The mathematical structure of the two curl equations suggests that a staggered real-space grid together with a leapfrog scheme in time should be used in order to efficiently implement Ampère's law and Faraday's law. As a result, the electric and magnetic fields are defined at different real-space points and for different times, so that considerable care has to be exerted when defining derived quantities such as the Poynting vector and the phase of reflected/transmitted fields [94]. The resulting finite-difference equations are very intuitive and may be rearranged into a form that is straightforward to implement and ideally suited for modern CPU architectures. Today, even special FDTD software is available that exploits hardware acceleration of reprogrammable FPGA boards.

The nature of most physical problems that will be addressed with any general purpose real-space and real-time algorithm such as the FDTD method requires a careful treatment of open (transparent) boundary conditions for the termination of the computational domain. Furthermore, internal dielectric/metallic boundaries have to be discretized appropriately. To date, the so-called perfectly matched layers (PMLs) [95] that come in various forms [94] are sufficient for most applications. For more specific applications such as photonic-crystal waveguides where high accuracy is required, advanced boundary conditions have been developed [96]. In contrast to the rather mature state of advanced open boundary conditions for FDTD, the treatment of arbitrarily shaped internal boundaries represents a rather contentious problem. Here, one may employ simple staircasing, finite-volume discretizations, nonuniform grids, and contour-path discretizations from the integral form of Maxwell's equation. For a recent overview, we refer to Refs. [97,94] and references therein.

The stability of the basic FDTD approach may be characterized by the Courant-number

$$
C=\max _{m}\left\{\frac{\Delta t}{\Delta x} \frac{c_{0}}{n_{m}}\right\}
$$

where $\Delta x$ and $\Delta t$, respectively, denote the real-space and time discretization and the set $\left\{n_{m}, m=1,2, \ldots\right\}$ comprises the refractive indices of the different materials that occur in the computational domain. The FDTD-approach becomes numerically unstable for $C>1$ and exhibits considerable numerical dispersion for $C$ near unity [94]. This intrinsic limitation of the basic FDTD method requires the use of formidable computational resources when attempting accurate long-time simulations for frequencies near photonic band edges. The same applies for numerically large systems where, for instance, the computational domain consists of many unit cells of a photonic crystal or metamaterial. This situation has initiated considerable research regarding improved FDTD methods, for instance by using higher-order discretizations [98] or wavelet collocation schemes [99]. In fact, the stability problem of the basic FDTD algorithm can be removed using an implicit difference scheme known as alternating-direction-implicit (ADI) FDTD [100]. Some of these improved approaches also reduce the problem of numerical dispersion [101]. However, it has to be emphasized that all of these approaches also lead to considerable computational overhead relative to the basic FDTD algorithm.

Therefore, recent years have seen considerable efforts to develop alternative time-domain simulation techniques based on finite-element discretizations [102] (see following section) and operator-exponential techniques [103,104]. Unfortunately, the finite-element time-domain technique (FETD) [102] requires at every time step to solve a large (but sparse) system of equations. This leads to significantly more CPU operations per time step and, therefore, largely off-sets the advantages obtained by an adaptive real-space discretization. The operator-exponential technique based on the Lie-Suzuki-Trotter formula [103] results in an unconditionally stable algorithm with low dispersion but cannot include PMLs in more than one spatial direction. Similarly, operator-exponential methods that rely on Chebyshevpolynomial expansions [104] provide very high accuracy for extremely long-time simulations but are only conditionally stable and strictly limited to hard-wall boundary conditions and nonabsorbing materials. A recent modification of this method utilizes a Faber-polynomial expansion technique [105]. Although this modification allows to include losses, the implementation of PMLs still remains an open issue. While these methods provide very interesting features, to date they 
have been unable to demonstrate a significantly better performance than standard FDTD for real-space and real-time simulations of complex photonic structures, including photonic crystals and metamaterials. Recent developments on Krylov-subspace methods could offer a promising new route [106].

2.1.4.4. Finite-element approaches. The finite-element method (FEM) constitutes another general-purpose approach for complex optical systems. In order to solve the wave equation for either the magnetic or the electric field in the frequency domain, (2.10) or (2.11), together with appropriate boundary conditions, standard FEM proceeds in three steps [102]. First, (2.10) or (2.11) are identified as solutions of certain variational problems where the boundary conditions at the surface $\partial V$ of the computational domain $V$ have been incorporated as additional terms in the Lagrangian $\mathscr{L}$. For the electric field, the most general variational formulation reads

$$
\begin{aligned}
\mathscr{L}(\vec{E})= & \frac{1}{2} \int_{V} \mathrm{~d}^{3} r\left[\frac{1}{\mu}(\nabla \times \vec{E}) \cdot(\nabla \times \vec{E})-\frac{\omega^{2}}{c_{0}^{2}} \epsilon \vec{E} \cdot \vec{E}\right]+\int_{\partial V} \mathrm{~d} \vec{S} \cdot\left[\frac{\gamma_{e}}{2}(\hat{n} \times \vec{E}) \cdot(\hat{n} \times \vec{E})+\vec{E} \cdot \vec{U}\right] \\
& +\mathrm{i} \frac{\omega}{c_{0}} \sqrt{\frac{\mu_{0}}{\epsilon_{0}}} \int_{V} \mathrm{~d}^{3} r \vec{E} \cdot \vec{j} .
\end{aligned}
$$

Here, both the magnetic permeability $\mu$ and the dielectric function $\epsilon$ may vary in space. In addition, $\hat{n}$ denotes the outward normal at the surface $\partial V$ and the electric field has to satisfy the Dirichlet boundary condition $\hat{n} \times \vec{E}=0$ on $\partial S$. $\gamma_{e}$ and $\vec{U}$ are known quantities which are used to represent various other types of boundary conditions such as impedance boundary conditions and Sommerfeld radiation conditions (also known as outgoing wave boundary conditions) [102]. Finally, radiation sources within the computational domain $V$ are described through the spatially varying current density $\vec{j}$.

The second and most demanding step consists of the discretization of the Lagrangian. To this end, the computational domain $V$ is subdivided into a number of small-volume elements, the so-called finite elements. These finite elements, e.g., in three dimensions of tetrahedral shape, are chosen such that all internal boundaries (surfaces) between materials are approximated by triangular elements. Within each element, the electric field is expanded into a series of certain elementary functions with unknown coefficients. In particular, using the so-called Whitney or vector elements [102], it becomes possible to approximately enforce the div-conditions of the electric field within a given element as long as the dielectric function does not vary within this element.

In the final step, these expansions facilitate the transformation of the variational equation (2.30) into a sparse set of linear equations via the Galerkin method. Here, care has to be taken when assembling the matrices corresponding to the different terms in (2.30): Local expansions in the individual elements have to be made globally consistent through the boundary conditions between them and an appropriate global numbering that generates well-behaved matrices has to be introduced. This matrix system can subsequently be solved via advanced linear algebra methods, either for obtaining eigenfrequencies and eigenmodes of the system of interest or to determine scattering cross sections of complex structures as well as transmittance and reflectance through functional elements.

The flexibility of the adaptive discretization together with the usage of vector elements represents a highly desirable feature of FEM. The former allows to accurately represent arbitrary material interfaces and reduces the use of computational resources. The latter avoids spurious influences from non-Maxwellian fields. These features, however, have their price. FEM codes are typically very involved and difficult to implement. In particular, the construction of higherorder elementary functions and the problem of adaptive refinement of a given mesh (discretization) are still subject of active research in applied mathematics. Several commercially available FEM packages (e.g., COMSOL multiphysics (formerly FEMLab) or JCM Wave) offer all-purpose capabilities for complex optical structures. Nevertheless, the full power of FEM has been utilized for complete three-dimensional photonic bandstructure calculations as well as transmittance/reflectance calculations only recently [107]. In addition, the highly accurate modeling of arbitrary material interfaces has stimulated the adaptation of the well-known topology-optimization technique from structural engineering to the design of photonic-crystal functional elements in two-dimensional systems. The realization of several designs for two-dimensional photonic-crystal slab structures highlights their quite remarkable performance $[108,109]$. It remains to be seen whether easy-to-fabricate designs can be developed for incorporation into more complex photonic-crystal circuitry.

As alluded to above, the finite-element discretization scheme can also be applied to time-dependent problems [102]. Unfortunately, all FETD formulations known to date suffer from the fact that at every time step a matrix inversion 
has to be carried out which largely off-sets the advantages in CPU-resource usage gained from the finite-element discretization. Nevertheless, recent progress in the FETD of periodic systems [110] and the development of advanced elementary functions for the use with PMLs [111] demonstrate the excellent stability properties of FETD.

2.1.4.5. Multiple-multipole method. Another approach to solving (2.11) is to adapt the method of Kohn, Korringa and Rostocker (KKR) familiar from electronic band structure theory [8] to the case of periodic dielectric structures. Within the KKR method, the fields are expanded into series of (vector) spherical harmonic functions centered around individual lattice sites. For uniform and cylindrically or radially symmetric scatterers, the corresponding radial functions become Bessel functions and Graf's addition theorem then allows to relate the electromagnetic field resulting from the radiation that emanates from all but the central scatterer to the electromagnetic field expanded around this central scatterer. In electronic structure theory, the KKR method has subsequently been extended to nonspherical scattering potentials. In electromagnetic theory, a closely related method based on arbitrary basis functions and scatterers has been developed and is generally known as the multiple-multipole (MMP) method [112]. For strictly periodic systems of spherical scatterers, the two methods become identical and map (2.11) onto an eigenvalue problem of an infinite matrix system. This statement holds for both, two-dimensional [113,114] and three-dimensional systems [115-117]. As a result, electromagnetic boundary conditions associated with discontinuities of the dielectric function of spherically symmetric scatterers may be represented to arbitrary accuracy. In fact, for these systems, the KKR method proves to be more efficient than PWM, allowing to check the performance of PWM band structure calculations [116].

For spherically symmetric systems, KKR and PWM may be regarded as complementary: KKR relies on the eigenfunctions of the individual scatterer and, therefore, allows to represent the discontinuities of the dielectric function very accurately while the lattice structure is incorporated via the multi-center expansion technique encoded into Graf's addition theorem. Conversely, PWM is based on an accurate representation of the underlying lattice structure via the expansion into a set of plane waves that obey the Floquet-Bloch theorem and incorporates discontinuities in the dielectric function through the corresponding Fourier coefficients. However, for nonspherical scatterers, the KKR method loses its most attractive feature of accurately incorporating the discontinuities of the dielectric function. In view of the numerous structures with nonspherical scatterers, notably in three dimensions, the KKR method has, therefore, been unable to replace PWM as the "work horse" of photonic band structure calculations.

However, since the KKR-approach explicitly considers each scatterer as the source of secondary waves and solves the entire multiple scattering problem by relating local expansions around each scatterer to a global expansion around an arbitrary (user-defined) point in space, the method can be applied to a finite number of scatterers and may include multiple sources [38]. This allows to determine the local density of states (see Section 2.1.1.2) of finite clusters of scatterers, thus providing valuable information for the radiative dynamics of emitters embedded in photonic crystals and to characterize nonperiodic structures such as curvilinear photonic crystals [36] or photonic quasi-crystals for which dispersion relations and related concepts such as group velocity cannot easily be defined.

In addition, the KKR approach may be combined with transfer matrix and/or scattering-matrix methods (see Section 2.1.4.2) in order to facilitate transmittance and reflectance calculations through photonic-crystal functional elements and from slabs of ordered and disordered photonic crystals. The resulting efficient modeling and simulations tools allow to optimize designs [118,119], to characterize finite-size photonic-crystal samples $[120,121]$, and to investigate the effects of disorder on wave propagation in photonic crystals [122,123].

2.1.4.6. Wannier-function approach. A natural description of localized cavity modes and waveguiding structures in photonic-band-gap materials consists of an expansion of the electromagnetic field into a complete set of localized basis functions which have encoded into them all the information of the underlying photonic band structure. Since the latter is readily available from the complete set of extended photonic Bloch functions (see Section 2.1.1.1), one may employ the vast knowledge of electronic structure theory $[8,124,125]$ and define the so-called photonic Wannier functions

$$
\vec{W}_{n \vec{R}}(\vec{r})=\frac{V_{\mathrm{WSC}}}{(2 \pi)^{d}} \int_{\mathrm{BZ}} \mathrm{d}^{d} k \mathrm{e}^{-\mathrm{i} \vec{k} \vec{R}} \vec{E}_{n \vec{k}}(\vec{r})
$$

Here, $V_{\text {WSC }}$ denotes the volume of the Wigner-Seitz cell. The above definition-basically a lattice-Fourier transform of the Bloch functions-associates the photonic Wannier functions $\vec{W}_{n \vec{R}}(\vec{r})$ with the frequency range covered by band $n$, and centers it around the corresponding lattice site $\vec{R}$. In addition, the completeness and orthogonality as well as the 
translational properties of the Bloch functions translate directly into corresponding properties of the photonic Wannier functions.

Computing the Wannier functions directly from the output of photonic band structure programs via (2.31) leads to functions with poor localization properties and erratic behavior (see, for instance, Fig. 2 in Ref. [126]). The problems originate from an indeterminacy of the global phases of the Bloch functions (see Section 2.1.1.1). It is straightforward to show that for a group of several bands (we define their number as $N_{\mathrm{w}}$ ) there exists, for every wave vector $\vec{k}$, a free unitary transformation $U_{m n}$ between the bands which leaves the orthogonality relation of Wannier functions (2.31) unchanged

$$
\vec{E}_{n \vec{k}}(\vec{r}) \rightarrow \sum_{n=1}^{N_{\mathrm{w}}} U_{m n}(\vec{k}) \vec{E}_{m \vec{k}}(\vec{r}) .
$$

Recent progress in electronic structure theory $[127,128]$ has shown that this "gauge freedom" may be utilized to obtain maximally localized Wannier functions by numerically determining a unitary transformation between the bands that minimizes an appropriate spread functional

$$
F\left[\left\{U_{m n}(\vec{k})\right\}\right]=\sum_{n=1}^{N_{\mathrm{W}}}\left[\left\langle n \overrightarrow{0}\left|r^{2}\right| n \overrightarrow{0}\right\rangle-(\langle n \overrightarrow{0}|\vec{r}| n \overrightarrow{0}\rangle)^{2}\right],
$$

where

$$
\left\langle n \vec{R}|\hat{f}| n^{\prime} \vec{R}^{\prime}\right\rangle=\int_{\text {all space }} \mathrm{d}^{d} r \epsilon_{\mathrm{p}}(\vec{r}) \vec{W}_{n \vec{R}}^{*}(\vec{r}) \hat{f} \vec{W}_{n^{\prime} \vec{R}^{\prime}}(\vec{r}),
$$

denotes a matrix element of an arbitrary operator $\hat{f}$ within the Wannier function representation (note the weighting factor $\epsilon_{\mathrm{p}}(\vec{r})$, i.e., the dielectric function of the strictly periodic photonic crystal). It should be pointed out that instead of working with the electric field $[126,129,130]$ one may equally well construct photonic Wannier functions for the magnetic field [131].

Once the photonic Wannier functions are computed, the electric field is expanded according to

$$
\vec{E}(\vec{r})=\sum_{n, \vec{R}} E_{n \vec{R}} \vec{W}_{n \vec{R}}(\vec{r})
$$

with unknown amplitudes $E_{n \vec{R}}$. Inserting this expansion into the wave equation (2.11) leads to sparse and-in comparison with other known techniques that rely on an expansion into basis functions such as finite element or wavelet techniques-low-rank systems of equations for the amplitudes $E_{n} \vec{R}$. This facilitates the efficient computation of cavity modes, waveguide dispersion relations, and transmittance/reflectance coefficients through complex functional elements embedded in photonic band gap materials. For a review of these techniques, we refer to Ref. [126]. In addition, the Wannier-function approach allows to formulate a photonic-crystal circuit theory [132] where functional elements are replaced by simple "impedance" or "Jones" matrices that may be combined to form more complex circuitry. This Wannier-function approach has been combined with low-rank update techniques [133] and sensitivity analyses [134] that allow the highly efficient optimization of photonic-crystal functional elements such as mode separators [133] and broad-band low-crosstalk waveguide intersections [135].

Historically, the idea that the Wannier-function approach should be very efficient when applied to photonic-crystal structures has first been suggested by Leung [136]. The first semi-quantitative Wannier-function calculations for E-polarized radiation in two-dimensional photonic crystals [137] have been based on a tight-binding formulation of the underlying photonic band structure [137,138]. Unfortunately, to date no successful tight-binding parametrization for the case of $H$-polarization in 2D photonic crystals or for 3D photonic crystals exists (see Ref. [126]). Finally, we want to mention that several authors have employed Wannier-like envelope equations to study pulse propagation in photonic crystals $[139,140]$.

2.1.4.7. Green's function technique. The Green's function approach constitutes a very powerful general-purpose approach for solving wave propagation and scattering problems in various disciplines in physics [141,142] (see also 
Chapter 7 in Ref. [143]). In the present case of a vectorial wave equation for the electric field (2.11), the Green's function $\mathbf{G}\left(\vec{r}, \vec{r}^{\prime} ; \omega\right)$ exhibits a tensor structure that represents the electric-field distribution generated by a point-like source located at $\vec{r}^{\prime}$ and radiating with frequency $\omega$

$$
\left(\nabla \times \nabla \times-\frac{\omega^{2}}{c_{0}^{2}} \epsilon(\vec{r})\right) \mathbf{G}\left(\vec{r}, \vec{r}^{\prime} ; \omega\right)=\mathbf{I} \delta\left(\vec{r}-\vec{r}^{\prime}\right) .
$$

Here, I denotes the unit tensor and the dielectric function $\epsilon(\vec{r})$ describes the—as yet arbitrary—distribution of dielectric material of the system.

If (2.36) can be solved—either analytically such as in free space [141] or numerically—for a reference system, i.e., for a given $\epsilon_{\text {ref }}(\vec{r})$, the electric field in (2.11) can be represented according to

$$
\vec{E}(\vec{r})=\vec{E}_{\mathrm{ref}}(\vec{r})+\frac{\omega^{2}}{c_{0}^{2}} \int \mathrm{d}^{3} r^{\prime} \mathbf{G}_{\mathrm{ref}}\left(\vec{r}, \vec{r}^{\prime} ; \omega\right) \Delta \epsilon\left(\vec{r}^{\prime}\right) \vec{E}\left(\vec{r}^{\prime}\right) .
$$

In this Lippmann-Schwinger equation (2.37), the reference Green's function $\mathbf{G}_{\mathrm{ref}}\left(\vec{r}, \vec{r}^{\prime} ; \omega\right)$ acts as an integral kernel on the right-hand side that describes the multiple scattering of the electric field brought about by the deviation from the reference system $\Delta \epsilon(\vec{r})=\epsilon(\vec{r})-\epsilon_{\text {ref }}(\vec{r})$. In addition, $\vec{E}_{\text {ref }}(\vec{r})$ represents a solution of the reference system's wave equation that facilitates the realization of appropriate boundary conditions.

For a strictly periodic photonic crystal, the corresponding Green's function $\mathbf{G}_{\mathrm{p}}\left(\vec{r}, \vec{r}^{\prime} ; \omega\right)$ may be expressed in terms of Bloch functions $\vec{E}_{n \vec{k}}(\vec{r})$ and the corresponding eigenfrequencies $\omega_{n \vec{k}}$ via

$$
\mathbf{G}_{\mathrm{p}}\left(\vec{r}, \vec{r}^{\prime} ; \omega\right)=\sum_{n} \int_{\mathrm{BZ}} \mathrm{d}^{3} k \frac{\vec{E}_{n \vec{k}}^{*}(\vec{r}) \otimes \vec{E}_{n \vec{k}}\left(\vec{r}^{\prime}\right)}{\omega_{n \vec{k}}^{2} / c_{0}^{2}-\left(\omega / c_{0}^{2}+\mathrm{i} 0_{+}\right)^{2}} .
$$

Here, $\otimes$ denotes the dyadic product of two vectors. It is important to note that the summation/integration in (2.38) has to include those Bloch functions which obey the wave equation (2.11) but which do not obey the divergence condition $\nabla \cdot \vec{D}(\vec{r})=0$. This is related to the fact that in three dimensions, the physical solutions of the wave equations (2.11) and (2.10) are no longer a complete basis set in the mathematical sense. However, in most cases of practical interest the problem can be reformulated to include the physical solutions only (see Refs. [9,43]). Consequently, $\mathbf{G}_{\mathrm{p}}\left(\vec{r}, \vec{r}^{\prime} ; \omega\right)$ may serve as a reference Green's function for solving the Lippmann-Schwinger equation (2.37) in various situations. The actual numerical evaluation of (2.38) is straightforward but the resonances in the denominator lead to a rather slow convergence behavior for frequencies in the bands.

The Lippmann-Schwinger equation lends itself to various schemes for obtaining approximate solutions. For instance, its behavior for frequencies near a photonic band edge is entirely determined by the DOS [141] so that for increasing distance $\left|\vec{r}-\vec{r}^{\prime}\right|$ it decays with a power law for frequencies in the band and exponentially fast for frequencies in the band gap. As a result, several authors have employed the Green's function technique to derive approximate discrete models for linear and nonlinear photonic crystals with and without waveguiding structures [144-146]. Using the Green's function (2.38) for the strictly periodic linear system, Sakoda et al. [9] have investigated the effects of optical nonlinearities in 2D photonic crystals for $E$-polarization and have suggested enhancements of certain nonlinear properties (see also Section 3). In recent work, the Green's function technique has been applied to waveguiding in 2D photonic-crystal slabs and has provided interesting predictions regarding the effects of fabricational disorder [147] and optical nonlinearities in these systems $[148,149]$.

\subsection{Linear optics—-fabrication and characterization}

\subsubsection{One-dimensional structures}

In the strict sense, a one-dimensional photonic crystal along a certain direction has to be infinitely extended in the two perpendicular directions. Thus, strict one-dimensional photonic crystals are just periodic stacks of quarter-wave layers known as dielectric mirrors for many decades (see Fig. 12(a)). Their generalization, i.e., not quite periodic quarterwave stacks called "chirped mirrors" [150,151], allow for an enlarged freedom in tailoring the group-delay dispersion of the reflected wave. Chirped mirrors are commercially available and are widely used for dispersion compensation 

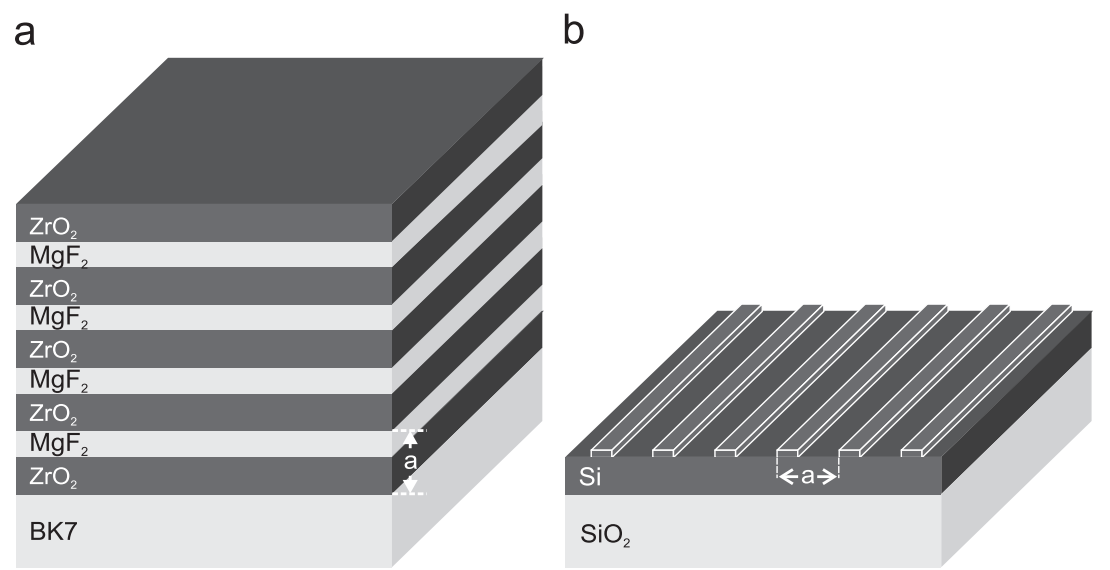

Fig. 12. (a) Scheme of a simple dielectric mirror-a one-dimensional photonic crystal. (b) Scheme of a one-dimensional array of rib waveguides.

of very short laser pulses inside and outside laser cavities. Defect cavities in periodic quarter-wave stacks lead to optical Fabry-Perot filters, which are also readily available commercial products and shall not be discussed here. In the following, we address three more recent examples (i)-(iii) of strictly one-dimensional structures that have been realized experimentally. Thereafter, we briefly discuss one-dimensional structures in a looser sense (see Fig. 12(b)).

(i) Omnidirectional mirrors [152-154]: Consider a periodic quarter-wave stack of alternating low- and high-index materials. For wave propagation normal to the stack, a stop band-a one-dimensional photonic band gap evolves (see Section 2.1.1.1). For propagation of light inside the mirror under an angle with respect to the surface normal, the position of the stop band shifts to the blue. Considering all possible angles and polarizations, no spectral region of overlapping stop bands results, i.e., the structure has no three-dimensional photonic band gap. Clearly, for propagation of light parallel to the dielectric layers, there is not even a stop band. One might be tempted to conclude that this implies that it would be impossible to design a stack of dielectric layers in such a way that it reflects light in a certain frequency interval for all directions of incidence and all polarizations. This conclusion, however, is incorrect as light coming from the outside can only couple to those modes inside the mirror that are above the light line (see Section 2.1.1.1). This is a simple result of the conservation of the wave-vector component parallel to the surface. For the modes below the light line, there may well be a region of overlapping stop bands, provided the index contrast is sufficiently large. In this overlap region, the structure acts as an omnidirectional mirror for an outside observer. This has been demonstrated experimentally [153] for wavelengths between 10 and $15 \mu \mathrm{m}$ using refractive indices of 1.6 and 4.6, respectively. Note, however, that the 3D photon density of states inside the mirror is not zero in the omnidirectional reflectivity band-in contrast to a complete three-dimensional photonic band gap, which may look just the same from the outside in the far field. An emitter with an emission frequency within the high-reflectance band, spatially positioned outside but in the optical near field of the structures interface would sample the inside DOS via the evanescent tails of the modes. Thus, it would behave differently close to an omnidirectional mirror than close to a 3D PBG material.

(ii) Optical analog of electronic Bloch oscillations [155]: Again consider a usual periodic quarter-wave stack of layers with alternating low and high refractive index. In addition, a constant gradient of the refractive index shall be superimposed. This situation is analogous to an electron in the periodic potential of nuclei in a crystal, subject to a homogeneous electric field. In the time domain, a wave packet gets accelerated due to the gradient, is Bragg reflected, e.g., from the right-hand side (RHS) of the BZ to the left-hand side (LHS), which changes its propagation direction, it is again accelerated, Bragg reflected, etc. The corresponding real-space oscillation of the wave packet is known as Bloch oscillation. It has been observed experimentally by time resolving a femtosecond pulse transmitted by such a structure. The frequency-domain counterpart is the Wannier-Stark ladder, i.e., the photonic bands break up into a series of equidistant discrete levels, corresponding to peaks of high transmittance.

(iii) Bragg and anti-Bragg structures [156]: Consider a periodic arrangement with lattice constant $a$ of thin layers of a material exhibiting a Lorentz-oscillator resonance [142] in its refractive index. If the Lorentz-oscillator resonance wavelength $\lambda$ coincides with the Bragg wavelength, i.e., if $a=\lambda / 2$ ("Bragg structure"), the Lorentz oscillators can 
efficiently couple by the radiation field, leading to a broadening of the Lorentz resonance, e.g., seen in the transmittance. For $a=\lambda / 4$ ("anti-Bragg structure"), an avoided crossing of the Bragg and Lorentz resonance results. Both aspects have been observed on multiple quantum well structures in the GaAs/AlGaAs material system, in which the GaAs 1s excitonic resonance at low temperatures plays the role of the Lorentz oscillator and the AlGaAs barrier acts as a dielectric spacer [156].

The surprising physics of a strictly one-dimensional photonic crystal comprising magneto-dielectric layers will be discussed in Example 7 of Section 5.4.1.

In a looser sense, one-dimensional photonic crystals can also be composed of three-dimensional building blocks, periodically arranged along one dimension with lattice constant $a$. Examples are fiber Bragg gratings [157], linear arrays of coupled micro-cavities [158], coupled resonator optical waveguides [159] (CROW), or one-dimensional lattices of rib waveguides. Historically, the concept of one-dimensional waveguide lattices was introduced theoretically in 1965 [160] (actually for optical fibers), first experiments were reported some years later [161]. Recent reviews are given in Refs. [162,163], even more recent results can be found in a special issue [164]. Such structures can easily be fabricated by standard microfabrication approaches, e.g., in the AlGaAs material system or in polymers. Note that the propagation direction of light and the reciprocal lattice vectors are orthogonal to each other here (see Fig. 12(b)), whereas they are parallel in the above strictly one-dimensional systems (i)-(iii). Clearly, the rib waveguides can couple via the evanescent tails of their confined optical fields, forming a model system for a tight-binding band structure [8]. The coupling strength can conveniently be controlled by the spatial separation between adjacent rib waveguides. Such arrangements are often referred to as discrete systems because normally one has a continuous coordinate perpendicular to the propagation direction of light, which is now replaced by a discrete waveguide number. Thus, many effects known from "normal" linear and nonlinear optics can nicely be revisited facing their discrete counterpart. For the example of linear optical diffraction, discrete systems have even more to offer because diffraction can be engineered [165,166], whereas it is fixed in continuous systems. There, it is just proportional to the square of the transverse wave number, leading to normal diffraction. In the discrete counterpart, the cosine-shaped tight-binding energy dispersion translates into normal diffraction for "small" transverse wave numbers, i.e., $k_{x}$ given by $0<\left|k_{x} a\right|<\pi / 2$, no diffraction for $\left|k_{x} a\right|=\pi / 2$, and negative diffraction or anomalous diffraction for $\pi / 2<\left|k_{x} a\right|<\pi$. Experimentally, $k_{x}$ can be varied by changing the angle of incidence $[165,166]$. Imposing an additional linear refractive-index gradient perpendicular to the waveguides (e.g., induced by a variable temperature gradient) again creates a situation similar to the Bloch oscillations of electrons (see discussion above). For the waveguide lattices [167,168], the beauty is that the spatial field distribution can be mapped out experimentally, which is not easily possible for the above strictly one-dimensional situation. Recently, also Zener tunnelling [169] into higher photonic bands and the discrete analog of the Talbot effect revivals [170] have been observed along these lines. Much of the interest in this field, however, is directed towards nonlinear optics, especially to solitary waves $[162,163]$ (also see Section 3).

\subsubsection{Two-dimensional structures}

In the strict sense, a two-dimensional photonic crystal in the $x y$-plane has to be infinitely extended along the $z$-direction. In this geometry, light is obviously not guided at all along $z$. In other words, a block of material with an ordered array of pores much longer than the wavelength of light and all oriented along $z$ could do the job. Indeed, electrochemically grown nanoscale pores (i.e., air cylinders) in semiconductors and metals are known for more than half a century [171,172]. In 1994, self-ordered pores with domains in the micron range were observed in porous alumina [173]. The domain sizes can dramatically be increased to several square inches (!) by pre-patterning with either electron-beam lithography or nano-indentation. Regarding photonic-band-gap materials, crystalline silicon is of particular interest. Corresponding pioneering work on the anodization of n-type silicon in hydrofluoric acid has been published in Refs. [174,175], recent reviews are given in Refs. [176-179]. An example is depicted in Fig. 13. Waveguide structures are, e.g., discussed in Ref. [179].

Most "two-dimensional photonic crystals", however, are slab waveguide structures in which the light is guided along the $z$-direction by total internal reflection (see Section 2.1.1.1). A slab waveguide can either be achieved by a free-standing thin membrane (symmetric) or by a high-index film on a low-index substrate in air (asymmetric). Such slab-waveguide structures appear more suitable for applications in telecommunications as many other telecommunication devices, such as, e.g., edge emitting semiconductor lasers, are slab-waveguide structures already. They can be fabricated by standard electron-beam lithography. Typically, high-index semiconductors like $\mathrm{Si}, \mathrm{GaAs}$, InP or other III-V compounds are employed. In fewer cases, also low-index polymeric structures and glasses (e.g., $\mathrm{Nb}_{2} \mathrm{O}_{5}$ ) have 

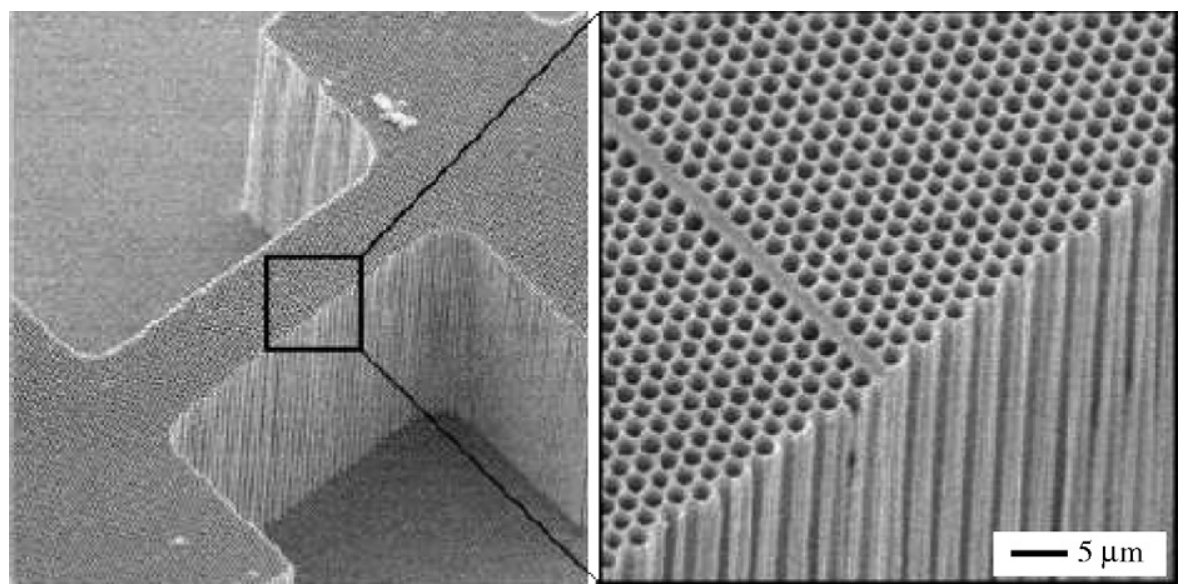

Fig. 13. Electron micrograph of a two-dimensional hexagonal porous silicon structure with a lattice constant of $a=1.5 \mu \mathrm{m}$ and a pore radius of $r=0.68 \mu \mathrm{m}$, fabricated by anodization of p-type silicon after pre-patterning. The close-up shows a waveguide fabricated in this structure. Reprinted with permission from [180]. Copyright (2001) by Wiley VCH.

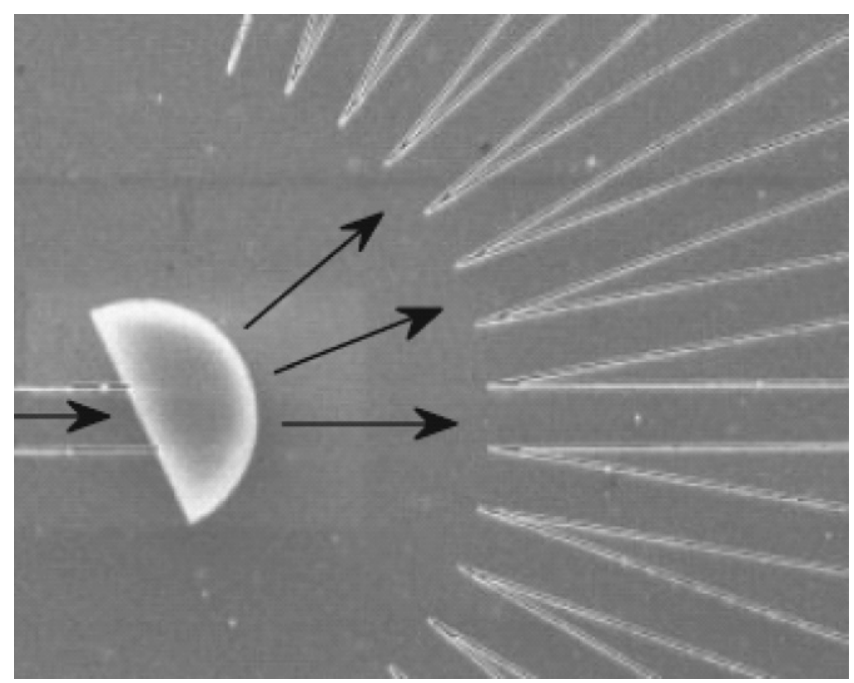

Fig. 14. Electron micrograph of a planar micro-spectrometer based on the superprism effect of a two-dimensional photonic-crystal slab waveguide structure. Reprinted with permission from [184]. Copyright (2003) by Wiley VCH.

been investigated (see, e.g., the review [181]). Here, structures with complete two-dimensional photonic band gaps (i.e., for both $E$-like and $H$-like polarization) are neither known nor likely to occur. Regarding high-index two-dimensional slab waveguide photonic crystals, such complete band gaps have only been realized experimentally rather recently [182]. The current focus is on functional defect and/or waveguide structures based on this platform. Let us consider a few selected examples.

The superprism effect addressed above (see Section 2.1.2.1) is a unique feature of photonic crystals, arising from the special shape of the iso-frequency contours in reciprocal space. Based on the corresponding frequency dependence, a wavelength multiplexer was designed and implemented in a planar geometry [183] (see Fig. 14), operating with a wavelength swing of $10^{\circ}$ per $20 \mathrm{~nm}$ for wavelengths around $1300 \mathrm{~nm}$. Compared to a conventional one-dimensional grating, the angular resolution is five-fold improved [184].

Small, high-finesse cavities are of interest for ultra-compact filters [185-187], low-threshold lasers [188-191], nonlinear optics [192], and quantum optics [193-195] (“cavity quantum electrodynamics"). They are sometimes referred 
to as "photonic atoms" [158]. Two parameters of a cavity are of crucial importance, namely its quality factor and its mode volume. Recall that the quality factor $Q$ of any damped resonance is given by the ratio of the resonance frequency to its spectral width. Equivalently, in the time domain, one has $Q$ "round-trips" in the cavity within the lifetime of the photon. For reference, quality factors as large as $Q=10^{10}\left(Q=10^{7}\right)$ have been obtained from whispering gallery modes in fused-silica (polystyrene) microspheres with diameters on the order of $1 \mathrm{~mm}(30 \mu \mathrm{m})$ around $0.6 \mu \mathrm{m}$ vacuum wavelength [196,197]. The second important quantity of a cavity is its mode volume $V$. It can be defined [188] via $\int \vec{D}(\vec{r}) \cdot \vec{E}(\vec{r}) \mathrm{d} V=\max (\vec{D}(\vec{r}) \cdot \vec{E}(\vec{r})) V$. For comparison, the fundamental mode in a dielectric cube of side length $l$, coated with a thin film of ideal metal (see Section 5.1) has a mode volume of $V=(1 / 2)^{3} l^{3}=(\sqrt{3} / 4)^{3} \lambda^{3} \approx 0.08 \lambda^{3}$, with the material wavelength $\lambda$. The ratio $Q / V$ determines the strength of various cavity interactions [198]. For a given geometry and for fixed wavelength, reduction of the mode volume typically tends to increase radiation losses, hence deteriorating the quality factor. Thus, obtaining large $Q / V$-ratios is not a trivial enterprise. What design criterion can be used to obtain large $Q / V$-ratios for cavities in two-dimensional photonic-crystal slab waveguide structures, provided that absorption losses are negligible and fabrication tolerances are absent? Ref. [187] offered an intuitive and very successful strategy: We have seen in Section 2.1.1.1 that in-plane wave-vector components outside the vacuum light cone cannot couple to the modes outside the slab. Those inside the light cone can couple. Thus, the magnitude of the latter determines the losses. For a perfectly periodic photonic crystal, a snapshot of the electric field could be cosine-shaped $\propto \cos \left(\vec{k}_{0} \cdot \vec{r}\right)$ in real space, corresponding to sharp maxima in reciprocal space $\propto \delta\left(\vec{k} \pm \vec{k}_{0}\right)$. We assume that $\vec{k}_{0}$ is outside the light cone. Suppose, for example, that we just eliminate a row of $N$ adjacent air holes from the periodic structure (see Fig. 15). In Fourier space, this abrupt spatial change (from the two-dimensional photonic crystal to bulk material) introduces a broad distribution of in-plane wave-vector components with maxima centered

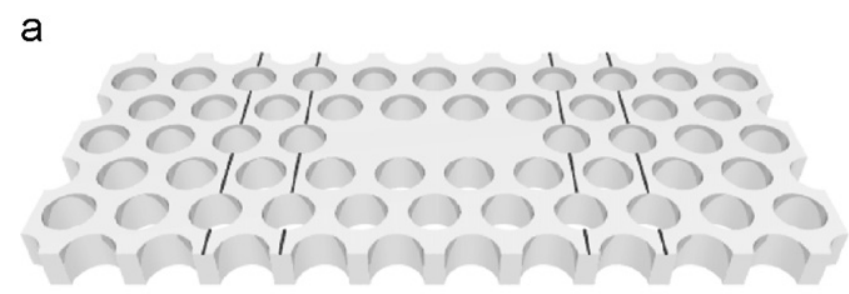

b
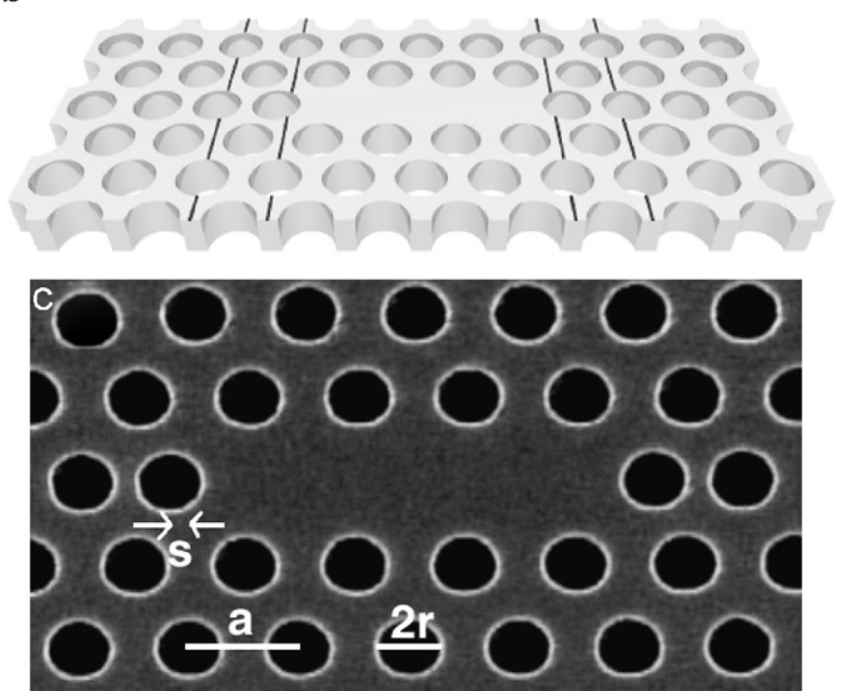

Fig. 15. Illustration of the optimization guideline "... the light should be confined gently in order to be confined strongly" [187] that has led to cavities in two-dimensional photonic-crystal slab waveguides with large $Q$-values and very large $Q / V$-ratios. (a) Undisturbed photonic crystal with cavity, (b) holes in the nearest and next-nearest neighborhood are slightly shifted (solid lines are guides to the eyes). (c) Electron micrograph of a GaAs cavity structure fabricated by electron-beam lithography along the lines of (b). Part (c): Reprinted figure with permission from [195]. Copyright (2004) by Nature Publishing Group. 
around $\vec{k}= \pm \vec{k}_{0}$. In particular, large components inside the light cone are generated, which can couple to the outside world, thus limiting the $Q$-factor. In contrast, if the spatial modulation is smooth ("adiabatic"), e.g., Gaussian in shape, the maxima in the Fourier spectrum become narrow Gaussians, largely reducing the wave-vector components inside the light cone. In other words "the light should be confined gently in order to be confined strongly" [187]. Following this design rule, which is illustrated in Fig. 15, measured quality factors as large as $Q=65,000$ have been reported in silicon structures with mode volumes of $0.07 \mu \mathrm{m}$ at $1.6 \mu \mathrm{m}$ vacuum wavelength. More recently, these values have been further improved to an impressive measured quality factor of $Q=600,000[199,200]$ and even $Q \approx 1,000,000$ [201]. The corresponding calculated value is yet larger with $Q=20,000,000$, indicating that imperfections do play a role in the experiments [201].

Such cavities with high $Q$-values and small mode volume allow for studying the strong coupling of a single mode of the electromagnetic field to a single two-level system emitter [195]. Apart from being a nice quantum optical paradigm, this system is of potential interest for quantum information processing: A single absorbed pump photon would completely change its optical properties. The two-level emitter can be realized by self-organized 25-nm diameter InAs quantum dots embedded in a free-standing GaAs photonic-crystal plane. These quantum dots can have very large electric-dipole moments $d_{2 \mathrm{LS}}$, enabling strong coupling of light and matter. Even at quantum dot densities around $300-400 \mu \mathrm{m}^{-2}$, the broad inhomogeneous distribution of excitonic transition energies from these quantum dots and the small homogeneous linewidth at low temperatures make it extremely unlikely to find within one cavity two quantum dots with nearly similar frequencies, both resonant with the cavity mode. In fact, it is even very unlikely to have just one quantum dot resonant with the cavity mode (measured $Q \approx 20,000$ at about $1.2 \mu \mathrm{m}$ vacuum wavelength, and calculated $V=0.04 \mu \mathrm{m}^{3}$ ). Thus, a very large number of cavities had to be investigated [195]. The experimental signature of strong coupling is a double peak structure in the photoluminescence spectrum, resulting from the avoided crossing of the quantum dot exciton and the cavity resonance (the vacuum Rabi splitting [198]). By changing the sample temperature from 13 to $29 \mathrm{~K}$, the spectral position of the exciton resonance can be shifted across the more or less fixed cavity mode, revealing (see Fig. 16) the avoided crossing with an estimated Rabi splitting energy of $170 \mu \mathrm{eV}(0.192 \mathrm{~nm}$ in wavelength). This value, which scales $\propto d_{2 \mathrm{LS}} / \sqrt{V}$, is indeed larger than the mean of the cavity linewidth $=\hbar \omega / Q \approx 78 \mu \mathrm{eV}$ and the spontaneous emission linewidth of the quantum dot-a necessary condition for strong coupling. If the cavity resonance was spectrally much narrower than the quantum dot, a Fano-lineshape would result in the optical spectrum (see Section 5.3).

A functional element in which some degree of losses from a cavity is even desired is shown in Fig. 17. Here, light propagating along a straight waveguide can couple to the nearby cavity via the evanescent tails of the modes, provided its wavelength is resonant with the cavity. The abrupt spatial change of just a single enlarged air hole leads to considerable coupling to modes outside the slab. In wavelength-division multiplexing in telecommunications, such a device can act as an add/drop filter where certain wavelength channels from the waveguide are emitted from the cavity into the direction normal to the photonic-crystal plane (drop filter) or the other way around (add filter) —as experimentally demonstrated in Ref. [185].

a

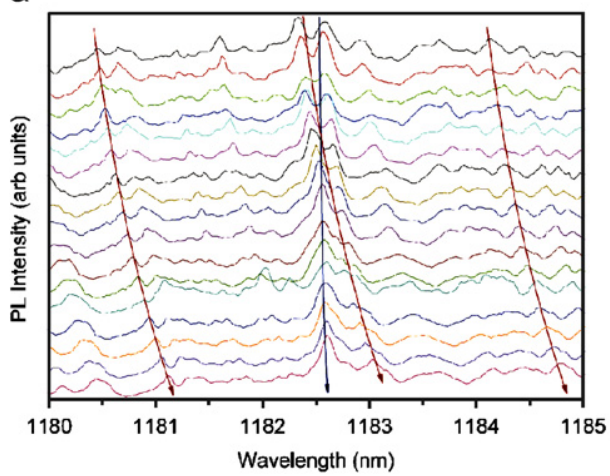

b

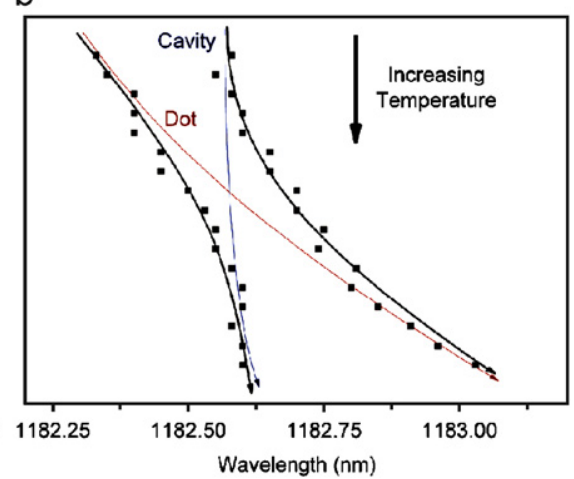

Fig. 16. (a) Measured photoluminescence spectra (low excitation) for sample temperatures from $13 \mathrm{~K}$ (top) to $29 \mathrm{~K}$ (bottom) in $1 \mathrm{~K}$ steps, and (b) analysis of the peak positions deduced from (a). Reprinted figure with permission from [195]. Copyright (2004) by Nature Publishing Group. 


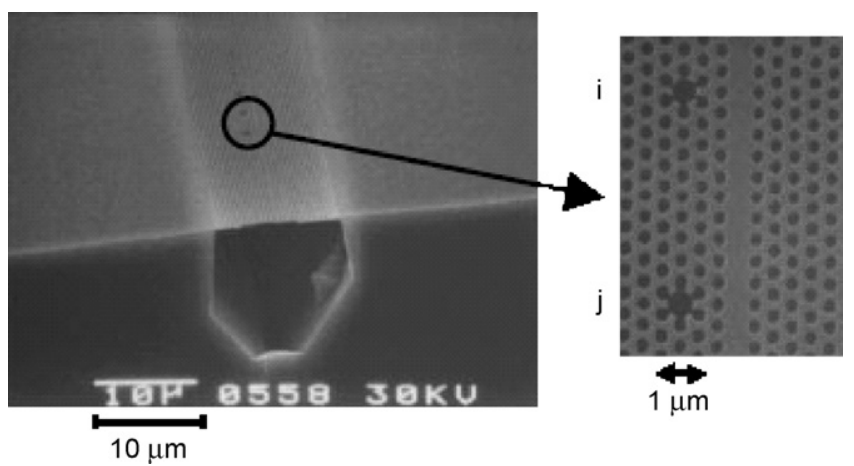

Fig. 17. Electron micrograph of a InGaAsP-based add/drop filter, based on a cavity coupled to a waveguide in a two-dimensional photonic-crystal slab waveguide, fabricated by electron-beam lithography. Reprinted figure with permission from [185]. Copyright (2000) by Nature Publishing Group.

Considerable research effort has been devoted to designing and fabricating waveguides, waveguide bends, splitters, crossings, etc. in small spaces (see Section 2.1.3). Indeed, the vision of compact photonic circuitry has been one of the motivations of the field of photonic crystals from the start. However, to date the bottom line is that, regarding losses, two-dimensional photonic-crystal slab waveguide architectures are presently only as good as usual high-index rib waveguides-despite the fact that the latter are conceptually inferior: Latest results reported losses of $3 \mathrm{~dB} / \mathrm{cm}$ for a W1 waveguide in a photonic crystal [202], while conventional high-index rib waveguides perform as good as $3-4 \mathrm{~dB} / \mathrm{cm}$ [203].

This might further improve in the near future as new design optimization algorithms find their way into the photoniccrystal community. These algorithms are based on the idea of inverse design and are powerful tools for the optimization of specific tasks, e.g., transmission enhancement of waveguide bends. Mainly two methods are used so far: the level-set approach [205] and topology optimization [206]. The latter technique has already left the conceptual stage and resulted in greatly enhanced performance of $90^{\circ}$ waveguide bends in a triangular lattice [204]. This is especially interesting as a proof of concept as $90^{\circ}$ bends are not commensurate with the triangular lattice and conventional design based on intuition leads to strong losses per bend. Furthermore, the optimized structures look somewhat disordered and could possibly never have been guessed by intuition (see Fig. 18).

It is clear from the complicated structure in Fig. 18 that further improvement of the optical properties goes hand in hand with advances in fabrication technology. At the present stage, concentrating on aspects that are unique for photonic crystals seems indicated. One such unique aspect of photonic-crystal architectures is the ability to tailor the dispersion relation of light. In particular, light with small group velocities can be realized. The most direct way to investigate this is to launch a pulse into a waveguide and study the resulting temporal evolution of the local field. This has been accomplished [207] by positioning the tip of a near-field optical microscope into the evanescent tail of the electromagnetic mode of the slab waveguide structure. The locally collected light can be superimposed onto a frequency-shifted reference field (heterodyning). The resulting interference gives access to both local amplitudes and phases. In this fashion, group velocities smaller than $c_{0} / 1000$ have been reported [207] (Fig. 19).

\subsubsection{Three-dimensional structures}

To date, the "holy grail" of flexible and inexpensive fabrication of large-scale, high-quality, three-dimensional photonic-band-gap materials, and complex photonic architectures based on this platform, remains elusive. Thus, the field of three-dimensional photonic crystals is dominated by fabrication and characterization issues rather than physics or device applications. We start our discussion with direct fabrication techniques and continue with a variety of promising alternative approaches, aiming at describing the present state-of-the-art.

2.2.3.1. Directfabrication methods. Nanofabrication of two-dimensional structures using electron-beam or UV lithography is a standard procedure known from microelectronics. Conceptually, the "straightforward" way to extend this to three dimensions is to use a layer-by-layer approach: In a first step, the surface of a wafer is processed by the 

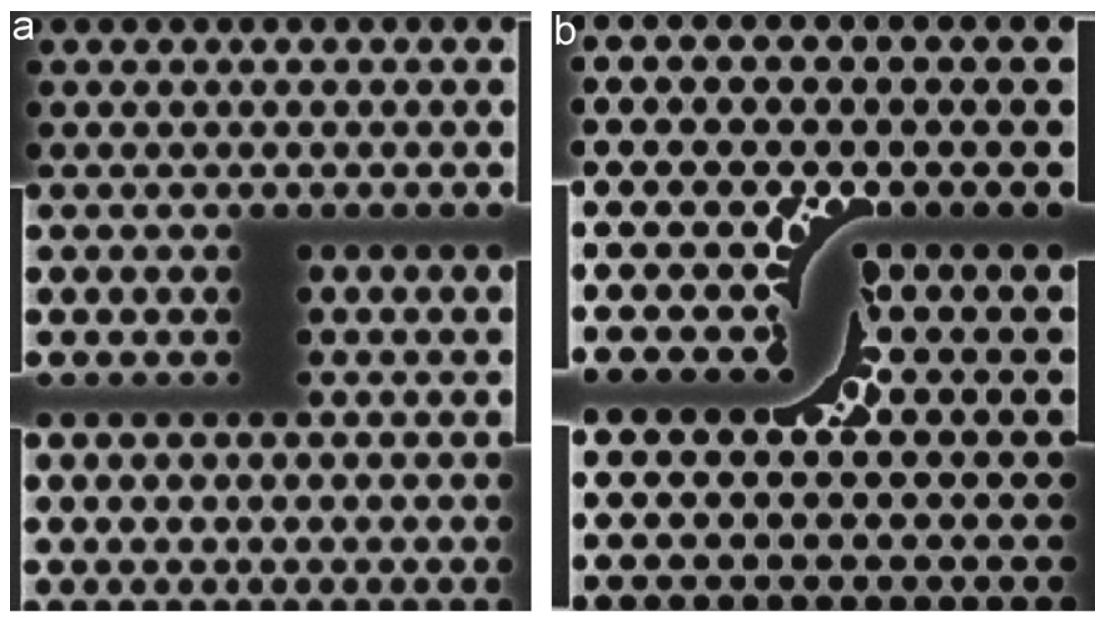

C

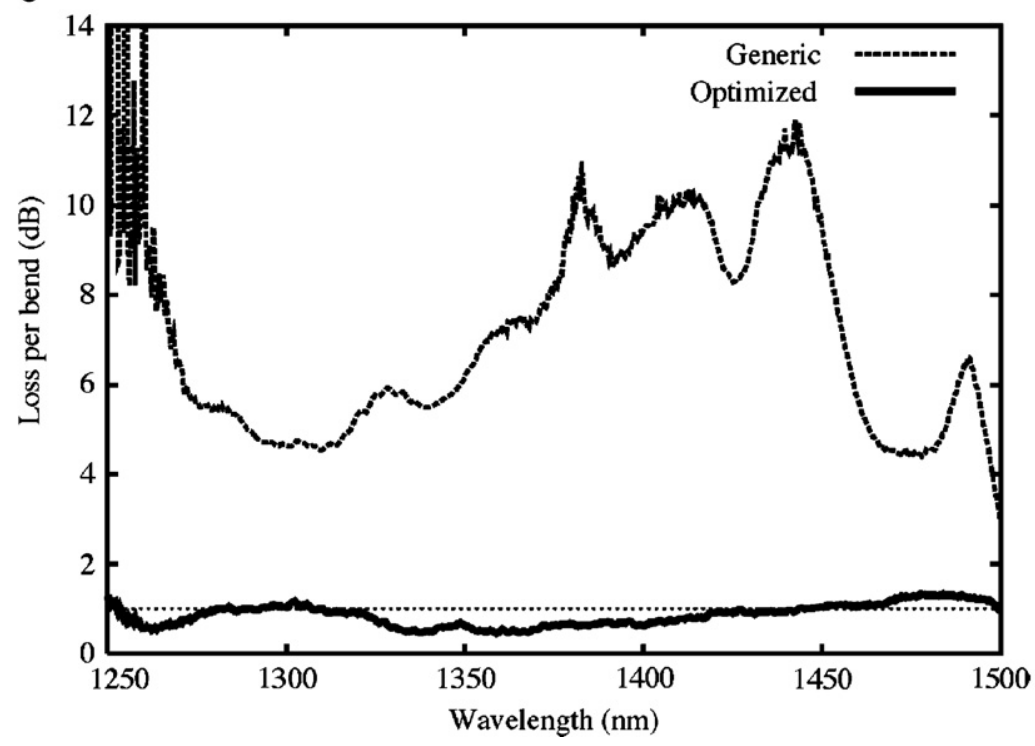

Fig. 18. Electron micrographs of a double $90^{\circ}$ bend in a two-dimensional triangular SOI photonic crystal fabricated by electron-beam lithography. (a) Conventional design, (b) topology optimized design. (c) Comparison of the measured bend loss for the fabricated generic and the optimized waveguide structure. Taken from [204].

above-mentioned techniques. The next layer is then added either by deposition and subsequent patterning of a dielectric film or by bonding two pieces of the wafer at temperatures of several hundreds degrees Celsius ("wafer fusion"), followed by removal of one of the substrates. This procedure is repeated several times until the desired number of layers is reached.

The woodpile (or logpile) structure [209] is ideally suited for such a sequential approach. Here, layers of straight parallel rods with a center-to-center distance $a$ are stacked to a three-dimensional lattice. Adjacent layers have an orientation of the rods rotated by $90^{\circ}$ and second-nearest neighboring layers are shifted by a distance of $a / 2$ perpendicular to the rod axes. This stacking sequence repeats itself after every four layers with a lattice constant $c$. For $c / a=\sqrt{2}$, this lattice exhibits an face centered cubic (fcc) unit cell with a two-rod basis and can be derived from a diamond lattice by replacing the $\left(\begin{array}{lll}1 & 1 & 0\end{array}\right)$ chains of lattice points with rods. Woodpiles can exhibit a complete three-dimensional photonic band gap between the second and the third band. For 30\% silicon volume filling fraction, a gap/midgap ratio of $18 \%$ results. The inverse structure has a gap/midgap ratio of $25 \%$ at $82 \%$ silicon volume filling fraction. This excellent gap/midgap ratio can further be increased up to $28 \%$ by using slightly elliptical rods with an aspect ratio of 5/4 [209]. 
a

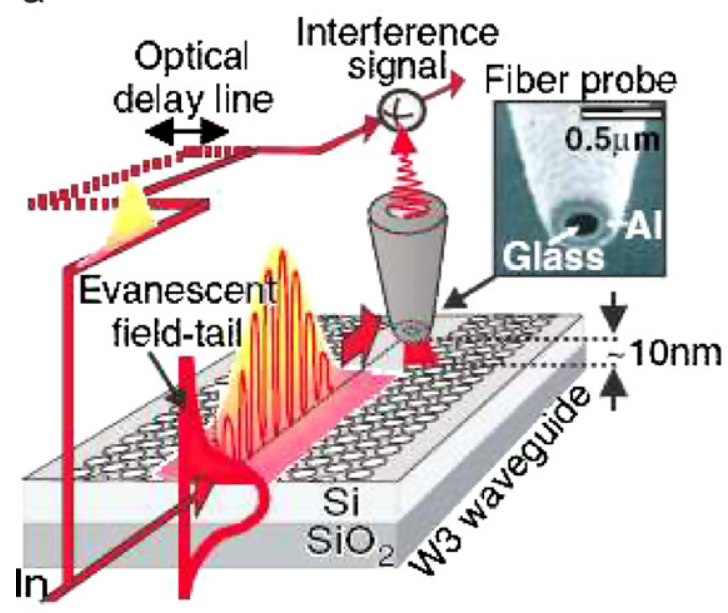

b

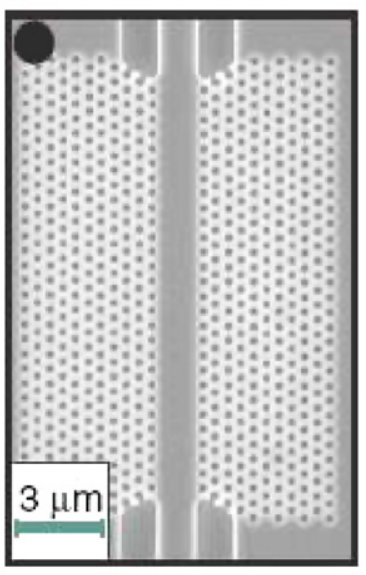

Fig. 19. (a) Scheme of the near-field optical experiment. (b) Top-view electron micrograph of one of the W3 silicon-on-insulator waveguide structures investigated (a short one), fabricated by electron-beam lithography. Reprinted figure with permission from [207]. Copyright (2005) by the American Physical Society.

In practice, three-dimensional photonic crystals fabricated by this approach [208,210-216] are limited by the complexity of the fabrication process to only a few lattice constants in the vertical direction, e.g., 2 or 3 lattice constants (i.e., 8 or 12 layers) of the woodpile structure. Additionally, a high degree of mechanical precision is required to avoid offsets between the different layers. This can be achieved by laser-beam-assisted alignment. It is immediately apparent that such a sequential procedure is quite time consuming, expensive, and technically very demanding. Nevertheless, beautiful and "heroic" experiments have been performed along these lines.

Ref. [208] achieved photonic band gaps at telecommunication wavelengths using the III-V direct-gap semiconductors GaAs or InP, more recently even in combination with an optically active defect [214]. Corresponding electron micrographs are shown in Fig. 20, measured optical spectra in Fig. 21. The measured transmittance reveals $40 \mathrm{~dB}$ suppression in the photonic band gap for just two lattice constants. To allow for a direct comparison with the theoretical expectation for the finite structure shown in Fig. 21, we depict corresponding scattering-matrix calculations (see Section 2.1.4.2) in Fig. 22. The structural parameters and the thickness of the structure are taken from the experiment. Note, that there are still differences between experiment and theory, especially in the expected spectral position. Ref. [215] presented corresponding silicon-based woodpile structures at telecommunication wavelengths with up to 5 layers $(\approx 1$ lattice constant) with a rod alignment precision of $10 \%$ of the lattice constant. Metallic woodpile photonic crystals made along these lines will be discussed in Section 5.1.

Another structure [217] that is very well suited for the layer-by-layer approach has successfully been realized at telecommunications wavelengths in Ref. [216]. This layered structure is essentially a stack of two different types of two-dimensional photonic crystals: Air holes in a dielectric plate and its complement, i.e., dielectric rods in air. The structure on a fcc lattice exhibits a complete three-dimensional photonic band gap between the second and the third bands. For $21 \%$ volume filling fraction silicon in air, a gap/midgap ratio of $21 \%$ results. Importantly, the structure allows for arbitrarily designed defects to be introduced in any layer.

Let us briefly mention two further and completely different direct fabrication approaches for three-dimensional structures: (i) The first one is based on the modulation of the electrochemical current during growth of pores in porous silicon [179] (see Section 2.2.2). In this fashion, simple-cubic structures with a complete three-dimensional photonic band gap with $4 \%$ gap/midgap ratio around $3.2 \mu \mathrm{m}$ wavelength have been demonstrated [218]. Notably, 10 lattice constants are possible in the $z$-direction. However, the extension of these results to shorter wavelengths appears to be difficult. Furthermore, the incorporation of cavities and waveguides in three dimensions seems challenging. Alternatively, electrochemically grown two-dimensional structures can be post-processed by focused-ion-beam milling [219]. (ii) The second one is based on glancing-angle deposition and allows for large-scale square-spiral photonic crystals, proposed in Ref. [220]. In this approach, complex three-dimensional structures can be fabricated by combining 
A
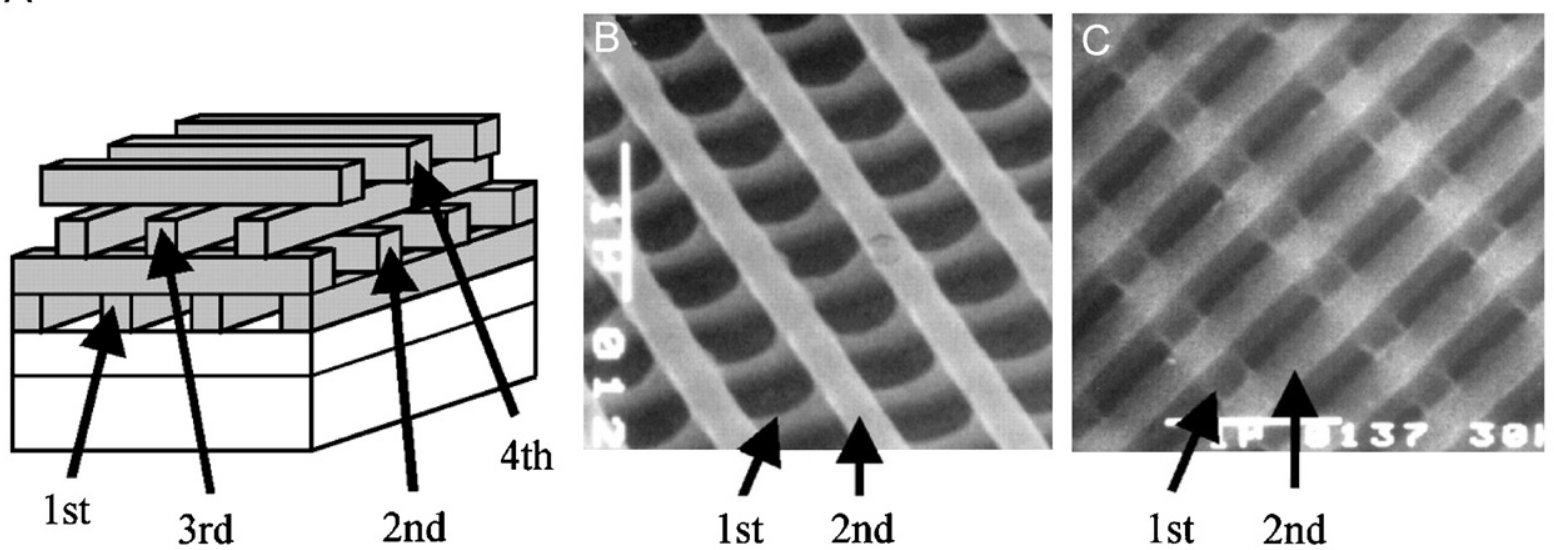

D

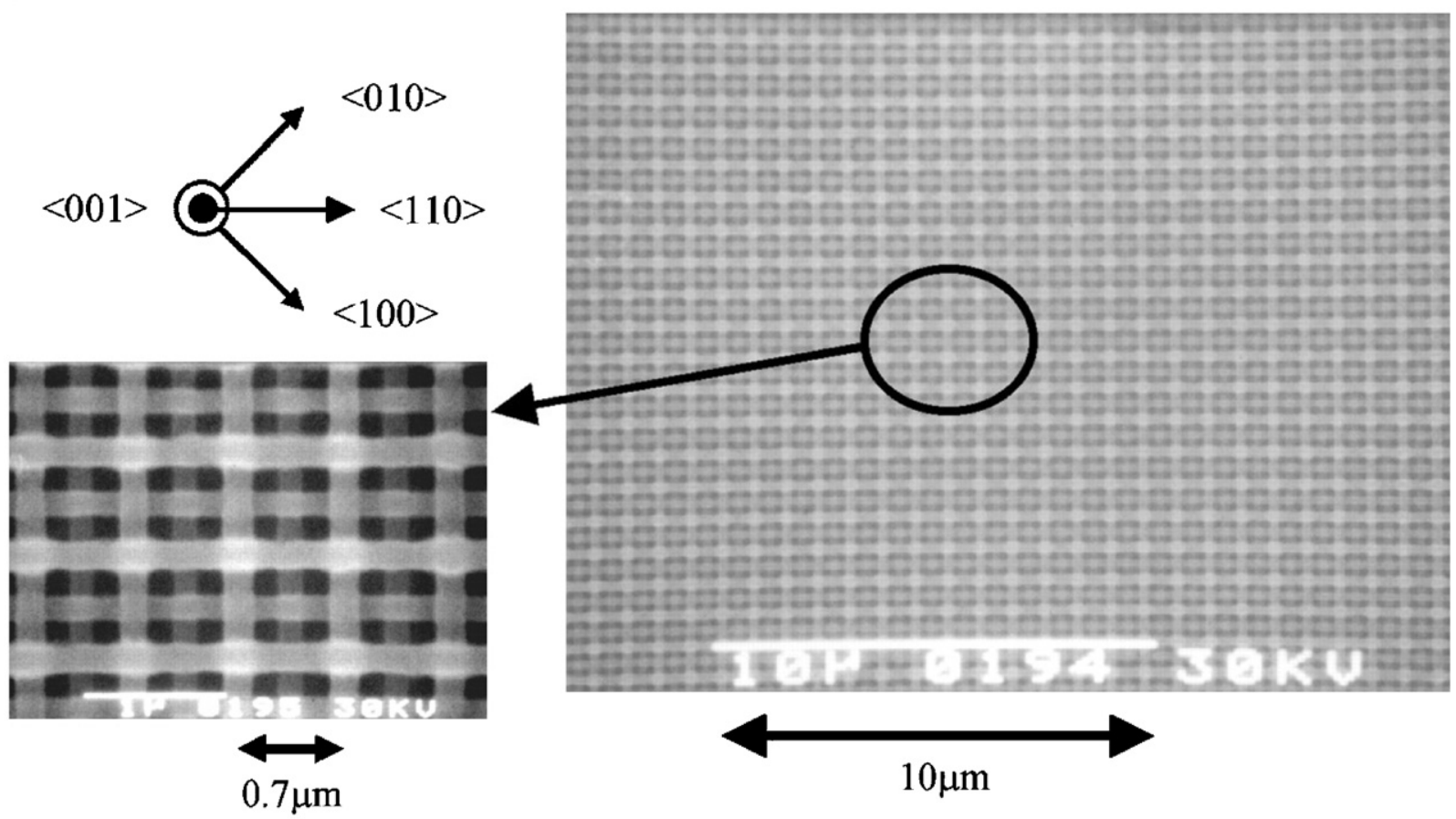

Fig. 20. Scheme and electron micrographs of woodpile photonic crystals fabricated by the layer-by-layer approach. Reprinted figure with permission from [208]. Copyright (2003) by AAAS.

oblique vapor deposition and precisely controlled motion of a two-dimensionally pre-patterned substrate [221,222]. The resulting square spiral posts form a tetragonal lattice. The positive (inverse) structure has a maximum band gap between the fourth and the fifth band with 15\% (24\%) gap/midgap ratio for 30\% (21\%) silicon volume filling fraction. First experiments have been reported on silica [223], silicon [224], and $\mathrm{Alq}_{3}$ structures [225]. Again, the incorporation of waveguide and/or defect architectures seems difficult. However, the structural chirality of three-dimensional spiral structures can lead to pronounced circular dichroism for propagation of light along the spiral axis [225,226]. The square spiral structure as well as other spiral structures are also amenable to direct laser writing (see Section 2.2.3.4).

2.2.3.2. Inverse opals. Opals, a dense packing of sub-micron diameter silica spheres, are naturally occurring threedimensional photonic crystals known as semiprecious jewellery stones [227]. One knows from solid-state physics, that 
a

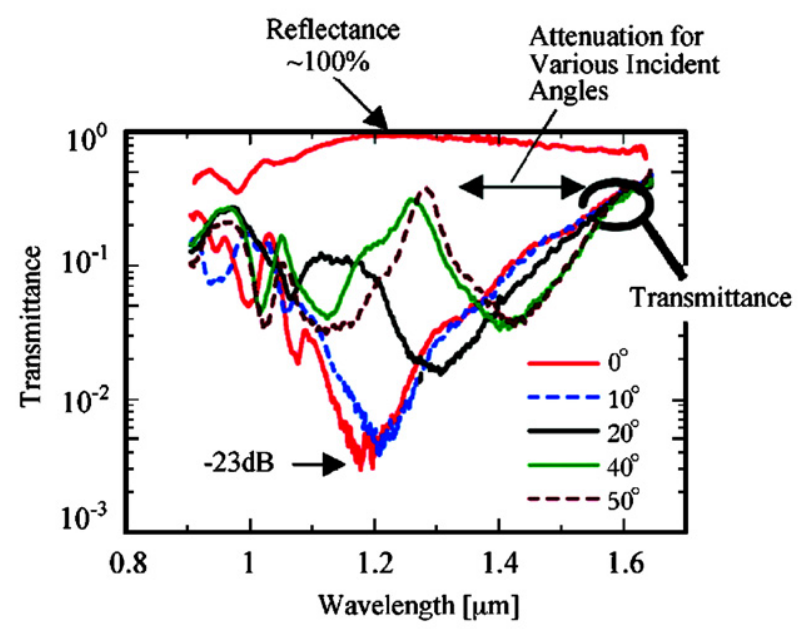

b

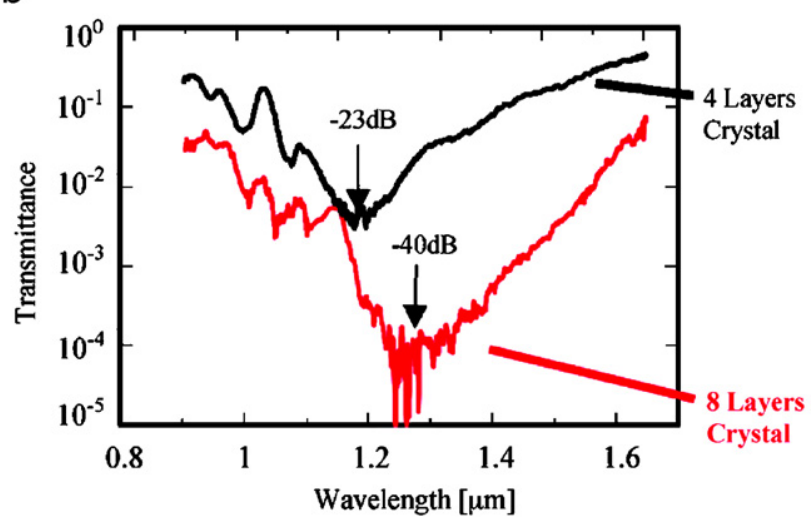

Fig. 21. Transmittance spectra of the structure shown in Fig. 20. Reprinted figure with permission from [208]. Copyright (2000) by AAAS.

two inequivalent dense packings of spheres exist, the fcc and the hexagonal-closed-packing (hcp) arrangement. Artificial opals can for example be fabricated via colloidal self-assembly of silica or polystyrene spheres using gravity sedimentation techniques [228,229], convective self-assembly [230,231] or evaporation-induced self-assembly [232-234]. In principle, these techniques allow for facile and inexpensive large-area template fabrication. For both fcc and hcp opals, neither the refractive index of silica $(n \approx 1.5)$ nor the limit $n \rightarrow \infty$ leads to a complete three-dimensional photonic band gap. Inverse opals result from infiltration of the air voids in an opal with a material of index $n$ and subsequent removal of the original spheres, e.g., by wet chemical etching with HF or plasma etching for silica and polystyrene, respectively. It turns out that inverse fcc opals exhibit a complete photonic band gap between the 8th and the 9th band for $n \geqslant 2.8$ [235,236,39], inverse hcp opals between the 16th and the 17th band for $n \geqslant 3.1$ [39,237]. With the refractive index of silicon, fcc opals exhibit a gap/midgap ratio of $4.5 \%$ for hypothetical full infiltration and can posses a gap/midgap ratio of up to $8.6 \%$ for partial infiltration [39]. At first sight, it does not seem possible to incorporate intentional cavities and/or defects into opaline structures. Nevertheless, several different approaches have been demonstrated [238-240], mostly, however, leading to rather large dimensions and moderate final quality.

In more detail, high-quality opal templates are the crucial starting point. Commercially available off-the-shelf spheres have standard deviations in their diameter on the order of $5 \%$ or more. This introduces sufficient disorder to close the anticipated complete photonic band gap $[39,237]$. The dedicated synthesis of silica spheres can reduce the standard deviation to below $2 \%$ [234]. Unfortunately, the fcc close packing of spheres, which corresponds to an ABC ABC ... sequence, and the hcp dense packing, corresponding to an $\mathrm{AB} A \mathrm{AB}$... sequence, are energetically very nearly similar. To make the two inequivalent for typical sphere diameters suitable for photonics, the long-range interaction between 


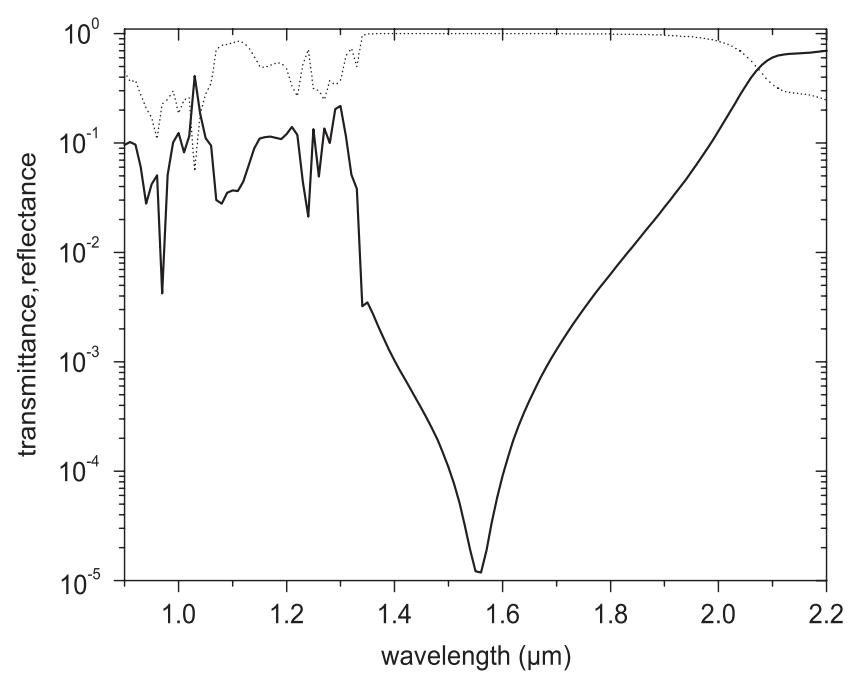

Fig. 22. Normal-incidence transmittance (solid) and reflectance (dotted) spectrum for parameters corresponding to the finite structure shown in Fig. 21 , calculated via the scattering-matrix approach. Slight deviations from the measured spectra suggest a still significant influence of imperfections in the actual structure.

spheres in plane "A" and in plane " $\mathrm{C}$ " would have to span about $1 \mu \mathrm{m}$ distance or more. As a result, stacking faults start to develop from the third layer on, i.e., already within the first lattice constant of the fcc structure. Stacking faults and hcp dense packing can in principle be avoided by growing opals not perpendicular to the $\left\{\begin{array}{lll}1 & 1 & 1\end{array}\right\}$ plane-a plane common to both, fcc and hcp-but starting with the $\left\{\begin{array}{lll}10 & 0\end{array}\right\}$ plane. This can be achieved via microstructured substrates, but requires commensurate sizes of the microstructure and the spheres [241].

To mechanically stabilize the opal template prior to the high-index material infiltration, a thin layer of silica is deposited via chemical vapor deposition (CVD) or atomic-layer deposition (ALD) [242]. This introduces necks at the former singular contact points of adjacent spheres. Accidentally, these necks tend to increase the size of the photonic band gap [39]. Broadly speaking, CVD and ALD are distinct from evaporation of materials because CVD and ALD specifically lead to deposition of material at the surfaces, hence avoiding clogging of channels. For the deposition of silica $\left(\mathrm{SiO}_{2}\right)$, the sample surfaces are first coated with a thin layer of water $\left(\mathrm{H}_{2} \mathrm{O}\right)$, e.g., by letting nitrogen gas that has bubbled through a water bottle, flow through the sample. Next, using silicon tetrachloride $\left(\mathrm{SiCl}_{4}\right)$ as the precursor, the chemical reaction $\mathrm{SiCl}_{4}+2 \mathrm{H}_{2} \mathrm{O} \rightarrow \mathrm{SiO}_{2}+4 \mathrm{HCl}$ only takes place at the surfaces and is automatically terminated when the water is consumed [242]. Depending on the process parameters, atomic layers can be deposited in this fashion. Next, silicon is deposited via CVD. Typically, low-pressure flow reactors are advantageous compared to static CVD, as growth parameters can be controlled during deposition and deposition can be stopped after the desired film thickness is reached. Disilane $\left(\mathrm{Si}_{2} \mathrm{H}_{6}\right)$ is most commonly used as a precursor for photonic-crystal fabrication, as decomposition temperatures are lower than for silane and the excess $\mathrm{H}_{2}$ passivates the dangling bonds. At typical temperatures around $450{ }^{\circ} \mathrm{C}$, the effective reaction at the sample surfaces is $\mathrm{Si}_{2} \mathrm{H}_{6} \rightarrow 2 \mathrm{Si}+3 \mathrm{H}_{2}$. Deposition is stopped when the opal pores are completely infiltrated. Finally etching with aqueous $\mathrm{HF}$ removes the $\mathrm{SiO}_{2}$. Examples of resulting structures are shown in Figs. 23 [243] and 24 [244,245]. Inverse opals have been fabricated with numerous materials, examples can be found in recent reviews [246-248]. Metallic (tungsten) inverse opals will be discussed in Section 5.1.

Let us finally mention that, in microelectronics, CVD and ALD are well-established processes for the threedimensional deposition of many different dielectrics as well as metals, see, e.g., Refs. [249-252]. CVD and ALD can also be used for the inversion and/or double inversion of photoresist photonic-crystal templates to be discussed next.

2.2.3.3. Holographic lithography. The main idea of holographic lithography [253-255] is to expose a thick-film photoresist to one or several standing wave patterns originating from the interference of a number of laser beams. There are two distinct strategies to realize this. In the first approach, a single three-dimensional standing wave pattern 

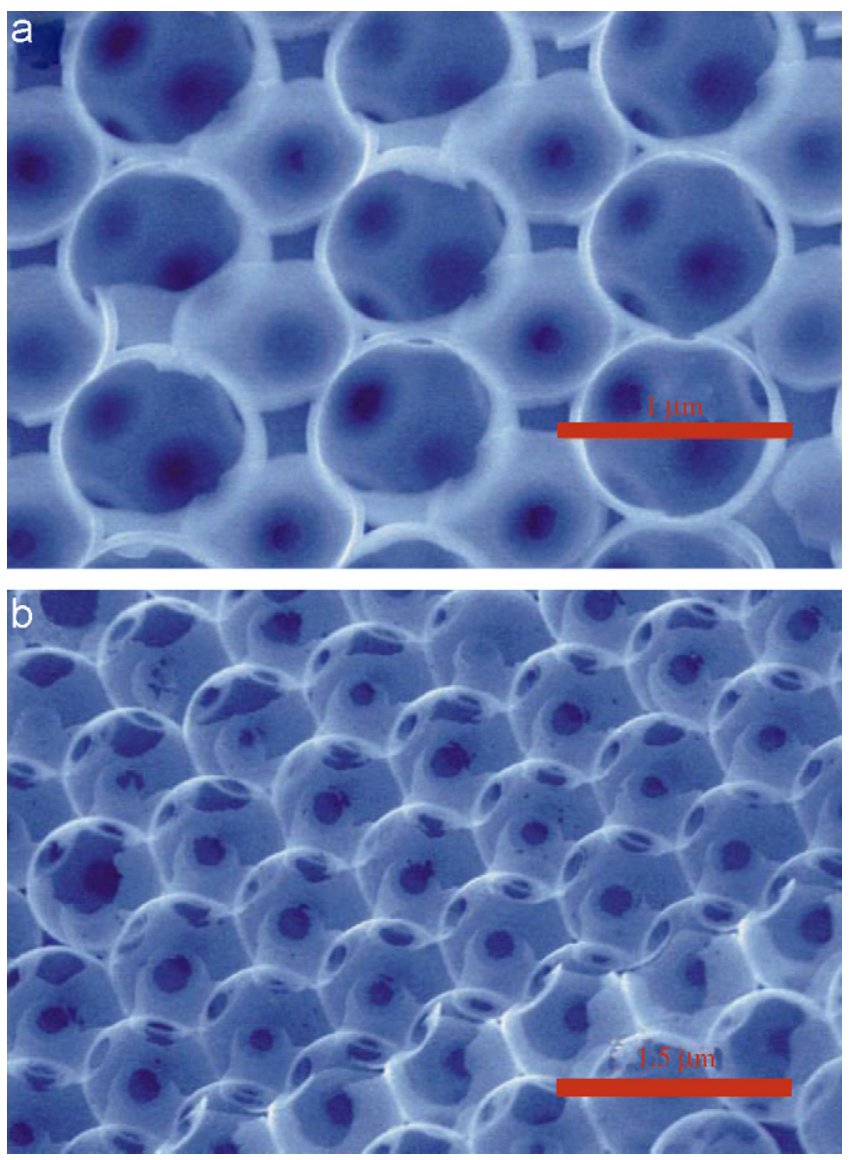

Fig. 23. Electron micrograph of a silicon inverse opal exhibiting a complete photonic band gap around $1.5 \mu \mathrm{m}$ wavelength. Reprinted figure with permission from [243]. Copyright (2000) by Nature Publishing Group.

is realized from the simultaneous interference of at least four laser beams. The physics of such "light lattices" has first been discussed in the context of atomic physics years ago [256]. In the second approach, the photoresist is sequentially exposed to different standing wave patterns, e.g., by three two-beam interferences.

For both cases as well as for direct laser writing (see the next section), the commercially available photoresist SU-8 from MicroChem is suitable and quite common. It consists of the epoxy EPON SU-8 and a photoinitiator both dissolved in gamma-butyrolactone (GBL) and can be spun onto a glass substrate. Upon one-photon absorption by near-UV light (350-400 nm wavelength), or, alternatively, upon two-photon absorption (700-800 nm wavelength), the photoinitiator generates an acid. The spatial acid concentration is an image of the irradiation dose. In a post-exposure bake the latent picture is converted into a cross-linking density by cationic polymerization during this thermal treatment. ${ }^{5}$ The crosslinking degree determines the solubility in the "developing" solvent. GBL or another appropriate solvent is used in this step. Thus, sufficiently illuminated resin remains ("negative" photoresist) whereas under-exposed resin is washed away. The remaining material, therefore, has a shape that follows the surface for the threshold irradiation which is the boundary between over- and under-exposed regions. Effectively, the interference pattern is stored in a digitized form: It is a porous air-polymer-structure showing the iso-dose surface for the threshold dose value. In reality, however, shrinkage of the SU-8 during development can distort the structures. An isotropic shrinkage just reduces the lattice constant, an asymmetric shrinkage would distort the crystal symmetry. Thus, measures against the deteriorating effects of shrinkage have to be taken (see the discussion below).

\footnotetext{
${ }^{5}$ The post-exposure bake can, however, also be accomplished via the laser itself taking advantage of thermal effects during exposure, with beneficial effects for the resolution [257].
} 

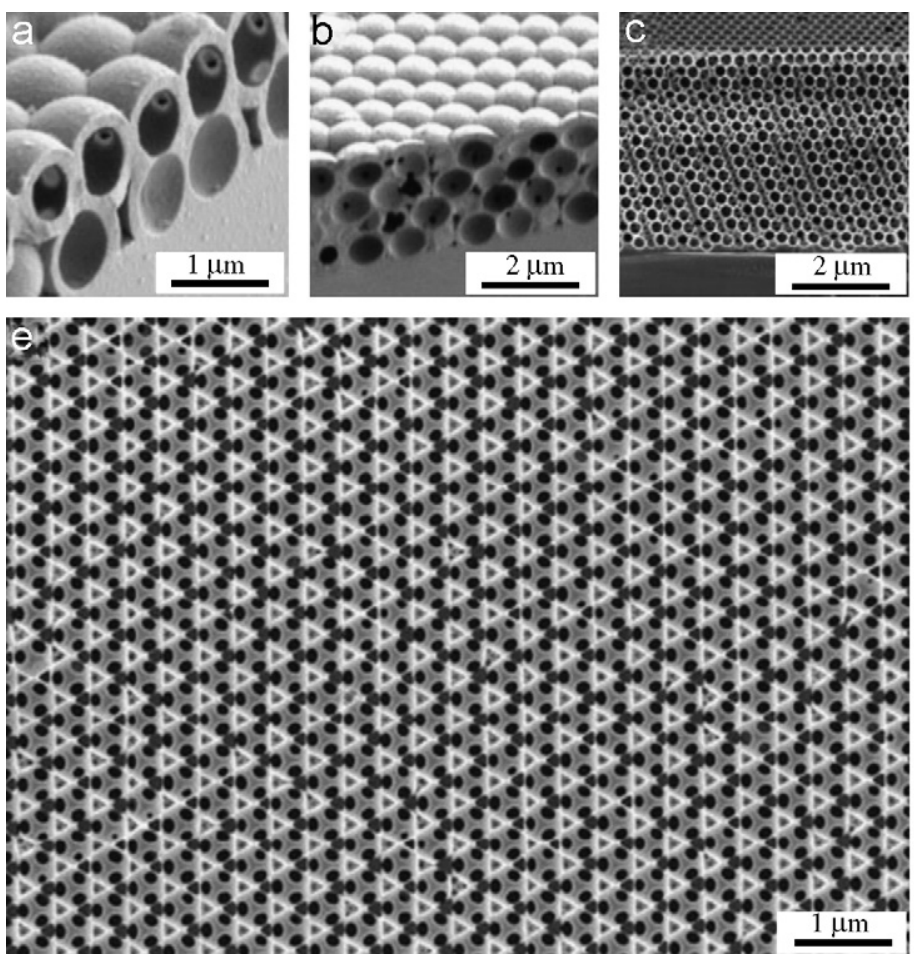

Fig. 24. Electron micrograph of a silicon inverse opal. The original opal has been fabricated on a pre-patterned silicon substrate. Reprinted figure with permission from [244]. Copyright (2001) by Nature Publishing Group.

The local exposure dose is proportional to the local light intensity $I(\vec{r})$ and proportional to the exposure time. Let us start our discussion with the case of a single four-beam exposure. Here, $I(\vec{r})$ results from the superposition of four light beams (plane waves) and can be expressed as

$$
I(\vec{r}) \propto\left|\sum_{n=1}^{4} \vec{E}_{n} \mathrm{e}^{\mathrm{i}\left(\vec{k}_{n} \vec{r}-\omega t\right)}\right|^{2}=\sum_{n=1}^{4} \sum_{m=1}^{4} a_{n m} \mathrm{e}^{\mathrm{i} \vec{G}_{n m} \vec{r}}
$$

with the reciprocal lattice vectors

$$
\vec{G}_{n m}=\vec{k}_{n}-\vec{k}_{m}
$$

and the form factors

$$
a_{n m}=\vec{E}_{n} \cdot \vec{E}_{m}^{*} \text {. }
$$

The latter result from the relative amplitudes (or intensities) and polarizations of the four beams. It is clear that the reciprocal lattice vectors determine the translational symmetry of the crystal. Four wave vectors of light allow for three linearly independent reciprocal lattice vectors, hence allowing for three-dimensional crystals. The shape of the motif (see Section 2.1.1.1) results from the form factors. In general, lattice and motif have different point symmetries, resulting in some overall crystal symmetry common to both. At present, there are mainly two four-beam configurations under discussion: The "two-planes" geometry has two pairs of beams travelling in planes perpendicular to each other (Fig. 25(b)). In this geometry, the substrate has to be passed by two beams. Moreover, rather small lattice constants result since beams are counter-propagating. The "umbrella-like" configuration, in which three beams (\#2, \#3, \#4) are equally distributed on a cone with a certain apex angle $\gamma$ and the fourth beam (\#1) is directed along the axis of the cone (Fig. 25(a)), is more convenient experimentally.

While the above equations suggest that any of the 14 Bravais lattices in three dimensions should easily be accessible via holographic lithography, refraction of the incident laser beams at the air/photoresist interface inhibits that: If the angles 


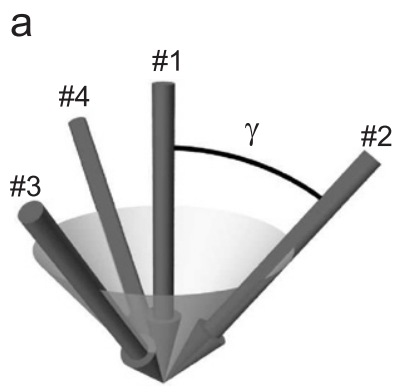

b

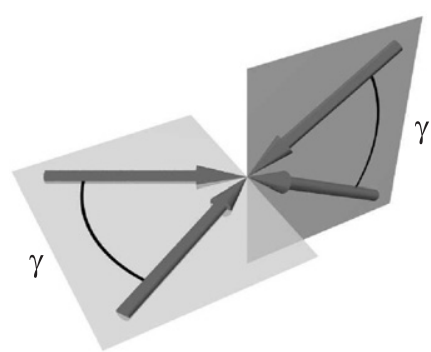

Fig. 25. (a) The umbrella-like configuration: The central beam (\#1) is along the axis of a cone with apex angle $\gamma$; three side beams (\#2, \#3, \#4) are equally distributed on the cone. (b) The two-planes geometry: Two pairs of counter-propagating beams in two planes perpendicular to each other. Taken from Ref. [258].

have, for example, been properly adjusted to give fcc translational symmetry in air, an undesired trigonal translational symmetry results within the photoresist. Even for grazing incidence, desired symmetries such as for example fcc cannot be achieved [255]. However, by adding an appropriately shaped dielectric object on the air/photoresist interface, refraction at this interface can be reduced and, e.g., fcc translational symmetry becomes possible [255]. The measured transmittance spectra reveal a pronounced stop band around $700 \mathrm{~nm}$ wavelength, which agrees well with band structure calculations [255] (see Section 2.1.4.1).

The total number of free parameters in the holographic lithography of three-dimensional photonic crystals with four beams is 20: For each of the four beams one has 2 parameters for the orientation of the beam for fixed exposure wavelength, 1 parameter for the beam amplitude, and 2 additional parameters for the generally elliptical polarization. Even if the translational symmetry is already fixed by the choice of reciprocal lattice vectors, 12 free parameters remain. On the one hand, this offers an enormous degree of flexibility for designing the motif. On the other hand, this high-dimensional parameter space makes it truly difficult to actually identify parameter sets that eventually lead to complete three-dimensional band gaps and, at the same time, correspond to a sufficiently large contrast of the corresponding interference pattern. As already mentioned above, the umbrella geometry is to be preferred from an experimental viewpoint. For the two-planes geometry structures have been proposed with gap/midgap ratios as large $23 \%$ if made of silicon $[259,260]$. To date, none of these proposals has been realized experimentally. For the umbrella geometry, parameters were independently suggested in Refs. [258,261]. The approach is to take advantage of the fact that functions (essentially sums consisting of trigonometric functions) of known crystal symmetry-which is generally different from the translational symmetry discussed above-are available from crystallography for all of the 230 space groups in three dimensions $[258,262]$. These functions are given in the crystal coordinate system (generally not a Cartesian system), whereas the above interference pattern (2.39) is quoted in the Cartesian laboratory frame. Thus, in a first step, the interference pattern has to be represented in the corresponding crystal system. In the second step, a comparison of the coefficients delivers the form factors $a_{n m}$ (if possible), required for a certain space group. Band structure calculations along the lines described in Section 2.1.4.1 with variable filling fractions follow. It turns out that an infinite number of combinations of parameters deliver exactly the same form factors. This freedom can be used to independently optimize another very important aspect for the practical realization, namely the interference contrast. The resulting algebraic equation can be solved analytically [258]. One of the solutions is a crystal with fcc translational symmetry and rhombohedral crystal symmetry, which delivers a moderate gap/midgap ratio of $5.7 \%$ for infiltration with silicon at a volume filling fraction of $37 \%$ (see Fig. 26). This complete gap between the second and the third band (see Fig. 27) is expected to be quite robust against fabricational tolerances. Interestingly, this structure-which roughly resembles the famous "Yablonovite"-is balanced. For example, for 50\% filling fraction, this means that the structure is simply identical to its inverse (apart from a shift in space). Thus, complete band gaps can be achieved along these lines for inversion of the templates as well as for double inversion-leaving both options available [258]. This also remains valid for filling fractions other than $50 \%$, in which case, however, the exposure dose has to be compensated accordingly.

In addition, another attractive solution with simple cubic translational and crystal symmetry has been found as well $[258,261]$. It has a complete gap between the fifth and the sixth bands with a gap/midgap ratio of $11 \%$ for infiltration 

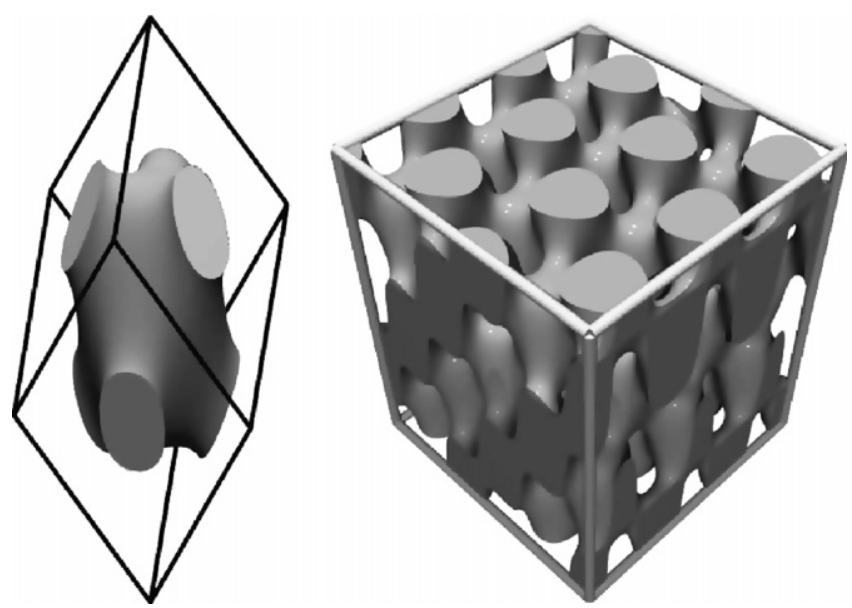

Fig. 26. LHS: Motif of the rhombohedral structure within the rhombohedral unit cell for a silicon volume filling factor of $37 \%$. RHS: Fragment of the corresponding crystal structure. Taken from Ref. [258].

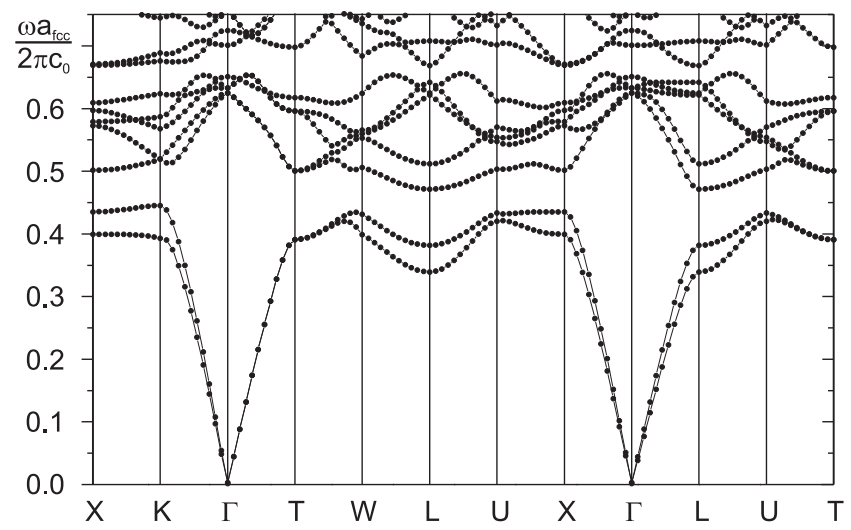

Fig. 27. Band structure of the photonic crystal shown in Fig. 26. Note that there is a complete photonic band gap between the 2nd and the 3rd bands as well as another complete gap between the 7th and the 8th band. Taken from Ref. [258].

with silicon at a volume filling fraction of $24 \%$. For an interference contrast optimized for linearly polarized beams, the corresponding interference pattern is given by [258]

$$
I(\vec{r}) \propto 3.67+\cos (2 \pi x / a)+\cos (2 \pi y / a)+\cos (2 \pi z / a)
$$

with interference contrast $I_{\max } / I_{\min }=(3.67+3) /(3.67-3) \approx 10$ and lattice constant

$$
a=\frac{\lambda \sqrt{3}}{2} .
$$

$\lambda$ is the exposure wavelength inside the photoresist.

Eq. (2.42) for the simple cubic case is just the sum of three cosines, each corresponding to a single two-beam interference. Thus, a pattern identical in shape to (2.42) can be achieved not only by a four-beam interference using the umbrella configuration but alternatively by three sequential two-beam exposures from three different directions [90] (see Fig. 28). For maximized interference contrast, each beam of such a pair needs to be $s$-polarized, leading to

$$
I(\vec{r}) \propto 3+\cos (2 \pi x / a)+\cos (2 \pi y / a)+\cos (2 \pi z / a)
$$


a
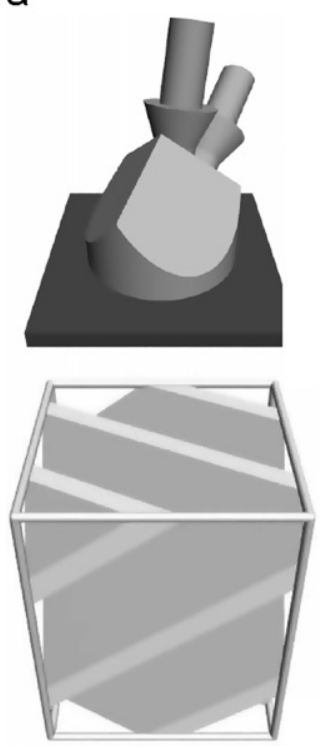

b
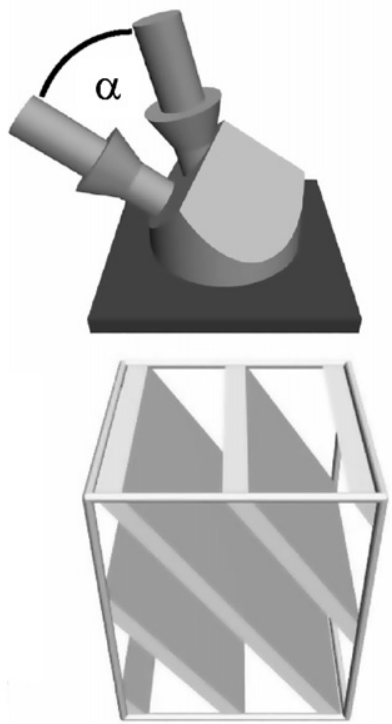

C
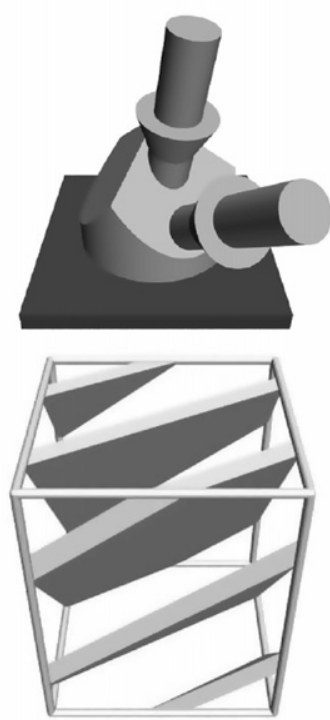

d

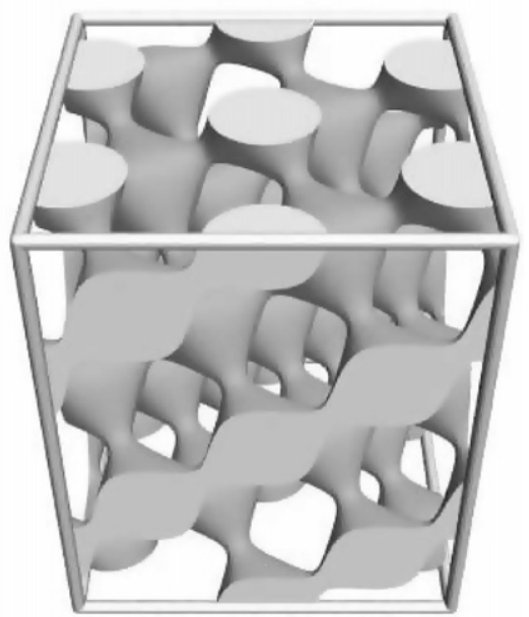

Fig. 28. Illustration of the multiple-exposure scheme with the beams coupled into the resist through a corner-cube prism [255]. (a)-(c) The resist is sequentially exposed to three two-beam interference patterns, only differing in a rotation about a three-fold axis. Each individual exposure would result in a lamellar structure as shown below. By varying the angle $\alpha$ between the two beams in each pair while maintaining the bisector, the lattice constant $a$ can be varied conveniently. (d): Resulting three-dimensional simple cubic structure after accumulating the three exposures (a)-(c). Taken from Ref. [90].

with interference contrast $I_{\max } / I_{\min }=(3+3) /(3-3)=\infty$ and lattice constant

$$
a=\frac{\lambda}{2 \sin (\alpha / 2)} \text {. }
$$

For the special case of $\alpha=\gamma_{\mathrm{sc}}=\arccos (1 / 3)$ from Ref. [258], (2.45) reduces to (2.43). The advantage of the three sequential two-beam exposures is that the photonic-crystal lattice constant $a$ cannot only be scaled via the exposure wavelength $\lambda$ (which is inconvenient experimentally as the laser and the photoresist have to be changed) but also via the angle $\alpha$ between the beams of a pair-largely improving the flexibility of the approach [263,261]. The multiple exposure scheme is illustrated in Fig. 28 with the threefold axis along the $z$-axis, whereas it is along the [111]-direction in Eq. (2.44) for convenience. Similarly, the rhombohedral (Yablonovite-like) structure addressed above [258] can also be fabricated using three sequential two-beam exposures [90].

One variation of the overall scheme of holographic lithography is to generate the different beams by a single beam impinging onto a periodic phase mask on top of the photoresist film to be exposed [264,265] via diffraction from the mask. Parameters eventually leading to a complete 3D photonic band gap after silicon backfilling have been presented theoretically in Ref. [266]. Here, the phase mask creates five (rather than four) interfering beams, leading to the desired diamond crystal symmetry-without employing the two-planes geometry. A second variation employs a corner cube with a chopped-off tip [267] (compare corner cube in Fig. 28). Here, the different partial waves are generated by refraction at the four surfaces. This variation can obviously also be extended to more than four interfering beams by using more than four surfaces of a suitably shaped dielectric object (e.g., five surfaces from a four-sided pyramid with a chopped-off tip). For both of these variations, phase masks and dielectric objects, interferometric stability can be guaranteed for much longer times than in arrangements with freely propagating beams. This aspect is an important advantage for (long) continuous-wave exposures but is hardly relevant for exposures with a single short pulse. A drawback of both variations is that they are less flexible than free-space setups-an aspect that is relevant for research but irrelevant for mass fabrication.

2.2.3.4. Direct laser writing. Holographic lithography is capable of producing samples with defect-free areas of several square millimeters (with the potential for much more) and offers a high flexibility in tailoring the interior of the unit cell. 
However, due to the fabrication principle, these structures are strictly periodic, i.e., cavity and waveguide structures cannot easily be included in a controlled fashion. Therefore, a second complementary technique is required to inscribe functional elements into photonic crystals provided by holographic lithography. An excellent candidate for this task is direct laser writing (DLW) through multi-photon polymerization [268-270]. Alternatively, DLW can also be used to write entire photonic crystals.

In more detail, in multi-photon DLW, a photoresist exhibiting an intensity threshold for exposure is illuminated by laser light whose photon energy is insufficient to expose the photoresist by a one-photon absorption process. If this laser light is tightly focused into the resist, however, the light intensity inside a small volume element ("voxel") inclosing the focus may become sufficiently high to exceed the exposure threshold by multi-photon processes. By scanning the focus relative to the photoresist, in principle, any three-dimensional connected structure consisting of these voxels may be written directly into the photoresist. In analogy to holographic lithography, only doubly connected structures can actually be fabricated. The size and shape of the exposed voxels depend on the isophotes of the microscope lens and the multi-photon exposure threshold of the photosensitive medium. The isophotes in the vicinity of the geometrical focus typically exhibit a near-ellipsoidal shape and voxels with a lateral diameter as small as $100 \mathrm{~nm}$ and an aspect ratio of about 2.7 can be realized [87,271]. Generally, liquid as well as solid photoresists can be employed in DLW. Solid resists, such as SU-8 (see above), do not only make the sample handling much more comfortable, but also offer a higher degree of freedom in the scanning pattern, since successively written structures do not have to be interconnected immediately.

A large variety of different structures has been fabricated along the lines of DLW. Here, we focus on structures for photonics, i.e., structures with sufficiently small lattice constants for gaps at telecommunication wavelengths [87,271]. Owing to its simple geometrical structure, the layer-by-layer or woodpile photonic-crystal structure (also see Section 2.2.3.1) is ideal for DLW. Here, the woodpile rods are built up of joining individual voxels. Fig. 29 shows electron micrographs of corresponding structures. It should be noted that these samples are sufficiently large in area for optical measurements as well as for potential future devices. With the optimized writing procedure used in Ref. [87], the exposure time for the photonic crystal shown in Fig. 29(a) is only about $25 \mathrm{~min}$.

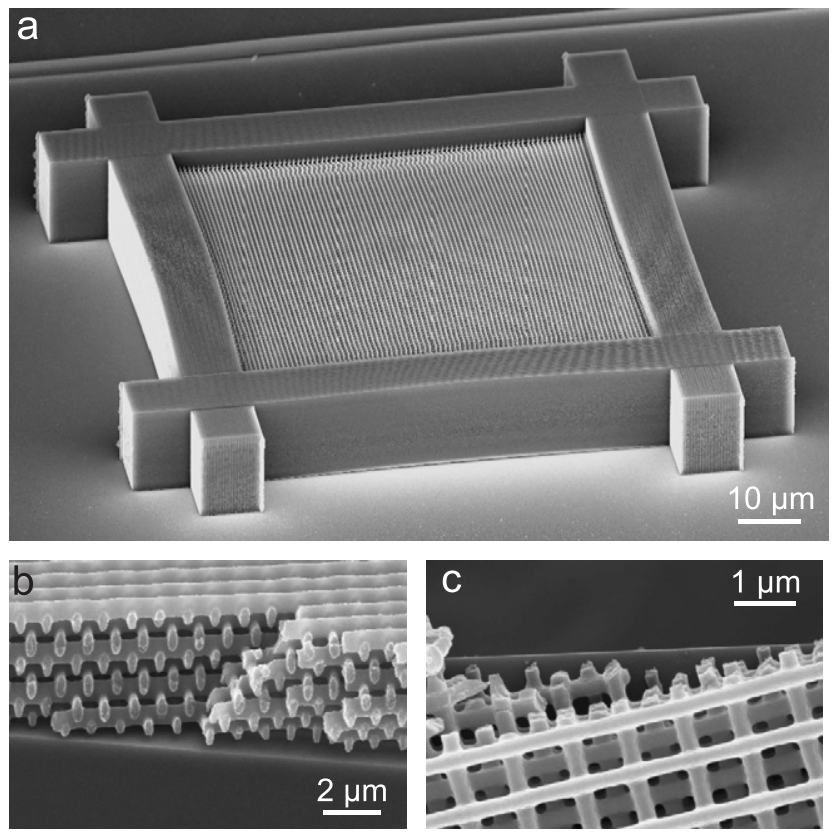

Fig. 29. Three-dimensional photonic crystals fabricated by direct laser writing (DLW). (a) Layer-by-layer structure with 40 layers and a massive wall that prevents bending and reduces distortions due to polymer shrinkage during polymerization, completely fabricated by DLW. (b) Side and (c) top view of a different broken sample with 12 layers, illustrating the sample quality obtained with the DLW process. Taken from [87]. 
a

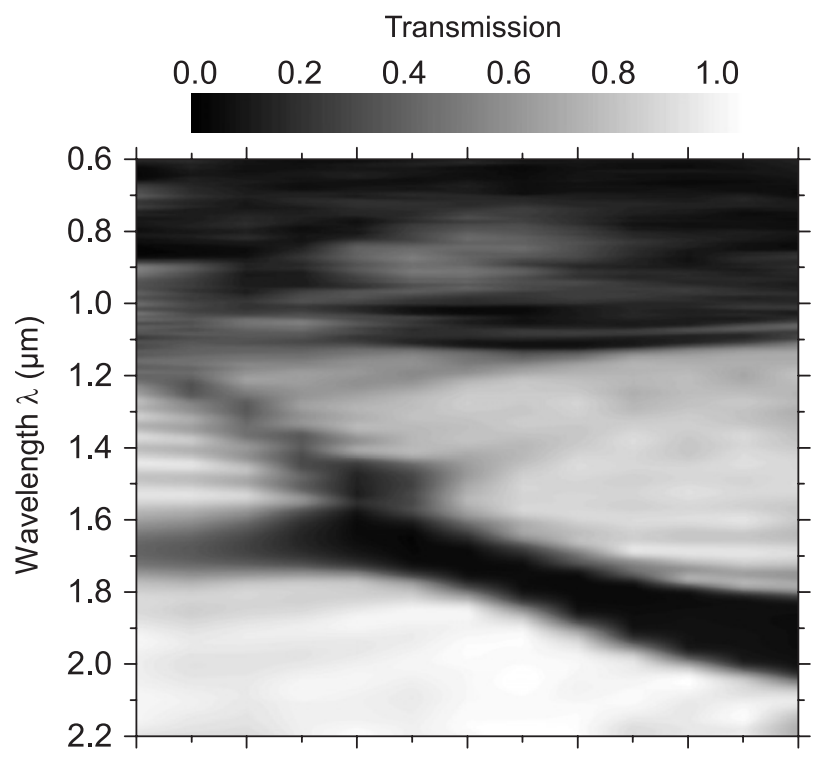

b

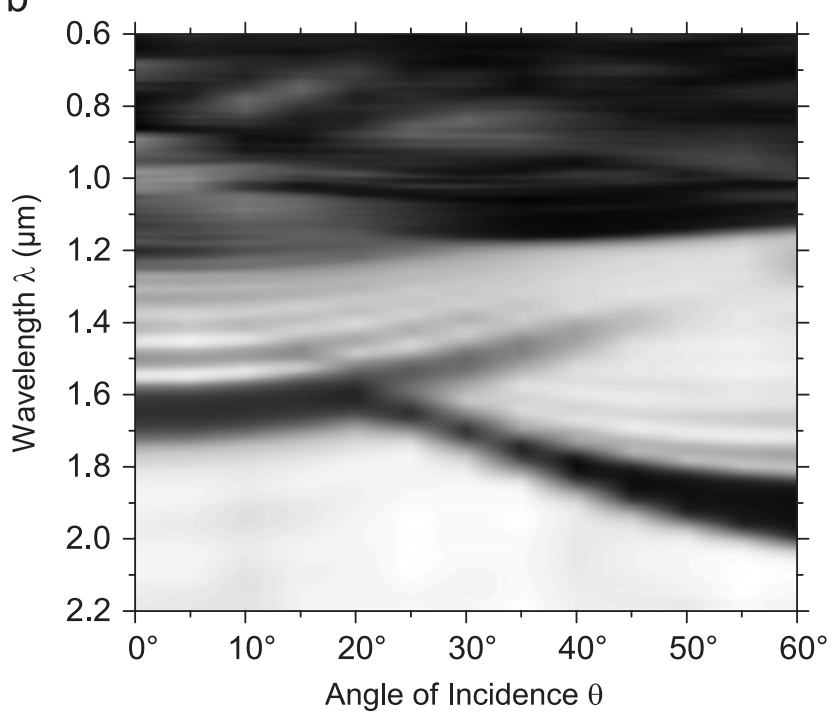

Fig. 30. Gray-scale plot of the measured transmittance versus angle of incidence and wavelength. (a) s-polarization, (b) p-polarization. Taken from [88].

How good are these photonic-crystal templates really? The optical characterization presented in Ref. [87] already suggested excellent quality, but was still obscured by a large numerical aperture of the Cassegrain measurement optics, which effectively averages over different angles of incidence, thus averaging over different directions in the reciprocal space of the photonic crystal. Using an improved measurement setup with largely reduced numerical aperture $\left(5^{\circ}\right.$ halfopening angle), yet still allowing for measuring broadband spectra on small samples [88], improved data have been obtained. In order to allow for an overview, the set of measured spectra (for individual spectra see Ref. [88]) has been converted into a gray-scale plot, where the transmittance is depicted as a function of angle of incidence with respect to the surface normal and wavelength. On the gray-scale, dark areas correspond to low transmittance, bright areas to high transmittance-allowing for an intuitive interpretation. The corresponding images in Fig. 30 reveal a rich fine structure. For normal incidence, the spectra are not quite identical because the rod direction for the uppermost layer is 
parallel (orthogonal) to the electric field in s-polarization (p-polarization). In other words: An influence of the surface termination on the optical properties is observed. Furthermore, Fabry-Perot fringes are observed even energetically above the fundamental photonic stop band. Increasing the angle of oblique incidence, the fundamental stop band shifts to the blue, hits another shifting structure, and eventually shifts to the red end of the spectrum. Note that the details of this crossing scenario are different for the two linear polarizations.

To allow for a direct comparison with the expectations for a perfect structure and to interpret them, both scatteringmatrix calculations and band structure calculations (for the parameters of the above woodpile structures) have been performed. Caution has to be taken here as we define transmittance such that it refers to the zeroth diffraction order. The higher diffracted orders (which are also calculated) must not be incorporated into the transmittance. Still, the diffracted orders take away energy from the zeroth transmitted order, hence, they reduce the transmittance. This can be a very efficient "channel" and must not be confused with scattering losses. The results of the scattering-matrix calculations (see Section 2.1.4.2) are summarized in Fig. 31, those of the band structure calculation in Fig. 32 (also see Section 2.1.4.1), where only the relevant part of the BZ is shown. Notably, the gray-scale plots of the calculated transmittance (Fig. 31) and those of the measured transmittance (Fig. 30) agree very well. This is also true for the polarization dependence. The comparison with the photonic band structure (Fig. 32) gives additional insight. Starting from the $\Gamma \mathrm{X}^{\prime}$ direction for normal incidence, the fundamental stop band shifts to the blue when going towards the $\Gamma \mathrm{U}^{\prime}$ direction for oblique incidence. At the same time, higher bands (see gray area) shift to the red and meet the fundamental stop band at the $\mathrm{U}^{\prime}$ point. Further increasing the angle, the shift directions reverse. These features are also seen in the measured (Fig. 30) and calculated (Fig. 31) transmittance spectra. Below about $1.1 \mu \mathrm{m}$ wavelength, many additional bands occur, leading to highly structured transmittance spectra. In this spectral regime, the experimental spectra are still somewhat obscured because of finite angle averaging.

Importantly, not only woodpile structures can be fabricated by DLW. For example, crystals from the slanted pore family have also been fabricated, revealing a similarly high optical quality [271]. Whether or not slanted-pore structures are equivalent to woodpiles depends on the nomenclature: If woodpiles are understood as structures with orthogonal rods only, slanted pore structures are different in that the rods enclose an arbitrary angle and because they are rotated in space. In any case, the mere rotation itself does make a difference for DLW as it somewhat reduces the undesired ellipticity of the resulting rods and because the photonic-crystal surface termination can be controlled [271]. Indeed, for the woodpiles above, we have seen (Fig. 30) that s- and p-polarization of the incident light are inequivalent, which can be interpreted as an effect of the surface termination already. Furthermore, also three-dimensional square-spiral structures (see Section 2.2.3.1) have been fabricated with (convincing) stop bands at wavelengths larger than $2 \mu \mathrm{m}$ [272]. For suitable design, circular-spiral photonic crystals made by DLW exhibit polarization stop bands for circularly polarized incident light [226,273] — which can be interpreted as giant circular dichroism without static magnetic fields. This effect can provide the basis for "thin-film" optical isolators.

The main beauty of DLW, however, is that it conceptually allows for the incorporation of functional elements (see Section 2.1.3) of arbitrary complexity. Among the most complex and demanding architectures that have been proposed theoretically are 3D-2D-3D photonic-crystal heterostructures [274], which have been predicted to be superior to 2D slab waveguide structures. An example fabricated by DLW is shown in Fig. 33 [275]. The electron micrographs reveal a three-dimensional woodpile structure underneath a two-dimensional square lattice of cylindrical posts with lines of missing posts forming a waveguide structure. The parameters are not chosen to optimize a particular performance, but rather meant to show the flexibility of DLW. Further defect structures made by DLW can be found in [272].

2.2.3.5. Silicon double inversion. We have seen that holographic lithography and direct laser writing are capable of delivering large-scale, high-quality three-dimensional nanostructures for photonics in a versatile fashion. However, to achieve three-dimensional photonic crystals with a complete band gap, the refractive index of typical photoresists, such as SU-8 $(n=1.57)$, is too small. Thus, infiltration with a high-index material is desirable. First results on the infiltration of $\mathrm{TiO}_{2}$ via CVD at room temperature have recently been presented [276]. Unfortunately, typical deposition temperatures for silicon via CVD (see Section 2.2.3.2) are not compatible with the glass temperature of the resist. The structures would melt in the reactor. To infiltrate silicon, a two-step procedure has recently been proposed and realized [277]: In a first step, $\mathrm{SiO}_{2}$ is infiltrated via ALD at room temperature (see Section 2.2.3.2), followed by removal of the SU-8 via calcination (see Fig. 34). In a second step, in perfect analogy to the infiltration of $\mathrm{SiO}_{2}$ opals addressed above, silicon is infiltrated via CVD, followed by wet chemical etching of the $\mathrm{SiO}_{2}$. Ideally, this double-inversion procedure leads to a positive silicon replica of the original SU-8 structure. Electron micrographs of corresponding structures are 


\section{a}

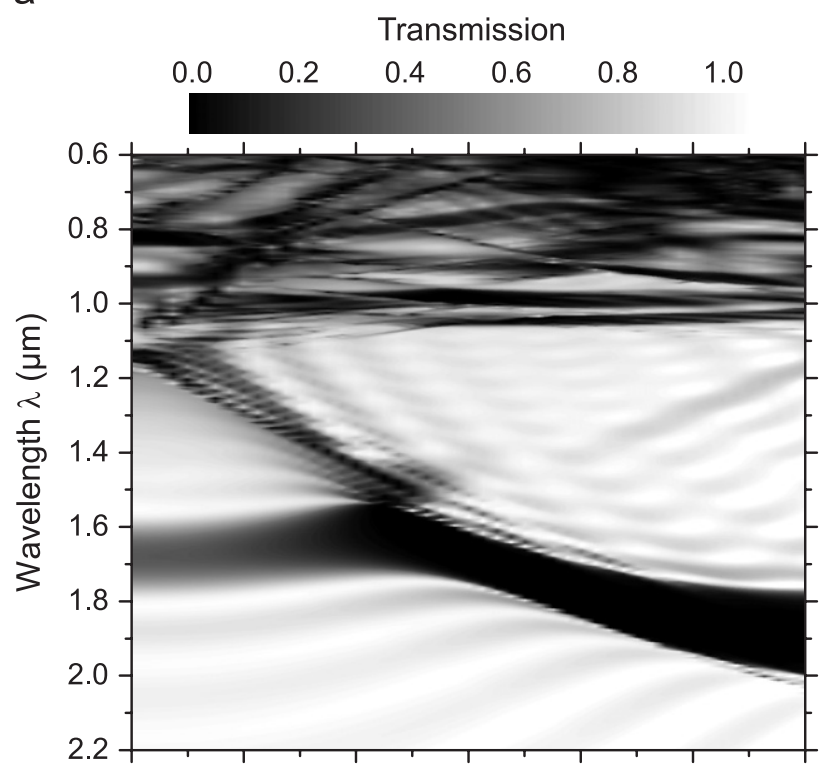

b

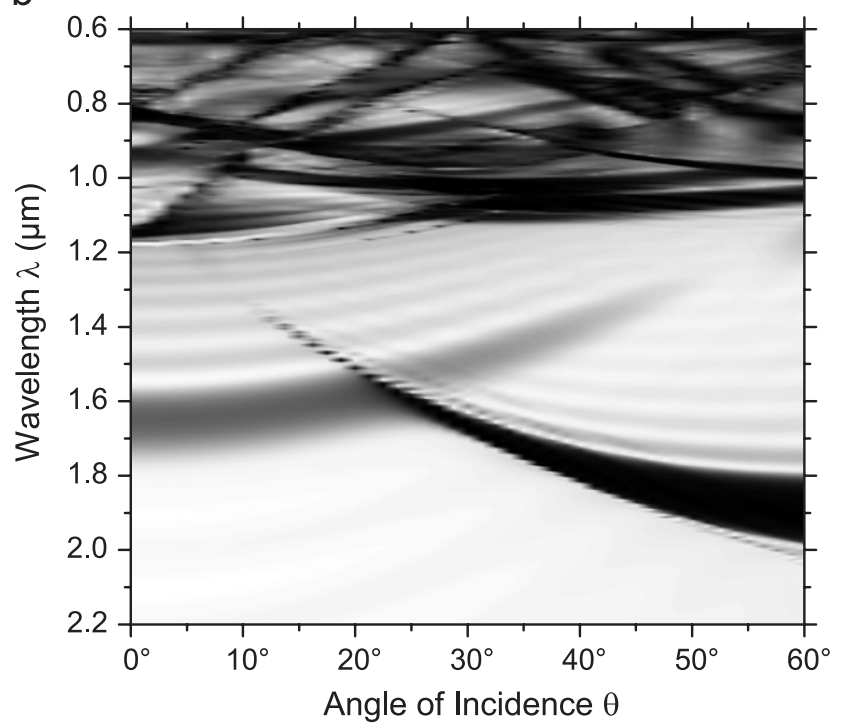

Fig. 31. Gray-scale plot of the calculated transmittance versus angle of incidence and wavelength. The woodpile structure corresponds to that of the experiment (Fig. 30). (a) s-polarization, (b) p-polarization. Taken from [88].

shown in Fig. 35. The measured optical spectra [277] of these structures (with a rod spacing of $a=0.94 \mu \mathrm{m}$ and $27 \%$ silicon volume filling fraction), together with corresponding band structure calculations, indicate a complete photonic band gap centered around $2.3 \mu \mathrm{m}$ wavelength with a gap/midgap ratio of $8.6 \%$.

2.2.3.6. Direct laser writing into high-index chalcogenide glasses. The silicon double inversion of polymeric templates described in the previous section was necessary because the three-dimensional SU-8 photoresist templates have only a small refractive index of $n=1.57$. Would not it be charming to have a resist at hand that already has a refractive index of $n=2-3$ ? In this case, no inversion or double inversion would be necessary at all. Chalcogenide glasses, such as, e.g., $\mathrm{As}_{2} \mathrm{~S}_{3}$ with $n=2.45-2.55$, are well known for their photo-induced meta-stabilities, which lead to their widespread use in 


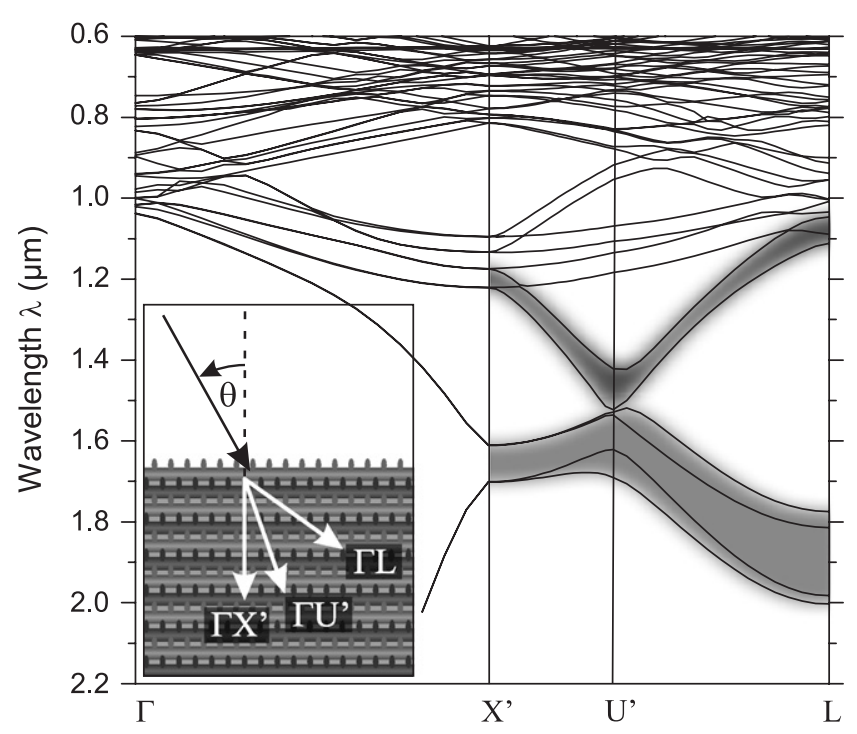

Fig. 32. Calculated band structure of the woodpile structure investigated in Figs. 30 and 31. The gray areas are guides to the eye, aiming at simplifying the comparison with Figs. 30 and 31. The inset illustrates characteristic propagation directions. Taken from [88].

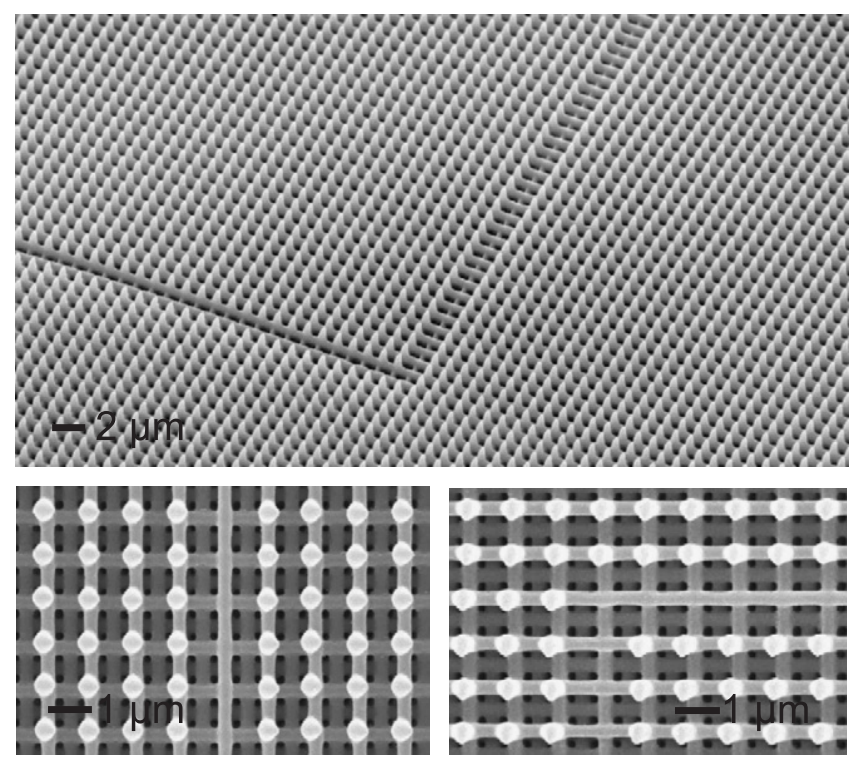

Fig. 33. Electron micrographs of a 3D-2D photonic-crystal heterostructure fabricated by direct laser writing. To allow for the view onto the $2 \mathrm{D}$ structure with embedded waveguides and bends, the top 3D photonic crystal has not been written here. It has been added in other structures not depicted here. Also see Ref. [275].

optical storage media like compact disk ROM (CD-Rs) and digital-versatile disks (DVD) [278]. Chalcogenide glasses not only change their optical properties under photo-exposure, but also their chemical properties—which makes them somewhat similar to photoresists. However, early work $[279,280]$ in this direction has not led to competitive results by today's standards. First, the chemistry is challenging. Second, the large refractive index is problematic because of refraction at the air/glass-chalcogenide interface. While compensation for refraction is possible in holographic lithography by means of immersion systems (see the discussion above), finding actual (noncancerogenic) immersion liquids with refractive indices of $n=2-3$ is not easy. In direct laser writing, the refraction at the interface towards the 


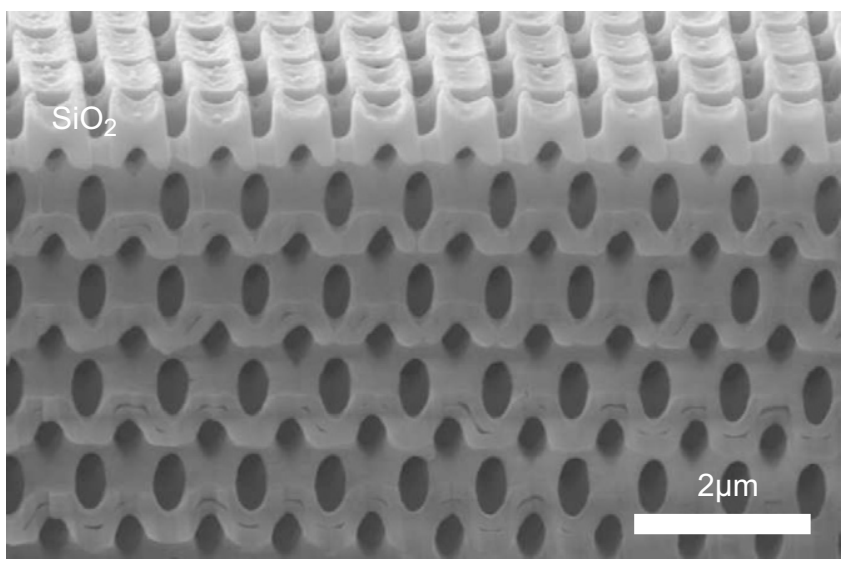

Fig. 34. Focused-ion-beam cross-section of $\mathrm{a} \mathrm{SiO}_{2}$ inverse woodpile (prior to infiltration with $\mathrm{Si}$, see Fig. 35). The structure has been fabricated via DLW and backfilling with $\mathrm{SiO}_{2}$ using atomic-layer deposition [277]. The photoresist SU-8 has been removed by chalcination. Reprinted figure with permission of M. Hermatschweiler.
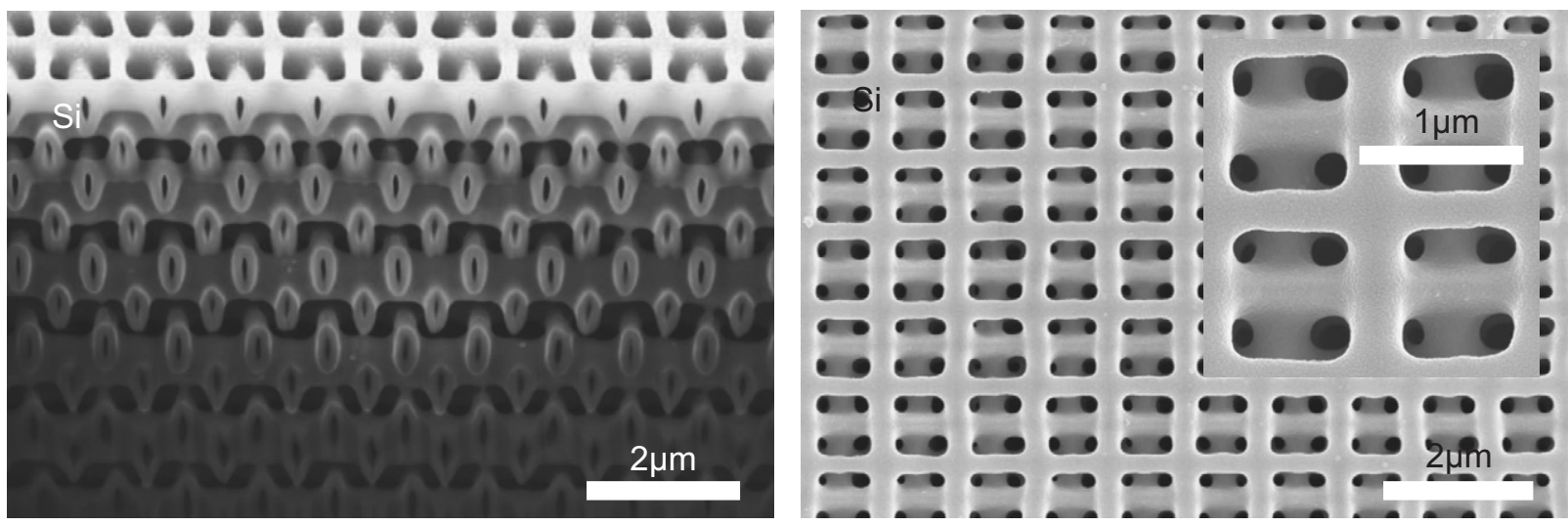

Fig. 35. (Left) Focused-ion-beam cross-section of a Si woodpile structure. The structure shown in Fig. 34 has been backfilled with Si via chemical-vapor deposition. The $\mathrm{SiO}_{2}$ has been removed by etching [277]. Note the complete and uniform infiltration with Si. (Right) Top-view electron micrograph. Note the very small residual surface roughness apparent in the inset. Reprinted figure with permission of M. Hermatschweiler.

surface normal effectively reduces the numerical aperture of the focusing optics, hence leading to largely elongated "voxels" with aspect ratios approaching 10 for an external numerical aperture of NA $=1.4$. Furthermore, the aberrations originating from the discontinuity of the refractive index lead to a "voxel" shape that strongly depends on the focusing depth. This necessitates software and/or hardware compensation approaches that are starting to emerge [281]. If these technical problems could be solved, holographic lithography and/or direct laser writing on chalcogenide glasses would be a very promising new avenue for fabricating high-index three-dimensional nanostructures for photonics.

\section{Nonlinear optics}

As compared to the linear case, nonlinear effects allow for a dynamical control of the flow of light with several potential applications in telecommunication, information processing, and sensing. Moreover, owing to the universal nature of nonlinear phenomena, nonlinear photonic crystals are of fundamental importance in their own right.

The nonlinear response of isotropic effective nondispersive and lossless dielectric materials enters Maxwell's equation through the constitutive relation [142]

$$
\vec{D}(\vec{r}, t)=\epsilon_{0} \epsilon(\vec{r}) \vec{E}(\vec{r}, t)+\vec{P}_{\mathrm{NL}}(\vec{r}, t),
$$


which is the generalization of the discussion in Section 2.1. This is justified because periodic nanostructures induce a linear dispersion that far exceeds any known material dispersion.

As usual, the nonlinear polarization $\vec{P}_{\mathrm{NL}}$ may be expanded into powers of the electric field according to

$$
\left(\vec{P}_{\mathrm{NL}}\right)_{i}=\epsilon_{0} \chi_{i j k}^{(2)}(\vec{r}) E_{j} E_{k}+\epsilon_{0} \chi_{i j k l}^{(3)}(\vec{r}) E_{j} E_{k} E_{l}+\cdots .
$$

The indices $\{i, j\}$ run over the cartesian coordinate axes $\{x, y, z\}$ labeled by $\{1,2,3\}$ and a summation over repeated indices is assumed. In the case of strictly periodic materials, the higher-order susceptibility tensors in (3.47) are periodic functions of the spatial variable $\vec{r}$. Truncating the above expansion is justified provided that the electric field amplitude is sufficiently small (3.47).

The first term on the RHS of (3.47) is responsible for second-order nonlinear effects such as second-harmonic generation. However, if waves interacting via the second-order nonlinearity $\chi_{i j k}^{(2)}$ are not phase matched or if the periodic nanostructures are fabricated from (on the microscopic level) centro-symmetric materials such as silicon, this second-order susceptibility term can be neglected.

In this case, the leading third-order nonlinearity gives rise to the optical Kerr effect, i.e., intensity-dependent refractive indices of dielectrics. Note that for isotropic materials all tensorial variables can be reduced to certain scalar functions [282].

There are two central aspects making nonlinear phenomena in periodically structured dielectrics much richer than in traditional optical systems. First, since the continuous translational invariance is broken, the wave vector is conserved only up to reciprocal lattice vectors. This greatly simplifies the phase-matching problem in the case of second-order nonlinear processes such as sum-frequency generation. Second, the carrier waves in periodic nanostructures are Bloch waves rather than plane waves. This aspect allows to tailor the mode profile as well as the linear dispersion relation, hence, the group velocity. Both aspects can lead to an enhanced nonlinear response.

\subsection{Centro-symmetric constituent materials}

We start with a discussion of one-dimensional photonic crystals composed of isotropic nonlinear Kerr-type constituent materials. Furthermore, we assume that surface effects play no role. In this case, the scalar wave equation for the electric field can readily be obtained from Maxwell's equations

$$
\left(\frac{\partial^{2}}{\partial x^{2}}-\frac{\epsilon(x)}{c_{0}^{2}} \frac{\partial^{2}}{\partial t^{2}}\right) E(x, t)=\mu_{0} \frac{\partial^{2}}{\partial t^{2}} P_{\mathrm{NL}}(x, t) .
$$

Without loss of generality, the electric field vector $\vec{E}=(0, E, 0)$ is linearly polarized perpendicular to the propagation direction. The nonlinear polarization reduces to

$$
P_{\mathrm{NL}}(x, t)=\epsilon_{0} \chi^{(3)}(x) E^{3}(x, t)
$$

The nonlinear susceptibility $\chi^{(3)}(x)$ shall have the same period $a$ as the dielectric function $\epsilon(x)=n^{2}(x)$. In the weakly nonlinear case, the RHS of (3.48) is much smaller than its LHS.

Early studies of bistability in such systems have been carried out by Winful $[283,284]$. The interest into the nonlinear dynamics of these structures experienced a significant boost when in 1987 Chen and Mills [285,286] discovered what is now known as gap solitons. Through numerical calculations these authors have found that increasing the power of radiation incident on a layered structure for frequencies in the (linear) stop gap can switch the system from a state with low transmittance to a state with near-unity transmittance. Most interestingly, it has been shown that solitary wave excitations (see Fig. 36) are responsible for this phenomenon.

To understand the physics of this unexpected and qualitatively new behavior of light in nonlinear PBG materials, we first examine the linear dispersive properties of the problem in more detail [287,288]. In the frequency domain, the linear wave equation reads

$$
\left(\frac{\mathrm{d}^{2}}{\mathrm{~d} x^{2}}+\frac{\omega^{2}}{c_{0}^{2}} n^{2}(x)\right) E(x)=0 .
$$




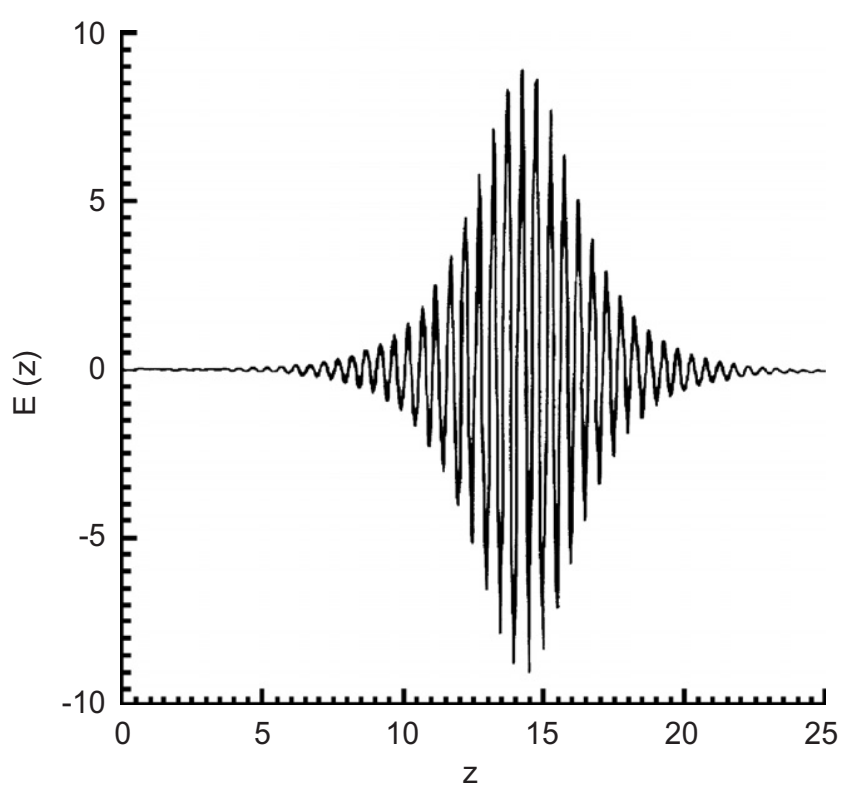

Fig. 36. Illustration of the stationary gap soliton that has been found in Ref. [285] through numerical integration of the nonlinear wave equation. Importantly, the group velocity of this soliton is strictly zero.

For the sake of simplicity, we assume a refractive index profile according to [287,288]

$$
n(x)=\bar{n}+\Delta n \cos \left(2 k_{0} x\right),
$$

where $\bar{n}$ denotes the average refractive index, and $\Delta n$ the index modulation amplitude with period $a=\pi / k_{0}$. This implies that the edges of the first BZ correspond to wave number $k= \pm k_{0}$. Eq. (3.51) is an appropriate description of fiber Bragg gratings [157].

The above model can easily be solved analytically [287]. The central frequency $\omega_{0}$ of the band gap is at

$$
\omega_{0}=\frac{k_{0} c}{\bar{n}},
$$

and its relative size is determined via

$$
\frac{\Delta \omega}{\omega_{0}}=\frac{\Delta n}{\bar{n}}
$$

Eqs. (3.52) and (3.53) suggest that—due to the dependence of $\omega_{0}$ and $\Delta \omega$ on the average refractive index $\bar{n}$ - the position of the band edge may dynamically be controlled through the optical Kerr effect. A sufficiently intense and long optical pulse will lead to an intensity-dependent refractive index

$$
n(x)=\bar{n}+\Delta n \cos \left(2 k_{0} x\right)+n_{\text {Kerr }}|E(x)|^{2},
$$

that effectively modifies the local change of the average refractive index to $\bar{n}+n_{\mathrm{Kerr}}|E(x)|^{2}$ and, consequently, will cause a local band edge shift. For $n_{\text {Kerr }}>0$, the band edges shift to lower frequencies, whereas for $n_{\text {Kerr }}<0$, they shift to higher frequencies. As a result, in Kerr-nonlinear 1D PBG materials, electromagnetic wave propagation can be realized for frequencies within the (linear) forbidden band gap.

The most general theoretical approach to the problem of weakly nonlinear wave dynamics is based on the method of multiple scales [289]. In the context of periodic optical systems, this formalism has been introduced in Refs. [290,291] and has later been extended to the case of birefringent constituent materials [292] as well as to two- and three-dimensional periodic systems [293]. 
In order to apply this technique to PBG materials, one has to explicitly introduce a small parameter $\zeta$, which defines the order of smallness of the nonlinear wave amplitude. For the electric field $E(x, t)$, one obtains [290]

$$
E(x, t) \equiv \zeta e(x, t),
$$

where $e(x, t)$ includes all perturbations which arise from weak nonlinear processes of all orders, i.e.,

$$
e(x, t)=e_{1}(x, t)+\zeta e_{2}(x, t)+\zeta^{2} e_{3}(x, t)+\cdots .
$$

At this stage in this multi-scale approach, one should formally replace the space and time variables, $x$ and $t$, with sets of independent spatial and temporal variables $\left\{x_{n} \equiv \zeta^{n} x\right\}$ and $\left\{t_{n} \equiv \zeta^{n} t\right\}$, where $n=0,1,2, \ldots[289,290]$. As a result, within the multi-scale analysis, the spatial and time derivatives have to be replaced according to

$$
\frac{\partial}{\partial x}=\frac{\partial}{\partial x_{0}}+\zeta \frac{\partial}{\partial x_{1}}+\zeta^{2} \frac{\partial}{\partial x_{2}}+\cdots
$$

and

$$
\frac{\partial}{\partial t}=\frac{\partial}{\partial t_{0}}+\zeta \frac{\partial}{\partial t_{1}}+\zeta^{2} \frac{\partial}{\partial t_{2}}+\cdots
$$

from which analogous replacements for higher-order derivatives can be constructed. Specifically, the $e_{i}$ functions are assumed to depend on all $x_{n}$ and all $t_{n}$, i.e., $e_{i}(x) \equiv e_{i}\left(\left\{x_{n}\right\},\left\{t_{n}\right\}\right)$, while the photonic-crystal parameters are functions of the smallest length scale $x_{0}$ only, i.e., $\epsilon(x) \equiv \epsilon\left(x_{0}\right)$ and $\chi^{(3)}(x) \equiv \chi^{(3)}\left(x_{0}\right)$.

Inserting (3.55)-(3.58) into (3.48) and collecting terms with equal powers in the small parameter $\xi$ facilitates the separation of the different time and length scales in the physical system into an infinite hierarchy of equations. In particular, the smallest spatial scale, $x_{0}$, corresponds to the wavelength of the carrier wave. The fastest temporal variable, $t_{0}$, is associated with the optical period. Similarly, slow spatial and time variables describe the dynamics of the envelope function of an electromagnetic pulse. This hierarchy of equations has to be truncated at the desired order of perturbation theory. Finally, in order to express the results in terms of the original physical variables, at the end of calculations one has to set $\zeta=1$.

As is shown in Ref. [290], in the moving reference frame $\eta \equiv x-v_{\text {group }} t$, the third-order multiple-scale analysis for a pulse with carrier wave $\varphi_{m}\left(x_{0}\right)$ propagating in a Kerr-nonlinear photonic crystal leads to the nonlinear Schrödinger equation (NLSE) for the corresponding envelope function $A(\eta, t)$

$$
\mathrm{i} \frac{\partial A}{\partial t}+\frac{1}{2} \omega_{m}^{\prime \prime} \frac{\partial^{2} A}{\partial \eta^{2}}+\chi_{\text {eff }}^{(3)}|A|^{2} A=0 .
$$

In this expression, the carrier wave $\varphi_{m}\left(x_{0}\right)$ represents a Bloch function at frequency $\omega_{m}$ and the corresponding group velocity $v_{\text {group }}=\partial \omega_{m} / \partial k$ as well as the group velocity dispersion $\omega_{m}^{\prime \prime}=\partial^{2} \omega_{m} / \partial k^{2}$ can be calculated using $\vec{k} \cdot \vec{p}$ perturbation theory (see Section 2.1.1.3). Furthermore, the carrier wave determines the effective nonlinearity $\chi_{\mathrm{eff}}^{(3)}$ that measures how the Bloch function samples the distribution of nonlinear material within the photonic crystal's unit cell

$$
\chi_{\mathrm{eff}}^{(3)}=\frac{3 \omega_{m}}{2} \int \chi^{(3)}\left(x_{0}\right)\left|\varphi_{m}\left(x_{0}\right)\right|^{4} \mathrm{~d} x_{0} .
$$

Eq. (3.59) demonstrates that in the weakly nonlinear limit, the nonlinear response of a photonic crystal is equivalent to the response of a homogeneous material with effective parameters $v_{\text {group }}, \omega_{m}^{\prime \prime}$, and $\chi_{\mathrm{eff}}^{(3)}$. This manifests the fact that the envelope of a long pulse varies much slower in space than the spatial period of the PBG material (see Fig. 36). Moreover, from (3.60) one infers that the effective nonlinearity in a PBG material may be enhanced or suppressed relative to the nonlinear response of a homogeneous material. This is due to the fact that the Bloch functions do not uniformly distribute the electric field amplitude between the constituent materials of the photonic crystal [294]. Indeed, as demonstrated experimentally in Ref. [295], the effective third-order susceptibility of a composite dielectric can exceed that of the constituent materials.

The NLSE (3.59) describes the dynamics of weakly nonlinear waves in 1D Kerr-nonlinear PBG materials. This equation exhibits soliton solutions when the Lighthill condition, $\chi_{\mathrm{eff}}^{(3)} \omega_{m}^{\prime \prime}>0$, holds [289]. To distinguish between 
different physical situations, which appear for carrier wave frequencies within and outside the band gaps, respectively, two types of solitons may be introduced. When the carrier frequency of a soliton is near, but outside, the band gap and the localized pulse propagates with a velocity much less than the speed of light $c_{0} / \bar{n}$, the corresponding soliton is called a Bragg soliton [288]. Similarly, if the central frequency of a nonlinear localized pulse lies within a photonic band gap, it is called a gap soliton.

Gap solitons can exhibit vanishing propagation velocity [285,286,288,290] (see Fig. 36). Clearly, when the group velocity $v_{\text {group }}$ is zero, no energy is being transported and the carrier wave of the pulse is a standing wave, i.e., a Bloch function at the band edge. Such nonlinear pulses with zero group velocity correspond to stationary solutions of (3.59).

The multiple-scales analysis allows to study the nonresonant wave interaction processes within the NLSE model [296,297]. In this regime, two interacting solitons have sufficiently different group velocities to pass through each other quickly enough to avoid any resonant effects such as energy exchange. More precisely, the larger the spectral overlap between the interacting solitons becomes, the larger the relative velocity between them should be in order to realize the nonresonant regime. Then, and in contrast to the resonant regime, the interacting solitons obey two different NLSEs with different coefficients. Therefore, only in this nonresonant regime can the richness of the dispersive properties of PBG materials be fully exploited.

Owing to the integrability of the NLSE model, the effect of such nonresonant interactions manifests itself in an overall phase-shift of the carrier waves which directly translates into a shift of the wave front. The overall wavefront shift, $\Delta l_{1}$, of a wave with envelope $A_{1}$ caused by the interaction with a wave with envelope $A_{2}$ can then be determined as

$$
\Delta l_{1}=\frac{\partial}{\partial k_{1}}\left[\frac{\Delta_{\text {eff } 1}^{(3)}}{\left(v_{\text {group }, 2}-v_{\text {group }, 1}\right)}\right] \int_{-\infty}^{+\infty} \mathrm{d} \eta_{2}\left|A_{2}\right|^{2} .
$$

Here, $k_{1}$ is the wave number of the first wave (with envelope $A_{1}$ ) and $\Delta_{\text {eff } 1}$ denotes the effective cross-phase modulation constant

$$
\Delta_{\text {eff } 1}^{(3)}=3 \omega_{m_{1}} \int \mathrm{d} x_{0} \chi^{(3)}\left(x_{0}\right)\left|\varphi_{m_{1}}\left(x_{0}\right)\right|^{2}\left|\varphi_{m_{2}}\left(x_{0}\right)\right|^{2}
$$

Eq. (3.61) suggests that one may control the position of one nonlinear pulse (pulse one) via the duration and intensity of a colliding nonlinear wave (pulse two). In fact, in the range of parameters where the NLSE is a valid model, there is excellent agreement between the analytical results of (3.61) and direct numerical calculations [296,297]. Nonresonant interaction processes between Bragg and stationary gap solitons may be of particular importance: The moving Bragg solitons can be used to control and manipulate stationary gap solitons. Conversely, the wave front shift experienced by the Bragg solitons provides a route to probe the existence and properties of the stationary gap soliton. Therefore, the wave-front shifts associated with nonresonant interaction processes of solitons in PBG materials could have potential for applications such as all-optical buffers and logic gates. In addition, we would like to note that (3.61) is valid for all pulses that are well described by the NLSE and does not depend on the details of the particular system's governing equation. Therefore, similar effects as those discussed above may, for instance, be realized in Bose-Einstein condensates in optical lattices, where recently gap solitons have been demonstrated experimentally [298].

However, we would like to emphasize that the above statements are valid in the integrable NLSE limit only. In general, when the equations of motion that govern the nonlinear dynamics are nonintegrable, inelastic effects are present during the interaction processes.

In particular, when the frequency of electromagnetic radiation is lying deep within the PBG, the NLSE fails to be a valid model [288]. In this case, the nonlinearity must be sufficiently strong in order to tune the band edge and to allow light propagation in the sample. As a result, the system must be described via the so-called nonlinear coupled mode equations (NLCME). In its simplest form, the NLCME considers a forward and backward propagating plane wave which are coupled through the Bragg scattering in a PBG material. Thus, the starting point of the derivation of NLCME is (3.48), where a weak modulation of the refractive index $n(x)$ is assumed so that two plane waves provide a sufficiently accurate description of the linear properties near the corresponding Bragg resonance [288]. Specifically, we assume $\Delta n \ll \bar{n}$ in (3.51). 
The central idea of the coupled-mode approach is to search for solutions of the wave equation (3.48) of the form

$$
E(x, t)=E_{+}(x, t) \exp \left[-\mathrm{i}\left(\omega_{0} t-k_{0} x\right)\right]+E_{-}(x, t) \exp \left[-\mathrm{i}\left(\omega_{0} t+k_{0} x\right)\right]+\text { c.c. }
$$

where, in the case of a nonvanishing modulation $\Delta n \neq 0$, the forward $E_{+}(x, t)$ and backward $E_{-}(x, t)$, wave amplitudes must be regarded as being nonzero even in the case of an infinite sample. In other words, to lowest order, a weak modulation $\Delta n \ll \bar{n}$ in the dielectric profile couples forward and backward propagating plane waves. However, for the case of strong periodic modulations of the refractive index, the carrier waves associated with a nonlinear pulse are Bloch functions and any approximation based on a few plane waves is invalid. Instead, a multiple-scales approach with at least two equally important carrier waves, one forward and one backward propagating, must be employed [299,300]. However, in order to avoid additional notational complexity, we will refrain from elaborating on this issue and refer to Refs. [299,300] for details.

Inserting (3.63) into (3.48) and using the slowly varying envelope approximation one obtains the NLCME

$$
\begin{aligned}
& \mathrm{i} \frac{\bar{n}}{c_{0}} \frac{\partial E_{+}}{\partial t}+\mathrm{i} \frac{\partial E_{+}}{\partial x}+\kappa E_{-}+\Gamma\left(\left|E_{+}\right|^{2}+2\left|E_{-}\right|^{2}\right) E_{+}=0, \\
& \mathrm{i} \frac{\bar{n}}{c_{0}} \frac{\partial E_{-}}{\partial t}-\mathrm{i} \frac{\partial E_{-}}{\partial x}+\kappa E_{+}+\Gamma\left(\left|E_{-}\right|^{2}+2\left|E_{+}\right|^{2}\right) E_{-}=0,
\end{aligned}
$$

where the linear coupling constant $\kappa$ and the nonlinear coefficient $\Gamma$ are

$$
\begin{aligned}
\kappa & =\frac{\Delta n}{2 \bar{n}} k_{0}, \\
\Gamma & =\frac{6 \pi}{\bar{n}^{2}} \chi^{(3)} k_{0} .
\end{aligned}
$$

In deriving the NLCME (3.64), "nonphase-matched" terms that involve exp $\left(+2 \mathrm{i} k_{0} x\right)$ have an amplitude of order $\kappa^{2}$ and, therefore, have been neglected [288]. This is only justified if $\Delta n \ll \bar{n}$.

The use of the NLCME instead of the NLSE model comes with certain advantages as well as certain disadvantages. The main advantage is that within the NLCME formalism one can describe much shorter pulses as compared to the NLSE model. This is a crucial generalization, especially for the description of nonlinear wave dynamics in the frequency range deep within the band gaps. More specifically, for sufficiently long, i.e., spectrally sufficiently narrow, pulses near the band edge, the NLCME can be reduced to the NLSE [301,288]. Furthermore, the NLCME allows one to take into account the exact dispersion of a PBG material. Unfortunately, the price for these desirable properties is that the NLCME are nonintegrable and, consequently, it is much more difficult to obtain analytical results within the NLCME model. Nevertheless, several authors [302,303] have been able to derive solitary wave solutions of the form

$$
E_{ \pm}(x, t)=\alpha \tilde{E}(x, t) \exp (\mathrm{i} \xi(\theta)),
$$

where $\tilde{E}_{ \pm}$denote the well-known solutions of the (integrable) massive Thirring model [304]

$$
\begin{aligned}
& \tilde{E}_{+}= \pm \sqrt{ \pm \frac{\kappa}{2 \Gamma}} \frac{1}{\Delta} \frac{\sin \hat{\delta}}{\cosh (\theta \mp \mathrm{i} \hat{\delta} / 2)} \exp ( \pm \mathrm{i} \beta), \\
& \tilde{E}_{-}=-\sqrt{ \pm \frac{\kappa}{2 \Gamma}} \frac{1}{\Delta} \frac{\sin \hat{\delta}}{\cosh (\theta \pm \mathrm{i} \hat{\delta} / 2))} \exp ( \pm \mathrm{i} \beta) .
\end{aligned}
$$

In (3.67), the choice of signs has to guarantee that the radicals are positive. Obviously, this choice is determined by the relative sign of the linear and nonlinear coupling coefficients. Furthermore, the quantities $\theta$ and $\beta$ are defined as

$$
\begin{aligned}
& \theta=\kappa\left[x-\frac{c_{0}}{\bar{n}} v t\right] \frac{\sin \hat{\delta}}{\sqrt{1-v^{2}}}, \\
& \beta=\kappa\left[v x-\frac{c_{0}}{\bar{n}} t\right] \frac{\cos \hat{\delta}}{\sqrt{1-v^{2}}},
\end{aligned}
$$


where the scaled soliton velocity $v$ (see below) is

$$
v=\frac{1-\Delta^{4}}{1+\Delta^{4}}
$$

Here, $\Delta$ (or, equivalently $v$ ) is a free parameter which may take on any real value (or, equivalently, any value $|v|<1$ ). Finally, the expressions for $\alpha$ and the phase function $\xi(\theta)$ are

$$
\begin{aligned}
& \frac{1}{\alpha^{2}}=1+\frac{1}{2} \frac{1+v^{2}}{1-v^{2}}, \\
& \exp (\xi(\theta))=\left[-\frac{\exp (2 \theta)+\exp (\mp \mathrm{i} \hat{\delta})}{\exp (2 \theta)+\exp ( \pm \mathrm{i} \hat{\delta})}\right]^{W},
\end{aligned}
$$

where we have introduced the dimensionless quantity $W=2 v /\left(2\left(1-v^{2}\right)+\left(1+v^{2}\right)\right)$.

Note that the family of solitary wave solutions (3.67) is completely characterized by two independent parameters $\hat{\delta}$ and $v$, where $|v|<1$ and $0 \leqslant \hat{\delta} \leqslant \pi$. An inspection of (3.69) and (3.70) confirms our above assertion that $v$ parametrizes the soliton velocity. It can take on any value between 0 and \pm 1 which is to say that the solitary wave can travel with any velocity between zero and the speed of light $c_{0} / \bar{n}$ in the medium. In addition, (3.68)-(3.70) demonstrate that $\hat{\delta}$ simultaneously determines the soliton's width, height, and spectrum. This implies that $\hat{\delta}$ parametrizes the detuning of the solitary wave from the Bragg frequency. In particular, it is straightforward to verify from (3.63) and (3.70) that in the limit $\hat{\delta} \rightarrow 0$ and if the nonlinearity is positive (negative), the central frequency $\Omega_{\mathrm{sw}}$ of the solitary wave (see (3.70))

$$
\Omega_{\mathrm{sw}}=\frac{c}{\bar{n}} \frac{\kappa \cos \hat{\delta}}{\sqrt{1-v^{2}}}
$$

is tuned to the upper (lower) band edge of the photonic band gap. In fact, this situation corresponds to the NLSE limit of the NLCME. In contrast, the limit $\hat{\delta} \rightarrow \pi$ implies that $\Omega_{\mathrm{sw}}$ is tuned to the lower (upper) band edge. For $\hat{\delta}=\pi / 2$, the central frequency of the solitary wave is exactly in the middle of the gap. Moreover, $\Omega_{\mathrm{sw}}$ lies in the (for linear wave propagation) forbidden band gap if [288]

$$
|v|<\sin \hat{\delta} .
$$

In this case, the solitary wave is referred to as a gap soliton. It is a rather noteworthy fact, that one can find a parameter range for which the entire frequency content of the solitary waves lies within the band gap

$$
v<\sin \hat{\delta}<\frac{\sqrt{1-v^{2}}}{v} .
$$

In the opposite case, when $|v|>\sin \hat{\delta}$, the solitary wave's central frequency is outside the band gap and, therefore, corresponds to a Bragg soliton. Strictly speaking, the term soliton should only be used for those localized solutions of the (nonintegrable) NLCME that fall in the (integrable) NLSE limit. However, very often this rigor is relaxed and all solitary wave solutions of the NLCME are referred to as solitons.

The nonintegrability of the NLCME leads to the existence of internal vibrational modes of the solitary solutions. These vibrational modes may have a profound impact on the solitary-wave dynamics. In fact, it has been shown numerically and analytically [305-307] that for higher intensity solitons, oscillatory instabilities occur which eventually lead to the destruction of the localized waves. A corresponding stability analysis for the stationary solutions of the NLCME has been reported in Ref. [308].

Moreover, it is well known that the internal modes play an important role in complex interaction processes of solitary waves with defects and other waves [309]. This generic scenario has been confirmed in recent studies of the NLCME. For instance, in Ref. [310], the interaction of gap solitons with localized defects, that support linear defect modes, has been investigated and parameter regimes for the capture at, reflection from, and transmission through defect sites have been identified. In addition, it has been realized that the aforementioned three regimes of soliton-defect interaction may even occur when the gap solitons interact with delta-like defects, which themselves do not exhibit linear defect modes [311] 
and pairs of such defects are capable of trapping gap solitons [312]. In addition, an analysis of solitary wave interacting with a defect that exhibits gain in an overall lossy Bragg grating has been presented in Ref. [313]. Furthermore, an effective-particle approach for describing the interaction between solitary waves with localized and extended defects within Bragg gratings has been developed in Refs. [314,315], thereby extending earlier studies [316,317] of the effects of gain, loss, and two-photon absorption on solitons in fiber Bragg gratings. Finally, in Ref. [318], numerical studies of inelastic effects during collision processes of gap solitons have been carried out. In particular, these calculations demonstrate that two moving solitary waves may merge into a single stationary pulse.

Solitary waves in two- and three-dimensional PBG materials have been analyzed in Ref. [319]. In this work, the nonlinear dynamics has been reduced to an effective Dirac-type equation and resulting solitary wave solutions carry information about the underlying lattice symmetries.

Experimentally, Bragg solitons have been observed in optical fiber Bragg gratings [320] and very good agreement between theory and experiment has been found. Numerical studies of gap-soliton generation and switching in finite periodically modulated dielectrics is presented in Refs. [321-323] and nonlinear self-switching in periodic PBG materials has been demonstrated experimentally [324,325].

To date, the unambiguous experimental demonstration of launching or creating stationary gap solitons remains an unresolved and highly interesting issue the value of which would go well beyond basic nonlinear physics. In fact, gap solitons show great promise for the realization of all-optical buffers and storage devices [326] and more advanced applications may include fiber-optical logic gates and all-optical computation [157].

The Kerr effects discussed so far dominate the nonlinear effects in structured dielectrics as long as the resonant interaction of the constitutive material's atoms with the optical wave field is either absent or negligible. If this condition is violated, one needs to resort to the optical Maxwell-Bloch equations for modeling the dynamics [289]. For instance, for a (one-dimensional) periodic sequence of thin layers each consisting of two-level atoms this reads as [327]

$$
\begin{aligned}
& \frac{\partial^{2} \mathscr{E}}{\partial t^{2}}-\frac{\partial^{2} \mathscr{E}}{\partial x^{2}}=2 \int_{-\infty}^{\infty} \frac{\partial P}{\partial t} g(\Delta \omega) \mathrm{d} \Delta \omega, \\
& \frac{\partial P}{\partial t}=\mathscr{N} \mathscr{E}-\mathrm{i} \tau_{\mathrm{c}} \Delta \omega P \\
& \frac{\partial \mathscr{N}}{\partial t}=-\frac{1}{2}\left(P^{*} \mathscr{E}+P \mathscr{E}^{*}\right)
\end{aligned}
$$

and

$$
\frac{\partial^{2} \tilde{\mathscr{E}}}{\partial t^{2}}-\frac{\partial^{2} \tilde{\mathscr{E}}}{\partial x^{2}}=-2 \int_{-\infty}^{\infty} \frac{\partial P}{\partial x} g(\Delta \omega) \mathrm{d} \Delta \omega .
$$

In these equations, the atom's inhomogeneously broadened linewidth is accounted for through the function $g(\Delta \omega)$, which is assumed to be sufficiently narrow to fit into the band gap $\left(g(\Delta \omega)=\delta\left(\omega-\omega_{0}\right)\right.$ for the case of homogeneous broadening). In (3.77), we have introduced $\mathscr{E}=\mathscr{E}_{+}+\mathscr{E}_{-}$and $\mathscr{E}=\mathscr{E}_{+}-\mathscr{E}_{-} . \mathscr{E}_{ \pm}=2 \tau_{\mathrm{c}}\left(d_{2 \mathrm{LS}} / \hbar\right) E_{ \pm}$are the normalized forward and backward propagating waves. In addition, we have defined $P=P^{\prime} \exp (-\mathrm{i} \Delta \omega t)$, where $P^{\prime}$ denotes the dimensionless atomic polarization. $\tau_{\mathrm{c}}$ stands for the cooperative time, which is a measure of the photon lifetime in the medium [327] and $\Delta \omega=\omega-\omega_{0}$ defines the detuning of the light frequency from the atom resonance frequency. Finally, $d_{2 \mathrm{LS}}$ is the two-level system dipole matrix element and $t=t^{\prime} / \tau_{\mathrm{c}}$ and $x=x^{\prime} / c_{0} \tau_{\mathrm{c}}$ are dimensionless time and space coordinates. Here, we have tacitly assumed that the lattice periodicity equals the wavelength of the incident optical pulse (Bragg condition).

In Ref. [327], the exact gap soliton solution of (3.77) has been found. In the limit of homogeneous line broadening and zero detuning $(\Delta \omega=0)$, numerical and analytical studies demonstrate the existence of oscillating solitary wave solutions, so-called zoomerons [328].

In the further limiting case that the resonant layer is much thinner than the wavelength, an entire family of solitary wave solutions is obtained via analytical calculations [329,330]. Moreover, Ref. [331] has been shown that the system simultaneously supports bright and dark soliton solutions for the same parameters values. Later, the existence of solitary waves for two- and three-dimensional resonant systems has been demonstrated [332]. For combined Kerr-nonlinear periodic systems, uniformly doped with resonant two-level atoms, solitary wave excitations have been found and studied $[333,334]$. 
Based on these studies, resonant gap soliton interaction processes with a localized defect and/or with other resonant solitons have been investigated in a series of works [335-337]. As demonstrated in Ref. [335], such resonant solitons can be trapped, reflected from, or tunnel through defect states. In addition, trapped or stored pulses can be released through collisions with other solitons [336,337] which could lead to applications in optical switching, signal processing and quantum computing.

\subsection{Noncentro-symmetric constituent materials}

Without inversion symmetry of the constituent materials, second-order nonlinear processes come into play. The same holds if surface effects become important (surface $\chi^{(2)}$ ). The latter can also occur if the constituent materials themselves are centro-symmetric. In these cases, three-wave interactions play a dominant role provided that energy and wave-vector conservation are met, i.e.,

$$
\begin{aligned}
& \omega_{1}+\omega_{2}=\omega_{3}, \\
& \vec{k}_{1}+\vec{k}_{2}=\vec{k}_{3}+\vec{G} .
\end{aligned}
$$

Here, $\omega_{i}$ and $\vec{k}_{i}(i=1,2,3)$, respectively, are the frequencies and wave vectors of the interacting waves. In addition, $\vec{G}$ is a photonic-crystal reciprocal lattice vector (see Section 2.1.1.1). For the degenerate case of $\omega_{1}=\omega_{2}$, three-wave mixing reduces to second-harmonic generation. A process involving a triad of waves that satisfy (3.78) and (3.79) is called phase matched.

As compared to homogeneous materials, the photonic-crystal reciprocal lattice vector $\vec{G}$ in (3.79) introduces additional freedom for achieving phase matching in weakly modulated periodic system. For strong index contrast variations, the multi-branch dispersion relation of the photonic crystal, i.e., $\vec{k}\left(\omega_{i}\right)=\vec{k}_{i}(i=1,2,3)$ gives yet additional freedom. This aspect has first been pointed out for 1D systems by Bloembergen [338] and has been realized experimentally soon thereafter [339]. Note, that this process is generally distinct from quasiphase-matching [340], where the nonlinear susceptibility is spatially modulated. In homogeneous materials, phase matching can be achieved by exploiting birefringence [282]. As discussed in Section 2.1.1.1, photonic crystals in the long-wavelength limit allow for effective birefringence from isotropic material (form birefringence) [341,342].

For the special but practically important case of second-harmonic generation in one dimension, coupled mode equations can be derived [343-345] in analogy to the case of Kerr nonlinearities

$$
\begin{aligned}
& \mathrm{i}\left(\frac{\partial}{\partial t} \pm \frac{\partial}{\partial x}\right) E_{1 \pm}+\kappa_{1} E_{1 \mp}+\Gamma\left(E_{1 \pm}\right)^{*} E_{2 \pm}=0, \\
& \mathrm{i}\left(\frac{\partial}{\partial t} \pm \vartheta \frac{\partial}{\partial x}\right) E_{2 \pm}+\kappa_{2} E_{2 \mp}+\delta k E_{2 \pm}+\Gamma\left(E_{1 \pm}\right)^{2}=0 .
\end{aligned}
$$

Here, $E_{1 \pm}$ and $E_{2 \pm}$ are the slowly varying envelopes of forward and backward propagating waves at the fundamental $\omega_{1}=\omega\left(k_{1}\right)$ and the second harmonic $\omega_{2}=\omega\left(k_{2}\right)=2 \omega_{1}$ frequencies and $\Delta k=k_{2}-2 k_{1}$ represents the phase mismatch of these waves. The (linear) coupling coefficients at the two frequencies are denoted by $\kappa_{1,2}=k_{1,2} \Delta n_{1,2} / 2$, and the nonlinear coefficient is obtained as $\Gamma=\chi^{(2)} k_{1} / \bar{n}_{1}^{2}$ [344]. Furthermore, the group velocities of the fundamental and the second-harmonic waves are normalized to 1 and $\vartheta$, respectively. The theory of modulational instability in (3.80), which has important implications for the corresponding solitary wave dynamics, has been developed in Ref. [346].

Assuming that both the fundamental and the second-harmonic waves are in resonance with the Bragg grating structure, the solitary wave solutions of the model defined by (3.80) have been obtained in Ref. [344] for the case of $\vartheta=1$. There, these solutions have been extended to two and three dimensions and their stability has been verified. If both fields are tuned near the band edges, solitary wave solutions have been investigated [347] and a corresponding Bloch-function approach for strong index modulations has been presented [348]. Later, a more general class of solutions has been reported [349] and the problem of the excitation of stationary parametric gap solitons has been addressed [350]. In particular, it has been shown that stationary pulses can be formed when two counter-propagating low-velocity solitary waves collide.

If only the second-harmonic field satisfies the Bragg resonance condition, solitary wave solutions have been considered [345] and good agreement between analytical and numerical results has been reported. More recently, double-peak solitary wave solutions have been found and their stability has been studied [351]. Certain parameter regions of stable 
solitary wave solutions have been identified but numerical calculations show that the interaction processes between moving solitary waves are always destructive. In the opposite limit, when only the fundamental wave is in resonance with the periodic medium, solitary wave solutions have been found as well [352] and it has been demonstrated that under specific conditions such slow solitary waves relax to the zero-velocity stationary pulses [353].

For large phase mismatch $\Delta k$, the effective nonlinear response of a periodic system with second-order nonlinearities is, in fact, cubic (Kerr-like) rather than quadratic [354]. This result has later been corrected [345]. The corresponding Kerrlike solitons and their stability properties have been studied both, analytically and numerically $[355,356]$. Furthermore, in the case of large phase mismatch, the interaction between two perpendicularly polarized fundamental fields at the same fundamental frequency may lead to the formation of Thirring solitons $[357,288]$.

As alluded to above, in the case of strong dielectric modulation, the coupled-mode equations (3.80) must be modified. Consequently, the method of multiple scales has been employed [358] to derive a theory for strong dielectric constant variations. Subsequently, stationary bright solitary-wave solutions of this generalized theory have been obtained [359].

In contrast to the case of Kerr nonlinearities, interesting second-order nonlinear effects in photonic crystals are not necessarily associated with pulses whose frequency content has a significant component within or in close proximity to the (linear) band gaps [360]. In particular, using a multiple-scales analysis, it has been demonstrated that in twodimensional photonic crystals, $E$-polarized electromagnetic waves obey the following coupled nonlinear equations for (properly rescaled) the slowly varying envelope functions $A_{i}(i=1,2,3)$

$$
\begin{aligned}
& \left(\frac{\partial}{\partial t}+\vec{v}_{\text {group }, 1} \frac{\partial}{\partial \vec{r}}\right) A_{1}=\chi_{\text {eff }}^{(2)} A_{2}^{*} A_{3}, \\
& \left(\frac{\partial}{\partial t}+\vec{v}_{\text {group }, 2} \frac{\partial}{\partial \vec{r}}\right) A_{2}=\chi_{\text {eff }}^{(2)} A_{1}^{*} A_{3}, \\
& \left(\frac{\partial}{\partial t}+\vec{v}_{\text {group }, 3} \frac{\partial}{\partial \vec{r}}\right) A_{3}=\chi_{\text {eff }}^{(2) *} A_{1} A_{2} .
\end{aligned}
$$

Here, $\vec{v}_{\text {group }, i}$ denote the group velocities of the interacting waves and the effective second-order nonlinear coefficient $\chi_{\mathrm{eff}}^{(2)}$ is given by

$$
\chi_{\mathrm{eff}}^{(2)}=\int_{\mathrm{wsc}} \mathrm{d}^{2} r \chi^{(2)}(\vec{r}) \varphi_{m_{1}}^{*}(\vec{r}) \varphi_{m_{2}}^{*}(\vec{r}) \varphi_{m_{3}}(\vec{r})
$$

This coefficient can be chosen to be real through an appropriate phase transformation (see Section 2.1.1.1) of the Bloch functions $\varphi_{m_{i}}^{*}(\vec{r})$ that serve as carrier waves. This effective second-order nonlinearity describes how the carrier waves sample the nonlinear material distribution within a Wigner-Seitz cell of the photonic crystal and-owing to the complex symmetry properties of the Bloch functions (see Section 2.1.1.1) - are subject to certain selection rules. In the regime described by (3.81)-(3.83), photonic crystals with a second-order nonlinear response allow to realize wavefront reversals (or phase conjugations) as well as inversions of the time profile of an optical pulse [360]. In addition, a somewhat complementary nonlinear regime occurs when two pulses that are close in frequency and wave vector interact (nearly degenerate regime). In this case, the corresponding dynamics can be described through a generalized NLSE equation [360] where a third, very low-frequency wave mediates the interaction between the two high-frequency waves. In nonlinear physics such phenomena are known as long-wave short-wave interaction processes [289].

Finally, we would like to note that nonlinear photonic crystals might be utilized as very efficient sources for generating entangled photons [361,362]. Indeed, the flexibility of designing structures with very low group velocities may lead to strongly enhanced second-harmonic generation as has been suggested theoretically [363,364]. For 1D systems, this has been demonstrated experimentally [364-367].

For centro-symmetric constituent materials, phase-matched direct third-harmonic generation (THG) has been reported for 3D polymer-based opal structures [368] and for 1D liquid-crystal structures [369]. Furthermore, phasematched nondegenerate four-wave maxing has been reported for 1D chalcogenide-based photonic crystals [370]. 


\section{Quantum optics}

Photonic crystals provide a strongly modified electromagnetic environment to optically active materials embedded in them. As a result, significant control over the radiative properties of these materials may be exerted and novel phenomena may arise. In fact, one of the seminal papers on photonic crystals [5] has been concerned with the complete suppression of spontaneous emission for frequencies inside a complete 3D photonic band gap and the corresponding implications for the creation of low-threshold lasers.

Broadly speaking, quantum optics in photonic crystals may be divided into two-not entirely disconnectedcategories. On the one hand, high- $Q$ cavities in photonic crystals (see Section 2.2.2) allow to realize the so-called strong coupling regime well-known from cavity quantum electrodynamics (cavity-QED) where a single-cavity mode strongly interacts with the emitters. The novel element brought about by photonic crystals in this category is that they allow for cavities with simultaneously very high-quality factors $Q$ and very small modal volumes $V$ in solid-state-based systems. Since many effects in cavity-QED such as the Purcell effect are driven by large values of $Q / V$, photoniccrystal-based quantum optics offers unique possibilities for experimentally realizing and utilizing these notoriously elusive phenomena. Indeed, very recently, Vacuum-Rabi splitting of quantum dots embedded in a high- $Q$ cavity of a 2D slab-waveguide photonic crystal has been observed [195], thus opening a promising route to scalable solid-state-based quantum information processing. On the other hand-and more generally-engineering the density of states in strictly periodic or locally modified photonic crystals facilitates the realization of a "colored" photonic reservoir where individual emitters may coherently interact with a discrete number or even a continuum of modes. For instance, the latter regime is realized near photonic band edges and other van-Hove singularities that result from strong Bragg scattering. These multiple scattering effects lead to memory (or non-Markovian) effects and the emitters may experience strongly modified individual or collective dynamics.

Below, we will illustrate these categories with two of the most studied aspects of quantum optics in photonic crystals.

\subsection{Compact low-threshold photonic-crystal lasers}

Due to their high efficiency, large bandwidth, and compact size, semiconductor lasers have become indispensible for optical communication and sensing and many other applications. Realizing lasers in photonic-crystals provides significant potential for compact devices with low-lying laser thresholds that may be fully integrated into optoelectronic circuits. More precisely, the modes of high- $Q$ cavities in 2D and 3D photonic-crystals provide the smallest modal volumes in optical systems known to date and, therefore, ultra-compact lasers [188] have been among the first functional elements realized in photonic-crystals and recently continuous-wave operation at room temperature [371], lasing with very few (2-4) quantum dots as a gain medium [372], and ultra-fast modulation speeds far exceeding $100 \mathrm{GHz}$ [373] have been reported. Clearly, sensing as well as telecommunication application will benefit from these developments in the near future. Similarly, photonic-crystals have been employed as advanced mirrors for the end facets of traditional semiconductor laser resonators with notable improvement in the laser performance [374,375].

The ability of tailoring the properties of photonic-crystal cavities together with advances in the fabrication processes has recently lead to investigations of more complex photonic-crystal setups. For instance, a stable single-mode laser diode consisting of 40 coupled cavities in a hexagonal 2D photonic-crystal slab in InGaAsP/InP has been realized with a side-mode suppression greater than $40 \mathrm{~dB}$ and a maximal emission power of $2.6 \mathrm{~mW}$ at a laser wavelength of $1.54 \mu \mathrm{m}$ [376]. Similarly, coupled-cavity ring-like photonic-crystal lasers exhibiting stable single-mode lasing with side-mode suppression greater than $20 \mathrm{~dB}$ have been demonstrated [377]. Moreover, the recent demonstration of a optically triggered liquid-crystal infiltrated $Q$-switched photonic-crystal laser that may be reversibly switched between two orthogonally polarized modes [378] highlights the benefits of tailoring photonic-crystal cavities for synergetic integration of semiconductor nanophotonics with optical materials (see also Sections 2.1.1.1 and 2.1.3). Moreover, by combining photonic and electronic band structure engineering, surface-emitting quantum cascade microcavity lasers have been realized [379], thus providing a very interesting route to miniaturization and tailorable sources that may be employed for novel sensing and spectroscopy applications.

Finally, we want to note that photonic-crystal-based distributed feedback (DFB) lasers may be realized [380,381]. Using organic semiconductors as active material, these systems lend themselves to large-scale fabrication [380,382]. As a result of the distributed feedback from $2 \mathrm{D}$ photonic-crystals a lowering of the laser threshold relative to corresponding 
1D DFB lasers [380] and unusual emission characteristics [382] are observed, which may lead to applications in biosensing and lighting technology.

\subsection{Suppression and control of spontaneous emission in photonic-crystals}

An active material with a free space radiative transition that lies deep inside a PBG will be unable to emit a photon when placed inside a PBG material; instead, a photon-atom bound state is formed [383-385]. For frequencies near strong variations of the DOS such as photonic band edges or other van-Hove singularities that signal strong Bragg scattering, the emission dynamics will be modified relative to free space, due to the nontrivial structure of the atom's electromagnetic environment [386,385]. The resulting non-Markovian atom-field interaction has been predicted to give rise to a number of novel quantum optical phenomena, such as laser-like collective atomic emission [387] and atomic states that can be readily generated and protected from processes that would serve to decohere the system [388]. These are but a few of the novel phenomena associated with the suppression and control of spontaneous emission that can be achieved through PBG materials.

In a rotating wave approximation, the full quantum Hamiltonian for a two-level atom located at a position $\vec{r}_{0}$ of the Wigner-Seitz cell of an ideal photonic-crystal that is coupled to the electromagnetic can be written as [385]

$$
H=\frac{\hbar}{2} \omega_{21} \sigma_{z}+\hbar \sum_{\alpha} \omega_{\alpha} a_{\alpha}^{\dagger} a_{\alpha}+\mathrm{i} \hbar \sum_{\alpha}\left(g_{\alpha} a_{\alpha}^{\dagger} \sigma_{-}-g_{\alpha}^{*} \sigma_{+} a_{\alpha}\right) .
$$

The composite index $\alpha$ labels the band and wave vector of a given field mode (Bloch function), $\alpha \equiv\{n, \mathbf{k}\}$, and $a_{\alpha}^{\dagger}$ and $a_{\alpha}$ are the corresponding creation and annihilation operators for these modes, respectively. Furthermore, $\sigma_{j}$ $(j=+,-, z)$ are the usual Pauli operators for a two-level atom with a (bare) atomic resonance frequency $\omega_{21}$. The position-dependent atom-field mode coupling constants, $g_{\alpha}$, are [385]

$$
g_{\alpha}\left(\vec{d}, \vec{r}_{0}\right) \equiv g_{\alpha}=\omega_{21} \sqrt{\frac{1}{2 \hbar \epsilon_{0} \omega_{\alpha} V_{\mathrm{WSC}}}} \vec{d}_{2 \mathrm{LS}} \cdot \vec{E}_{\alpha}^{*}\left(\vec{r}_{0}\right),
$$

where $\vec{d}_{2 \text { LS }}$ represents the atom's dipole matrix element.

We wish to analyze the atomic emission in a Schrödinger equation formalism [386,385] that allows a particular transparent interpretation of the effects resulting from strong Bragg scattering in photonic-crystals. Atom-field interactions that involve more than one photon are more easily (and often necessarily) described by a density matrix or through Heisenberg operator equations-of-motion, and much of the following analysis can be carried over to such systems. In the single-photon sector of the atom-field Hilbert space, the wave function for a two-level atom is

$$
|\Psi\rangle=b_{2}\left(\vec{d}_{2 \mathrm{LS}}, \vec{r}_{0}, t\right)|2,\{0\}\rangle+\sum_{\alpha} b_{1, \alpha}\left(\vec{d}_{2 \mathrm{LS}}, \vec{r}_{0}, t\right)|1,\{\alpha\}\rangle \mathrm{e}^{-\mathrm{i} \Delta_{\alpha} t} .
$$

$b_{2}\left(\vec{d}_{2 \mathrm{LS}}, \vec{r}_{0}, t\right)$ and $b_{1, \alpha}\left(\vec{d}_{2 \mathrm{LS}}, \vec{r}_{0}, t\right)$, respectively, label the probability amplitudes for the excited atom plus an electromagnetic vacuum state, and a de-excited atom with a single photon in mode $\alpha$. In addition, we have introduced the abbreviation $\Delta_{\alpha}=\omega_{\alpha}-\omega_{21}$. In a frame that is co-rotating with the bare atomic resonance frequency, $\omega_{21}$, Eq. (4.87) along with the Hamiltonian (4.85) give the equations of motion for the amplitudes,

$$
\begin{aligned}
& \frac{\mathrm{d}}{\mathrm{d} t} b_{2}\left(\vec{d}, \vec{r}_{0}, t\right)=-\sum_{\alpha} g_{\alpha} b_{1, \alpha}\left(\vec{d}_{2 \mathrm{LS}}, \vec{r}_{0}, t\right) \mathrm{e}^{-\mathrm{i} \Delta_{\alpha} t}, \\
& \frac{\mathrm{d}}{\mathrm{d} t} b_{1}\left(\vec{d}_{2 \mathrm{LS}}, \vec{r}_{0}, t\right)=g_{\alpha} b_{2}\left(\vec{d}_{2 \mathrm{LS}}, \vec{r}_{0}, t\right) \mathrm{e}^{\mathrm{i} \Delta_{\alpha \cdot t}} .
\end{aligned}
$$

Formally integrating (4.89), substituting the solution into (4.88), and averaging over the dipole orientation, we arrive at an equation for the dipole-averaged excited state amplitude $b_{2}\left(\vec{r}_{0}, t\right)[385]$

$$
\frac{\mathrm{d}}{\mathrm{d} t} b_{2}\left(\vec{r}_{0}, t\right)=-\int_{0}^{t} \mathrm{~d} t^{\prime} G\left(\vec{r}_{0}, t-t^{\prime}\right) b_{2}\left(\vec{r}_{0}, t^{\prime}\right) .
$$


The time-delay Green function or memory kernel, $G\left(\vec{r}_{0, t}-t^{\prime}\right)$, describes the effect of the modified electromagnetic vacuum on the atomic system

$$
G(\vec{r}, \tau)=\Theta(\tau) \beta \int_{0}^{\infty} \mathrm{d} \omega \frac{N(\vec{r}, \omega)}{\omega} \mathrm{e}^{-\mathrm{i}\left(\omega-\omega_{21}\right) \tau} .
$$

Here, $N(\vec{r}, \omega)$ denotes the LDOS of the photonic-crystal at $\vec{r}$ and we have absorbed all numerical coefficients into the prefactor $\beta=\omega_{21}^{2} d_{2 \mathrm{LS}}^{2} / 12 \hbar \epsilon_{0} \pi^{2}$.

We would like to emphasize that-apart from the rotating-wave approximation-(4.90) is essentially exact and provides the basis for the fractional localization of the atomic population for atomic transition frequencies near a photonic band edge $[384,385]$ as well as for the anomalous Lamb shift of atomic transition frequencies which can easily lead to corrections of the normal Lamb shift that are several tens of percent in magnitude with both, positive and negative signs [385]. Owing to the strong variation in the LDOS of photonic-crystals, any approximation to (4.90) has to be carefully justified: For instance, given the rapidly oscillating exponent in the memory kernel (4.91), one is tempted to treat the LDOS near the bare atomic transition frequency $\omega_{21}$ as constant, take it outside the frequency integral and evaluate this integral to be proportional to a delta function in time. As a consequence, (4.90) would take on the form of a simple differential equation which can easily be solved by a decaying exponential function whose decay constant, i.e., rate of spontaneous emission, is proportional to the LDOS at frequency $\omega_{21}$. While this Wigner-Weiskopf-type (or Born-Markov-type) approximation is deceptively simple, it cannot be justified for frequencies near photonic band edges and other rapid variations (van-Hove singularities) in the LDOS (see Fig. 5 for a typical frequency dependence of the DOS in a high-index 3D photonic-crystal). In fact, the investigation of the dynamics and relaxation of quantum systems coupled to structured reservoirs has a rather long history and an illuminating discussion has been given in Ref. [389].

To date, a convincing experimental realization of the theoretically predicted non-Markovian dynamics discussed above is still absent. This may be attributed to the fact that until very recently, large-scale photonic-crystals of sufficiently high quality have not been available. In addition, non-Markovian effects may be masked rather easily by other (Markovian) relaxation processes. For instance, if the time scales associated with the non-radiative decay and dephasing processes of a given emitter species embedded in a photonic-crystal are shorter than the typical time scale of the corresponding memory kernel (4.91), it will be rather challenging to resolve the anticipated non-Markovian dynamics. The nonuniform angular emission characteristics of radiative sources located inside photonic-crystals further complicates the issue. Therefore, a careful characterization of both, the nonradiative properties of active material as well as the emission properties of radiative sources embedded in photonic-crystals, is a necessary prerequisite for a successful demonstration of non-Markovian effects in these systems and recent works [390-392] have reported progress in this direction.

\section{Metallo-dielectric periodic structures}

We have seen that large dielectric contrasts are highly desirable for photonic-crystals in order to achieve sizable complete photonic band gaps in particular and large resulting effects in general. In the optical regime, silicon with a permittivity of $\epsilon=12.9$ is already at the upper end of the scale for dielectrics. In contrast to this, metals can have negative permittivities ${ }^{6} \epsilon(\omega)$ with $|\epsilon(\omega)| \gg 1$ at frequencies $\omega$ well below the plasma frequency $\omega_{\text {pl }}$. With damping $\gamma$, the Drude model of free metal electrons with mass $m_{\mathrm{e}}$, charge $-e$, and density $N_{\mathrm{e}} / V$ yields

$$
\epsilon(\omega)=1-\frac{\omega_{\mathrm{pl}}^{2}}{\omega^{2}+\mathrm{i} \gamma \omega},
$$

with the plasma frequency

$$
\omega_{\mathrm{pl}}=\sqrt{\frac{e^{2} N_{\mathrm{e}}}{\epsilon_{0} V m_{\mathrm{e}}}} .
$$

\footnotetext{
${ }^{6}$ We have seen in (2.10) and (2.11) that $1 / \epsilon$ enters into the wave equations. Thus, negative values of $\epsilon$ can be considered as the continuation of $\epsilon=+\infty$.
} 
Table 1

Plasma frequency $\omega_{\mathrm{pl}}$ and damping frequency $\gamma$ of selected relevant metals

\begin{tabular}{lllr}
\hline & $\mathrm{Au}$ & $\mathrm{Ag}$ & $\mathrm{Al}$ \\
\hline$\omega_{\mathrm{pl}}$ & $2 \pi \times 2.08 \times 10^{15} \mathrm{~s}^{-1}$ & $2 \pi \times 2.18 \times 10^{15} \mathrm{~s}^{-1}$ & $2 \pi \times 3.11 \times 10^{15} \mathrm{~s}^{-1}$ \\
$\gamma$ & $2 \pi \times 1.96 \times 10^{13} \mathrm{~s}^{-1}$ & $2 \pi \times 5.08 \times 10^{12} \mathrm{~s}^{-1}$ & $2 \pi \times 4.35 \times 10^{13} \mathrm{~s}^{-1}$ \\
\hline
\end{tabular}

Values taken from Refs. [393,394].

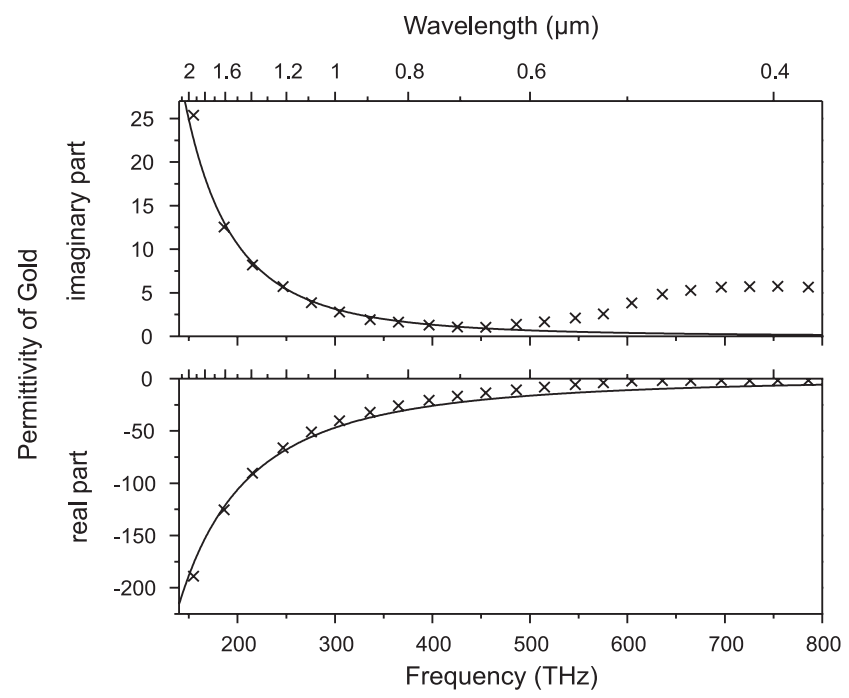

Fig. 37. Measured (crosses) real and imaginary parts of the permittivity $\epsilon(\omega)$ of gold. These data according to Ref. [393] are compared with the Drude model (solid curves) for the parameters given in Table 1. Note the good agreement for frequencies up to the interband transition onset frequency. Reprinted figure with permission of C. Enkrich.

Examples for typical parameters are given in Table 1. Often, for frequencies in the infrared or the near infrared, the Drude model is an excellent approximation of real metals. At higher frequencies, interband transitions [393] come into play in addition to the intraband transitions described by the Drude model. This can lead to significant modifications of $\epsilon(\omega)$. This is illustrated in Fig. 37 for the example of gold. From the metals quoted in Table 1, silver comes closest to a Drude metal within the visible part of the spectrum and has the lowest damping there. In contrast, Aluminum exhibits strong damping due to an interband transition at around $800 \mathrm{~nm}$ wavelength.

A more detailed description of metals also has to account for spatial dispersion [395], i.e., the permittivity $\epsilon(\omega, k)$ is not only a function of the frequency $\omega$ but also a function of the wave number of light $k$. We will briefly come back to this aspect in the context of Landau damping in Section 5.4.2.

For metals, it is often helpful to express the response of the electron system to an external field in terms of the current density $\vec{j}=\sigma(\omega) \vec{E}$ with the optical conductivity $\sigma(\omega)$ rather than by the optical polarization $\vec{P}$, hence $\epsilon(\omega)$. Within the Drude model, the connection is given by $\operatorname{Re}(\sigma(\omega))=\omega \epsilon_{0} \operatorname{Im}(\epsilon(\omega))$.

At first sight, one might be tempted to argue that metals are rather dull for photonics because a real $\epsilon<0$ (and $\mu=1$ ) implies a purely imaginary refractive index $n=+\sqrt{\epsilon}=\mathrm{i} \sqrt{|\epsilon|}$, hence opaque structures. We will see, however, that this is not correct at all for many periodic structures because the spatial distribution of the light field can adjust to avoid the metal, thus leading to highly transmittive structures-for certain wavelengths.

\subsection{Modified Planck's law in three-dimensional metallic photonic-crystals}

By proposing the concept of the photon, thereby introducing quantum optics, Einstein was able to derive Planck's law for the emission of a hot (bulk) body and eliminate the so-called ultraviolet catastrophe of classical electrodynamics. 
Assuming that the medium and the light field are in thermal equilibrium at the same temperature $T$, the general form for the energy density $u(\omega) \mathrm{d} \omega$ inside a medium and in a frequency interval $\mathrm{d} \omega$ is given by

$$
u(\omega) \mathrm{d} \omega \propto \frac{\omega}{\exp \left(\hbar \omega / k_{\mathrm{B}} T\right)-1} N(\omega) \mathrm{d} \omega .
$$

The factor $\omega$ in the numerator stems from the photon energy itself, the denominator originates from the thermal occupation factor of a mode at frequency $\omega$, i.e., the Bose factor, and the density of states (DOS) $N(\omega)$ (see Section 2.1.1.2) determines how many modes of the light field one finds in the frequency interval $\mathrm{d} \omega$ around frequency $\omega$. Only the latter aspect depends on the material under investigation. In a nondispersive bulk photonic medium, the density of states is given by

$$
N(\omega) \propto \omega^{2},
$$

which immediately leads to the usual form of Planck's law. For a dielectric PBG material (also see Section 2.1.1.2), the DOS would be zero within the photonic gap, hence, no emission is expected from this interval. As the total number of modes is conserved, this means that the DOS must have become larger in some other spectral region. For three-dimensional dielectric photonic crystals, these effects are generally not too large. In contrast, they can be quite dramatic for three-dimensional metallic photonic crystals. Generally, this is a rather difficult problem, hence, we start by discussing a very simple, yet instructive special case.

We consider a three-dimensional cubic lattice with lattice constant $a=l$ of air cubes of length $l$, the surface of which is coated with a thin film of ideal metal with conductivity $\sigma=\infty$. Thus, the electric field is zero everywhere on the metal and the interaction between adjacent cubes is strictly zero. Hence, we can first consider the field of a single isolated cube and add up the cubes at the end. The solution of the wave equation with these boundary conditions is simply given by standing waves in the cube and we have the discrete eigenfrequencies

$$
\omega=c_{0}|\vec{k}|=\omega_{N_{x} N_{y} N_{z}}=c_{0} \sqrt{\left(N_{x} \frac{\pi}{l}\right)^{2}+\left(N_{y} \frac{\pi}{l}\right)^{2}+\left(N_{z} \frac{\pi}{l}\right)^{2}}
$$

with the integer quantum numbers $N_{x}, N_{y}$, and $N_{z}$. Thus, the density of states is a series of $\delta$-peaks at the eigenfrequencies of the system, i.e.,

$$
N(\omega) \propto \sum_{N_{x} N_{y} N_{z}=1}^{\infty} \delta\left(\omega-\omega_{N_{x} N_{y} N_{z}}\right) .
$$

Obviously, the lowest eigenfrequency is given by

$$
\omega_{111}=c_{0} \sqrt{3} \frac{\pi}{l}
$$

the next higher one is threefold degenerate

$$
\omega_{211}=\omega_{121}=\omega_{112}=c_{0} \sqrt{6} \frac{\pi}{l}=\sqrt{2} \omega_{111} \text {, etc. }
$$

For example, if one is interested in blue-green emission at the fundamental mode, i.e., if $\hbar \omega_{111}=2.5 \mathrm{eV}$, the lattice constant has to be $a=l=430 \mathrm{~nm}$. The next higher mode would lie at photon energy $\hbar \omega_{211}=3.5 \mathrm{eV}$ (outside the visible). Thus, its Bose factor is lower than that of the fundamental mode by a factor of approximately $\exp \left(-1 \mathrm{eV} /\left(k_{\mathrm{B}} T\right)\right)$. For a temperature of $T=3000 \mathrm{~K}\left(k_{\mathrm{B}} T=0.26 \mathrm{eV}\right)$ - a typical surface temperature of an incandescent lamp-this factor is identical to about $3 \%$ (for each of the three degenerate frequencies $\omega_{211}=\omega_{121}=\omega_{112}$ ). For lower temperatures it would be yet smaller, higher temperatures are hardly compatible with the melting point of about any metal. We conclude that the thermal emission of this idealized metallic photonic-crystal would practically be monochromatic for all realistic temperatures. This is clearly different from the usual form of Planck's law in bulk media, leading to a broad continuous emission. The corresponding spectra are illustrated in Fig. 38.

For a real metal, the individual cubes would no longer be independent and the sharp peaks in the DOS are broadened by the formation of bands in analogy to the tight-binding approximation of solid-state physics. Indeed, most metals are far from being ideal at optical frequencies because typical plasma frequencies are not much above visible photon 


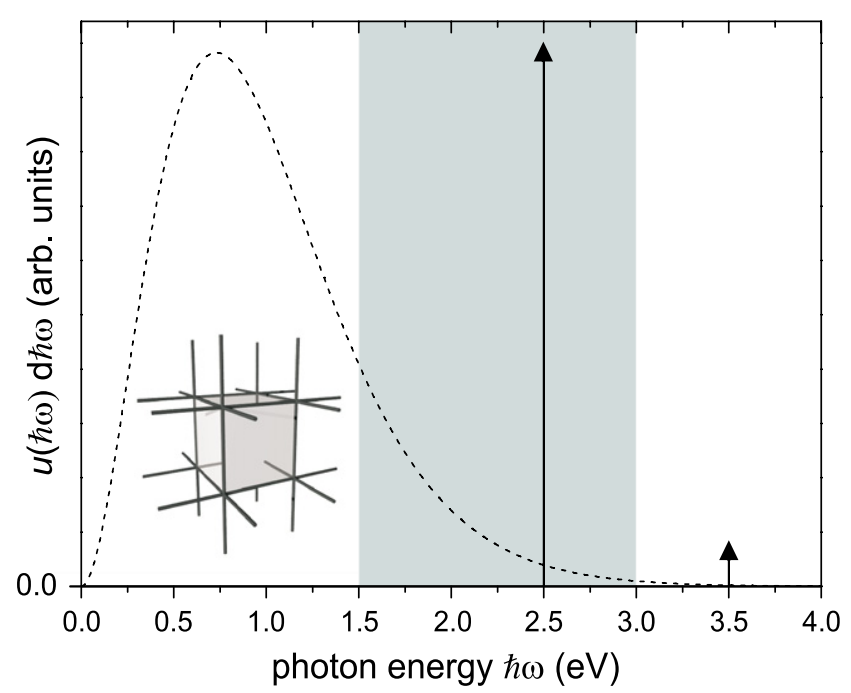

Fig. 38. Spectrally resolved electromagnetic energy density $u(\hbar \omega) \mathrm{d} \hbar \omega$ inside a three-dimensional simple cubic lattice of air cubes, each coated with a thin film of an ideal metal (see inset). Parameters are $a=430 \mathrm{~nm}$ and $T=3000 \mathrm{~K}$ (solid curve). The visible regime from $\hbar \omega=1.5$ to $3 \mathrm{eV}$ is highlighted by the gray area. This spectrum has to be compared to the corresponding energy density of black-body cavity radiation, i.e., the usual form of Planck's law (dashed). Be aware that this plot of the internal energy density must not be confused with the corresponding emission from the materials.

energies (see Table 1). Things become worse by the fact that the effective plasma frequency of the metallic photonic crystal is lower than in the bulk. In the long-wavelength limit, the light essentially "sees" an electron density which is reduced by the volume filling factor $f$ (with $0 \leqslant f \leqslant 1$ ) as compared to the bulk, i.e.,

$$
\frac{N_{\mathrm{e}}}{V} \rightarrow f \frac{N_{\mathrm{e}}}{V}
$$

Hence, the effective plasma frequency in (5.93) is lower than that of the bulk by the square root of the filling factor

$$
\omega_{\mathrm{pl}} \rightarrow \sqrt{f} \omega_{\mathrm{pl}} .
$$

One might be tempted to assume that this long-wavelength limit applies to metallic structures of arbitrary topology. However, from the microwave regime, exceptions are known. For example [396], a dense disordered collection of disconnected metallic spheres rather exhibits a Mie [397] resonance behavior (see Section 5.3) and no metallic cutoff. Other metallic microwave crystal structures have, e.g., been discussed in Ref. [398].

Having lighting applications in mind for metallic photonic crystals, it is interesting to investigate tungsten, the metal used in any (visible) incandescent lamp at temperatures around $T=3000 \mathrm{~K}$. However, despite all of its other virtues for conventional lighting, bulk tungsten is known to be far from an ideal black body and is often called a "gray-body" radiator, because its emissivity is about $50 \%$ in the visible.

Refs. [399-401] (see Fig. 39) discuss three-dimensional woodpile photonic crystals made from tungsten via a layer-by-layer direct-fabrication procedure analogous to that of the silicon woodpiles described in Section 2.2.3.1. The structures presented in Ref. [400] have a rod width of $0.5 \mu \mathrm{m}$, a rod spacing of $1.5 \mu \mathrm{m}$, and comprise 8 layers, equivalent to two lattice constants. The samples are heated by the Ohmic losses associated with an electrical current flowing through the tungsten. For an estimated [400] sample temperature of $T \approx 1500 \mathrm{~K}$, the measured thermal emission spectra reveal a sharp peak near $1.5-\mu \mathrm{m}$ wavelength and suppressed emission at longer wavelengths. The authors conclude that, compared to black-body radiation at the same temperature, the observed emission is narrower by a factor of nearly 2.5 .

One might be tempted to believe that this result implies that the emission from the metallic photonic crystal at the peak position is larger than that of an ideal (bulk) black body held at the same temperature [399,400]. A basic Gedanken experiment [402] along the lines of the derivation of Kirchhoff's law (emissivity equals absorbance) shows that this would allow for a periodically operating machine which just cools one heat bath. This would not be compatible with 


\section{a}

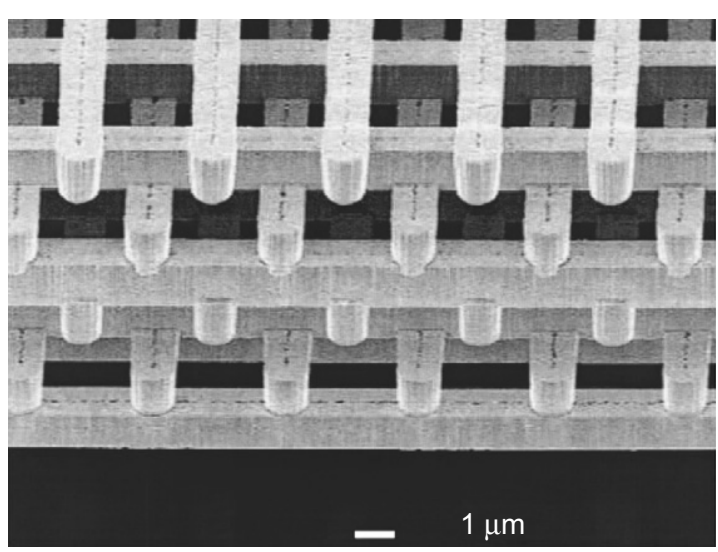

b

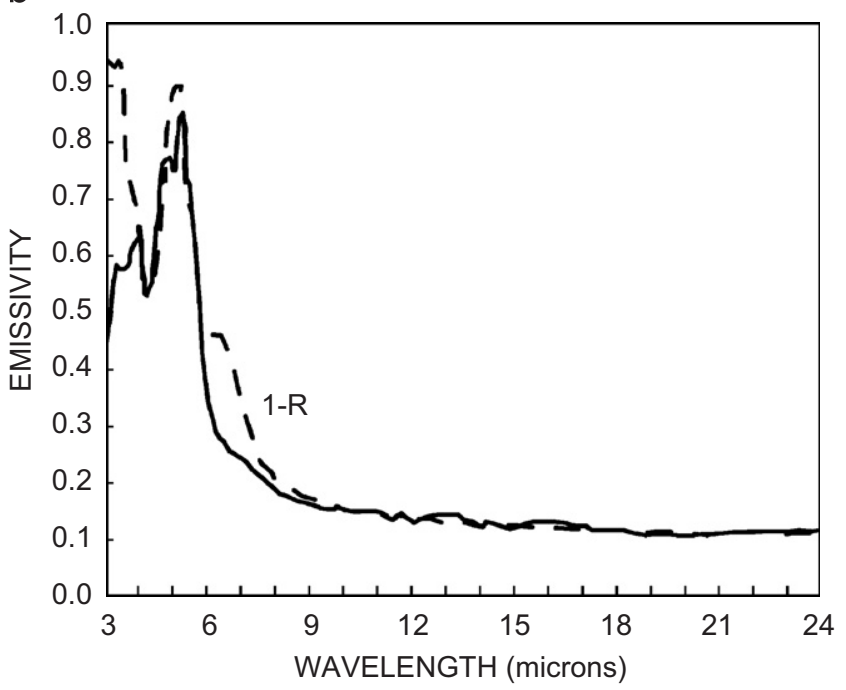

Fig. 39. (a) Electron micrograph of a three-dimensional tungsten woodpile photonic-crystal with a rod spacing of $4.2 \mu \mathrm{m}$. (b) Measured thermal emission from this structure at a temperature of $T=546 \mathrm{~K}$ (solid line). The dashed line gives measured values for $1-R$ at $T=300 \mathrm{~K}$. The emissivity and the specular reflectance were both measured at an angle of $17^{\circ}$ from normal incidence. Reprinted with permission from [401]. Copyright (2005), American Institute of Physics.

the second law of thermodynamics. Microscopically, the intense radiation inside the metallic photonic crystal would be strongly reflected when arriving at the interface to air because of the very large impedance mismatch. Thus, the emission in the peak would at best be as large as that of an ideal black body held at the same temperature [403] — provided that the metal electrons are in thermal equilibrium [404], as implied in Kirchhoff's law. (Accurate experimental determination of the metallic photonic-crystal temperature $T$ is a crucial aspect, but not trivial at all [401].) The energy density inside the medium at the spectral peak position, however, can be very large indeed. Specifically, it would be much larger than that for an ideal black body held at the same temperature (see Fig. 38). Also, the efficiency of a corresponding lamp, i.e., the ratio of emitted visible optical power and input electric power (for heating) can in principle approach $100 \%$ for a metallic photonic-crystal, which has to be compared with a mere $3 \%$ for a usual incandescent lamp at $T=3000 \mathrm{~K}$ temperature (derived from Planck's law).

In Ref. [405], opals (see Section 2.2.3.2) with silica sphere diameters of 850 and $550 \mathrm{~nm}$, respectively, have been coated with layers of tungsten with thicknesses ranging from zero to $50 \mathrm{~nm}$ using chemical vapor deposition (see Section 2.2.3.5). The original silica spheres were subsequently removed by wet chemical etching. For the correspondingly small lattice constants, the Bragg frequency could be pushed towards the near infrared and the visible. However, the 


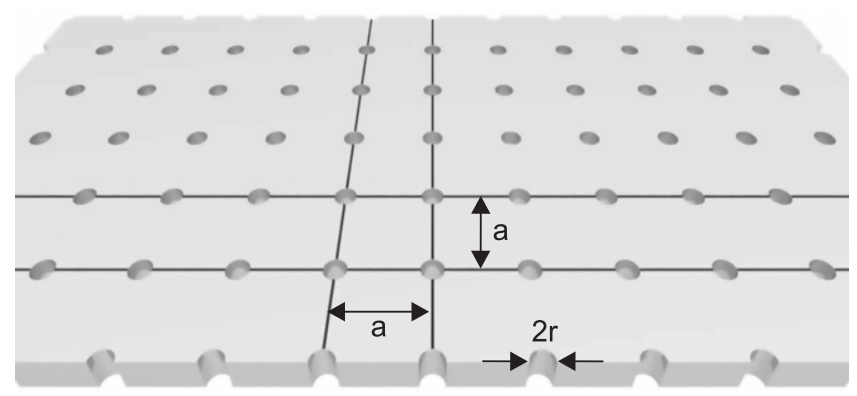

Fig. 40. Scheme of a metal film (gray) perforated with holes of radius $r$, arranged in a square lattice with lattice constant $a$ in the $x y$-plane.

measured reflectance and transmittance spectra revealed large effects of metal absorption, hindering the propagation of light throughout the photonic crystal, leading to a breakdown of the original band structure. Hence, the light rather samples single sphere cavities.

These findings, together with other more practical problems, such as the continuous evaporation and re-solidification of tungsten in some halogen incandescent lamps, pose severe challenges for using metallic photonic crystals in actual lighting applications. The modified thermal emission from metallic photonic crystals is, however, one of the few examples where pronounced quantum-optical effects from photonic crystals have been observed experimentally [399-401].

\subsection{Two-dimensional sub-wavelength hole arrays}

Consider a thin metal film perforated with holes of radius $r$, periodically arranged into a two-dimensional lattice with lattice constant $a$ (see Fig. 40) [406]. For simplicity, we consider a square lattice. In geometrical optics, the expected optical transmittance $T(\lambda)$ is simply given by the areal fraction of holes, i.e., by

$$
T(\lambda)=\frac{\pi r^{2}}{a^{2}},
$$

which is wavelength independent. This is, of course, too simplistic. The Bethe-Bouwkamp theory [407,408] of an isolated sub-wavelength size hole in an ideal metal film $(\sigma \rightarrow \infty)$ rather predicts that the transmittance scales with the sixth power of the ratio of hole radius and wavelength, i.e.,

$$
T(\lambda) \propto\left(\frac{r}{\lambda}\right)^{6}
$$

Hence, for small hole diameters, one naively expects a yet smaller transmittance than in geometrical optics.

Thus, it came as a surprise to many, when experiments on such structures [406] rather showed an optical transmittance larger than that expected from geometrical optics—-for particular resonance wavelengths. This result needed to be explained.

The key is a coupling to surface-plasmon-polaritons [409] via reciprocal lattice vectors of the periodic arrangement. The physics of surface polariton waves [61] will be discussed in a more general context in the section on magnetodielectrics below (see Section 5.4.1). Loosely speaking, these waves describe light crawling along the metal/air interface. Let us consider a metal/air interface (in the $x y$-plane), illustrated in Fig. 48(a), and consider a surface wave propagation along the $x$-direction. Under these conditions, the surface-plasmon-polariton dispersion relation is given by

$$
k_{x}^{\mathrm{sp}}(\omega)=\sqrt{\frac{\epsilon(\omega)}{1+\epsilon(\omega)}} \frac{\omega}{c_{0}}>\frac{\omega}{c_{0}} .
$$

The latter inequality holds if the dielectric function of the metal $(\gamma=0$ in $(5.92)), \epsilon(\omega)$, is smaller than -1 at the frequency of interest. Solving $\left|\vec{k}^{\mathrm{sp}}\right|=|\vec{k}|=\omega / c_{0}$ in the air half-space for the $z$-component of the 
surface-plasmon-polariton wave vector leads to

$$
\left(k_{z}^{\mathrm{sp}}\right)^{2}=\left(\frac{\omega}{c_{0}}\right)^{2}-\left(k_{x}^{\mathrm{sp}}\right)^{2}<0 .
$$

Thus, $k_{z}^{\mathrm{sp}}$ is purely imaginary, the wave is evanescent and is restricted to a narrow region around the interface. The inequality in (5.104) furthermore means that a plane wave with wave number $|\vec{k}|=\omega / c_{0}$ impinging onto the interface from the vacuum side cannot couple to the surface-plasmon polariton, because coupling requires both frequency (energy) and wave-vector (momentum) conservation. "The surface-plasmon-polariton wave number is too large." The positive side of this finding is that surface-plasmon polaritons allow for X-ray wavelengths $\lambda=2 \pi / k_{x}^{\mathrm{sp}}$ at optical frequencies $\omega$. This is the key idea underlying nanoplasmonics [410], aiming at delivering light to the nanoscale-which appears to be impossible at first sight in view of the large vacuum wavelength of light.

In the presence of a periodic arrangement of holes, however, the tangential component ( $x$-component) of the wave vector is only conserved modulo reciprocal lattice vectors $\pm 2 \pi / a$. For an incident wave vector of light in the $x y$-plane and with an angle of incidence $\alpha$ with respect to the surface normal this leads to the condition

$$
k_{x}^{\mathrm{sp}}=\frac{\omega}{c_{0}} \sin (\alpha)=\sqrt{\frac{\epsilon(\omega)}{1+\epsilon(\omega)}} \frac{\omega}{c_{0}} \pm N_{x} \frac{2 \pi}{a}
$$

with integer $N_{x}$. This condition can be met for combinations of special frequencies $\omega$ (or wavelengths) and angles of incidence $\alpha$. Loosely speaking, the incident light wave first couples to the surface-plasmon-polariton wave via reciprocal lattice vectors, subsequently moves along the interface and arrives at an aperture, "tunnels" through the aperture, couples to a surface-plasmon polariton on the other side of the perforated metal film, and is transmitted. This leads to enhanced (sometimes also called "extraordinary") transmittance for special parameter combinations (see Fig. 41). Indeed, the spectral positions observed in the experiments [406] are qualitatively reproduced by the simple equation (5.106). If the metal film is located on a substrate (e.g., glass), the surface-plasmon-polariton dispersion on the one side is obviously different from that on the other side. This asymmetry reduces the coupling, hence, the transmittance. Using symmetric structures, yet larger transmittance can be obtained in the spectral peaks [411]. Possibly, such structures can be used as spectral filters. More detailed theoretical work can be found in Refs. [412-415].

According to our above reasoning, the holes are not the only possibility for obtaining enhanced transmittance through the metal film for particular wavelengths and angles of incidence-any other periodic modulation associated with the metal film would do as well. Indeed, Refs. [416,417] discuss experiments on metal films without any holes, but with a periodic modulation of the substrate the metal film of constant thickness is located on. Again, the incident light can couple to the surface-plasmon-polariton wave via reciprocal lattice vectors (for certain wavelengths and angles), resulting in transmittance maxima. Such maxima also occur for corresponding dielectric periodic structures [418], for which large transmittance is no surprise.

However, Bragg reflection is only one part of the story on the perforated hole arrays as it turns out that the shape of the holes has a significant influence on the transmittance as well [419]. In this work, the authors explicitly show that the transmittance is significantly different for circular, rectangular, and square-shaped apertures. Intuitively, it is clear that a spectral resonance of the hole, possibly even coinciding with a Bragg resonance, can drastically influence the throughput. It is this general double-resonance aspect which we want to discuss in the following section. Resonances of metallic objects (be it holes or particles) without Bragg resonances will also play the major role in Section 5.4 on metamaterials.

As we now switch from holes in metal films to metallic particles-which are the "negative" (or complement) of the holes-it is interesting to briefly address the relation between the two. Babinet's principle [142,420] strictly connects the two for two-dimensional planar structures made from infinitesimally thin ideal metal films in air or vacuum. Here, current flow is obviously restricted to $z=0$ in the $x y$ plane. Let us denote the positive structure with "+" and the negative one with "-". (The "+" structure plus the "-" structure gives a continuous film.) The incident waves impinge onto the $x y$ plane from the LHS, i.e., from $z<0$. For the positive structure, the "incident" fields shall be given by $\vec{E}_{+}(x, y,-0), \vec{B}_{+}(x, y,-0)$, the "transmitted" fields by $\vec{E}_{+}(x, y,+0), \vec{B}_{+}(x, y,+0)$. The negative structure shall be illuminated by a wave for which the role of the incident electric field and magnetic field is interchanged with respect to the positive structure, i.e., $\vec{E}_{-}(x, y,-0)=c_{0} \vec{B}_{+}(x, y,-0)$ and $\vec{B}_{-}(x, y,-0)=-1 / c_{0} \vec{E}_{+}(x, y,-0)$. Under these 


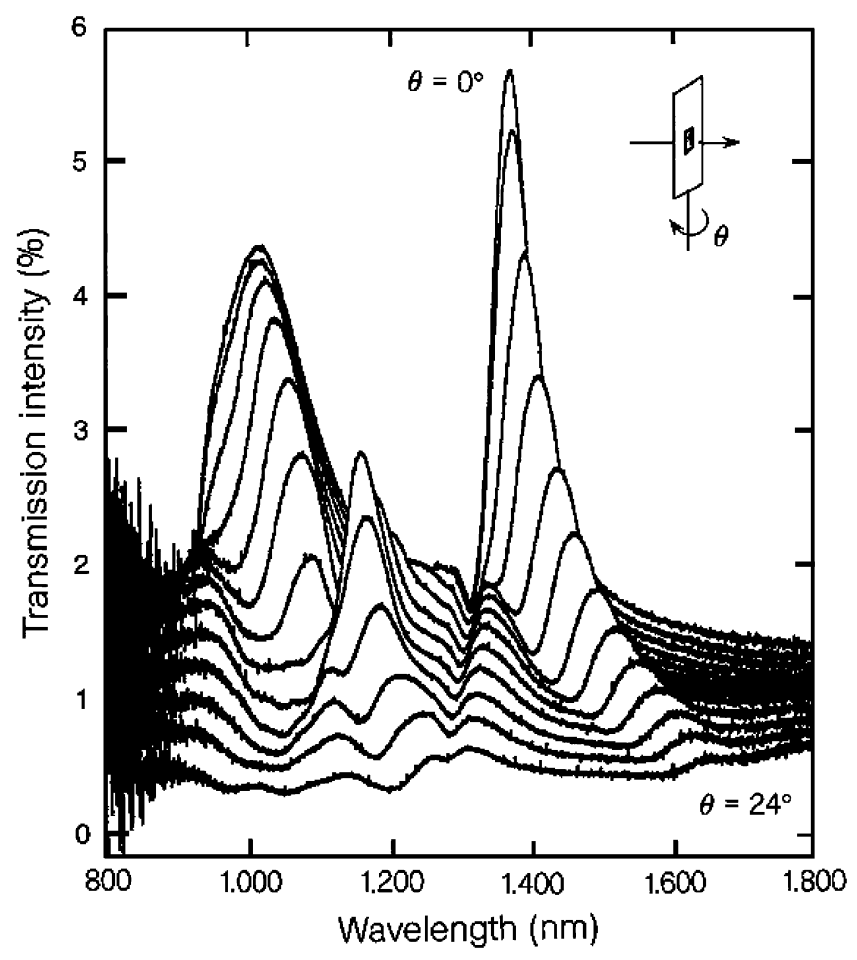

Fig. 41. Measured transmittance spectra of a sub-wavelength hole array for different angles of incidence $\alpha$ with respect to the surface normal. Sample parameters are: $a=900 \mathrm{~nm}$, hole diameter $2 r=150 \mathrm{~nm}$, and silver film thickness $t=200 \mathrm{~nm}$. Thus, according to geometrical optics, the transmittance should equal $T=\pi r^{2} / a^{2} \approx 2 \%$. Note the much larger transmittance in the peaks. Compare with (5.106). Reprinted figure with permission from [406]. Copyright (1998) by Nature Publishing Group.

conditions, Babinet's principle allows to compute the "transmitted" fields $\vec{E}_{-}(x, y,+0)$ and $\vec{B}_{-}(x, y,+0)$ for the negative structure via the relations

$$
\vec{E}_{+}(x, y,+0)-c_{0} \vec{B}_{-}(x, y,+0)=\vec{E}_{+}(x, y,-0)
$$

and

$$
c_{0} \vec{B}_{+}(x, y,+0)+\vec{E}_{-}(x, y,+0)=c_{0} \vec{B}_{+}(x, y,-0) .
$$

While Babinet's principle quantitatively only applies to structures made from infinitesimally thin ideal metal films, Ref. [420] showed by example that it qualitatively applies for real metal films of finite thickness in the microwave regime as well. Approaching optical frequencies, the behavior of actual metals deviates more and more from an ideal metal (see Table 1 and Fig. 37). Thus, Babinet's principle in the above form should be taken with caution in photonics.

\subsection{Metallic photonic-crystal slabs}

In Section 2, we have discussed photonic-crystals made by a periodic arrangement of a dielectric material with frequency-independent refractive index and air. We have seen that, for frequencies near the Bragg-frequency, a stop band or even a band gap can evolve. Furthermore, we have seen that additional resonance effects arise for a constituent material with a frequency-dependent refractive index $n(\omega)$, e.g., from a Lorentz-oscillator (or two-level system) resonance (also see Section 3.1). The physics of metallic photonic-crystal slabs [421-424] is related to this.

To see how a Lorentz-oscillator response arises for a small metallic object, we briefly remind ourselves on the particle plasmon (or Mie) resonance [397,425]. The dimension of the metal nanoparticle, $2 r$, shall be much smaller than the wavelength of light, $\lambda$, i.e., $2 r \ll \lambda$. This is equivalent to the limit $\lambda \rightarrow \infty$. With the vacuum dispersion relation of 
light, $\lambda f=c_{0}$, this is further equivalent to the limit of vanishing frequency $f$ of light, $f \rightarrow 0$. In other words, a small particle exposed to an electromagnetic plane wave is equivalent to a particle between the plates of a plate capacitor in electrostatics. Here, the external electric field of the plate capacitor, $\vec{E}_{\text {ext }}$, induces a polarization $\vec{P}$ in the particle, which gives rise to surface charges leading to the well-known depolarization field $\vec{E}_{\mathrm{dp}}$. In general, the depolarization field is a complicated function of the polarization, i.e.,

$$
\epsilon_{0} \vec{E}_{\mathrm{dp}}=-\mathbf{N}_{\mathrm{dp}} \vec{P}
$$

with the depolarization matrix $\mathbf{N}_{\mathrm{dp}}$. For the special case of a sphere, the depolarization matrix inside the sphere reduces to a number and we have $\mathbf{N}_{\mathrm{dp}} \rightarrow N_{\mathrm{dp}}=1 / 3$. This allows us to write

$$
\vec{P}=\epsilon_{0} \chi \vec{E}=\epsilon_{0} \chi\left(\vec{E}_{\mathrm{ext}}+\vec{E}_{\mathrm{dp}}\right)=\epsilon_{0} \chi \vec{E}_{\mathrm{ext}}-\frac{1}{3} \chi \vec{P} .
$$

Solving for the polarization with respect to the external electric field and introducing $\epsilon(\omega)=1+\chi(\omega)$ leads to

$$
\vec{P}(\omega)=3 \epsilon_{0} \frac{\epsilon(\omega)-1}{\epsilon(\omega)+2} \vec{E}_{\mathrm{ext}}(\omega)=\epsilon_{0} \chi_{\mathrm{eff}}(\omega) \vec{E}_{\mathrm{ext}}(\omega)
$$

This expression obviously has a resonance for $\epsilon(\omega)=-2$. (Note that the frequency dependence does not contradict at all the above electrostatic approximation, which is meaningful as long as the corresponding wavelength is large compared with the particle size.) Upon inserting the permittivity (5.92) of a Drude metal with plasma frequency $\omega_{\mathrm{pl}}$ and damping $\gamma$, we obtain for the effective permittivity $\epsilon_{\text {eff }}(\omega)=1+\chi_{\text {eff }}(\omega)$

$$
\epsilon_{\mathrm{eff}}(\omega)=1+\frac{\omega_{\mathrm{pl}}^{2}}{\omega_{\text {Mie }}^{2}-\omega^{2}-\mathrm{i} \gamma \omega},
$$

a Lorentz oscillator form with the Mie resonance frequency

$$
\omega_{\mathrm{Mie}}=\frac{1}{\sqrt{3}} \omega_{\mathrm{pl}} .
$$

For typical metals like gold or silver, the resonance frequency $\omega_{\text {Mie }}$ lies in or near the visible part of the spectrum. If the spherical particle is embedded in a dielectric medium with permittivity $\epsilon_{\text {med }}$, the particle plasmon resonance condition becomes $\epsilon(\omega)=-2 \epsilon_{\text {med }}$. For particles outside the electrostatic approximation, the overall qualitative behavior remains the same, but the resonance frequency is lowered with increasing particle size. This gradually evolves into an antennalike resonance for particle sizes approaching half the wavelength of light. Particle shapes other than spheres lead to modified resonance frequencies via their respective depolarization factors $N_{\mathrm{dp}}$. The corresponding straightforward generalization of (5.113) is given by $\omega_{\mathrm{Mie}}=\sqrt{N_{\mathrm{dp}}} \omega_{\mathrm{pl}}$.

We now consider a photonic-crystal made from such small metal structures. Fig. 42 schematically shows one possible structure, where metal nanowires are arranged into a one-dimensional lattice with lattice constant $a$. Importantly, an appreciable coupling between the plasmon resonance and the Bragg resonance requires an additional slab waveguide, e.g., underneath the metal nanoparticles. Therefore, the physics of metallic photonic-crystal slabs is distinct from that of usual metallic gratings, which have been discussed extensively many years ago [426]. Using nanowires rather than spheres or dots offers the interesting possibility to conveniently control the coupling to light via the polarization of the incident light: If the electric-field vector is oriented perpendicular to the nanowires (TM-polarization), a pronounced depolarization field arises, giving rise to a strong optical resonance at $\omega_{\text {Mie }} \neq 0$. In contrast, if the electric field vector is along the wire axis (TE-polarization), the depolarization factor is zero, i.e., $N_{\mathrm{dp}}=0 \Rightarrow \omega_{\mathrm{Mie}}=0$, and one rather gets a Drude-type response of the metal from (5.112).

In Fig. 43 we depict measured optical extinction spectra (the negative logarithm of the optical transmittance) for both TE and TM polarization. For TE polarization, the observed sharp extinction peak simply shifts with lattice constant. This peak is due to Bragg diffraction of the incident light into the waveguide mode, leading to reduced transmittance in the forward direction. The behavior for the TM polarization is much more complex. Here, two resonances, the particle plasmon and the Bragg resonance interact and lead to a pronounced avoided crossing. While the avoided crossing is expected for any two interacting harmonic oscillators (Lorentz oscillators), the actual lineshape of the extinction spectra reminds one of Fano lineshapes in quantum mechanics. These lineshapes have been reproduced by 

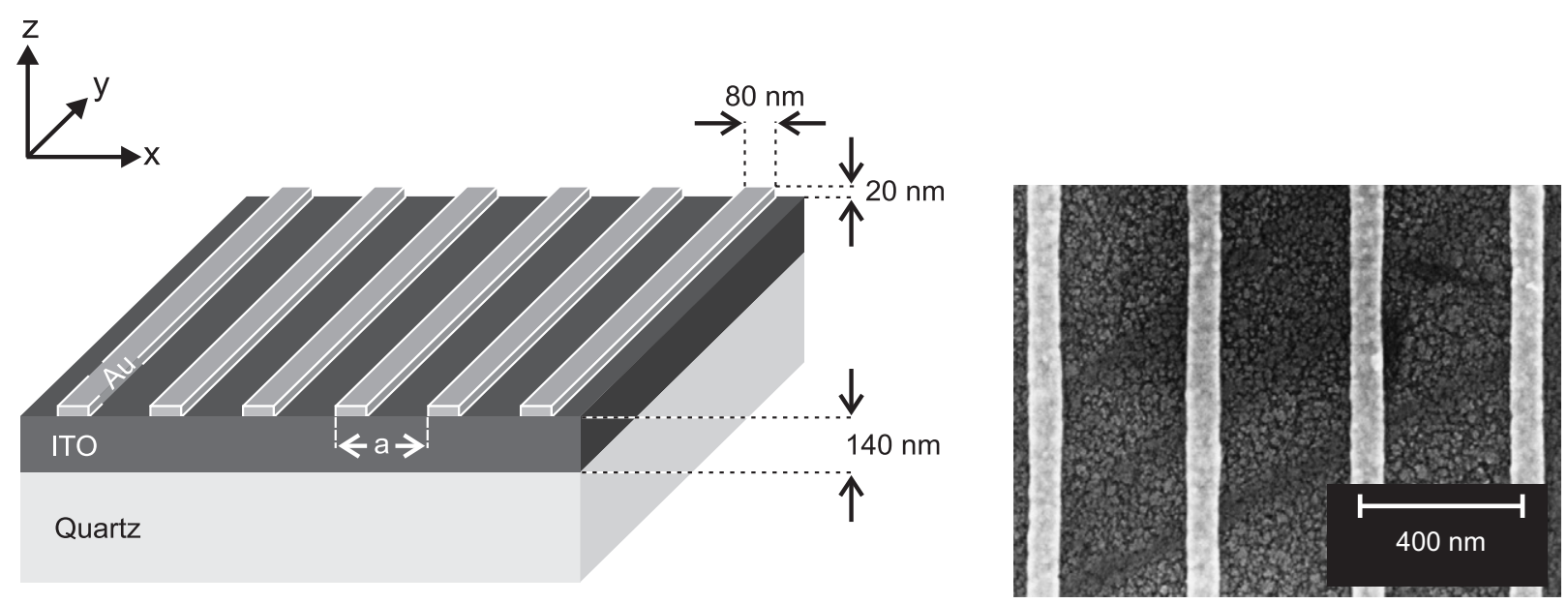

Fig. 42. LHS: Scheme of a one-dimensional metallic photonic-crystal slab, RHS: electron micrograph of an actual structure with lattice constant $a=400 \mathrm{~nm}$. After [423].

a

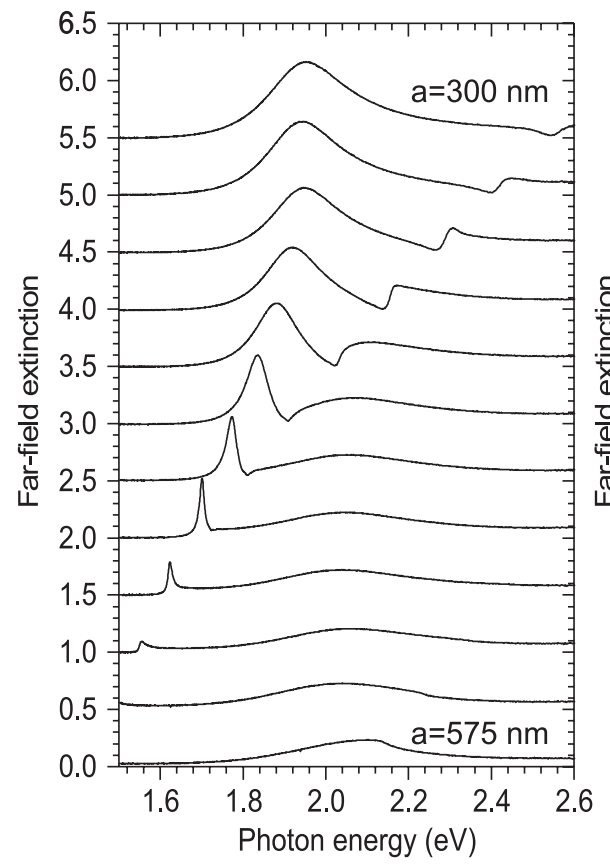

b

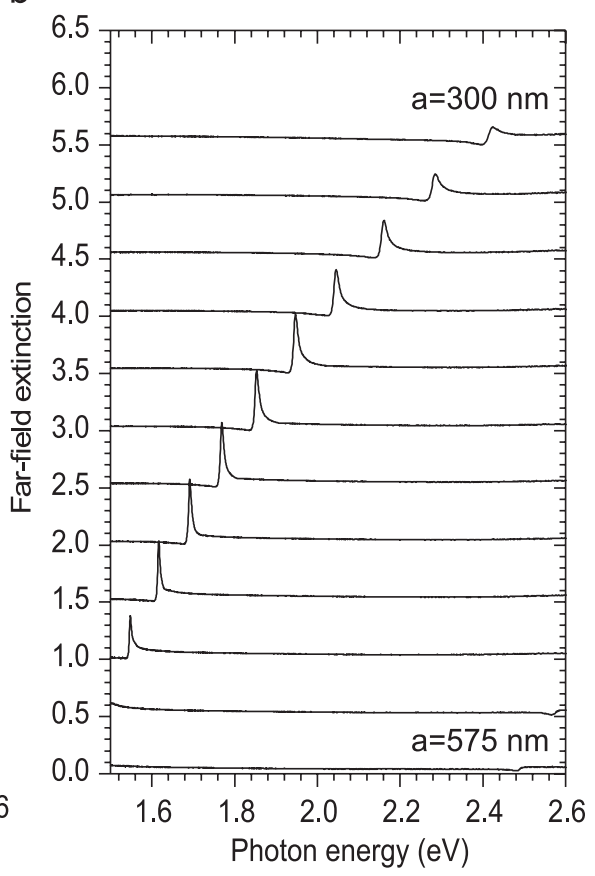

Fig. 43. Normal-incidence extinction spectra for two orthogonal linear polarizations and for different lattice constants $a$ from 300 to $575 \mathrm{~nm}$ in steps of $25 \mathrm{~nm}$. The curves for different values of $a$ are shifted for clarity. LHS: TE-polarization, i.e., incident electric field vector along the metal nanowires, RHS: TM-polarization, i.e., electric field vector perpendicular to the wires. After [423].

numerical calculations of the extinction spectra using a scattering-matrix approach [422,423] (see Section 2.1.4.2). In the following, we show that these features can also be explained on a much simpler and more general footing [424]. Broadly speaking, they are an interference phenomenon occurring for any two interacting oscillators, one of which is spectrally much narrower than the other one. 
a

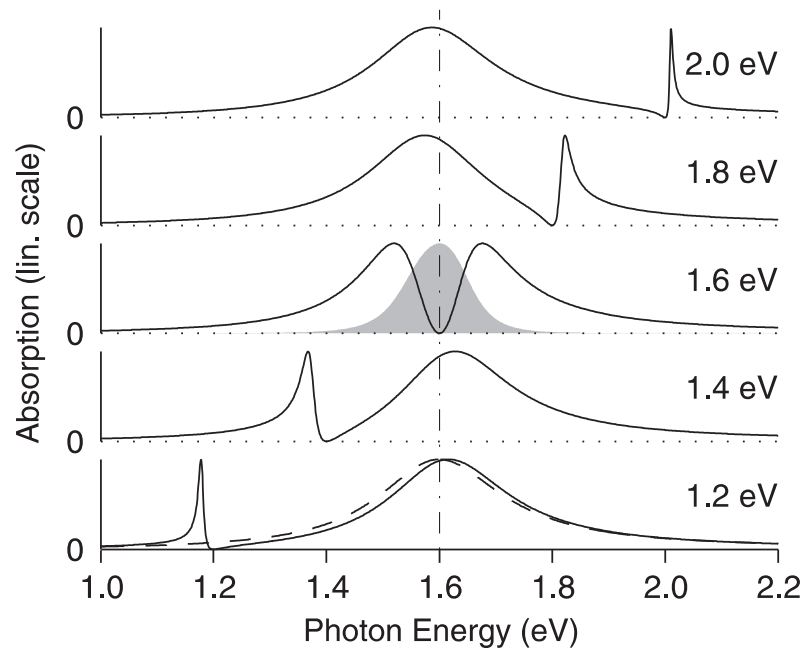

b

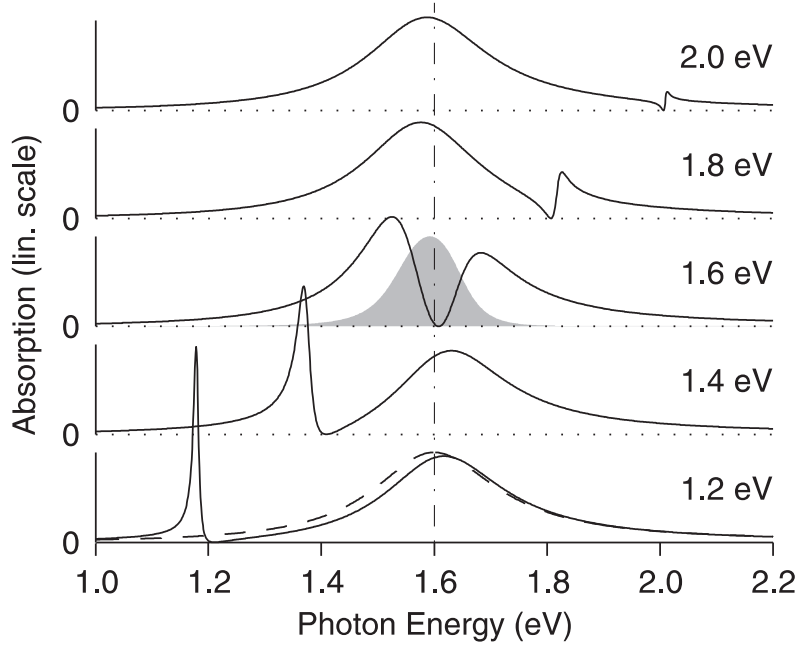

Fig. 44. Absorption coefficient $\alpha$ versus photon energy $\hbar \omega$ according to (5.116). (a) $q_{\mathrm{wg}} / q_{\mathrm{pl}}=0$, and (b) $q_{\mathrm{wg}} / q_{\mathrm{pl}}=+0.1$. Common parameters are $\hbar \Omega_{\mathrm{pl}}=1.6 \mathrm{eV}, \gamma_{\mathrm{pl}}=1 /(5 \mathrm{fs})$, and $\hbar \Omega_{\mathrm{c}}=0.5 \mathrm{eV}$. The "naked" waveguide photon energy $\hbar \Omega_{\mathrm{wg}}$ runs from $1.2 \mathrm{to} 2.0 \mathrm{eV}$ in steps of $0.2 \mathrm{eV}$. Note the avoided crossing and the Fano-like lineshapes. These spectra can be compared with the experiment shown on the RHS of Fig. 43. Taken from [424].

Consider two coupled oscillating particles of equal mass $m$. Newton's second law can easily be arranged into the form

$$
\begin{aligned}
& \ddot{x}_{\mathrm{pl}}+2 \gamma_{\mathrm{pl}} \dot{x}_{\mathrm{pl}}+\Omega_{\mathrm{pl}}^{2} x_{\mathrm{pl}}-\Omega_{\mathrm{c}}^{2} x_{\mathrm{wg}}=\frac{q_{\mathrm{pl}}}{m} E(t), \\
& \ddot{x}_{\mathrm{wg}}+2 \gamma_{\mathrm{wg}} \dot{x}_{\mathrm{wg}}+\Omega_{\mathrm{wg}}^{2} x_{\mathrm{wg}}-\Omega_{\mathrm{c}}^{2} x_{\mathrm{pl}}=\frac{q_{\mathrm{wg}}}{m} E(t) .
\end{aligned}
$$

Here, $x_{\mathrm{pl}}(t)$ and $x_{\mathrm{wg}}(t)$ are the displacements representing the particle plasmon and waveguide oscillations, respectively. The resonance frequencies, half-widths at half-maximum, and charges (oscillator strengths) of the uncoupled system are denoted by $\Omega_{j}, \gamma_{j}$, and $q_{j}\left(j=\mathrm{pl}\right.$, wg), respectively. $\Omega_{\mathrm{c}}^{2}$ represents the coupling strength between the oscillators. We consider the limit $\gamma_{\mathrm{wg}} \ll \gamma_{\mathrm{pl}}$ or $\gamma_{\mathrm{wg}}=0$, which is crucial for the occurrence of Fano-like resonances. Following along the usual lines of Lorentz oscillators, it is straightforward to derive the absorption coefficient

$$
\alpha(\omega)=\frac{N_{\mathrm{pl}} q_{\mathrm{pl}}^{2}}{V 2 m \epsilon_{0} c_{0}} \frac{4 \gamma_{\mathrm{pl}} \omega^{2}\left(\omega^{2}-\Omega_{\mathrm{wg}}^{2}-\left(q_{\mathrm{wg}} / q_{\mathrm{pl}}\right) \Omega_{\mathrm{c}}^{2}\right)^{2}}{\left(\left(\omega^{2}-\Omega_{\mathrm{pl}}^{2}\right)\left(\omega^{2}-\Omega_{\mathrm{wg}}^{2}\right)-\Omega_{\mathrm{c}}^{4}\right)^{2}+4 \gamma_{\mathrm{pl}}^{2} \omega^{2}\left(\omega^{2}-\Omega_{\mathrm{wg}}^{2}\right)^{2}},
$$

which mimics the experimental extinction spectra. $N_{\mathrm{pl}}$ is the number of plasmon/waveguide oscillator pairs in volume $V$. Examples of absorption spectra are shown in Fig. 44. One obtains the anticipated avoided crossing. For $q_{\mathrm{wg}} \ll q_{\mathrm{pl}}$, absorption maxima appear at the spectral positions $\omega$ given by

$$
\omega^{2}=\frac{\Omega_{\mathrm{pl}}^{2}+\Omega_{\mathrm{wg}}^{2}}{2} \pm \sqrt{\frac{\left(\Omega_{\mathrm{pl}}^{2}-\Omega_{\mathrm{wg}}^{2}\right)^{2}}{4}+\Omega_{\mathrm{c}}^{4}}
$$

These positions coincide with the normal mode frequencies of the coupled, but undamped system. In contrast to frequent believe, however, the lineshape does not correspond to the sum of two effective Lorentz oscillators. One rather gets a highly asymmetric, Fano-like lineshape. Usually, a Fano resonance results from the coherent interaction of a discrete quantum mechanical state with a continuum of states. In the purely classical model discussed here, a single spectrally sharp oscillator coherently interacts with a strongly homogeneously broadened second oscillator. The latter replaces the continuum (i.e., a broad inhomogeneous distribution). One result of the Fano-like interaction is that one obtains zero absorption in between the two absorption maxima. The position of this zero appears at the root of the numerator of (5.116), e.g., for $q_{\mathrm{wg}} \approx 0$, at the spectral position of the (uncoupled) waveguide mode, $\Omega_{\mathrm{wg}}$. Intuitively, this minimum 
is a result of destructive interference, which effectively suppresses the response of the two absorption "channels", the polarizations of which have a phase difference near $\pi$. In other words, the electric field has nodes on the metallic nanowires (or dots).

For nonlinear or near-field optical experiments on such metallic photonic-crystal slab waveguide structures we refer the reader to Refs. [424,427] and Ref. [423], respectively. The case of replacing the electric-dipole Mie resonance discussed here by a magnetic-dipole resonance has been realized in Ref. [428].

In the limit $\Omega_{\mathrm{wg}} \gg \Omega_{\mathrm{pl}}$, equivalent to small lattice constants $a$ in the RHS column of Fig. 43, one obtains an effective medium exhibiting a Lorentz oscillator resonance. Such metamaterials will be discussed in more detail next.

\subsection{Metamaterials}

We have seen that the lattice constant of a photonic-crystal is on the order of the wavelength of light, whereas the lattice constants of atomic crystals are orders of magnitude smaller than the wavelength. As a result, the light field effectively averages over these fine atomic structures and "sees" an effective homogeneous medium (also see corresponding discussion in Section 2.1.1.1).

Metamaterials lie in between these two limits. They are artificial periodic structures with lattice constants that are still smaller than the wavelength of light. Similarly, the light field again "sees" an effective homogeneous material. The "atoms", however, are functional building blocks which are composed of many real atoms (mostly metallic). This allows for tailoring their electromagnetic properties by design in ways that would not be possible with real atoms, hence, for example, allowing to realize electric as well as magnetic dipoles at optical frequencies.

To appreciate the impact of magnetic resonances at optical frequencies, it is instructive to recall that many optics textbooks set the magnetic permeability $\mu$ to unity within the first ten pages (as in this review in Section 2). The authors usually reason this by explaining that materials with $\mu \neq 1$ in the optical range do not occur in nature. Subsequently, hundreds of pages are devoted to the rich variety of optical phenomena for this special case. Presenting ways to realize materials with $\mu \neq 1$, especially with $\mu<0$, opens up a wealth of new possibilities.

\subsubsection{Electrodynamics of magneto-dielectrics}

To further motivate the enriched possibilities with such metamaterials, we start by discussing a few basic but actually not-so-well-known aspects of electromagnetism for the case of $\epsilon(\omega) \neq 1$ and $\mu(\omega) \neq 1$. We do not yet ask how such materials can be made-but we will see in Section 5.4.2 that they can be made indeed by periodic nanostructures (or, alternatively, by nonperiodic "glassy" structures as well). Much of the following goes back to the pioneering work by Veselago [49,429], who in 1968 addressed the "what-if" question: What would happen if both the electric permittivity and the magnetic permeability were negative at some frequency?

Example 1. First, we recall that the impedance of such an isotropic material is given by

$$
Z(\omega)=\sqrt{\frac{\mu_{0} \mu(\omega)}{\epsilon_{0} \epsilon(\omega)}}=Z_{0} \sqrt{\frac{\mu(\omega)}{\epsilon(\omega)}} .
$$

When light impinges from vacuum or air onto the material under normal incidence, a part of the wave will be reflected if and only if the material impedance $Z(\omega)$ is different from the vacuum impedance ${ }^{7} Z_{0}$. Only for the special case $\mu(\omega)=1$ this is equivalent to saying that reflection occurs if and only if the material has an index of $n(\omega) \neq 1$. Generally, zero reflectance can also occur for $n(\omega) \neq 1$, e.g., for $\epsilon(\omega)=\mu(\omega)=2$. Furthermore, even for $n(\omega)=1$, nonzero reflection can occur, e.g., for $\epsilon(\omega)=2$ and $\mu(\omega)=1 / 2$.

Often, the metamaterials to be described below are not available in the form of half-space geometries but are rather fabricated as a thin film between, e.g., vacuum or air and a dielectric substrate. One can describe this geometry as a film of thickness $d$ sandwiched between an incident half-space (with impedance $Z_{\mathrm{i}}$ and refractive index $n_{\mathrm{i}}>0$ ) and transmitted half-space (with impedance $Z_{\mathrm{t}}$ and refractive index $n_{\mathrm{t}}>0$ ). Using the usual transfer-matrix approach of

\footnotetext{
${ }^{7}$ In fact, this has also been the basis for the perfectly matched layers discussed in Section 2.1.4.3.
} 
wave optics and considering normal incidence, it is straightforward to obtain the complex field reflectivity $r(\omega)$ and the complex field transmittivity $t(\omega)$ given by

$$
t(\omega)=\frac{2}{\left(1+z_{\mathrm{r}}\right) \cos \varphi-\mathrm{i}\left(z_{\mathrm{r}} z+z^{-1}\right) \sin \varphi}
$$

and

$$
r(\omega)=\frac{t(\omega)}{2}\left(\left(1-z_{\mathrm{r}}\right) \cos \varphi-\mathrm{i}\left(z_{\mathrm{r}} z-z^{-1}\right) \sin \varphi\right)
$$

Here, we have introduced the phase $\varphi$ which the wave acquires due to propagation over the length $d$,

$$
\varphi(\omega)=\frac{\omega}{c_{0}} n(\omega) d,
$$

and the abbreviations $z_{\mathrm{r}}=Z_{\mathrm{i}} / Z_{\mathrm{t}}$ and $z=Z(\omega) / Z_{\mathrm{i}}$. For a free-standing film or a film embedded in one material, where the material "i" equals the material " $\mathrm{t}$ ", thus $z_{\mathrm{r}}=1$, and $n_{\mathrm{t}}=n_{\mathrm{i}}$, these expressions simplify to those given in Refs. $[430,431]$.

Note, that one can compute the impedance $Z(\omega)$ and the refractive index $n(\omega)$ of the magnetodielectric material of interest from the knowledge of both $r(\omega)$ and $t(\omega)$. Mathematically, however, different solutions are possible because of the different branches of the trigonometrical functions. Thus, additional physical boundaries must be imposed. From $Z(\omega)$ and $n(\omega)$ one can then compute $\epsilon(\omega)$ and $\mu(\omega)$. This procedure allows to "retrieve" effective complex electric permittivities and complex magnetic permeabilities, from numerically calculated reflectance and transmittance spectra of complicated structures. Such a parameter retrieval is important and common for the magnetic and negativeindex metamaterials to be discussed below. The same procedure is also possible with measured spectroscopic data. Be aware, however, that knowledge of the phases of $t(\omega)$ and $r(\omega)$ is crucial, i.e., knowledge of the intensity reflectance $R(\omega)=|r(\omega)|^{2}$ and the intensity transmittance $T(\omega)=n_{\mathrm{t}} / n_{\mathrm{i}}|t(\omega)|^{2}$ alone is not sufficient. We will come back to this aspect in Section 5.4.2.

Example 2. Next, we consider reflection of a plane wave under oblique incidence from vacuum or air onto a material half-space. For dielectrics with negligible absorption and for $\mu(\omega)=1$, the Fresnel formulae tell us that the reflectance is zero for p-polarization, i.e., for an electric-field vector in the plane of incidence, if the angle of incidence $\alpha$, measured with respect to the surface normal, is given by the Brewster angle $\alpha_{\mathrm{B}}^{\mathrm{p}}$ with

$$
\tan \left(\alpha_{\mathrm{B}}^{\mathrm{p}}\right)=n(\omega)=\sqrt{\epsilon(\omega)}
$$

The intuitive origin is that electric dipoles do not emit along their oscillation axis, which, for the Brewster angle, coincides with the wave vector of the reflected wave. For the orthogonal s-polarization at the same angle, the reflectivity is finite.

What happens for real $\mu(\omega) \neq 1$ ? For p-polarization, the generalization [432] of (5.122) is

$$
\tan \left(\alpha_{\mathrm{B}}^{\mathrm{p}}\right)=\sqrt{\frac{\epsilon^{2}(\omega)-\epsilon(\omega) \mu(\omega)}{\epsilon(\omega) \mu(\omega)-1}} .
$$

For s-polarization, where the Brewster angle "usually" does not occur at all under the above conditions, one obtains [432]

$$
\tan \left(\alpha_{\mathrm{B}}^{\mathrm{S}}\right)=\sqrt{\frac{\mu^{2}(\omega)-\epsilon(\omega) \mu(\omega)}{\epsilon(\omega) \mu(\omega)-1}} .
$$

For the special case of $\epsilon(\omega)=1$, this simplifies to

$$
\tan \left(\alpha_{\mathrm{B}}^{\mathrm{s}}\right)=n(\omega)=\sqrt{\mu(\omega)},
$$

i.e., we expect a "magnetic" Brewster angle for s-polarization which is closely similar to the usual "electric" Brewster angle for p-polarization. The intuitive origin is that magnetic dipoles do not emit along their oscillation axis either. For 
the Brewster angle, this axis coincides with the wave vector of the reflected wave. As the role of electric and magnetic components has reversed, the role of s- and p-polarization also reverses. In the more general case of $\epsilon(\omega) \neq 1$ and $\mu(\omega) \neq 1$, one has to use the above general formulae which deliver two generally different Brewster angles $\alpha_{\mathrm{B}}^{\mathrm{p}}$ and $\alpha_{\mathrm{B}}^{\mathrm{s}}$.

Example 3. Consider refraction of light at the interface between vacuum (or air) and a medium with permittivity $\epsilon(\omega)=-1$ and magnetic permeability $\mu(\omega)=-1$ at frequency $\omega>0$. The impedance of this medium is obviously $Z=Z_{0}$. Thus, as pointed out in Example 1, a light wave impinging from vacuum onto this medium would be completely transmitted, no reflected wave occurs for any angle of incidence. This aspect makes the detailed discussion of the different components of the electromagnetic vectors very transparent: As usual, we obtain from the first and the third Maxwell equations, respectively, that the components of $\vec{D}$ and $\vec{B}$ normal to the surface are continuous (i.e., they do not exhibit discontinuities). From the second and the fourth Maxwell equation we obtain that the tangential components of $\vec{E}$ and $\vec{H}$ are also continuous. Let us first consider p-polarization of light, i.e., the electric-field vector lies in the plane of incidence. In this case, we have for the tangential (t) and normal (n) components of $\vec{E}$ and $\vec{B}$, respectively: $E_{\mathrm{t}} \rightarrow E_{\mathrm{t}}, E_{\mathrm{n}} \rightarrow-E_{\mathrm{n}}, B_{\mathrm{t}} \rightarrow-B_{\mathrm{t}}$, and $B_{\mathrm{n}}=0$. This situation is visualized in Fig. 45. As always (induction law), $\vec{k}$, $\vec{E}$, and $\vec{B}$ form a right-handed system but $\vec{S}, \vec{E}$, and $\vec{B}$ form a "left-handed" system on the RHS of Fig. 45. Hence, materials of this sort are sometimes called left handed. We avoid this phrase in this review because "handedness" has a well-defined meaning in the context of chirality, which can lead to confusion. In other words, the wave vector of light $\vec{k}$ and the Poynting vector $\vec{S}$ point in opposite directions (see Section 2). In this sense, the phase velocity of the wave inside the medium is negative (we will see below that the corresponding group velocity can be positive or negative). Also, the light wave is refracted towards the "wrong" side of the surface normal, which is consistent when assuming a negative index of refraction in Snell's law. Hence, in the special case considered, we have to take the minus sign in $n(\omega)= \pm \sqrt{\epsilon(\omega) \mu(\omega)}$ if both $\epsilon(\omega)$ and $\mu(\omega)$ are real and negative, i.e.,

$$
n=-\sqrt{(-1)(-1)}=-1
$$

In what follows, we want to call materials with $n<0$ negative-index materials. The discussion for s-polarization, where the electric-field vector is normal to the plane of incidence, is analogous: $E_{\mathrm{t}} \rightarrow E_{\mathrm{t}}, E_{\mathrm{n}}=0, B_{\mathrm{t}} \rightarrow-B_{\mathrm{t}}$, and $B_{\mathrm{n}} \rightarrow B_{\mathrm{n}}$. This leads to the same conclusions as for the p-polarization. Movies and photorealistic images of objects in negative-index materials, obtained via ray tracing, are presented in Ref. [433].

We note in passing that the momentum density vector $\vec{D} \times \vec{B}$, i.e., the electromagnetic momentum per volume, is parallel to the local Poynting vector $\vec{S}=\vec{E} \times \vec{H}$ both inside vacuum and inside the negative-index material under the above conditions. Thus, photon momentum $\vec{p}$ and wave vector of light $\vec{k}$ are no longer parallel in negative-index materials, i.e., $\vec{p}=\hbar \vec{k}$ is not valid, but rather $\vec{p}=-\hbar \vec{k}$ holds.

Strictly speaking, the Kramers-Kronig relations [142]—which reflect causality—demand that any dispersive response in $\epsilon(\omega)$ and/or $\mu(\omega)$ is accompanied by an imaginary part. Indeed, negative real parts of the permittivity and permeability are a sufficient, but not a necessary condition for a negative real part of the refractive index: For complex electric permittivity $\epsilon(\omega)=\epsilon^{\prime}(\omega)+\mathrm{i} \epsilon^{\prime \prime}(\omega)$ and complex magnetic permeability $\mu(\omega)=\mu^{\prime}(\omega)+\mathrm{i} \mu^{\prime \prime}(\omega)$, the complex

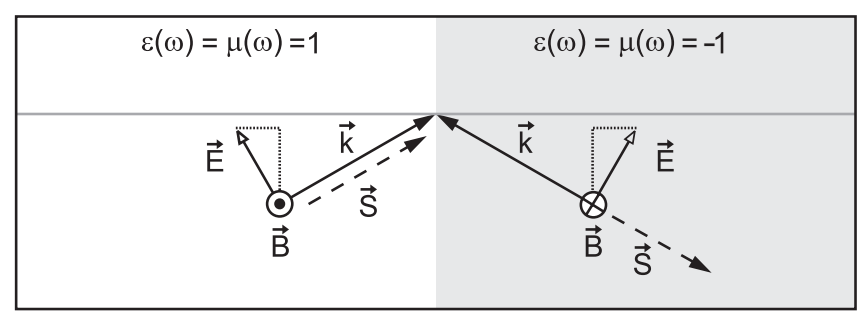

Fig. 45. Illustration of negative refraction at the interface between a vacuum or air half-space on the LHS and a negative-index material with $\epsilon(\omega)=\mu(\omega)=-1$ on the RHS for p-polarization of the incident light. The resulting negative refraction leads to a refractive index of $n(\omega)=-\sqrt{\epsilon(\omega) \mu(\omega)}=-1$. Also note that the wave vector (solid) and the Poynting vector (dashed) of light inside the negative-index material are opposite to one another. In this sense, the phase velocity of light is negative indeed. 
refractive index $n(\omega)=n^{\prime}(\omega)+\mathrm{i} n^{\prime \prime}(\omega)$ is given by [434]

$$
n(\omega)=\sqrt{|\epsilon(\omega) \| \mu(\omega)|} \exp \left(\frac{\mathrm{i}}{2}\left[\operatorname{arccot}\left(\frac{\epsilon^{\prime}}{\epsilon^{\prime \prime}}\right)+\operatorname{arccot}\left(\frac{\mu^{\prime}}{\mu^{\prime \prime}}\right)\right]\right) .
$$

This equation can be derived by expressing the complex numbers $z=z^{\prime}+z^{\prime \prime}$ as $z=|z| \exp (\mathrm{i} \varphi)$. By further using the form $\varphi=\operatorname{arccot}\left(z^{\prime} / z^{\prime \prime}\right)$ (rather than $\varphi=\arctan \left(z^{\prime \prime} / z^{\prime}\right)$ ), the condition $n^{\prime \prime}(\omega) \geqslant 0$, which is required from energy conservation in a passive medium for all frequencies $\omega$, is automatically fulfilled. Constraints for $\epsilon^{\prime \prime}(\omega)$ and $\mu^{\prime \prime}(\omega)$ will be discussed below. We will see that they can be negative. Regions of negative $n^{\prime}(\omega)$ can alternatively be identified by inspection of the imaginary part of the equation $n^{2}=\epsilon \mu$, i.e.,

$$
\left(n^{\prime}(\omega)+\mathrm{i} n^{\prime \prime}(\omega)\right)^{2}=\left(\epsilon^{\prime}(\omega)+\mathrm{i} \epsilon^{\prime \prime}(\omega)\right)\left(\mu^{\prime}(\omega)+\mathrm{i} \mu^{\prime \prime}(\omega)\right),
$$

which immediately leads to

$$
n^{\prime}(\omega)=\frac{\epsilon^{\prime}(\omega) \mu^{\prime \prime}(\omega)+\epsilon^{\prime \prime}(\omega) \mu^{\prime}(\omega)}{2 n^{\prime \prime}(\omega)} .
$$

From $n^{\prime \prime}(\omega) \geqslant 0$ we see that $n^{\prime}(\omega)$ becomes negative if and only if the numerator is negative, i.e.,

$$
\operatorname{Re}(n(\omega))<0 \Leftrightarrow\left(\epsilon^{\prime}(\omega) \mu^{\prime \prime}(\omega)+\epsilon^{\prime \prime}(\omega) \mu^{\prime}(\omega)\right)<0 .
$$

Fig. 46 illustrates these aspects by assuming a damped Lorentz oscillator resonance for the electric permittivity

$$
\epsilon(\omega)=1+\frac{\omega_{\mathrm{pl}, \mathrm{e}}^{2}}{\Omega_{\mathrm{e}}^{2}-\omega^{2}-\mathrm{i} \gamma_{\mathrm{e}} \omega}
$$

and a roughly similar form to be discussed below (see (5.161)) for the magnetic permeability

$$
\mu(\omega)=1+\frac{f \omega^{2}}{\Omega_{\mathrm{m}}^{2}-\omega^{2}-\mathrm{i} \gamma_{\mathrm{m}} \omega} .
$$

The real parts of $\epsilon(\omega), \mu(\omega)$, and $n(\omega)$ are shown in Fig. 46 for the case of finite damping. Fig. 47 shows the corresponding dispersion relation of light, $\omega=k c_{0} / n^{\prime}(\omega)$, for the case of negligible damping, i.e., $\gamma_{\mathrm{e}}=\gamma_{\mathrm{m}}=0$. While the wave vector, and hence the phase velocity of light, is negative in the frequency region where $n^{\prime}(\omega)<0$, the slope of the dispersion curve, and thus the group velocity, is positive whenever wave propagation is allowed (caution: this changes for finite damping, see discussion below). In the gray areas, the real part of the wave vector is zero, hence wave propagation is not allowed, and the transmittance of a bulk material is zero.

Note that the negative refraction in a photonic-crystal metamaterial with negative permittivity and negative permeability is distinct from the negative refraction in a photonic crystal as discussed in Section 2.1.2.1. The latter is actually due to diffraction, or in other words, it arises from interference of partial waves from different lattice points, whereas in a metamaterial the light effectively averages over the different "photonic atoms".

Example 4. In the previous example, we have learned about the unusual dispersion relation of waves propagating in the bulk of a magnetodielectric material. Here, we consider corresponding surface waves at the interface of such material and vacuum (or air). Such surface waves play a crucial role for the "perfect lens" to be discussed in Example 5.

In this example, for clarity, we again assume real $\epsilon(\omega)$ and $\mu(\omega)$. For purely dielectric materials as well as metals, i.e., for $\mu(\omega)=1$, it is well known that surface waves can result from the oscillatory electric dipoles distributed in the interface plane as shown in Fig. 48(a). Here, the electric-field vector lies in the plane spanned by the direction of propagation and the surface normal (p-polarization). (For materials with $\mu=1$, there are no solutions for which $\vec{E}$ has a component normal to this plane.) We make the ansatz for the magnetic field vector in air

$$
\vec{H}(\vec{r}, t)=\vec{H}_{0} \mathrm{e}^{\mathrm{i}\left(k_{x}^{\mathrm{sp}} x+k_{z}^{\mathrm{sp}} z-\omega t\right)}+\text { c.c. }
$$

Here, we have chosen the coordinate system such that $k_{y}^{\mathrm{sp}}=0 . \vec{H}_{0}$ is oriented along the $y$-direction, i.e., $\vec{H}_{0}=\left(0, H_{0}, 0\right)^{\mathrm{T}}$. Introducing this ansatz into the second Maxwell equation immediately delivers the corresponding electric field, which 


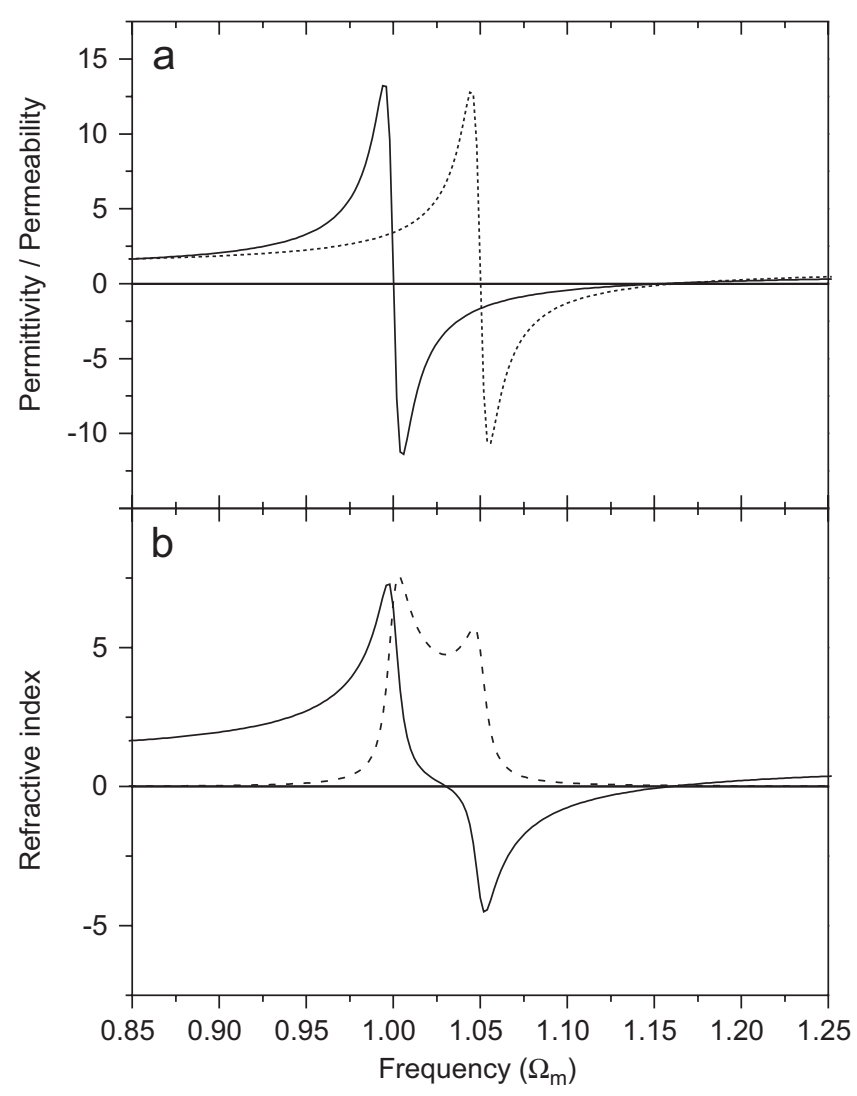

Fig. 46. Example for the real part of the permittivity $\epsilon(\omega)$ (dotted) and permeability $\mu(\omega)$ (solid). (b) Resulting refractive index $n(\omega)$ according to (5.131) and (5.132). The solid (dashed) curve is the real (imaginary) part. Parameters are: $\Omega_{\mathrm{e}} / \Omega_{\mathrm{m}}=1.05, \omega_{\mathrm{pl}, \mathrm{e}} / \Omega_{\mathrm{m}}=0.5, f=0.25, \gamma_{\mathrm{e}} / \Omega_{\mathrm{m}}=0.01$, and $\gamma_{\mathrm{m}} / \Omega_{\mathrm{m}}=0.01$.

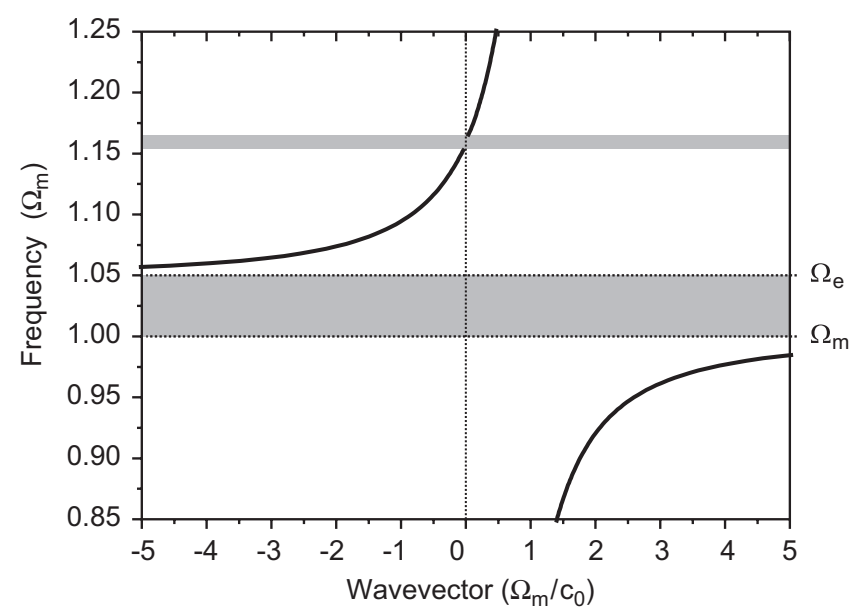

Fig. 47. Dispersion relation of light corresponding to the parameters shown in Fig. 46, except that the damping is set to zero. Only those parts of the dispersion relation are shown to which an incident wave impinging from the left (i.e., with positive wave vector) can couple to. 
a

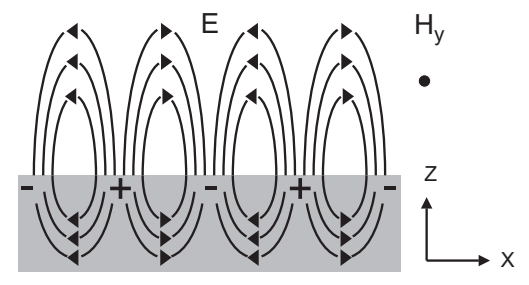

b

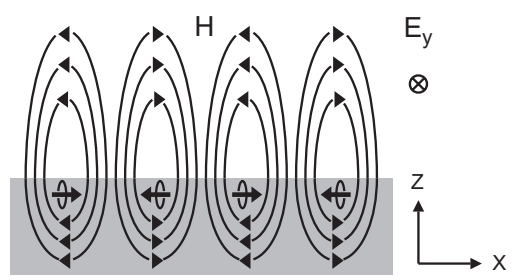

Fig. 48. Illustration of surface waves at the interface between a material and air (or vacuum). (a) $\mu(\omega)=1$ and p-polarization, (b) $\epsilon(\omega)=1$ and s-polarization.

has components along $x$ and $z$, but none along $y$ (see Fig. 48). Together with the continuity of the normal components of $\vec{D}$ and $\vec{B}$ and the tangential components of $\vec{E}$ and $\vec{H}$, this leads to the dispersion relation of (dielectric) surface polaritons (equal to (5.104)) [61]

$$
k_{x}^{\mathrm{sp}}=\sqrt{\frac{\epsilon(\omega)}{1+\epsilon(\omega)}} \frac{\omega}{c_{0}} .
$$

Following Ref. [435], the generalization of this well-known result for p-polarization and $\mu(\omega)=1$ to $\mu(\omega) \neq 1$ is

$$
k_{x}^{\mathrm{sp}}=\sqrt{\frac{\epsilon^{2}(\omega)-\mu(\omega) \epsilon(\omega)}{\epsilon^{2}(\omega)-1}} \frac{\omega}{c_{0}} .
$$

From the surface polariton dispersion relation together with $\left|\vec{k}^{\mathrm{sp}}\right|=\omega / c_{0}$ in air (or vacuum) we get $\left(k_{z}^{\mathrm{sp}}\right)^{2}=$ $\left(\omega / c_{0}\right)^{2}-\left(k_{x}^{\mathrm{sp}}\right)^{2}<0$, which means that the wave is exponentially decaying or increasing normal to the interface (z-direction). With nothing in close proximity to the interface, the boundary conditions exclude the exponentially increasing solution.

What about the s-polarization? Here, the electric-field component of the light is normal to the plane spanned by the propagation direction and the surface normal. For a purely magnetic material, i.e., for $\epsilon(\omega)=1$, these surface waves can be visualized by replacing the electric dipoles shown in Fig. 48(a) by the magnetic dipoles shown in Fig. 48(b). Due to the symmetry of electric and magnetic fields in the Maxwell equations, the corresponding dispersion relation for s-polarization is given by

$$
k_{x}^{\mathrm{sp}}=\sqrt{\frac{\mu(\omega)}{1+\mu(\omega)}} \frac{\omega}{c_{0}} .
$$

Following Ref. [435], the generalization of this result for s-polarization to $\epsilon(\omega) \neq 1$ is

$$
k_{x}^{\mathrm{sp}}=\sqrt{\frac{\mu^{2}(\omega)-\mu(\omega) \epsilon(\omega)}{\mu^{2}(\omega)-1}} \frac{\omega}{c_{0}} .
$$

The dispersion relations (5.135) and (5.137) are illustrated by example in Fig. 49. Here, we have introduced $\epsilon(\omega)$ and $\mu(\omega)$ according to (5.131) and (5.132) with the parameters given in the caption of Fig. 49.

Example 5. What are the consequences of negative refraction addressed in Example 3? We consider an infinite (parallel) plate of thickness $d$ made of the above medium with $\epsilon(\omega)=\mu(\omega)=n(\omega)=-1$, embedded in vacuum (or air) [436]. Fig. 50 shows selected rays from an object on the LHS of this plate at a distance of $a$ from the plate. This leads to a real image of the object on the RHS of the plate with a distance $a^{\prime}$ given by

$$
d=a+a^{\prime}
$$




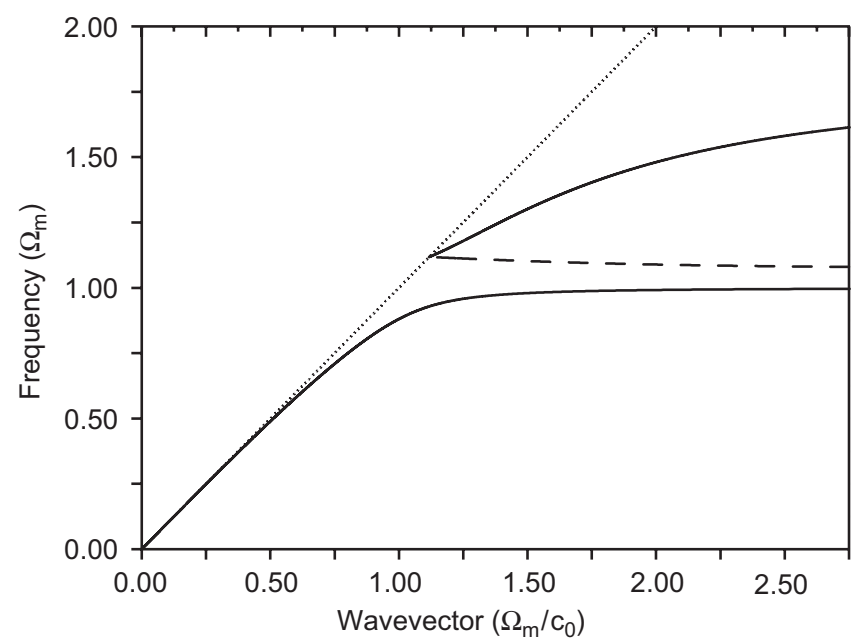

Fig. 49. Illustration of the surface-polariton-dispersion relation at the interface between a magneto-dielectric material and air (or vacuum). p-polarization (solid curves), s-polarization (dashed curve). Note that there are no s-polarized surface waves for a purely dielectric material, i.e., for $\mu(\omega)=1$. Parameters are: $\omega_{\mathrm{pl}, \mathrm{e}} / \Omega_{\mathrm{m}}=2.5, f=0.25, \Omega_{\mathrm{e}}=0$ (i.e., Drude model), and $\gamma_{\mathrm{e}}=\gamma_{\mathrm{m}}=0$.

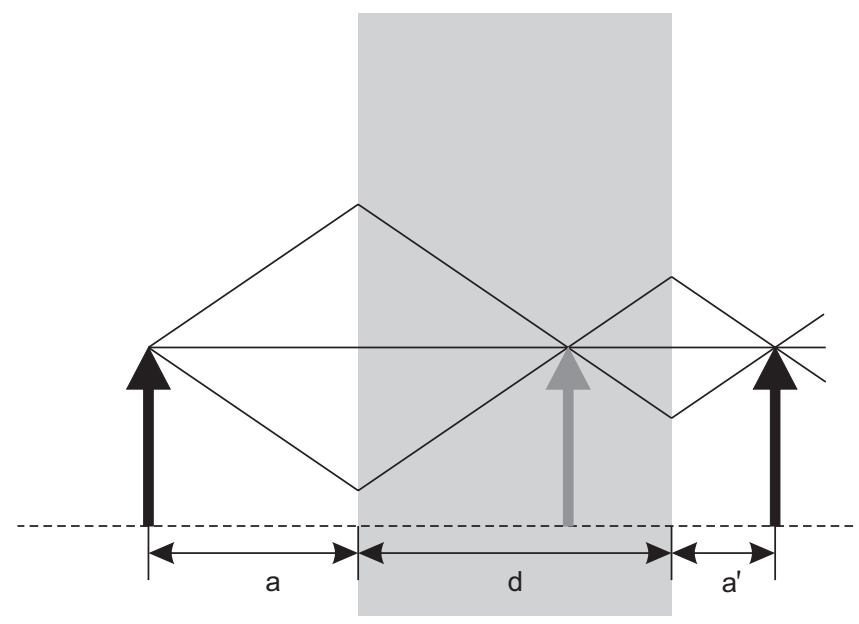

Fig. 50. Illustration of a "perfect lens". The gray plate with thickness $d$ in the center is a negative-index material with $\epsilon(\omega)=\mu(\omega)=n(\omega)=-1$ (see Fig. 45), the outer parts are vacuum or air with $\epsilon=\mu=n=+1$. Ref. [436] showed that the sharpness of the image (RHS black arrow) of the object (LHS black arrow) is not restricted by the usual diffraction limit.

as indicated in Fig. 50, provided that $d>a$. For $d \leqslant a$, the image is virtual, i.e., $a^{\prime}<0$. This behavior is "reversed" with respect to that for a usual "thin" spherical lens with focal length $f_{1}$ with

$$
\frac{1}{f_{1}}=\frac{1}{a}+\frac{1}{a^{\prime}}
$$

where $a$ is the distance between the object and the lens and $a^{\prime}$ the distance between the lens and the image of the object. For $a>f_{1}$, the image is real, for $a \leqslant f_{1}$, it is virtual, i.e., $a^{\prime}$ is negative. To obtain a real image outside the lens, the object must not be too close to the lens for a usual lens, whereas it must not be too far from the lens for a "perfect lens". Obviously, the parallel plate with $n=-1$ yields a 1:1 magnification. A wave analysis [436] for a twodimensional lens shows that such a "perfect lens" can beat the usual diffraction limit, i.e., the image can have significant sub-wavelength resolution. Why? Any sub-wavelength structure of the object on the LHS leads to partial waves which exponentially decay away from the object (evanescent waves). In a usual far-field microscope, these evanescent waves have practically decayed to zero in the image plane. Hence, the information on these fine sub-wavelength structure is 
lost. The interface between vacuum and the negative-index material leads to surface waves (see Example 4). As pointed out above, one obtains two solutions: One for which the amplitude exponentially decays away from the interface and one which exponentially increases. For an isolated interface, the decaying contribution alone fulfills the boundary conditions, thus, the increasing component has to be discarded. With the object nearby, however, matching of the boundary conditions requires the other component as well. As a result, one obtains exponentially increasing evanescent waves inside the negative-index material. Is there an energy gain connected with this? No, energy transport is only facilitated by propagating waves, i.e., for nonvanishing real part of the wave vector $\vec{k}$. Therefore, decaying evanescent waves are not connected with energy loss, and, similarly, increasing evanescent waves are not connected with gain. The bottom line is that all partial waves-for all spatial frequencies of the object-arrive in the image plane with exactly the original amplitude. Also, all optical path lengths are the same and zero, as the geometrical paths in vacuum (with $n=+1$ ) equal those in the negative-index material (with $n=-1$ ). Thus the resolution of the "perfect lens" is $\Delta x=0$, even in the far field, i.e., for $d \gg \lambda$, which has to be compared with the diffraction-limited resolution limit of a usual far-field lens of $\Delta x=\lambda / 2$.

It is important to note, however, that any tiny deviation from the singular limit of a "perfect lens", i.e., from a real refractive index of $n(\omega)=-1$, immediately limits the resolution $\Delta x$ to a finite value [437]. For simplicity, we only consider a two-dimensional geometry with $k_{y}=0$. Before reproducing exact analytic results under these conditions, we first give a simple heuristic argument for the overall structure: To obtain "super-resolution", the amplitudes of those partial waves with large spatial frequencies $k_{x}$ parallel to the slab are most important. With the dispersion relation of light, $k_{x}^{2}+k_{z}^{2}=\omega^{2} / c_{0}^{2}$, and for large spatial frequencies $k_{x}^{2} \gg \omega^{2} / c_{0}^{2}$, we obtain $k_{z}^{2} \approx-k_{x}^{2}$. Thus, the wave-vector component normal to the slab $k_{z}$ is given by $k_{z} \approx \mathrm{i} k_{x}$. The partial waves acquire a phase factor $\propto \exp \left(\mathrm{i} k_{z} d\right) \propto \exp \left(-k_{x} d\right)$ during propagation over distance $d$ in the "lens". If the proportionality factor does not balance this amplitude factor exactly-which is the case for a "perfect lens"- the exponential factor and hence the exponent need to have a certain finite value, i.e., $\exp \left(-k_{x} d\right) \approx$ const., thus $k_{x} d \approx$ const. We see that the spatial resolution $\Delta x \propto k_{x}^{-1} \propto d$ is proportional to the thickness of the slab $d=a+a^{\prime}$ : Large "focal lengths", equivalent to thick lenses, deteriorate the resolution.

With regard to exact results, we first consider a real refractive index given by

$$
n(\omega)=-1+\Delta n
$$

with $|\Delta n| \ll 1$. For the special symmetric "focusing" configuration $a=a^{\prime}=d / 2$, one obtains the strict result for the resolution from the exact two-dimensional Greens functions $[438,439]$

$$
\Delta x=-\frac{2 \pi d}{\ln (|\Delta n|)} .
$$

This expression deserves two numeric examples: Consider a slab 10 times the wavelength of light, i.e., $d=a+a^{\prime}=10 \lambda$. To obtain a resolution of $\Delta x=\lambda / 10$, this requires $|\Delta n|=\exp (-2 \pi 100) \approx 10^{-273}$ —a truly demanding level of control! This example implies that far-field imaging is totally out of reach-the "perfect lens" is definitely "near-sighted". For near-field imaging, however, things look more favorable. If, for example, we have $d=\lambda / 10$ (we will see below that this thickness corresponds to about one lattice constant of the metamaterial), and again asking for $\Delta x=\lambda / 10$, we merely need $|\Delta n|=\exp (-2 \pi) \approx 0.002$, which is still demanding but somewhat more reasonable experimentally.

Next, we consider the unavoidable finite imaginary parts of permittivity and permeability, i.e.,

$$
\epsilon(\omega)=\mu(\omega)=-1+\mathrm{i} \delta
$$

with $\delta \geqslant 0$ and $\delta \ll 1$. A rigorous calculation [440] (also see [441]) for two dimensions and (again) for the special symmetric "focusing" configuration $a=a^{\prime}=d / 2$ gives

$$
d=2 \Delta x \frac{\ln \left[\left(4\left(\xi^{2} \frac{\lambda^{2}}{(\Delta x)^{2}}-1\right)\right) /\left(\delta+\left(2 \xi^{2} \frac{\lambda^{2}}{(\Delta x)^{2}}-1\right) \delta\right)\right]}{4 \pi \sqrt{\xi^{2}-\frac{(\Delta x)^{2}}{\lambda^{2}}}} .
$$


a

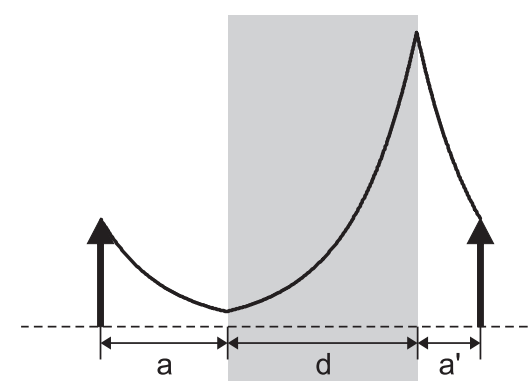

b

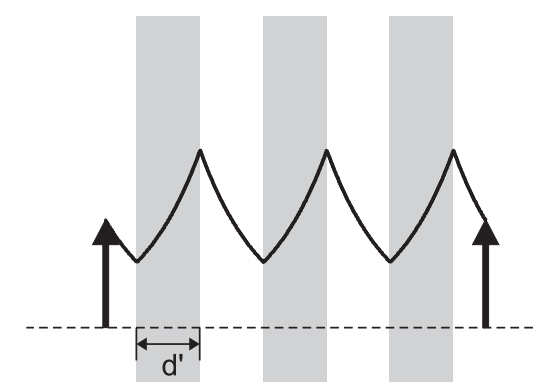

Fig. 51. (a) Simplified scheme of the evanescent fields of a "perfect lens" with thickness $d$, imaging a subwavelength object from the LHS to the RHS (compare with Fig. 50). Here, the resolution is given by $\Delta x \propto d$. (b) Corresponding scheme for a distributed "perfect lens", comprising individual slabs of thickness $d^{\prime} \ll d$. The resolution of this arrangement is given by $\Delta x \propto d^{\prime}$.

Here, we have introduced the numerical factor $\xi \approx 0.6$, which is the solution of the transcendental equation $\operatorname{sinc}(\pi \xi)=$ $1 / 2$. While this implicit expression cannot be solved exactly for $\Delta x$, it is apparent that the general structure is similar to the above case, the "perfect lens" is "near-sighted" indeed.

Example 6. If one is interested in high-resolution imaging over large distances, a stack of thin "almost perfect lenses" comes to the rescue. Such a distributed "almost perfect lens" [437,442] is illustrated in Fig. 51. According to Example 5 , the resolution $\Delta x$ of each individual thin "almost perfect lens" can be much better than the wavelength of light (see (5.141)). For a stack of usual lenses, each with resolution $\Delta x=\lambda / 2$, the resulting resolution would still be $\Delta x=\lambda / 2$. In analogy, the resolution of the stack of "perfect lenses" is no longer proportional to the overall thickness of the stack (as would be the case for a single thick "almost perfect lens"). The drawback of this arrangement, however, is that the object on the LHS and the image on the RHS have to be very close to the surfaces of the distributed "almost perfect lens". It has been predicted [437] that this concept also works if the individual slabs in Fig. 51(b) are made from a material with $\epsilon(\omega) \approx-1$ and $\mu(\omega)=+1$ (rather than $n(\omega) \approx-1$ ). A Drude metal at frequency $\omega=\omega_{\text {pl }} / \sqrt{2}$ would work. Corresponding imaging experiments at $365 \mathrm{~nm}$ wavelength using single silver layers with $d=35-50 \mathrm{~nm}$ (see Fig. 51(a)) have recently been presented in Refs. [443,444].

Example 7. Consider a one-dimensional periodic stack (a photonic crystal) with alternating quarter-wave layers of a usual dielectric material (e.g., $\epsilon=\mu=1)$ and a negative-index metamaterial $(\epsilon<0$ and $\mu<0)$. Material dispersion is neglected. It has been shown theoretically [445] that such a one-dimensional stack can exhibit a three-dimensional photonic band gap for one polarization (either TE or TM). For a periodic sequence of three (rather than two) different materials, even a complete three-dimensional photonic band gap can result [445], i.e., the 3D photon density of states is zero in a finite frequency interval. Thus, spontaneous emission would be totally suppressed for a dipole within the stack. The key for understanding this highly surprising result is that waveguiding within the layer planes can be suppressed when using negative-index materials [445]. In the same frequency region, usual Bragg reflection serves to suppress wave propagation perpendicular to the layers.

Yet further examples for the unusual properties of negative-index materials, like reverse Doppler shift, reverse Cherenkov radiation or reverse light pressure have already been discussed in Veselago's pioneering work from 1968 [49]. Using curved surfaces, also magnifying and demagnifying "perfect lenses" can be constructed [49]—again with sub-wavelength imaging capability [446]. Furthermore, a theorem has been proven [447] which states that the wavefront distortions due to an inhomogeneous medium, in which the refractive index varies in a direction perpendicular to the propagation direction, can be compensated by a medium having the identical variation in index profile, but with opposite sign. The linear optics of metamaterials also conceptually allows for making objects invisible by cloaking [448-450]. Quantum optical aspects such as, e.g., modified spontaneous emission of a two-level atom inside a negative-index material or in the close vicinity to a magnetodielectric material are discussed in Refs. [451,452] (using the Markov approximation); the theory of nonlinear optics of negative-index metamaterials, for example, in Refs. [453-458]. 
Negative-index materials can partly be mapped onto equivalent-circuit models [459]. In the microwave regime, such circuits can actually be realized by one-dimensional transmission lines [460,461] (also see Ref. [462]).

Finally, we discuss two remarks (that sometimes give rise to confusion) in the famous textbook by Landau and Lifshits on the electrodynamics of continuous media [463] concerning the magnetic permeability $\mu(\omega)$. First, Ref. [463] states at one place that the magnetic permeability has no meaning at optical frequencies, thus $\mu(\omega)=1$ in optics (for normal atoms and crystals). Indeed, if one uses the static definition of the magnetization (based on the normal current density vector $\vec{j}$ only) inside the optical regime, problems can arise because the "atomic" displacement current density $\dot{\vec{D}}$ can become comparable in magnitude to $\vec{j}$. For many (but not all) metamaterials to be discussed below, the displacement current is still much smaller than the normal current. This is, e.g., true for the split-ring resonator, the cut-wire pairs are an exception (see below). Generally, however, one simply must not use the magneto-static definition of the magnetization at optical frequencies. Note that the retrieval of $\epsilon(\omega)$ and $\mu(\omega)$ to be discussed below does not imply the static definition and elegantly circumvents this problem altogether.

Second, the description of an isotropic (meta)material in terms of $\epsilon(\omega)$ and $\mu(\omega)$ may be valid, but it is not unique. Indeed, it has already been pointed out in Ref. [463] that, alternatively, one can set $\tilde{\mu}=1$ and describe the (meta)material response in terms of spatial dispersion, i.e., via a wave-vector dependence of the electric permittivity. The strategy for deriving the connection between the two descriptions is to start by eliminating $\vec{D}$ and $\vec{H}$ from the Maxwell equations (2.1) and (2.2). Next, we are free to redefine them. We start with the known form for the wave equation in a linear homogeneous medium

$$
\Delta \vec{E}-\frac{1}{c_{0}^{2}} \frac{\partial^{2} \vec{E}}{\partial t^{2}}=+\mu_{0} \frac{\partial^{2} \vec{P}}{\partial t^{2}}+\mu_{0} \frac{\partial}{\partial t}(\vec{\nabla} \times \vec{M}) .
$$

After Fourier transformation and upon inserting for the polarization $\vec{P}=\epsilon_{0}(\epsilon(\omega)-1) \vec{E}$ and the magnetization

$$
\vec{M}=\frac{1}{\mu_{0}}\left(1-\frac{1}{\mu(\omega)}\right) \vec{B}
$$

we obtain

$$
-k^{2} \vec{E}+\frac{\omega^{2}}{c_{0}^{2}} \vec{E}=-\mu_{0} \epsilon_{0}(\epsilon(\omega)-1) \omega^{2} \vec{E}+\left(1-\frac{1}{\mu(\omega)}\right) \omega(\vec{k} \times \vec{B}) .
$$

Next, we redefine $\overrightarrow{\tilde{D}}=\epsilon_{0} \tilde{\epsilon} \vec{E}$ and $\vec{B}=\mu_{0} \tilde{\mu} \overrightarrow{\tilde{H}}$ with $\tilde{\mu}=1$. The induction law becomes

$$
\mathrm{i} \frac{1}{\mu_{0}} \vec{k} \times \vec{B}=-\mathrm{i} \epsilon_{0} \tilde{\epsilon} \omega \vec{E}
$$

Inserting (5.147) on the RHS of (5.146) and solving for $\tilde{\epsilon}$ leads to [455,463]

$$
\tilde{\epsilon}=\tilde{\epsilon}(\omega, k)=\epsilon(\omega)+\frac{k^{2} c_{0}^{2}}{\omega^{2}}\left(1-\frac{1}{\mu(\omega)}\right),
$$

where we have introduced the dispersion relation

$$
\frac{\omega^{2}}{k^{2}}=\frac{c_{0}^{2}}{\tilde{\epsilon}(\omega, k)}=\frac{c_{0}^{2}}{\tilde{n}^{2}(\omega, k)}
$$

with the refractive index $\tilde{n}(\omega, k) \neq n(\omega)$. Generally, $\tilde{n}(\omega, k)$ must, e.g., not be used in Snell's law. In real space, the vectors $\overrightarrow{\tilde{H}}(\vec{r})$ and $\vec{B}(\vec{r})$ are parallel by construction, whereas the vectors $\overrightarrow{\tilde{D}}(\vec{r})$ and $\vec{E}(\vec{r})$ are connected via a spatial convolution integral due to the $k$-dependence of the permittivity. Remember that spatial dispersion of the permittivity also occurs for electric quadrupole moments, indicating that magnetic dipoles and electric quadrupoles are closely related at optical frequencies [455].

Ultimately, it is a matter of taste which choice is preferred. In what follows, we continue with a description in terms of $\epsilon(\omega)$ and $\mu(\omega)$ rather than $\tilde{\epsilon}(\omega, k)$ and $\tilde{\mu}=1$. 


\subsubsection{Magnetic metamaterials}

In the examples of the preceding subsection we have seen that a variety of interesting phenomena arises for $\epsilon(\omega) \neq 1$ and $\mu(\omega) \neq 1$, especially for simultaneous $\operatorname{Re}(\epsilon(\omega))<0$ and $\operatorname{Re}(\mu(\omega))<0$ ("doubly negative materials"). Actually, getting any magnetic response at all is unusual as practically all naturally occurring materials have $\mu(\omega)=1$ at optical frequencies, whereas a negative permittivity occurs in any metal below the plasma frequency. A magnetic permeability $\operatorname{Re}(\mu(\omega))=\operatorname{Re}\left(1+\chi_{\mathrm{m}}(\omega)\right)<0$ arises from a magnetic susceptibility with $\operatorname{Re}\left(\chi_{\mathrm{m}}(\omega)\right)<-1$. This means that the magnetic dipole density (or magnetization) vector $\vec{M}$, i.e., magnetic dipoles per volume, needs to be sufficiently large in modulus and must be directed opposite to the external magnetic field.

Suppose that we have designed and realized a structure for which we anticipate a negative real part of the permeability. How can we verify or falsify this suspicion? Basically, the only observables are (angular-resolved) transmittance and reflectance spectra, at best comprising phase information. Equivalently, phase-dependent experiments in the time domain are possible. For a negative-index metamaterial, one obviously expects a negative phase difference $\varphi(\omega)$ connected with propagation through the material of thickness $d$. For a negative- $\mu$ but positive- $\epsilon$ structure, the phase would be positive. To "retrieve" the dependencies $\epsilon(\omega)$ and $\mu(\omega)$ for a metamaterial in the sense defined above, the following procedure [430,431] has been used quite frequently. It is based on the above equations (5.119) and (5.120), which imply a homogeneous and isotropic material, and on measured or calculated field transmittance and field reflectance spectra. When inverting these equations to derive the permittivity $\epsilon(\omega)$ and the permeability $\mu(\omega)$, one faces the problem that the solution is generally not unique because of the different branches of the inverse trigonometric functions involved (see Fig. 52). This problem is especially prominent for thick samples, where the phase $\varphi$ is much larger than $2 \pi$. To chose the correct branch, the following additional requirements can be used:

(i) For a passive medium, the imaginary part of the retrieved refractive index cannot be negative, i.e., $\operatorname{Im}(n(\omega)) \geqslant 0$. Otherwise, gain would result, which is in conflict with energy conservation.

(ii) The real part of the impedance has to be positive, i.e., $\operatorname{Re}(Z(\omega))>0$.

(iii) We expect the permittivity and the permeability to be continuous smooth functions of frequency, i.e., we neither expect discontinuities in these functions nor in their spectral derivatives.

At low frequencies $\omega$, the acquired phase $\varphi(\omega)=\left(\omega / c_{0}\right) n(\omega) d$ becomes smaller than $2 \pi$, hence only a single unique solution results. This, together with condition (iii), already sorts out most branches. Usually, if condition (ii) is additionally fulfilled, (i) results automatically. The dependences $\epsilon(\omega)$ and $\mu(\omega)$ inserted into Eqs. (5.119) and (5.120) deliver strictly the original reflectance and transmittance spectra. There are, however, subtleties. Sometimes, the choice of the thickness $d$ of a complicated nanostructure can be ambiguous, especially for thin structures, e.g., structures containing only a single lattice constant of a metamaterial. Intuitively, surface effects dominate in this case, whereas $\epsilon(\omega)$ and $\mu(\omega)$ are "bulk" properties. As a test, the thickness $d$ can be varied. If, for example, the thickness of a structure is doubled, $t(\omega)$ and $r(\omega)$ are reacquired, and $d$ is replaced by $2 d$, the same $\epsilon(\omega)$ and $\mu(\omega)$ should result from the retrieval. Furthermore, one needs to make sure that the metamaterial assumption underlying the procedure is not violated. For example, consider some structure with lattice constant $a$. For any given frequency $\omega$, the associated wavelength $\lambda$ given by $\lambda=2 \pi c_{0} /(|\operatorname{Re}(n(\omega))| \omega)$ has to fulfill the condition $\lambda / a \gg 1$ or at least $\lambda / a>2(\lambda / a=2$ is the Bragg condition), equivalent to the condition for the retrieved refractive index $n(\omega)$

$$
|\operatorname{Re}(n(\omega))|<\frac{\pi c_{0}}{\omega a} .
$$

This inequality corresponds to the area under a hyperbola versus frequency $\omega$. This tends to become a problem for a large modulus of the real part of the refractive index, i.e., close to strong resonances, especially for high-frequency resonances. To further test the validity of the metamaterial assumption, it can be helpful to vary the "lattice constant" $a$ of a structure while keeping the "atoms" constant in shape and size. True metamaterial resonances will not shift significantly in frequency, whereas resonances related to Bragg diffraction usually do.

We briefly note that if numerically computed or experimentally measured data are introduced into the retrieval procedure (rather than the fictitious analytical data in Fig. 52), the noise on these data can complicate the choice of the correct branch.

Generally, resonances as well as "anti-resonances" can result from the retrieval of metamaterial parameters. Here, the notion "anti-resonance" refers to a resonance that is phase shifted by $\pi$. Mathematically, for the example of Lorentz-oscillator responses, this corresponds to $\omega_{\mathrm{pl}, \mathrm{e}}^{2}<0$ for an electric resonance in (5.131) and to $f \omega^{2}<0$ for a magnetic resonance in (5.132). Thus, in the resonance, the corresponding imaginary parts $\epsilon^{\prime \prime}(\omega)$ and $\mu^{\prime \prime}(\omega)$ become 
a

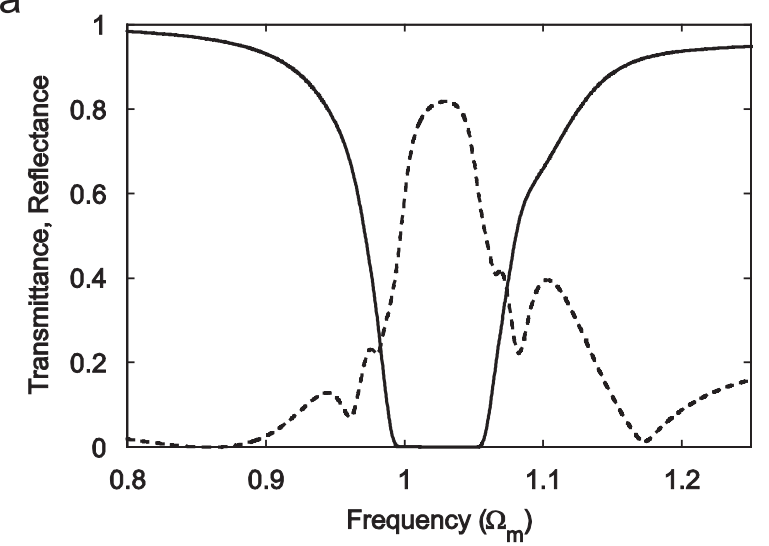

C

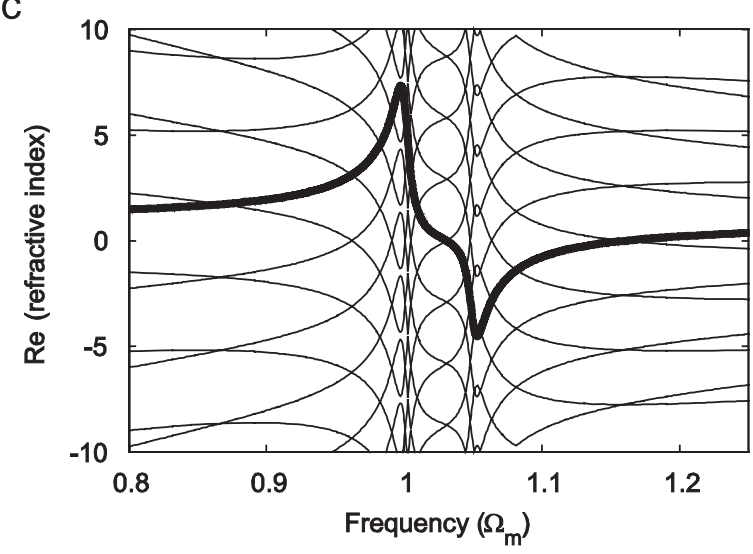

b

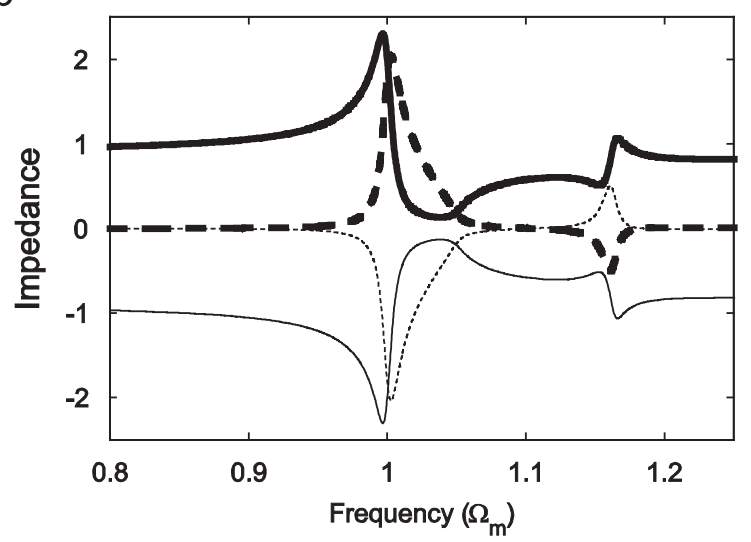

d

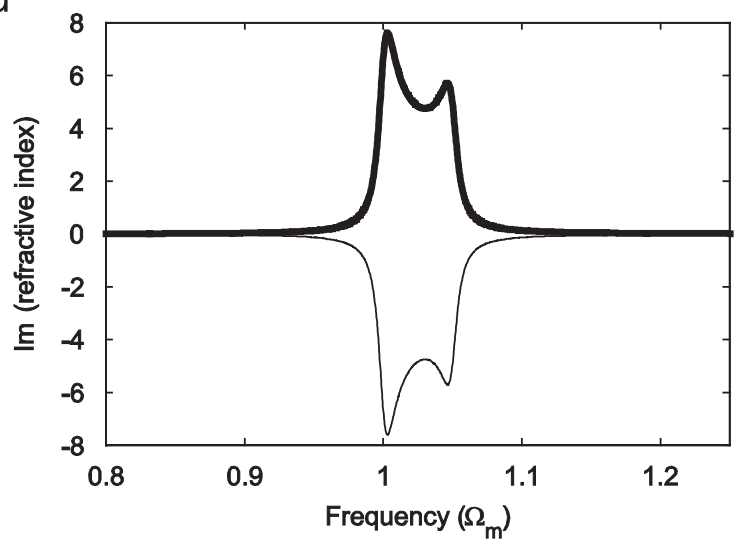

Fig. 52. Illustration of the retrieval procedure for an effective medium with a thickness $d$ equal to one-third of the magnetic resonance wavelength. The other parameters correspond to those of Fig. 46. (a) Assumed transmittance (solid curve) and reflectance (dashed curve) spectra are shown. The various mathematically possible branches for $z(\omega), \operatorname{Re}(n(\omega))$ and $\operatorname{Im}(n(\omega))$ are depicted in (b), (c) and (d), respectively. The branches highlighted by the thick curves are selected by applying the conditions (ii) and (iii) discussed in the text, (iii) is then fulfilled automatically. Solid and dashed curves in (b) correspond to $\operatorname{Re}(Z(\omega))$ and $\operatorname{Im}(Z(\omega))$, respectively.

negative. This does not necessarily violate energy conservation, which only requires that $\operatorname{Im}(n(\omega)) \geqslant 0$ for a passive medium. An antiresonance in $\epsilon(\omega)$ would, for example, violate causality if, at the same time, $\mu(\omega)=1$ holds. The negative imaginary part does not violate causality either: For a dielectric Lorentz oscillator response it just means that one replaces the driving force on the RHS of Newton's second law by its negative, i.e., $\vec{F} \rightarrow-\vec{F}$. This sign flip translates into a change of sign of the displacement and of the optical polarization, $\vec{P} \rightarrow-\vec{P}$, and hence of the electric susceptibility $\chi(\omega) \rightarrow-\chi(\omega)$, thus $\epsilon(\omega)=1+\chi(\omega) \rightarrow \epsilon(\omega)=1-\chi(\omega)$. For the imaginary parts this means that $\operatorname{Im}(\epsilon(\omega))=\operatorname{Im}(\chi(\omega))=\epsilon^{\prime \prime}(\omega) \rightarrow-\epsilon^{\prime \prime}(\omega)$ and similarly $\mu^{\prime \prime}(\omega) \rightarrow-\mu^{\prime \prime}(\omega)$. Consequently, the real-time response after, e.g., an impulsive excitation is a usual single-side exponential decay multiplied by an oscillatory function-but upside down. Equivalently, in the frequency domain, the poles of $\epsilon(\omega)$ and $\mu(\omega)$, respectively, still lie in the lower part of the complex $\omega$-plane, namely where $\Omega_{\mathrm{e}, \mathrm{m}}^{2}-\omega^{2}-\mathrm{i} \gamma_{\mathrm{e}, \mathrm{m}} \omega=0$. In other words: The Kramers-Kronig relations are still fulfilled. Could there be a problem with the electromagnetic energy density for anti-resonances? The general Abraham-Lorentz form for the electromagnetic energy density $u(\omega)$ [463] contains spectral derivatives. For $\omega=0$, this simplifies to the well-known static expression

$$
u(0)=\frac{1}{2}(\vec{D} \vec{E}+\vec{H} \vec{B})=\frac{1}{2}\left(\epsilon_{0} \epsilon(0) \vec{E}^{2}+\mu_{0} \mu(0) \vec{H}^{2}\right) .
$$

Remember that in the true static case $(\omega=0)$, permittivity and permeability are real and that electric and magnetic fields are no longer coupled. This is in sharp contrast to the limit $\omega \rightarrow 0$. Thus, $\epsilon(0)<0$ leads to an unphysical behavior, 
as the electric energy density becomes smaller as the electric-field strength increases. The same holds for $\mu(0)<0$ in magnetostatics. Thus, the retrieval needs to fulfill the two conditions $\epsilon(0) \geqslant 0$ and $\mu(0) \geqslant 0$. This effectively limits the oscillator strength of anti-resonances. For the specific example of undamped Lorentz responses this immediately leads to the conditions $\left|\omega_{\mathrm{pl}, \mathrm{e}}\right|<\Omega_{\mathrm{e}}$ in (5.131) and $f<1$ in (5.132). In contrast, at finite frequencies $\omega$, the finite-frequency energy density [463] can remain positive even for $\operatorname{Re}(\epsilon(\omega))<0$ and/or $\operatorname{Re}(\mu(\omega))<0$. The important bottom line of this discussion on resonances and anti-resonances is that the permittivity $\epsilon(\omega)$ and the permeability $\mu(\omega)$ are no longer independent quantities. Indeed, we will see in examples below that a resonance in $\mu(\omega)$ is accompanied by a weaker anti-resonance in $\epsilon(\omega)$ and, vice versa, a resonance in $\epsilon(\omega)$ is accompanied by a weaker anti-resonance in $\mu(\omega)$.

Magnetic-atom design 1: Let us now address the question how magnetic dipoles responding at optical frequencies can be achieved. It is well known from basic magnetostatics that a magnetic-dipole moment can be realized by the circulating ring current of a microscopic coil, which leads to an individual magnetic moment given by the product of current and area of the coil. This dipole-moment vector is directed perpendicular to the plane of the coil. If such a coil is combined with a plate capacitor, one expects an increased current at a finite-frequency resonance, hence, an increased magnetic-dipole moment. Thus, a popular design for magnetic "atoms" is to mimic a usual $L C$-circuit, consisting of a plate capacitor with capacitance $C$ and a magnetic coil with inductance $L$, on a scale much smaller than the relevant wavelength of light.

Fig. 53 shows the analogy of a conventional $L C$ circuit and a metallic split-ring resonator (SRR) on a dielectric surface. The RHS shows an electron micrograph of a single gold SRR fabricated by standard electron-beam lithography. The name "split-ring resonator" goes back to the 1981 work of Hardy and Whitehead [464] and that of Pendry from 1999 $[465,466]$. This name shall be employed below. However, SRR have also previously been discussed under the names "slotted-tube resonator" in 1977 [467] in the context of nuclear magnetic resonance (NMR) and "loop-gap resonator" in 1996 [468].

The position of the anticipated $L C$-resonance frequency can be estimated by the following crude approach: Suppose that we can describe the capacitance by the usual textbook formula for a large capacitor with nearby plates $(C \propto$ plate area/distance) and the inductance by the formula for a "long" coil with $N$ windings for $N=1$ ( $L \propto$ coil area/length). Using the nomenclature of Fig. 53(B), i.e., the width of the metal $w$, the gap of the capacitor $d$, the metal thickness $t$, and the width of the coil $l$, we get

$$
C=\epsilon_{0} \epsilon_{C} \frac{w t}{d} \propto \text { size }
$$

with the effective permittivity of the material in between the plates $\epsilon_{C}$, and

$$
L=\mu_{0} \frac{l^{2}}{t} \propto \text { size. }
$$
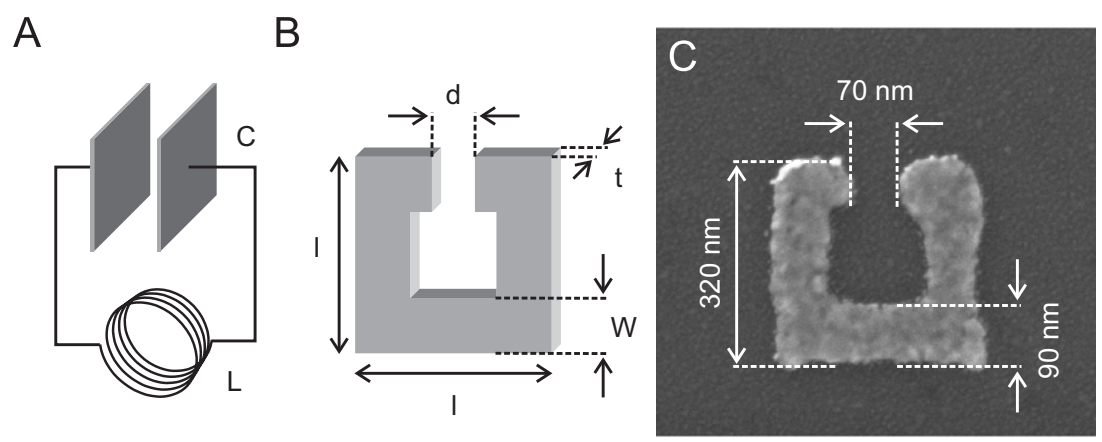

Fig. 53. Illustration of the analogy between a usual $L C$ circuit, A, and split-ring resonators (SRR), B. The electron micrograph in C shows an actually fabricated structure with gold SRR $(t=20 \mathrm{~nm})$ on a glass substrate. Taken from [469]. 
This leads to the eigenfrequency

$$
\omega_{L C}=\frac{1}{\sqrt{L C}}=\frac{1}{l} \frac{c_{0}}{\sqrt{\epsilon_{C}}} \sqrt{\frac{d}{w}} \propto \frac{1}{\text { size }},
$$

and to the $L C$-resonance wavelength

$$
\lambda_{L C}=\frac{2 \pi c_{0}}{\omega_{L C}}=l 2 \pi \sqrt{\epsilon_{C}} \sqrt{\frac{w}{d}} \propto \text { size. }
$$

For relevant parameters $\left(\epsilon_{C} \geqslant 1\right.$ and $\left.w \approx d\right)$, the prefactor is typically on the order of 10 , i.e., $\lambda_{L C} \approx 10 \times l$. Thus, it is possible to arrange these split-ring resonators in the form of an array such that the lattice constant $a$ is much smaller than the resonance wavelength, i.e., $a \ll \lambda_{L C}$. For example, for a telecommunication wavelength of $\lambda_{L C}=1.5 \mu \mathrm{m}$, the linear dimension of the coil would need to be on the order of $l=150 \mathrm{~nm}$, implying minimum feature sizes around $50 \mathrm{~nm}$ or yet smaller. Under these conditions, typical values for the capacitance and the inductance are $C \approx 1 \mathrm{aF}$ and $L \approx 1 \mathrm{pH}$, respectively. Third, the dielectric environment influences the resonance via $\epsilon_{C}$, which is, e.g., modified by the presence of a dielectric substrate. Fourth, if one closes the gap, i.e., in the limit $d \rightarrow 0$ or $C \rightarrow \infty$, the resonance wavelength goes to infinity, or equivalently, the resonance frequency $\omega_{L C}$ becomes zero.

What are the limits of size scaling according to (5.154) [470-473]? For an ideal metal, i.e., for an infinite electron density $n_{\mathrm{e}}$, hence an infinite metal plasma frequency $\omega_{\mathrm{pl}}$, a finite current $I$ flowing through the inductance is connected with zero electron velocity, hence with a vanishing electron kinetic energy. In contrast, for a real metal, i.e., for a finite electron density, the current is inherently connected with a finite electron velocity $v_{\mathrm{e}}$. Thus, one must not only provide the usual magnetic energy $\frac{1}{2} L I^{2}$ to support the current $I$, but additionally the total electron kinetic energy $N_{\mathrm{e}}\left(m_{\mathrm{e}} / 2\right) v_{\mathrm{e}}^{2}$ [142], where $N_{\mathrm{e}}=n_{\mathrm{e}} V$ is the number of electrons in the SRR contributing to the current. To conveniently incorporate this kinetic energy term into our electromagnetic formulation, we recast it into the form of an additional magnetic energy. Using $n_{\mathrm{e}} e v_{\mathrm{e}}=I /(w t)$ and the volume (=cross section times length) of the SRR wire $V=(w t)(4(l-w)-d)$, we obtain

$$
E_{\text {kin }}=N_{\mathrm{e}} \frac{m_{\mathrm{e}}}{2} v_{\mathrm{e}}^{2}=\frac{1}{2} L_{\mathrm{kin}} I^{2} .
$$

Here, we have introduced the "kinetic inductance"

$$
L_{\text {kin }}=\frac{m_{\mathrm{e}}}{n_{\mathrm{e}} e^{2}} \frac{4(l-w)-d}{w t} \propto \frac{1}{\operatorname{size}} .
$$

While the usual inductance $L$ is proportional to the SRR size (5.153), the kinetic inductance (5.157) scales inversely with size-provided that all SRR dimensions are scaled down simultaneously. Thus, the kinetic inductance is totally irrelevant for macroscopic coils but becomes dominant for microscopic inductances, i.e., when approaching optical frequencies. The kinetic inductance adds to the usual inductance, $L \rightarrow L+L_{\text {kin }}$ in (5.154), and we immediately obtain the modified scaling for the magnetic resonance frequency

$$
\omega_{L C} \propto \frac{1}{\sqrt{\text { size }^{2}+\text { const. }} .}
$$

Obviously, the magnetic resonance frequency is inversely proportional to size for large SRR, whereas it approaches a constant for small SRR. It is easy to show that this constant scales with the metal plasma frequency-as one would expect.

Can we obtain an explicit expression for the magnetic permeability $\mu(\omega)$ from our simple $L C$-circuit reasoning? We start by considering an excitation configuration where the electric-field component of the light cannot couple to the SRR (see below) and where the magnetic field is normal to the SRR plane. Under these conditions, the self-induction voltage of the inductance $L$ plus the voltage drop over the capacitance $C$ equals the voltage $U_{\text {ind }}$ induced by the external magnetic field, i.e., $U_{L}+U_{C}=U_{\text {ind }}$ or

$$
L \dot{I}+\frac{1}{C} \int \mathrm{d} t I=U_{\text {ind }}=-\dot{\phi}
$$


Again assuming a homogeneous magnetic field in the coil, we obtain the external magnetic flux $\phi=l^{2} \mu_{0} H$, with the external magnetic field $H=H_{0} \mathrm{e}^{-\mathrm{i} \omega t}+$ c.c. Taking the time derivative of (5.159) and dividing by $L$ yields

$$
\ddot{I}+\frac{1}{L C} I=\frac{1}{L} \dot{U}_{\text {ind }}=+\omega^{2} \frac{\mu_{0} l^{2}}{L} H_{0} \mathrm{e}^{-\mathrm{i} \omega t}+\text { c.c. }
$$

Upon inserting the obvious ansatz $I=I_{0} \mathrm{e}^{-\mathrm{i} \omega t}+$ c.c., we obtain the current $I$, the individual magnetic-dipole moment $l^{2} I$, and the magnetization $M=\left(N_{L C} / V\right) l^{2} I$. Here, we have introduced the number of $L C$ circuits $N_{L C}$ per volume $V$. Suppose that the lattice constant in the SRR plane is $a_{x y} \geqslant l$, and $a_{z} \geqslant t$ in the direction normal to the SRR. This leads to $N_{L C} / V=1 /\left(a_{x y}^{2} a_{z}\right)$. Finally inserting $M=\chi_{\mathrm{m}}(\omega) H, \mu(\omega)=1+\chi_{\mathrm{m}}(\omega)$, and (5.154) brings us to

$$
\mu(\omega)=1+\frac{f \omega^{2}}{\omega_{L C}^{2}-\omega^{2}} .
$$

Here, we have lumped the various prefactors into the dimensionless quantity $f$ with

$$
0 \leqslant f=\frac{l^{2} t}{a_{x y}^{2} a_{z}} \leqslant 1 .
$$

$f=1$ corresponds to nearest-neighbor SRR touching each other-obviously the ultimate upper bound for the accessible SRR density. Thus, we can interpret $f$ as a filling fraction. Roughly, (5.161) represents a Lorentz-oscillator resonance that we have already used above (see (5.132)). A subtle difference with respect to the usual Lorentz oscillator form is the $\propto \omega^{2}$ numerator, which leads to the asymptotics $\mu(0)=1$ and $\mu(\infty)=1-f$. Clearly, in the static limit, no current can be induced, so that $\mu(0)=1$. The limit $\mu(\infty)=1-f$ is a pathology of the model and would have to be replaced by $\mu(\infty)=1$ when accounting for the actual metal permittivity $\epsilon(\omega)$.

The permeability of a closed ring, ${ }^{8}$ i.e., the special case of $d \rightarrow 0 \Rightarrow C \rightarrow \infty \Rightarrow \omega_{L C} \rightarrow 0$ in (5.161), reduces to $\mu(\omega)=$ const. $=1-f \geqslant 0$. In other words, the split in the ring is essential for obtaining $\operatorname{Re}(\mu(\omega))<0$. For example, for $30 \%$ lateral spacing $\left(a_{x y}=1.3 \times l\right)$ and for a spacing in the vertical direction equal to the SRR thickness $\left(a_{z}=2 \times t\right)$, we obtain $f=0.30$ and $\mu=0.70$. Note, however, that we have tacitly neglected the interaction among the rings in our considerations leading to this conclusion. The assumption of noninteracting rings is justified for $f \ll 1$, but becomes questionable for $f \rightarrow 1$. What qualitative modifications are expected from the interaction of rings? The fringing field of any particular ring at the location of its in-plane neighbors is opposite to its own magnetic-dipole moment, hence parallel to the external magnetic field of the light. Thus, in-plane interaction tends to effectively reduce $\mu$. In contrast, interaction with rings from adjacent parallel planes tends to increase $\mu$. It is presently unclear, whether a particular arrangement of rings could allow for $\mu<0$ (also see Ref. [466]). Interaction similarly influences the behavior of split rings. We will twice come back to this aspect below.

So far in our discussion of SRR, we have neglected any damping, i.e., we have $\gamma_{\mathrm{m}}=0$. What mechanisms can generally influence the spectral width of the resonance in (5.161)? A first source of broadening is the finite conductivity $\sigma$ of the metal used for the SRR, which can be expressed as a finite Ohmic resistance $R$ in the $L C$ circuit. This leads to the extension of (5.160)

$$
\ddot{I}+\frac{R}{L} \dot{I}+\frac{1}{L C} I=\frac{1}{L} \dot{U}_{\text {ind }},
$$

where the ratio $R / L$ plays the role of the Stokes damping of an harmonic oscillator. By coefficient comparison with (5.132) (also see (5.160) and (5.161)) we obtain $\gamma_{\mathrm{m}}=R / L$. The total resistance $R$ of the SRR shown in Fig. 53 is given by the length of the metal wire divided by its cross section and its conductivity $\sigma$, i.e.,

$$
R=\frac{4(l-w)-d}{w t \sigma} \propto \frac{1}{\text { size }},
$$

which leads to the quality factor of the $L C$ resonance (within and outside of the size scaling regime)

$$
\frac{R / L}{\omega_{L C}}=\frac{\gamma_{\mathrm{m}}}{\omega_{L C}} \propto \frac{1}{\operatorname{size}} .
$$

\footnotetext{
${ }^{8}$ This limit does not contradict the discussion above because the case $\omega_{L C}=\omega=0$ is pathological as we have neglected damping so far.
} 
The bottom line of this simple reasoning is that we expect Ohmic damping to become a problem for very small SRRs, equivalent to large $L C$ frequencies. This trend is enhanced by the fact that the Drude model ac conductivity $\operatorname{Re}(\sigma(\omega))$ decreases with respect to the dc conductivity $\sigma=\operatorname{Re}(\sigma(0))$ via $\operatorname{Re}(\sigma(\omega))=\operatorname{Re}(\sigma(0))\left(1+\omega^{2} / \gamma^{2}\right)^{-1}$ as frequency increases. For example for the parameters of Fig. 53, with $\epsilon_{C}=1.5$ and the room temperature gold dc conductivity $\sigma=4.5 \times 10^{7}(\Omega \mathrm{m})^{-1}$ we obtain $R=10 \Omega, L=6.4 \mathrm{pH}, C=0.23 \mathrm{aF}, \omega_{L C}=2 \pi 100 \mathrm{THz}$, and $\gamma_{\mathrm{m}}=0.003 \omega_{L C}$.

Second, due to the close proximity of adjacent split-ring resonators, the degeneracy of the individual $L C$ resonances is lifted, which leads to a certain broadening of the resonance that depends on the lattice constant $a$. The underlying physics is related to the formation of magnon-like excitations [8] from individual magnetic moments due to local spins. In the tight-binding approximation, this leads to a cosine-shaped energy band, replacing the degenerate discrete energy levels. The inductive coupling between SRR can also give rise to a novel type of waves called magnetoinductive waves [474-476], which have been observed experimentally at $60 \mathrm{MHz}$ frequency [477] and at optical frequencies [478]. A third contribution to the width of the magnetic resonance is radiation damping arising from oscillating electric dipoles. This mechamism also increases with increasing frequency. A fourth broadening mechanism is inhomogeneous broadening [479] due to fabricational tolerances of the SRR: While there is no intrinsic frequency dependence, one has to push today's fabrication technology to its limits to achieve sufficiently small structures. This leads to an effective (extrinsic) increase of the influence of inhomogeneous broadening with increasing resonance frequency. Finally, a fifth contribution for metal SRR is Landau damping. Recall that Landau damping describes the decay of the collective plasmon-polariton excitation into single-particle excitations at large momenta [395]. It is not very important for the optical properties of bulk metals. For small metal nanoparticles, however, large momenta occur via the uncertainty principle. It has been suggested theoretically [480] that this mechanism becomes relevant for wave vectors of light $k>\omega / v_{\mathrm{F}}$, with the electron velocity at the Fermi surface $v_{\mathrm{F}}$. This translates into metal particle sizes $\Delta x \approx 2 \pi / k<\lambda v_{\mathrm{F}} / c_{0}$, and might be the ultimate limitation for the resolution of a "perfect lens" [480]. For example, with $v_{\mathrm{F}}=1.40 \times 10^{6} \mathrm{~m} / \mathrm{s}$ for $\mathrm{Au}\left(v_{\mathrm{F}}=1.39 \times 10^{6} \mathrm{~m} / \mathrm{s}\right.$ for $\left.\mathrm{Ag}\right), \Delta x$ is about $0.5 \%$ of the wavelength of light $\lambda$, for $\lambda=3 \mu \mathrm{m}$ we get $\Delta x=15 \mathrm{~nm}$ (cf. Fig. 53(C) and RHS column in Fig. 54). Conceptually, theory has to account for the wave-vector dependence of the metal dielectric function $\epsilon(\omega, k)$ ("spatial dispersion"), equivalent to considering a nonlocal optical response in real space. Such treatment has rarely been performed. Experimentally, this aspect could be quite important because one hardly ever gets perfectly smooth metal films. In typical evaporation processes, one rather gets a collection of more or less connected metal grains (see, e.g., Fig. 53(C)).

Historically, the first demonstration of negative-index materials was in 2001 at around $10 \mathrm{GHz}$ frequency or $3 \mathrm{~cm}$ wavelength [481], where the split-ring resonators can easily be fabricated on electronic circuit boards. The negative permittivity was achieved by additional metal stripes. In 2004 [482], $\operatorname{Re}(\mu(\omega))<0$ has been demonstrated at about $1 \mathrm{THz}$ frequency $(300 \mu \mathrm{m}$ wavelength) using standard microfabrication techniques for the SRR, Ref. [483] reviews this early microwave work. More recently, arrays of nanoscopic split-ring resonators have been fabricated [469] (see Fig. 53). Using the above formula (5.155), we estimate an $L C$ resonance wavelength of about $3 \mu \mathrm{m}$ ( $100 \mathrm{THz}$ frequency). A part of the spectroscopic results on such samples is shown in Fig. 54. Here, the $L C$ circuits are arranged in a square lattice on a glass substrate coated with a 5-nm thin film of indium-tin oxide. The latter prevents charging effects in the electron-beam writing process. The magnetic field vector of the incident light has a vanishing component normal to the coil for normal incidence conditions. Thus, coupling to the $L C$ resonance is only possible if the electric-field vector has a component normal to the plates of the capacitance [484], which corresponds to the left column of spectra in Fig. 54. Independent on the lattice constant, $a$, two distinct resonances are clearly visible. With increasing $a$, the resonances narrow to some extent because of the reduced interaction between the split-rings resonators, but their spectral position remains essentially unchanged as expected for the electric and magnetic resonant responses of split-ring resonators (SRR). The long-wavelength resonance around $3 \mu \mathrm{m}$ wavelength completely disappears if the electric-field vector is rotated by $90^{\circ}$ (right column of spectra in Fig. 54). This is expected for the $L C$ resonance.

To further strengthen the interpretation of the $3 \mu \mathrm{m}$ resonance in terms of an $L C$ resonance, corresponding spectra for closed rings rather than split rings are shown in the last row of Fig. 54(G and H). Indeed, the $3 \mu \mathrm{m}$ resonance does not occur for either linear polarization in this case, and the reflectance and transmittance spectra are nearly identical for the two polarizations, apart from minor deviations, due to imperfections in the nanofabrication process. The additional transmittance minimum between 1 and $2 \mu \mathrm{m}$ wavelength is due to the particle plasmon or Mie resonance (an electricdipole resonance) already discussed in Section 5.3. All features of the measured spectra are reproduced by numerical calculations using a three-dimensional finite-difference time-domain approach [469] (see Section 2.1.4.3). The field distributions (not shown) corresponding to the sample and excitation parameters of the $3-\mu \mathrm{m}$ resonance (Fig. 54(A)) 


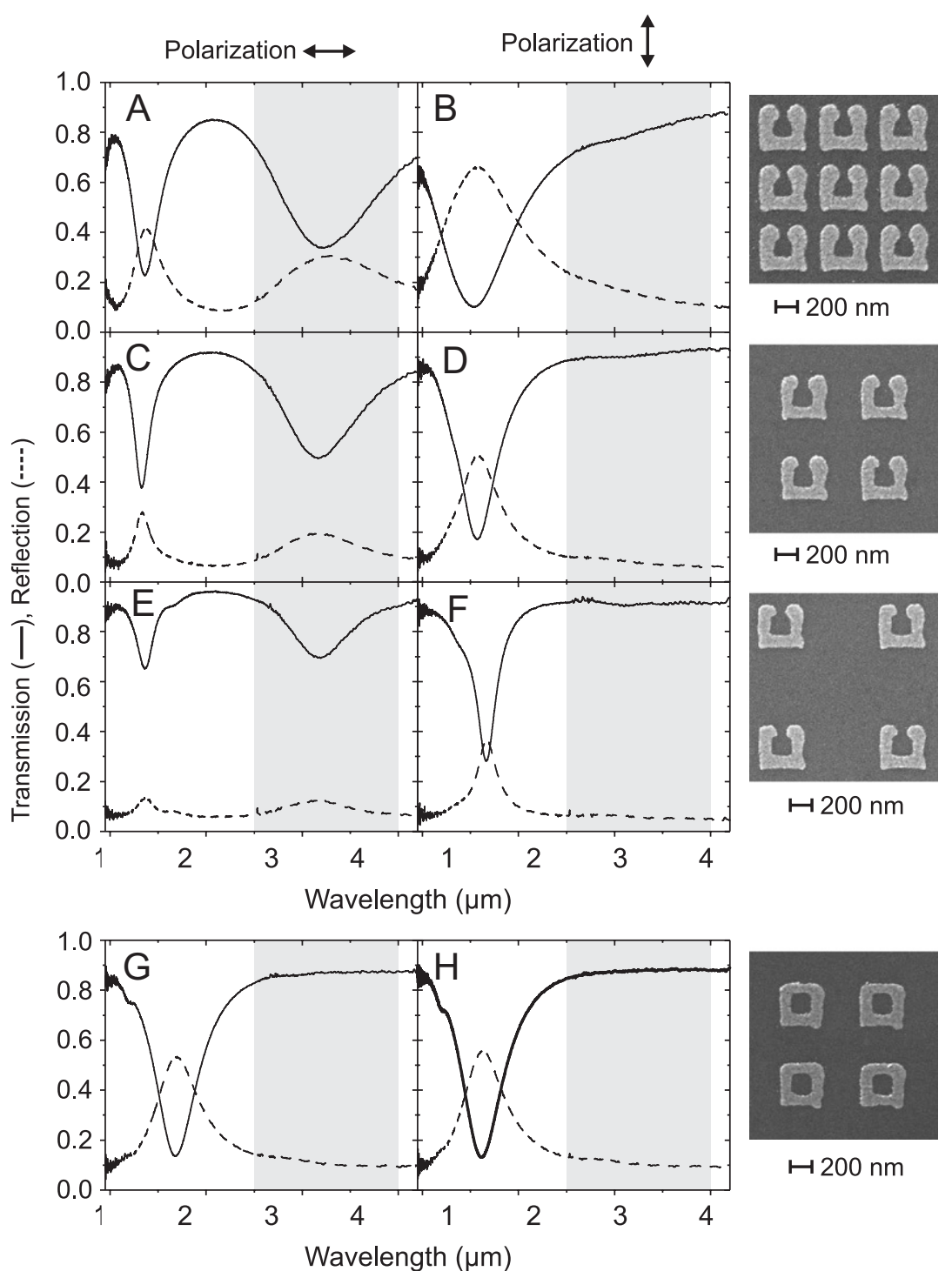

Fig. 54. Measured transmittance and reflectance spectra (normal incidence) of a magnetic metamaterial. In each row of this "matrix", an electron micrograph of the corresponding sample is shown on the RHS. The two polarization configurations are shown on top of the two columns. A, B (lattice constant $a=450 \mathrm{~nm}), \mathrm{C}, \mathrm{D}(a=600 \mathrm{~nm})$ and E, F $(a=900 \mathrm{~nm})$ correspond to nominally identical split-ring resonators, $\mathrm{G}$ and $\mathrm{H}(a=600 \mathrm{~nm})$ to corresponding closed rings. The combination of these spectra unambiguously shows that the resonance at about $3 \mu \mathrm{m}$ wavelength (highlighted by the gray areas) is the $L C$ resonance of the individual split-ring resonators. Taken from [469].

reveal a fairly homogeneous electric field between the plates of the capacitor. This a posteriori justifies the above use of the simple formula for the capacitance $C$ of the plate capacitor. Furthermore, one obtains a circulating electric field inside the metal ring, which leads to a circulating Ohmic current, hence to a magnetic-dipole moment perpendicular to the plane of the coil.

The permittivity $\epsilon(\omega)$ and the permeability $\mu(\omega)$ retrieved from the calculated transmittance and reflectance spectra (along the lines described above) are depicted in Fig. 55 for the two different polarization/propagation geometries illustrated by the insets. Parameters correspond to those of Fig. 54(A). Note that the resonance in $\mu(\omega)$ on the RHS is accompanied by an anti-resonance in $\epsilon(\omega)$ and, vice versa, on the LHS (see corresponding discussion above).

Magnetic-atom design 2: Broadly speaking, the individual SRR can be viewed as a metal particle, i.e., a square pad, a part of which has been cut out, leading to a certain notch depth. Thus, we expect that the magnetic resonance evolves 

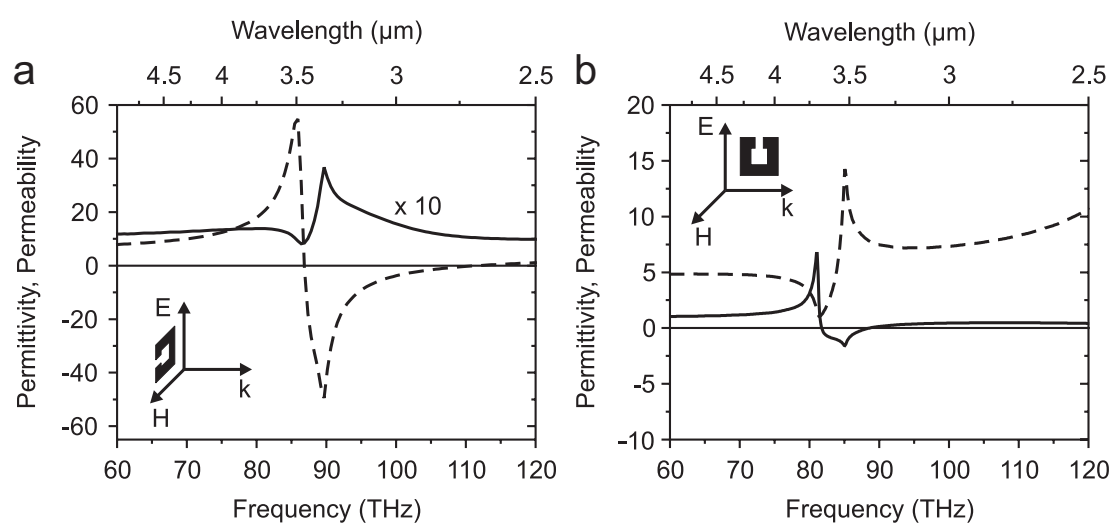

Fig. 55. Real part of the permittivity $\epsilon(\omega)$ (dashed curves) and permeability $\mu(\omega)$ (solid curves) as retrieved for two different geometries (a) and (b) for the sample parameters of Fig. 54(A). Taken from [469].
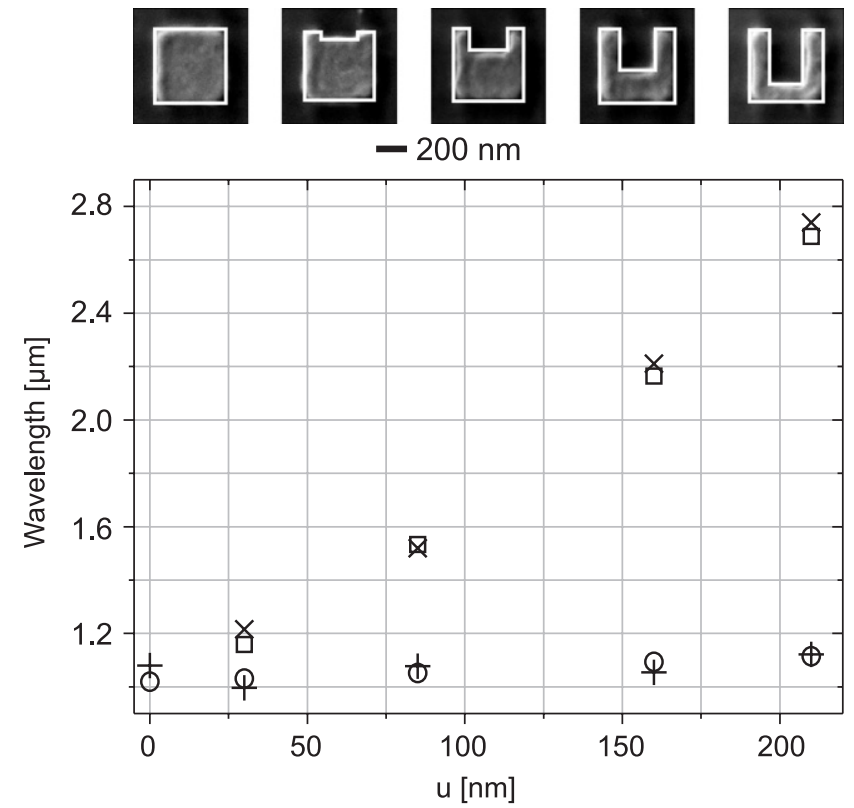

Fig. 56. Measured (crosses) and calculated (circles and squares) spectral positions of the long-wavelength magnetic resonance and the short-wavelength electric resonance as a function of the notch depth $u$ (from left to right: $u=0,30,85,160$, and $210 \mathrm{~nm}$ ), horizontal polarization. The corresponding five magnetic "atoms", which have been fabricated by focused-ion-beam milling, are depicted at the top. They are arranged in a two-dimensional square lattice with $a=450 \mathrm{~nm}$ on top of a glass substrate. Taken from Ref. [485].

out of the Mie resonance (see Section 5.3) of a square pad with increasing notch depth [485]. Experiments of this sort have been performed indeed. Fig. 56 shows the observed and calculated positions of the transmittance resonances. For a square-pad particle, the two Mie resonances for the two orthogonal linear polarizations are energetically degenerate. As soon as a notch as small as $30 \mathrm{~nm}$ in depth is introduced, the degeneracy is lifted and two distinct resonances are observed. For vertical polarization again only one resonance is observed which stays roughly constant in wavelength. In contrast, for horizontal polarization, two distinct resonances evolve. The spectral position of one of these coincides roughly with that for vertical polarization. The spectral position of the other one gradually shifts towards longer wavelengths (lower frequencies) with increasing notch depth. In agreement with our discussion above, this low-frequency resonance has a ring current, hence a magnetic-dipole moment connected to it. A detailed theoretical analysis [485] furthermore shows that the magnetic susceptibility increases in peak modulus as the notch depth increases. Only for the notch depths of 

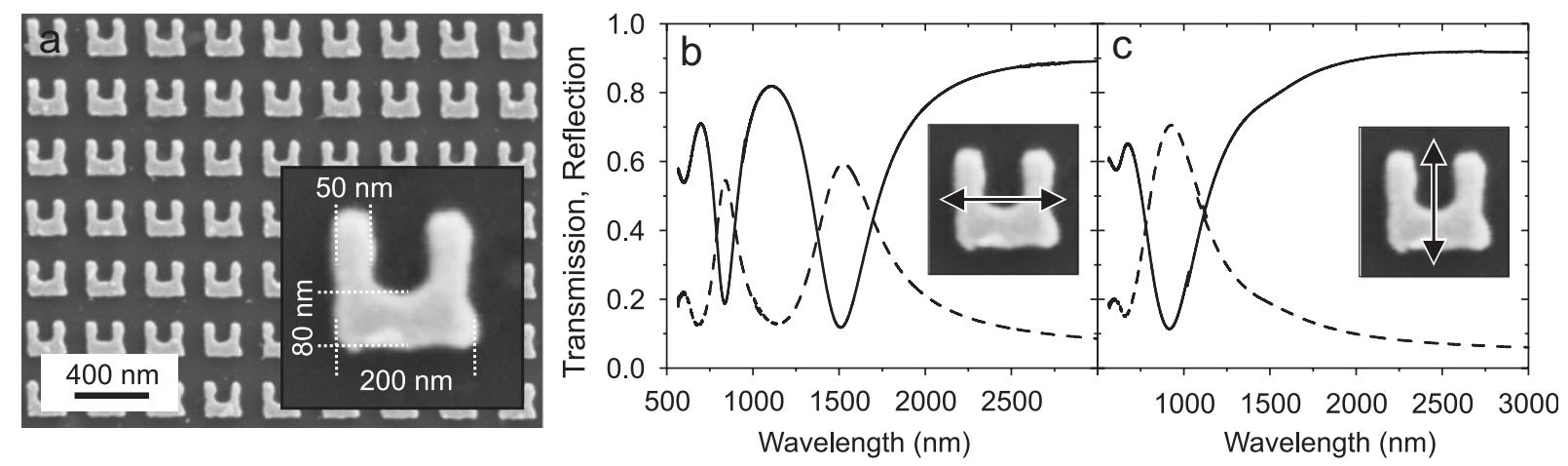

Fig. 57. Electron micrograph of a split-ring array with a total size of $(100 \mu \mathrm{m})^{2}$. The lower RHS inset shows the dimensions of an individual split ring. The corresponding measured normal-incidence transmittance and reflectance spectra for horizontal and vertical polarization are shown in (b) and (c), respectively. Taken from [488].

a

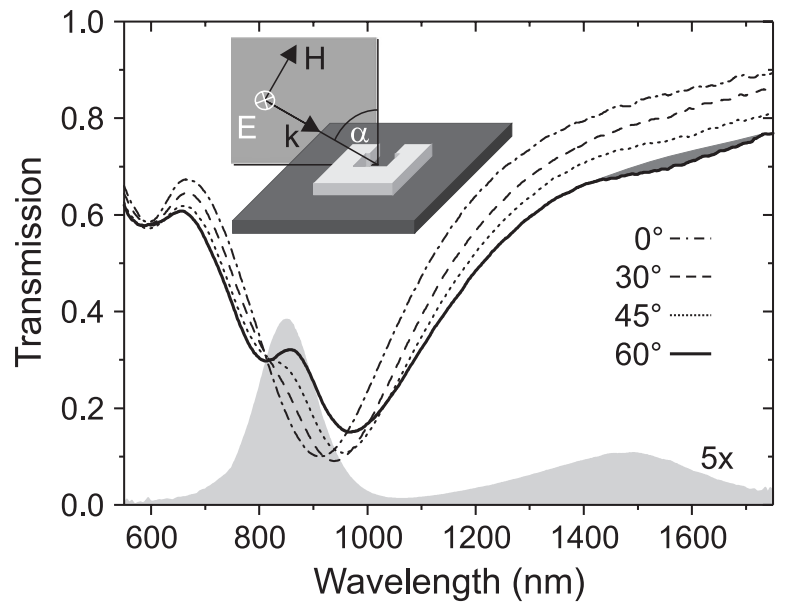

b

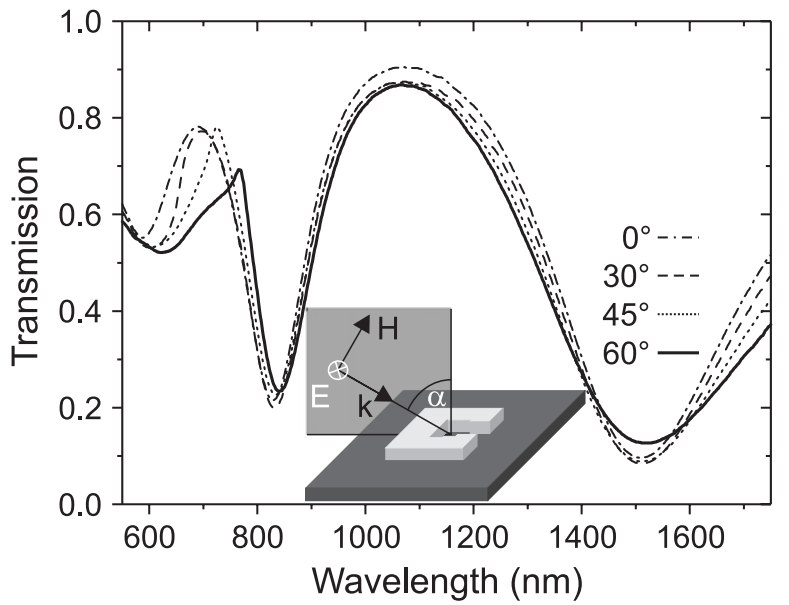

Fig. 58. Measured transmittance spectra taken for oblique incidence for the configurations shown as insets (where $\alpha=60^{\circ}$ ). In (a), coupling to the fundamental magnetic mode at 1.5 - $\mu \mathrm{m}$ wavelength is only possible via the magnetic-field component of the incident light, for (b), both electric and magnetic field can couple. Note the small but significant feature in (a) for $60^{\circ}$ around $1.5-\mu \mathrm{m}$ wavelength. The lower gray area in (a) is the transmittance into the linear polarization orthogonal to the incident one for $\alpha=60^{\circ}$. This observable can be viewed as a fingerprint of magnetic resonances under these conditions. Taken from [488].

160 and $210 \mathrm{~nm}$ in Fig. 56 does the magnetic permeability become negative (for a wave vector in the plane of the SRR). A further experimental study regarding the detailed influence of the SRR shape has recently been published in Ref. [486].

Similar "U"-shaped SRR structures [487] have also been fabricated via electron-beam lithography (Fig. 57), leading to an optical quality superior to that of the focused-ion-beam structures [488]. In such structures, also the coupling to the magnetic resonance via the magnetic-field component of the light can be evaluated for oblique incidence.

In Fig. 58(a), the electric component of the incident light cannot couple to the $L C$ circuit resonance for any angle. With increasing angle, however, the magnetic field acquires a component normal to the SRR plane. This component can induce a circulating electric current in the SRR coil via the induction law. This current again leads to a magnetic-dipole moment normal to the SRR plane, which can counteract the external magnetic field. The magnitude of this resonance (highlighted by the dark gray area around 1.5- $\mu \mathrm{m}$ wavelength) is indeed consistent with theory [488] (not depicted here) and leads to an effective negative magnetic permeability for propagation in the SRR plane and for a stack of SRR layers rather than just one layer considered here. This aspect has been verified explicitly by retrieving the effective 
permittivity and permeability from the calculated transmittance and reflectance spectra [430,431]. The shape of the retrieved magnetic permeability closely resembles that of the $3-\mu \mathrm{m}$ wavelength structures above [469]. It exhibits a negative permeability with a minimum value of $\operatorname{Re}(\mu)=-0.25$ at $1.67-\mu \mathrm{m}$ wavelength [488]. This value could be further improved by increasing the number of SRR per area (compare Fig. 57(a) and/or (5.161)), hence increasing the "oscillator strength" of the magnetic resonance.

Another striking feature of the spectra in Fig. 58(a) is that the 950-nm wavelength Mie resonance at normal incidence splits into two resonances for oblique incidence. This aspect is reproduced by numerical calculations [488]. Intuitively, it can be understood as follows: for normal incidence and horizontal incident polarization and for the frequencies of interest here, only the fundamental Mie resonance of the SRR bottom arm is excited. ${ }^{9}$ For normal incidence and vertical polarization, the two similarly shaped vertical SRR arms contribute. The latter are coupled via the SRR's bottom arm (and via the radiation field). As usual, the coupling of two degenerate modes leads to an avoided crossing with two new effective oscillation modes, a symmetric and an anti-symmetric one, which are frequency down-shifted and up-shifted as compared to the uncoupled resonances, respectively. The anti-symmetric mode cannot be excited at all for normal incidence as it has zero effective electric-dipole moment. The red-shifted symmetric mode can be excited. It even has a larger effective electric-dipole moment than a single arm. Indeed, the Mie resonance for vertical polarization is deeper and spectrally broader than for horizontal polarization in Fig. 54 and red shifted with respect to it. For finite angles of incidence, the phase fronts of the electric field are tilted with respect to the SRR plane. Thus, the vertical SRR arms are excited with a small but finite time delay, equivalent to a finite phase shift. This shift allows coupling to the anti-symmetric mode of the coupled system of the two vertical arms as well. In one half cycle of light, one gets a positive charge at the lower LHS corner of the SRR and a negative charge at the lower RHS corner, resulting in a compensating current in the horizontal bottom arm.

According to this reasoning for oblique incidence (e.g., $60^{\circ}$ ), we expect a circulating current component for wavelengths near the two magnetic resonances at $1.5 \mu \mathrm{m}$ and $800 \mathrm{~nm}$, respectively. Any circulating current is evidently connected with a current in the horizontal bottom arm of the SRR. According to the usual laws of a Hertz dipole, the corresponding charge oscillation in the bottom arm can radiate into the forward direction with an electric-field component orthogonal to the incident polarization. In other words for oblique incidence, the fingerprint of the magnetic resonances is a rotation of polarization. Such rotation is indeed unambiguously observed in the experiments (see gray area in Fig. 58(a)).

Alternatively, the short-wavelength $(\approx 800 \mathrm{~nm})$ magnetic resonance in Fig. 58 can be interpreted as a standing wave of the current on the SRR with two nodes along the perimeter of the SRR [489]. Naively, one would expect this resonance to be located at three times the fundamental magnetic-resonance frequency. The actual position strongly deviates from that expectation, e.g., due to the nonlinear surface-plasmon-polariton dispersion.

The results discussed so far have all been within the SRR size-scaling regime. SRR with $l \approx 100 \mathrm{~nm}$ side length lead to a measured fundamental magnetic-resonance frequency of $325 \mathrm{THz}(900 \mathrm{~nm}$ wavelength) [490]. This value approaches the numerically calculated maximum magnetic-resonance frequency of $373 \mathrm{THz}$ [490], i.e., further miniaturization would not significantly increase the magnetic-resonance frequency-in agreement with our qualitative discussion above (see (5.158)). For a detailed quantitative understanding, however, one should appreciate that the Drude model (5.92) is no longer an adequate description of the gold at this point and one should rather use the actual gold dielectric function (see Fig. 37) as input for the numerical calculations [490].

Early experiments on the nonlinear optics (i.e., second-harmonic generation) of magnetic metamaterials composed of SRR have been presented in Ref. [491].

Magnetic-atom design 3: The "U"-shaped structures have only been a minor variation of the scheme of split-ring resonators. Are there other, more distinctly different options? Fig. 59(a) illustrates the connection between SRR and cut-wire pairs [432,487,492-494]. On the LHS, a usual SRR is shown. Following our above reasoning in terms of an $L C$ circuit, opening up the slit in the SRR decreases the capacitance $C$, hence increases the $L C$ resonance frequency. Additionally opening the bottom arm of the " $U$ " leads to a second serial capacitance, further reducing the net capacitance in the circuit and an increasing of the resonance frequency. Further opening of the lower slit brings us to a pair of cut wires (wire pieces). The result of this transition is that the Ohmic currents in the horizontal arms on the LHS of Fig. 59(a) have been replaced by displacement currents on the RHS. On the one hand, we have increased the $L C$ resonance frequency

\footnotetext{
${ }^{9}$ Strictly speaking, of course, all resonances of the SRR are plasmonic resonances of the entire SRR. However, if a certain part of the SRR is predominantly involved for a certain resonance, it is meaningful to say that this resonance is associated with this part.
} 


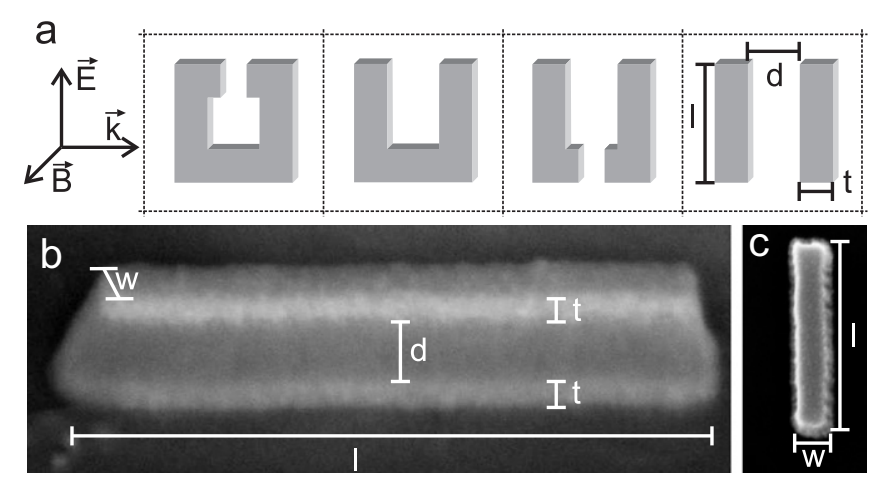

Fig. 59. (a) Scheme illustrating the "adiabatic" transition from split-ring resonators (LHS) to cut-wire pairs (RHS) as "magnetic atoms" of optical metamaterials. (b) Electron micrograph (oblique-incidence view) of an actual cut-wire pair with $w=150 \mathrm{~nm}, t=20 \mathrm{~nm}, d=80 \mathrm{~nm}, l=1000 \mathrm{~nm}$, (c) corresponding top view. Taken from [492].

for a given minimum feature size. This eases access to the (near-)visible regime at reduced fabricational effort. On the other hand, this increased resonance frequency at fixed lattice constant decreases the ratio between (resonance) wavelength, $\lambda$, and lattice constant, $a$, to about $\lambda / a \approx 2-3$. In the true metamaterial limit, one aims at $\lambda / a \gg 1$. Recall that the fundamental Bragg condition corresponds to $\lambda / a=2$.

There is an alternative way to look at the cut-wire pair geometry (this discussion is closely related to that in magnetic-atom design 2 in this section): For laterally well-separated cut-wire pairs, each wire of the pair basically acts as a nanoscopic $\lambda / 2$ antenna, where $\lambda$ is reduced with respect to vacuum or air due to the dielectric environment. If the two "antennas" of the cut-wire pair were identical, had identical environments, and were excited equivalently, one would obtain two effective resonances, a symmetric low-frequency mode and an antisymmetric high-frequency mode. However, the symmetry is actually already broken by the excitation geometry and by the presence of the substrate. As a result, both resulting resonances have antisymmetric character to some extent, thus, both of them have a corresponding magnetic-dipole moment connected with a resonance in the magnetic permeability $\mu$. If the wire pairs are fabricated on top of one another on a substrate as shown in Fig. 59(b), this type of "magnetic atom" is more suitable for normalincidence experiments than the split-ring resonators, because the induced magnetic-dipole moment lies in the plane of the cut-wire pair array, rather than being perpendicular to it for the SRR.

Various different parameter combinations have been systematically studied in ref. [492]. One example is the special case of $w=l$, where the cut-wire pairs turn into nanoscopic plate pairs. Their measured optical properties are shown in Fig. 60. They are closely similar to those of the SRR structures shown above. In particular, one obtains a fundamental magnetic-dipole resonance and an electric-dipole resonance at higher frequencies. The retrieval of $\epsilon(\omega)$ and $\mu(\omega)$ from the calculated spectra corresponding to the parameters of Fig. 60 yields a negative real part of $\mu(\omega)$ around $1.0 \mu \mathrm{m}$ $(1.5 \mu \mathrm{m})$ wavelength for the $w=l=200 \mathrm{~nm}(w=l=300 \mathrm{~nm})$ structure [492]. The double-plate design is amenable to fabrication on square-centimeter areas via interference lithography [495] (also see Section 2.2.3.3) or via nanoimprint lithography [496]. One group has even reported a negative real part of the refractive index around $1.5 \mu \mathrm{m}$ wavelength for cut-wire pairs [494]. Further work on cut-wire pairs has reproduced the negative real part of $\mu(\omega)$ in the near infrared [497], Ref. [498] has obtained a negative $\operatorname{Re}(\mu(\omega))$ in the visible by rotating the double-wire system by $90^{\circ}$ with respect to the substrate.

Generally, it should have become obvious from our discussion so far that any ring-like structure with one, two, or more openings in it can lead to a local magnetic-dipole moment. Fig. 61(a)-(h) summarizes the designs already discussed as well as several others.

\subsubsection{Negative-index metamaterials}

We are now well prepared to combine "magnetic atoms" and "electric atoms" in order to obtain negative-index metamaterials along the lines of Section 5.4.1. One possible design (see Fig. 62) adds metallic wires to the plate pairs discussed in magnetic-atom design 3 in this section. The wires are oriented along the electric-field vector of the incident light. These thin wires lead to a negative real part of $\epsilon(\omega)$ below the effective plasma frequency of this diluted metal. The 


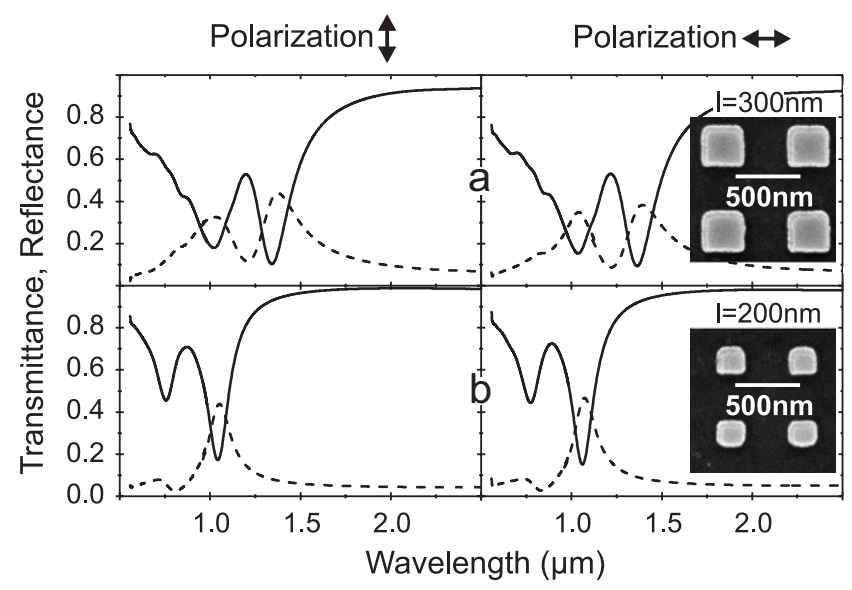

Fig. 60. Measured transmittance (solid) and reflectance (dashed) spectra for arrays of plate pairs. The incident linear polarization is indicated at the top. Parameters varied: (a) $w=l=300 \mathrm{~nm}$ and (b) $w=l=200 \mathrm{~nm}$. Fixed parameters: $t=20 \mathrm{~nm}, d=80 \mathrm{~nm}$, and $a_{x}=a_{y}=l+350 \mathrm{~nm}$. In each case, the long-wavelength transmittance dip arises from the magnetic-dipole resonance. Taken from [492].
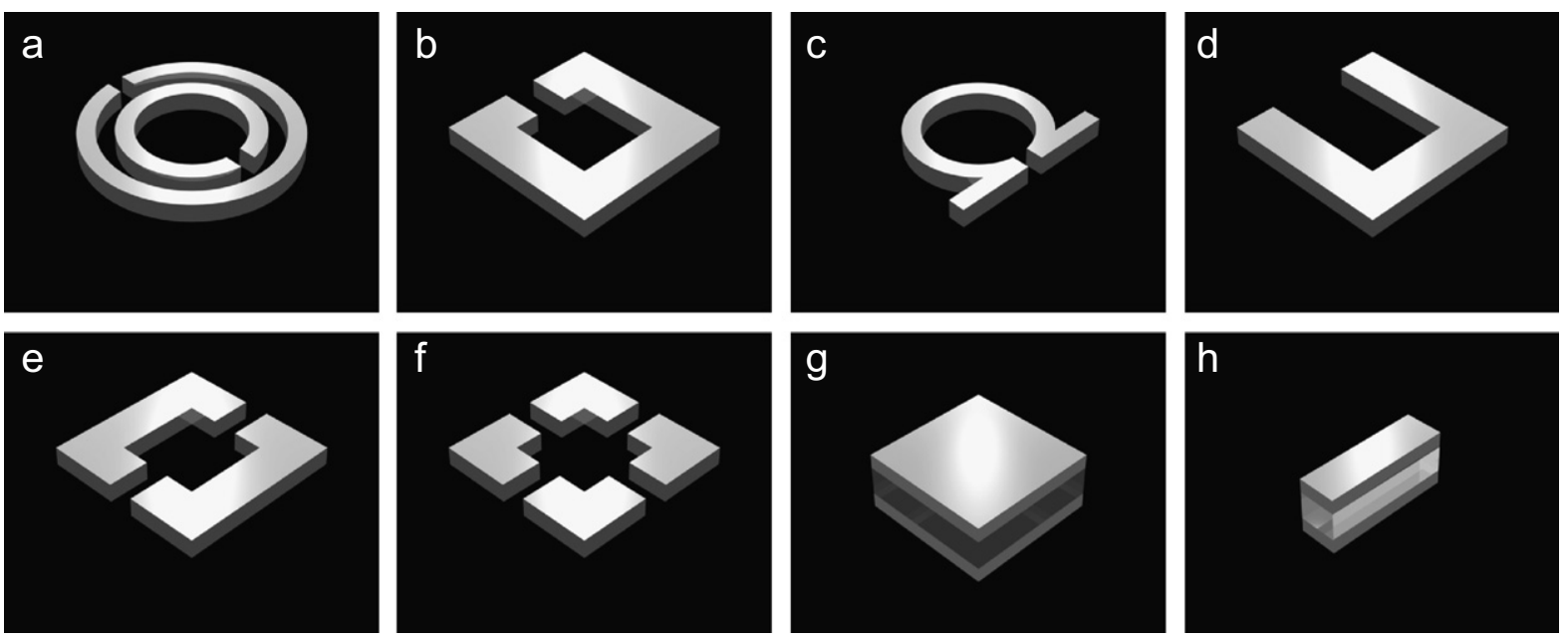

Fig. 61. (a)-(h) Illustration of a variety of different "magnetic atoms" for photonics.

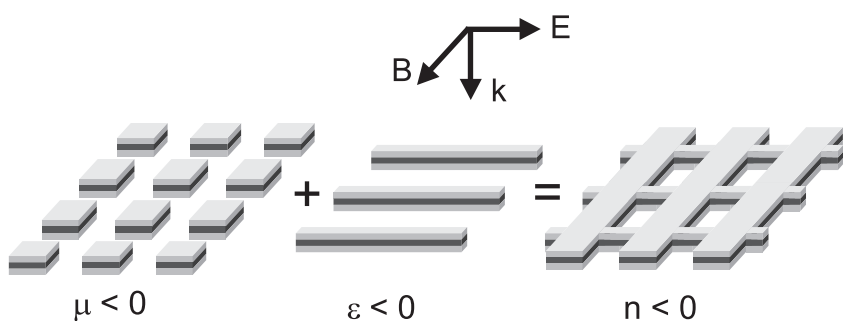

Fig. 62. Scheme of a metamaterial leading to a negative index of refraction $n$ by combining "magnetic atoms" with a negative magnetic permeability $\mu$ (cf. Fig. 60 and Figs. 61(g) and (h)) and "electric atoms" with a negative electric permittivity $\epsilon$ for the indicated polarization configuration of the incident light. Electron micrographs of fabricated structures can, e.g., be found in Refs. [499-501]. 
optimum relative width of these thin wires is a delicate compromise and depends on the desired operation wavelength (i.e., the geometry cannot simply be scaled): On the one hand, the wires should be broad to bring the effective metal plasma frequency above the desired operation frequency; on the other hand, they should be thin in order not to disturb the operation of the "magnetic atoms" of the structure. Obviously, the structure has $180^{\circ}$ rotation symmetry, leading to a pronounced polarization dependence (unlike for the quadratic plate pairs above).

The structure shown in Fig. 62 has first been proposed theoretically in Ref. [502], realized experimentally around $2 \mu \mathrm{m}$ operation wavelength in a simplified form in Ref. [503], and brought towards the visible regime as well as characterized in detail in Refs. [499-501]. Using silver (rather than, e.g., gold) as constituent metal, negative-index photonic metamaterials with the lowest losses reported so far have been realized [500]. The retrieved parameters are shown in Fig. 63. As discussed above, $\epsilon(\omega)$ corresponds to the permittivity of a diluted metal (apart from the small antiresonance on top, see Section 5.4.2), $\mu(\omega)$ to the permeability of a magnetic Lorentz oscillator. Near the $L C$

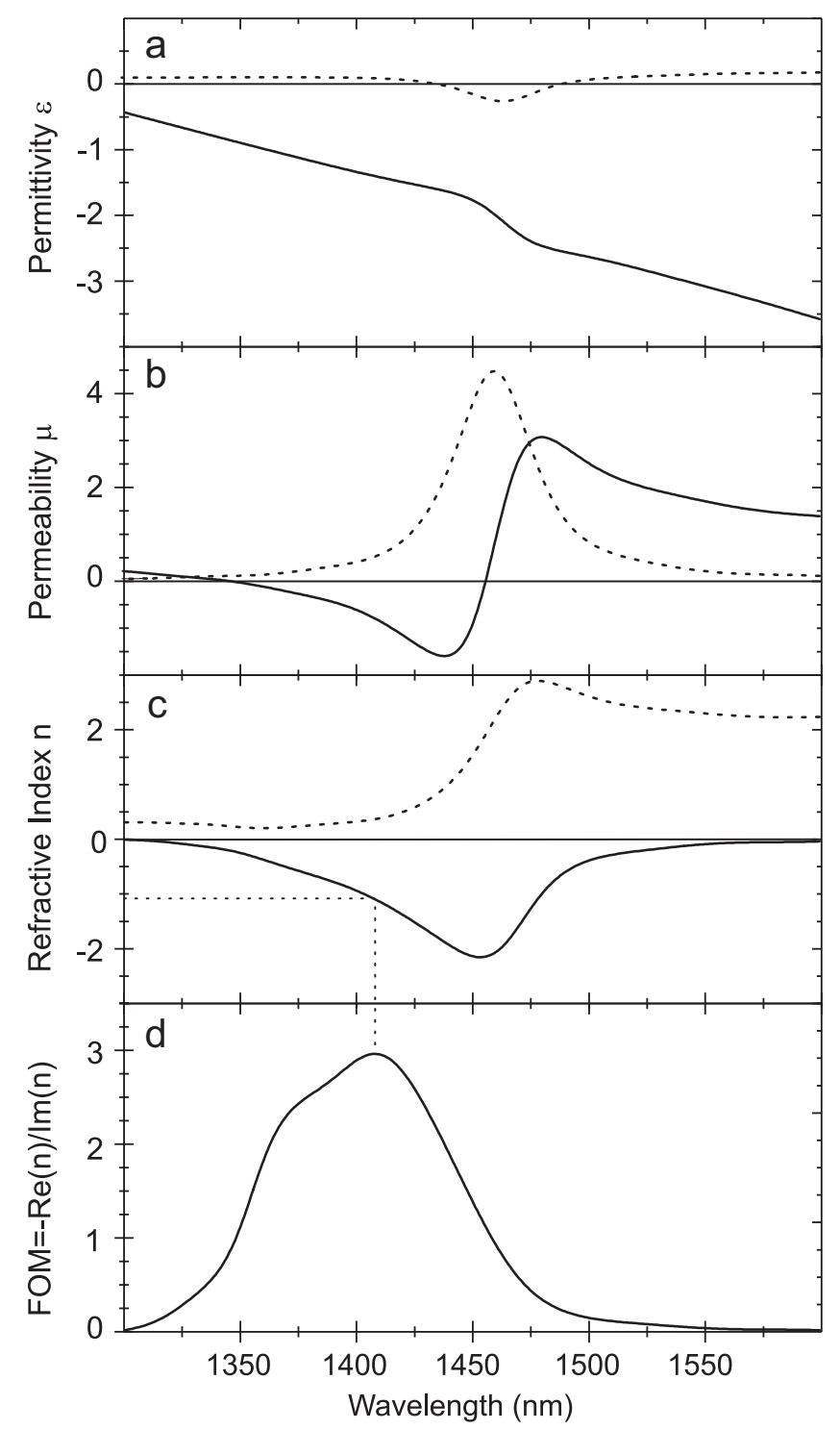

Fig. 63. (a) Retrieved permittivity $\epsilon$, (b) magnetic permeability $\mu$, and (c) the refractive index $n$ for a silver-based negative-index metamaterial design along the lines of Fig. 62. Solid curves correspond to real parts, dashed curves to imaginary parts. (d) Figure-of-merit $\operatorname{FOM}=-\operatorname{Re}(n) / \operatorname{Im}(n)($ for a lossless medium FOM $=\infty$ ). Taken from [500]. 
resonance, the real part of the refractive index becomes negative and reaches a minimum around $\operatorname{Re}(n) \approx-2$. At around $\operatorname{Re}(n) \approx-1$, the negative real part of $n$ is three times larger than its imaginary part. Miniaturizing and readjusting the metamaterial parameters, silver as constituent metal also allows for achieving a negative index of $\operatorname{Re}(n) \approx-0.6$ at around $780 \mathrm{~nm}$ wavelength, i.e., at the red end of the visible regime [501].

In "Example 3" in Section 5.4.1, we have shown that a negative real part of the refractive index $n(\omega)$ means that the phase velocity vector $\vec{v}_{\text {phase }}$ (or the wave vector $\vec{k}$ ) of light and the Poynting vector $\vec{S}$ are antiparallel, i.e., $\vec{v}_{\text {phase }} \cdot \vec{S}<0$ or equivalently $\vec{k} \cdot \vec{S}<0$ for the frequency $\omega$. Sometimes, it is assumed that $\vec{S} \cdot \vec{v}_{\text {group }}>0$ holds for the group velocity vector of light $\vec{v}_{\text {group }}$. This would lead to $\vec{v}_{\text {phase }} \cdot \vec{v}_{\text {group }}<0$ for a negative-index metamaterial. Note that this formulation is indeed correct for the fictitious case of strictly vanishing losses - a case, however, which does not even exist conceptually for negative-index metamaterials. Mathematically [499], the group velocity is generally given by (in one-dimensional form)

$$
v_{\text {group }}(\omega)=\frac{\mathrm{d} \omega}{\mathrm{d} k}=\frac{c_{0}}{\operatorname{Re}(n(\omega))+\omega \frac{\mathrm{dRe}(n(\omega))}{\mathrm{d} \omega}} .
$$

For the purely mathematical case of $\operatorname{Re}(n(\omega))=$ const. $<0$ for all frequencies, the group velocity would obviously be identical to the phase velocity of light. Negative phase velocity would result in negative group velocity as well. We have discussed above, however, that a negative-index metamaterial is inherently and unavoidably dispersive, i.e., $\mathrm{d} \operatorname{Re}(n(\omega)) / \mathrm{d} \omega \neq 0$. For $\operatorname{Re}(n(\omega))<0$, the group velocity becomes positive if and only if the denominator on the RHS of (5.166) is positive.

Phase and group velocity of light in a negative-index metamaterial can directly be measured via interferometric "time-of-flight" experiments [499]. Consider the propagation of a Gaussian electromagnetic wave packet (a pulse) over a distance $d>0$ through some material. For constant velocity $v$, the time-of-flight $t$ is simply given by $t=d / v$. Regarding the phase of the wave, we obtain the phase propagation time $t_{\text {phase }}=d / v_{\text {phase }}$. With respect to the envelope of the pulse (the "group"), we obtain the group propagation time $t_{\text {group }}=d / v_{\text {group }}$. Note that the propagation times $t_{\text {phase }}$ and $t_{\text {group }}$, respectively, become negative if $v_{\text {phase }}$ and $v_{\text {group }}$, respectively, are negative. These times can be measured by introducing the metamaterial into one arm of a Michelson interferometer. In essence, one records two interferograms, one with the metamaterial sample on its glass substrate in one of the interferometer arms, and a second interferogram with just the glass substrate by laterally moving the metamaterial out of the optical path. The corresponding shift on the interferometer time delay axis, $\Delta t_{\text {phase }}=t_{\text {phase }}$ (meta) $-t_{\text {phase }}$ (vac), yields the phase delay due to the metamaterial. By tuning the center wavelength of the incident Gaussian pulses and by repeating the described procedure for each wavelength, one can measure phase-delay spectra. Simultaneously and similarly, one can infer the shift $\Delta t_{\text {group }}=t_{\text {group }}($ meta $)-t_{\text {group }}(\mathrm{vac})$ between the two Gaussian interferogram envelopes at each center wavelength, which provides the group-delay spectra. Corresponding data are shown in Fig. 64 for a silver-based structure described in [500]. The analysis reveals that the phase velocity and the group velocity are negative simultaneously in a certain spectral region [499]. Subtleties arise from phase factors due to the sample interfaces [499].

While these experiments on negative-index photonic metamaterials are a convincing proof-of-principle [504,505], there is plenty of room left for future improvements: First, the losses need to be reduced equivalent to increasing the figure-of-merit (FOM) as introduced in the caption of Fig. 63. Possibly, losses can be compensated by introducing gain materials or by using parametric optical amplification [506]. Second, truly three-dimensional photonic metamaterials (rather than just single two-dimensional layers) need to be designed and fabricated. First theoretical [507] and experimental [508] steps have been described. Third, isotropic three-dimensional negative-index metamaterials are desirable. Clearly, the designs discussed in this subsection are highly nonisotropic and, moreover, exhibit a pronounced polarization dependence. Indeed, with regard to theory, the retrieval of effective isotropic functions $\epsilon(\omega)$ and $\mu(\omega)$ for structures that are obviously not isotropic at all, is not fully satisfactory. In general, $\epsilon$ and $\mu$ have to be replaced by tensorial quantities. At this point, it is useful to recall that the restriction to a magnetic-permeability tensor (and/or to an electric-permittivity tensor) alone already implies that the magnetic-dipole moments are exclusively induced by the magnetic-field component of the light (and, similarly, that the electric-dipole moments are exclusively induced by the electric-field component of the light). In contrast to this, the discussion of experiments on SRR above has explicitly shown that, for example, the electric-field component of the light can also induce a magnetic-dipole moment normal to the SRR plane. Thus, such "cross-terms" have to be accounted for as well. Materials of this sort are referred to as bi-anisotropic [509]. 


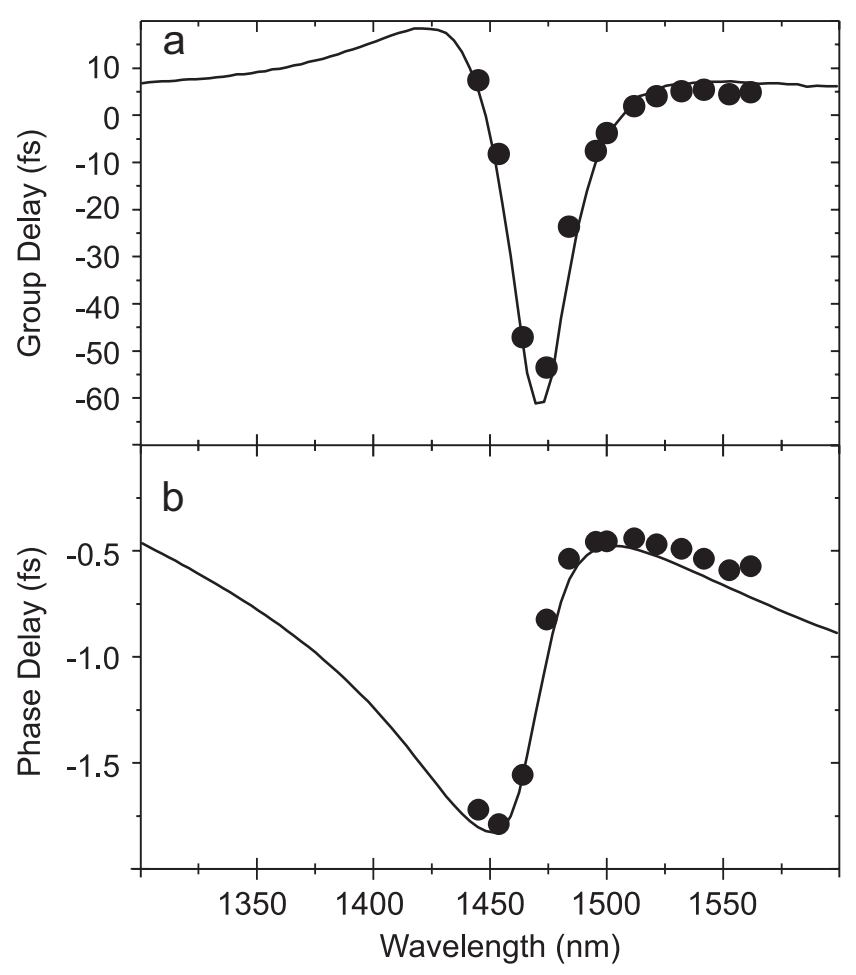

Fig. 64. Interferometric "time-of-flight" experiments (dots) on the identical silver-based negative-index metamaterial [500] as in Figs. 62 and 63. The solid curves are numerical calculations with parameters identical to those in Fig. 63. (a) group-delay $\Delta t_{\text {group }}$ spectrum, (b) phase-delay $\Delta t_{\text {phase }}$ spectrum. Reprinted figure with permission of G. Dolling. Similar data on a gold-based structure have been published in Ref. [499].

Finally, we briefly mention that an alternative route towards metamaterials with a negative phase velocity of light has been described theoretically in Ref. [510]. This approach takes advantage of the avoided crossing arising from a chiral medium with an electronic resonance and leads to a negative phase velocity for one of the two circular polarizations. Other work on planar chiral periodic metallic structures (however, not all metamaterials in the sense used here, i.e., no effective materials) is discussed in Refs. [511-516]. Broadly speaking, the aspect of chirality in photonic metamaterials has not fully been explored yet.

\section{Conclusions and outlook}

The concepts of photonic crystals and photonic metamaterials have changed the spirit of optics and photonics. Researchers no longer just study and use the rich variety of optical materials provided by nature but have rather become creative designers who tailor optical properties almost at will, often leading to unexpected and unprecedented behavior. Examples are three-dimensional photonic band gaps, giant circular dichroism without static magnetic fields, magnetism at optical frequencies, or a negative index of refraction.

In natural optical crystals, the wavelength of light $\lambda$ is about three orders of magnitude larger than the lattice constant $a$, i.e., $\lambda \approx 1000 a$. For metamaterials one ideally has $\lambda \gg a$, for photonic-crystals the condition $\lambda \approx a$ holds. The optical properties of metamaterials are mainly determined by the properties of the tailored unit cell. Thus, metamaterials can be described by an effective electric permittivity $\epsilon$ and an effective magnetic permeability $\mu$. For photonic crystals, the interaction between the different unit cells is of crucial importance. This strong interaction or coupling leads to Bragg scattering, Bloch waves, and a photonic band structure that can exhibit a complete photonic band gap under appropriate conditions.

We have reviewed corresponding experiments and theory in one, two and three dimensions, addressing linear-optical, nonlinear-optical, and quantum-optical aspects. Most of the work focusses on dielectric photonic crystals and metallic metamaterials. However, metallic photonic crystals and dielectric metamaterials have also been covered. 
Future experimental challenges mainly lie in the area of three-dimensional periodic nanostructures for photonics. Regarding fabrication of three-dimensional photonic crystals and photonic-band-gap materials, progress has been dramatic throughout the last years. As a result, nonlinear-optical and quantum-optical experiments as well as applications come into reach. In contrast, truly three-dimensional photonic metamaterials have not been realized at all to date. So far, at most three functional layers have been studied experimentally.

With respect to theory, the field is still open for surprises and new effects. Intuitive guidelines based on symmetry and topology considerations addressing the question, which photonic-crystal structure leads to significant photonic band gaps, still remain elusive. Furthermore, especially regarding quantum-optical aspects, theory has not yet reached fully (quantitative) predictive character in many cases. Thus, novel analytical as well as numerical techniques and approaches have to be developed, and existing ones have to be improved. Such tools would also be valuable for optimizing existing designs for photonic crystals and metamaterials.

The vision of the reviewed field is to design and fabricate artificial materials that allow for realizing unique novel electromagnetic properties that cannot be achieved with natural materials. In this enterprise, only our own imagination and creativity set the future limits.

\section{Acknowledgments}

We are indebted to the many undergraduate and graduate students in our Karlsruhe research groups for their dedication and hard work. Furthermore, we thank S. John, G. A. Ozin, C. M. Soukoulis, and R. B. Wehrspohn for many stimulating discussions. This review has (finally) been completed by two of us (K.B. and M.W.) at a "Sommerakademie" of the Studienstiftung des Deutschen Volkes in Guidel (France) 2006. We acknowledge support by the Center for Functional Nanostructures (CFN) of the Deutsche Forschungsgemeinschaft (DFG) within subprojects A1.1 to A1.5. The research of K.B and L.T. is or was further supported by the DFG-Priority Program SPP 1113 "Photonic Crystals" under Grant Bu-1107/3-1, 3-2, and 6-1, that of G.v.F. by project DFG-Fr 1671/4-3, that of S.L. through a "Helmholtz-HochschulNachwuchsgruppe" (VH-NG-232), and that of M.W. by project DFG-We 1497/9-1.

\section{References}

[1] L. Brillouin, Wave Propagation in Periodic Structures, McGraw-Hill, New York, 1946.

[2] L. Brillouin, Wave Propagation and Group Velocity, Academic Press, New York, 1960.

[3] P. Yeh, J. Opt. Soc. Am. 69 (1979) 742.

[4] J. Peatross, S.A. Glasgow, M. Ware, Average energy flow of optical pulses in dispersive media, Phys. Rev. Lett. 84 (2000) 2370.

[5] E. Yablonovitch, Phys. Rev. Lett. 58 (1987) 2059.

[6] S. John, Phys. Rev. Lett. 58 (1987) 2486.

[7] K.-M. Ho, C.T. Chan, C.M. Soukoulis, Phys. Rev. Lett. 65 (1990) 3152.

[8] N.W. Ashcroft, N.D. Mermin, Solid State Physics, Harcourt Brace Jovanovich College Publishers, 1976.

[9] K. Sakoda, Optical Properties of Photonic Crystals, Series in Optical Sciences, vol. 80, Springer, Berlin, 2001.

[10] J. Harrison, P. Kuchment, A. Sobolev, B. Winn, math-ph/0702035, 2007.

[11] A. Kirchner, K. Busch, C.M. Soukoulis, Phys. Rev. B 57 (1998) 277.

[12] S. Datta, C.T. Chan, K.M. Ho, C.M. Soukoulis, Phys. Rev. B 48 (1993) 014936.

[13] K. Inoue, K. Ohtaka (Eds.), Photonic Crystals: Physics, Fabrication, and Applications, Series in Optical Sciences, vol. 94, Springer, Berlin, 2004.

[14] K. Busch, S. John, Phys. Rev. Lett. 83 (1999) 967.

[15] K. Yoshino, Y. Shimoda, Y. Kawagishi, K. Nakayama, M. Ozaki, Appl. Phys. Lett. 75 (1999) 932.

[16] H. Takeda, K. Yoshino, Phys. Rev. E 67 (2003) 056607.

[17] H. Takeda, K. Yoshino, Phys. Rev. B 67 (2003) 073106.

[18] S.F. Mingaleev, M. Schillinger, D. Hermann, K. Busch, Opt. Lett. 29 (2004) 2858.

[19] E.P. Kosmidou, E.E. Kriezis, T.D. Tsiboukis, IEEE J. Quant. Electron. 41 (2005) 657.

[20] Z. Wang, S. Fan, Appl. Phys. B 81 (2005) 369.

[21] Z. Wang, S. Fan, Opt. Lett. 30 (2005) 1989.

[22] A. Hache, L. Tkeshelashvili, M. Diem, K. Busch, Europhys. Lett. 83 (2006) 967.

[23] A. Hache, M. Malik, M. Diem, L. Tkeshelashvili, K. Busch, Photonics Nanostruct. 5 (2007) 29.

[24] S.W. Leonard, J.P. Mondia, H.M. van Driel, O. Toader, S. John, K. Busch, A. Birner, U. Gösele, V. Lehmann, Phys. Rev. B 61 (2000) R2389.

[25] G. Mertens, T. Röder, P. Schweins, K. Huber, H.S. Kitzerow, Appl. Phys. Lett. 80 (2002) 1885.

[26] G. Mertens, T. Röder, H. Matthias, H. Marsmann, H.S.R. Kitzerow, S.L. Schweizer, C. Jamois, R.B. Wehrspohn, M. Neubert, Appl. Phys. Lett. 83 (2003) 3036.

[27] Ch. Schuller, F. Klopf, J.P. Reithmaier, M. Kamp, A. Forchel, Appl. Phys. Lett. 82 (2003) 2767. 
[28] H. Matthias, T. Röder, R.B. Wehrspohn, H.S. Kitzerow, S. Matthias, S.J. Picken, Appl. Phys. Lett. 87 (2005) 241105.

[29] R. van der Heijden, C.F. Carlström, J.A.P. Snijders, R.W. van der Heijden, F. Karouta, R. Nötzel, H.W.M. Salemink, Appl. Phys. Lett. 88 (2006) 161112.

[30] J. Martz, R. Ferrini, F. Nüesch, L. Zuppiroli, B. Wild, L.A. Dunbar, R. Houdre, M. Mulot, S. Anand, J. Appl. Phys. 99 (2006) 103105.

[31] R. Sprik, B.A. van Tiggelen, A. Lagendijk, Europhys. Lett. 35 (1996) 265.

[32] K. Busch, S. John, Phys. Rev. E 58 (1998) 3896.

[33] G. Lehmann, M. Taut, Phys. Stat. Sol. (b) 54 (1972) 469.

[34] J. Hanna, M. Watanabe, T. Kato, J. Phys.: Condens. Matter 2 (1990) 7445.

[35] R. Wang, X.-H. Wang, B.-Y. Gu, G.-Z. Yang, Phys. Rev. B 67 (2003) 155114.

[36] J. Zarbakhsh, F. Hagmann, S.F. Mingaleev, K. Busch, K. Hingerl, Appl. Phys. Lett. 84 (2004) 4687.

[37] Y.S. Chan, C.T. Chan, Z.Y. Liu, Phys. Rev. Lett. 80 (1998) 965.

[38] A.A. Asatryan, K. Busch, R.C. McPhedran, L.C. Botton, C.M. de Sterke, N.A. Nicorovici, Waves Random Media 13 (2003) 9.

[39] K. Busch, S. John, Phys. Rev. E 58 (1998) 3896.

[40] M. Lax, Symmetry Principles in Solid State and Molecular Physics, Dover Publications, 1974.

[41] C.M. de Sterke, J.E. Sipe, Phys. Rev. A 38 (1988) 5149.

[42] D. Hermann, M. Frank, K. Busch, P. Wölfle, Opt. Expr. 8 (2001) 167.

[43] J.E. Sipe, Phys. Rev. E 62 (2000) 5672.

[44] P.T. Rakich, M.S. Dahlem, S. Tandon, M. Ibanescu, M. Soljacic, G.S. Petrich, J.D. Joannopoulos, L.A. Kolodziejski, E.P. Ippen, Nat. Mater. 5 (2006) 93.

[45] X. Yu, S. Fan, Appl. Phys. Lett. 83 (2003) 3251.

[46] S. Foteinopoulou, E.N. Economou, C.M. Soukoulis, Phys. Rev. Lett. 90 (2003) 107402.

[47] S. Foteinopoulou, C.M. Soukoulis, Phys. Rev. B 72 (2005) 165112.

[48] M. Born, E. Wolf, Principles of Optics, Pergamon Press, London, 1959.

[49] V.G. Veselago, Sov. Phys. Usp. 10 (1968) 509.

[50] P.St.J. Russell, Phys. Rev. A 33 (1986) 3232.

[51] R. Zengerle, J. Mod. Opt. 34 (1987) 1589.

[52] H. Kosaka, T. Kawashima, A. Tomita, M. Notomi, T. Tamamura, T. Sato, S. Kawakami, Phys. Rev. B 58 (1998) R10096.

[53] H. Kosaka, T. Kawashima, A. Tomita, M. Notomi, T. Tamamura, T. Sato, S. Kawakami, Appl. Phys. Lett. 74 (1999) 1212.

[54] H. Kosaka, T. Kawashima, A. Tomita, M. Notomi, T. Tamamura, T. Sato, S. Kawakami, Appl. Phys. Lett. 74 (1999) 1370.

[55] W. Hergert, M. Däne, Phys. Stat. Sol. (a) 197 (2003) 620.

[56] W.M. Robertson, J. Arjavalingam, R.D. Meade, K.D. Brommer, A.M. Rappe, J.D. Joannopoulos, Phys. Rev. Lett. 68 (1992) 2023.

[57] W.M. Robertson, J. Arjavalingam, R.D. Meade, K.D. Brommer, A.M. Rappe, J.D. Joannopoulos, J. Opt. Soc. Am. B 10 (1993) 322.

[58] K. Sakoda, Phys. Rev. B 52 (1995) 7982.

[59] K. Sakoda, Phys. Rev. B 51 (1995) 4672.

[60] K.W.-K. Shung, Y.C. Tsai, Phys. Rev. B 48 (1993) 11265.

[61] A.V. Zayats, I.I. Smolyaninov, A.A. Maradudin, Phys. Rep. 408 (2005) 131.

[62] E. Istrate, E.H. Sargent, Rev. Mod. Phys. 78 (2006) 466.

[63] Yu.A. Vlasov, N. Moll, S.J. McNab, Opt. Lett. 29 (2004) 2175.

[64] P. Kramper, M. Agio, C.M. Soukoulis, A. Birner, F. Müller, R.B. Wehrspohn, U. Gösele, V. Sandoghdar, Phys. Rev. Lett. 92 (2004) 113903.

[65] R.D. Meade, K.D. Brommer, A.M. Rappe, J.D. Joannopoulos, Phys. Rev. B 44 (1991) 10961.

[66] J.M. Elson, P. Tran, Phys. Rev. B 54 (1996) 1711.

[67] W.M. Robertson, J. Arjavalingam, R.D. Meade, K.D. Brommer, A.M. Rappe, J.D. Joannopoulos, Opt. Lett. 18 (1993) 528.

[68] A. Mekis, J.C. Chen, I. Kurland, S. Fan, P.R. Villeneuve, J.D. Joannopoulos, Phys. Rev. Lett. 77 (1996) 3787.

[69] S. Fan, P.R. Villeneuve, J.D. Joannopoulos, H.A. Haus, Phys. Rev. Lett. 80 (1998) 960.

[70] K. Busch, S. Lölkes, R.B. Wehrspohn, H. Föll (Eds.), Photonic Crystals: Advances in Design, Fabrication, and Characterization, Wiley-VCH, 2004.

[71] S. Satpathy, Z. Zhang, M.R. Salehpour, Phys. Rev. Lett. 65 (1990) 2478.

[72] K.M. Leung, Y.F. Liu, Phys. Rev. Lett. 65 (1990) 2646.

[73] P.R. Villeneuve, M. Piche, Prog. Quant. Electron. 18 (1994) 153.

[74] R.D. Meade, A.M. Rappe, K.D. Brommer, J.D. Joannopoulos, Phys. Rev. B 48 (1993 Erratum: Phys. Rev. B 55 (1997) 15942$) 8434$.

[75] S. Johnson, J.D. Joannopoulos, Opt. Expr. 8 (2001) 173.

[76] G.J. Pearce, T.D. Hedley, D.M. Bird, Phys. Rev. B 71 (2005) 195108.

[77] E. Istrate, A.A. Green, E.H. Sargent, Phys. Rev. B 71 (2005) 195122.

[78] L. Li, J. Opt. Soc. Am. A 13 (1996) 1024.

[79] L. Li, J. Opt. Soc. Am. A 13 (1996) 1870.

[80] M.G. Moharam, E.B. Grann, D.A. Pommet, T.K. Gaylord, J. Opt. Soc. Am. A 12 (1995) 1068.

[81] G. Granet, B. Guizal, J. Opt. Soc. Am. A 13 (1996) 1019.

[82] P. Lalanne, G.M. Morris, J. Opt. Soc. Am. A 13 (1996) 779.

[83] L. Li, J. Opt. Soc. Am. A 14 (1997) 2758.

[84] D.M. Whittaker, I.S. Culshaw, Phys. Rev. B 60 (1999) 2610.

[85] S.G. Tikhodeev, A.L. Yablonskii, E.A. Muljarov, N.A. Gippius, T. Ishihara, Phys. Rev. B 66 (2002) 045102.

[86] G. von Freymann, W. Koch, D.C. Meisel, M. Wegener, M. Diem, A. Garcia-Martin, S. Pereira, K. Busch, J. Schilling, R.B. Wehrspohn, U. Gösele, Appl. Phys. Lett. 83 (2003) 614. 
[87] M. Deubel, G. von Freymann, M. Wegener, S. Pereira, K. Busch, C.M. Soukoulis, Nat. Mater. 3 (2004) 444.

[88] M. Deubel, M. Wegener, S. Linden, G. von Freymann, Appl. Phys. Lett. 87 (2005) 221104.

[89] V.N. Astratov, D.M. Whittaker, I.S. Culshaw, R.M. Stevenson, M.S. Skolnick, T.F. Krauss, R.M. De La Rue, Phys. Rev. B 60 (1999) R16255.

[90] D.C. Meisel, M. Diem, M. Deubel, F. Pérez-Willard, S. Linden, D. Gerthsen, K. Busch, M. Wegener, Adv. Mater. 18 (2006) 2964.

[91] P. Lalanne, E. Silberstein, Opt. Lett. 15 (2000) 1092.

[92] J.P. Hugonin, P. Lalanne, J. Opt. Soc. Am. A 22 (2006) 1844.

[93] K.S. Yee, IEEE Trans. Antennas Propagat. 14 (1966) 302.

[94] A. Taflove, S.C. Hagness, Computational Electrodynamics: The Finite-Difference Time-Domain Method, third ed., Artech House, Boston, MA, 2005.

[95] J.P. Berenger, J. Comp. Phys. 114 (1994) 185.

[96] M. Koshiba, Y. Tsuji, S. Sasaki, IEEE Microwave Wireless Components Lett. 11 (2001) 152.

[97] A. Mohammadi, H. Nadgaran, M. Agio, Opt. Expr. 13 (2005) 10367.

[98] A. Ditkowski, K. Dridi, J.S. Hesthaven, J. Comp. Phys. 170 (2001) 39.

[99] M. Fuji, W.J.R. Hoefer, IEEE Trans. Microwave Theory Tech. 46 (1998) 2463.

[100] T. Namiki, IEEE Trans. Microwave Theory Techn. 48 (2000) 1743.

[101] K.L. Shlager, J.B. Schneider, IEEE Trans. Antennas Propagat. 51 (2003) 642.

[102] J. Jin, The Finite Element Method in Electromagnetics, second ed., Wiley, New York, 2002.

[103] J.S. Kole, M.T. Figge, H. De Raedt, Phys. Rev. E 64 (2001) 66705.

[104] H. De Raedt, K.F.L. Michielsen, J.S. Kole, M.T. Figge, IEEE Trans. Antennas Propagat. 51 (2003) 3155.

[105] A.G. Borisov, S.V. Shabanov, J. Comp. Phys. 216 (2006) 391.

[106] J. Niegemann, L. Tkeshelashvili, K. Busch, J. Comput. Theor. Nanosci. 4 (2007) 627.

[107] S. Burger, R. Klose, A. Schädle, F. Schmidt, L. Zschiedrich, Proc. SPIE 5728 (2005) 164.

[108] P.I. Borel, A. Harpøth, L.H. Frandsen, M. Kristensen, S. Peixiong, J.S. Jensen, O. Sigmund, Opt. Expr. 12 (2004) 1996.

[109] A. Tetu, K. Kristensen, L.H. Frandsen, A. Harpøth, P.I. Borel, J.S. Jensen, O. Sigmund, Opt. Expr. 13 (2005) 8606.

[110] L.E. Peterson, J. Jin, IEEE Trans. Antennas Propagat. 54 (2006) 12.

[111] S. Wang, R. Lee, F.L. Texeira, IEEE Trans. Antennas Propagat. 54 (2006) 20.

[112] C. Hafner, Post-Modern Electromagnetics: Using Intelligent MaXwell Solvers, Wiley, New York, 1999.

[113] K.M. Leung, Y. Qiu, Phys. Rev. B 48 (1993) 7767.

[114] N.A. Nicorovici, R.C. McPhedran, L.C. Botten, Phys. Rev. E 52 (1995) 1135.

[115] K. Ohtaka, Phys. Rev. B 19 (1979) 5067.

[116] X. Wang, Z.G. Zhang, Q. Yiu, B.N. Harmon, Phys. Rev. B 47 (1993) 4161.

[117] A. Moroz, J. Phys.: Condens. Matter 6 (1994) 171.

[118] J. Smajic, C. Hafner, D. Erni, Opt. Expr. 11 (2003) 566.

[119] J. Smajic, C. Hafner, D. Erni, Opt. Expr. 11 (2003) 1378.

[120] N. Stefanou, V. Karathanos, A. Modinos, J. Phys.: Condens. Matter 4 (1992) 7389.

[121] L.-M. Li, Z.-Q. Zhang, Phys. Rev. B 58 (1998) 9587.

[122] N. Stefanou, A. Modinos, J. Phys. Condens. Matter 5 (1993) 8859.

[123] A.A. Asatryan, P.A. Robinson, L.C. Botten, R.C. McPhedran, N.A. Nicorovici, C.M. de Sterke, Phys. Rev. E 52 (1995) 1135.

[124] G.H. Wannier, Phys. Rev. 52 (1937) 191.

[125] W. Kohn, Phys. Rev. 115 (1959) 809.

[126] K. Busch, S.F. Mingaleev, A. Garcia-Martin, M. Schillinger, D. Hermann, J. Phys.: Condens. Matter 15 (2003) R1233.

[127] N. Marzari, D. Vanderbilt, Phys. Rev. B 56 (1997) 12847.

[128] I. Souza, N. Marzari, D. Vanderbilt, Phys. Rev. B 65 (2002) 035109.

[129] A. Garcia-Martin, D. Hermann, K. Busch, P. Wölfle, Mater. Res. Soc. Symp. Proc. 722 (2002) L1.1.

[130] A. Garcia-Martin, D. Hermann, F. Hagmann, K. Busch, P. Wölfle, Nanotechnology 14 (2003) 177.

[131] D.M. Whittaker, M.P. Croucher, Phys. Rev. B 67 (2003) 085204.

[132] S.F. Mingaleev, K. Busch, Opt. Lett. 28 (2003) 619.

[133] Y. Jiao, S. Fan, D.A.B. Miller, Opt. Lett. 30 (2005) 141.

[134] Y. Jiao, S. Fan, D.A.B. Miller, Opt. Lett. 30 (2005) 302.

[135] Y. Jiao, S.F. Mingaleev, M. Schillinger, D.A.B. Miller, S. Fan, K. Busch, IEEE Phot. Tech. Lett. 17 (2005) 1875.

[136] K.M. Leung, J. Opt. Soc. Am. B 10 (1993) 303.

[137] J.P. Albert, C. Jouanin, D. Cassagne, D. Bertho, Phys. Rev. B 61 (2000) 4381.

[138] E. Lidorikis, M.M. Sigalas, E.N. Economou, C.M. Soukoulis, Phys. Rev. Lett. 81 (1998) 1405.

[139] O. Painter, K. Srinivasan, P.E. Barclay, Phys. Rev. B 68 (2003) 035214.

[140] S. Longhi, Phys. Rev. E 71 (2005) 016603.

[141] E.N. Economou, Green Functions in Quantum Physics, Springer, Berlin, 1983.

[142] J.D. Jackson, Classical Electrodynamics, third ed., Wiley, New York, 1999.

[143] P.M. Morse, H. Feshbach, Methods of Theoretical Physics, McGraw-Hill, New York, 1953.

[144] S.F. Mingaleev, Y.S. Kivshar, Phys. Rev. Lett. 86 (2001) 5474.

[145] S.F. Mingaleev, Y.S. Kivshar, J. Opt. Soc. Am. B 19 (2002) 2241.

[146] A.R. McGurn, J. Phys. D 28 (2005) 2338.

[147] S. Hughes, L. Ramunno, J.F. Young, J.E. Sipe, Phys. Rev. Lett. 94 (2005) 033903. 
[148] A.R. Cowan, J.F. Young, Phys. Rev. B 65 (2002) 085106.

[149] A.R. Cowan, J.F. Young, Phys. Rev. E 68 (2003) 046606.

[150] N. Matuschek, F.X. Kärtner, U. Keller, IEEE J. Selected Topics Quantum Electron. 4 (1998) 197.

[151] N. Matuschek, F.X. Kärtner, U. Keller, IEEE J. Quantum Electron. 5 (1999) 129.

[152] J.N. Winn, Y. Fink, S. Fan, J.D. Joannopoulos, Opt. Lett. 23 (1998) 1573.

[153] Y. Fink, J.N. Winn, S. Fan, C. Chen, J. Michel, J.D. Joannopoulos, E.L. Thomas, Science 282 (1998) 1679.

[154] C. Hooijer, D. Lenstra, A. Lagendijk, Opt. Lett. 25 (2000) 1666.

[155] R. Sapienza, P. Costantino, D. Wiersma, M. Ghulinyan, C.J. Oton, L. Pavesi, Phys. Rev. Lett. 91 (2003) 263902.

[156] M. Hübner, J. Kuhl, T. Strouken, A. Knorr, S.W. Koch, R. Hey, K. Ploog, Phys. Rev. Lett. 76 (1996) 4199.

[157] G.P. Agrawal, Nonlinear Fiber Optics, Academic Press, London, 2001.

[158] M. Bayer, T. Gutbrod, A. Forchel, T.L. Reinecke, P.A. Knipp, R. Werner, J.P. Reithmaier, Phys. Rev. Lett. 83 (1999) 5374.

[159] A. Yariv, Y. Xu, R.K. Lee, A. Scherer, Opt. Lett. 24 (1999) 711.

[160] A.L. Jones, J. Opt. Soc. Am. 55 (1965) 261.

[161] S. Somekh, E. Garmire, A. Yariv, H.L. Garvin, R.G. Hunsberger, Appl. Phys. Lett. 22 (1973) 46.

[162] G.I. Stegeman, M. Segev, Science 286 (1999) 1518.

[163] D.M. Christodoulidis, F. Lederer, Y. Silberberg, Nature 424 (2003) 817.

[164] see special issue: Opt. Expr. 13 (2005).

[165] H. Eisenberg, Y. Silberberg, R. Morandotti, J.S. Aitchison, Phys. Rev. Lett. 85 (2000) 1863.

[166] T. Pertsch, T. Zentgraf, U. Peschel, A. Brauer, F. Lederer, Phys. Rev. Lett. 88 (2002) 93901.

[167] T. Pertsch, P. Dannberg, W. Elflein, A. Brauer, F. Lederer, Phys. Rev. Lett. 83 (1999) 4752.

[168] R. Morandotti, U. Peschel, J.S. Eisenberg, Y. Silberberg, Phys. Rev. Lett. 83 (1999) 4756.

[169] H. Trompeter, T. Pertsch, F. Lederer, D. Michaelis, U. Streppel, A. Bräuer, U. Peschel, Phys. Rev. Lett. 96 (2006) 023901.

[170] R. Iwanow, D.A. May-Arrioja, D.M. Christodoulidis, G.I. Stegeman, Y. Min, W. Sohler, Phys. Rev. Lett. 95 (2005) 53902.

[171] F. Keller, M.S. Hunter, D.L. Robinson, J. Electrochem. Soc. 100 (1953) 411.

[172] A. Uhlir, Bell System Tech. J. 35 (1956) 333.

[173] H. Masuda, K. Fukuda, Science 268 (1994) 1466.

[174] V. Lehmann, H. Föll, J. Electrochem. Soc. 137 (1990) 653.

[175] V. Lehmann, J. Electrochem. Soc. 140 (1993) 2836.

[176] X.G. Zhang, Electrochemistry of Silicon and its Oxides, Kluwer Academic, Plenum Publisher, New York, 2001.

[177] V. Lehmann, Electrochemistry of Silicon, Wiley-VCH, Weinheim, 2002.

[178] M. Föll, M. Christopherson, J. Carstensen, G. Hasse, Mater. Sci. Eng. R 180 (2002) 1.

[179] R.B. Wehrspohn, J. Schilling, J. Choi, Y. Luo, S. Matthias, S.L. Schweizer, F. Müller, U. Gösele, S. Lölkes, S. Langa, J. Carstensen, H. Föll, Photonic Crystals, Wiley-VCH, Weinheim, 2004 p. 63.

[180] A. Birner, R.B. Wehrspohn, U.M. Gösele, K. Busch, Adv. Mater. 13 (2001) 377.

[181] M. Augustin, G. Böttger, M. Eich, C. Etrich, H.-J. Fuchs, R. Iliew, U. Hübner, M. Kessler, E.-B. Kley, F. Lederer, C. Liguda, S. Nolte, H.G. Meyer, W. Morgenroth, U. Peschel, A. Petrov, D. Schelle, M. Schmidt, A. Tünnermann, W. Wischmann, Photonic Crystals, Wiley-VCH, Weinheim, 2004 p. 289.

[182] S.-I. Takayama, H. Kitagawa, Y. Tanaka, T. Asano, S. Noda, Appl. Phys. Lett. 87 (2005) 61107.

[183] L.J. Wu, M. Mazilu, T. Karle, T.F. Krauss, IEEE J. Quantum Electron. 38 (2002) 915.

[184] T.F. Krauss, Phys. Stat. Sol. (a) (2003) 688.

[185] S. Noda, A. Chutinan, M. Imada, Nature 407 (2000) 608.

[186] B.S. Song, S. Noda, T. Asano, Science 300 (2003) 1537.

[187] Y. Akahane, T. I Asano, B.-S. Sond, S. Noda, Nature 425 (2003) 944.

[188] O. Painter, R.K. Lee, A. Scherer, A. Yariv, J.D. O’Brien, P.D. Dapkus, I. Kim, Science 284 (1999) 1819.

[189] H.-Y. Ryu, S.-H. Kim, H.-G. Park, J.-K. Hwang, Y.-H. Lee, J.-S. Kim, Appl. Phys. Lett. 80 (2002) 3883.

[190] T. Yoshie, O.B. Shchekin, H. Chen, D.G. Deppe, A. Scherer, Electron. Lett. 38 (2002) 967.

[191] H.-G. Park, S.-H. Kim, S.-H. Kwon, Y.-G. Ju, J.-K. Yang, J.-H. Baek, S.-B. Kim, Y.-H. Lee, Science 305 (2004) 1444.

[192] S.M. Spillane, T.J. Kippenberg, K.J. Vahala, Nature 415 (2002) 621.

[193] J. Vuckowic, M. Loncar, H. Mabuchi, A. Scherer, Phys. Rev. E 65 (2002) 16608.

[194] J.P. Reithmaier, G. Sk, A. Löffler, C. Hofmann, S. Kuhn, S. Reitzenstein, L.V. Keldysh, V.D. Kulakovskii, T.L. Reinecke, A. Forchel, Science 432 (2004) 197.

[195] T. Yoshie, A. Scherer, J. Hendrickson, G. Khitrova, H.M. Gibbs, G. Rupper, C. Ell, O.B. Shchekin, D.G. Deppe, Nature 432 (2004) 200.

[196] M.L. Gorodetsky, A.A. Savchenko, V.S. Ilchenko, Opt. Lett. 21 (1996) 453.

[197] A. Serpenguzel, S. Arnold, G. Griffel, Opt. Lett. 20 (1995) 654.

[198] G. Khitrova, H.M. Gibbs, F. Jahnke, S.W. Koch, Rev. Mod. Phys. 71 (1999) 1591.

[199] B. Song, S. Noda, T. Asano, Y. Akahane, Nat. Mater. 4 (2005) 207.

[200] Y. Akahane, T. Asano, B.-S. Song, S. Noda, Opt. Expr. 13 (2005) 1202.

[201] T. Asano, B.-S. Song, S. Noda, Opt. Expr. 14 (2006) 1996.

[202] E. Kuramochi, Workshop on technology issues in photonic crystals, in: PECS-VI, International Symposium on Photonic and Electromagnetic Crystal Structures, Aghia Pelaghia, Crete, Greece, 2005.

[203] Y. Vlasov, Workshop on technology issues in photonic crystals, in: PECS-VI, International Symposium on Photonic and Electromagnetic Crystal Structures, Aghia Pelaghia, Crete, Greece, 2005. 
[204] J.S. Jensen, O. Sigmund, L.H. Frandsen, P.I. Borel, A. Harpøth, M. Kristensen, IEEE Photonics Technol. Lett. 17 (6) (2005) 1041.

[205] M. Burger, S.J. Osher, E. Yablonovitch, IEICE Trans. Electron. E 87C (3) (2004) 258.

[206] M.P. Bendsœ, O. Sigmund, Topology Optimization—Theory, Methods and Applications, Springer, Berlin, 2003.

[207] H. Gersen, T.J. Karle, R.J.P. Engelen, W. Bogaerts, J.P. Korterik, N.F. von Hulst, T.F. Krauss, L. Kuipers, Phys. Rev. Lett. 94 (2005) 073903.

[208] S. Noda, K. Tomoda, N. Yamamoto, A. Chutinan, Science 289 (2000) 604.

[209] K.-M. Ho, C.T. Chan, C.M. Soukoulis, R. Biswas, M. Sigalas, Solid State Comm. 89 (1994) 413.

[210] S.Y. Lin, J.G. Fleming, D.L. Hetherington, B.K. Smith, R. Biswas, K.M. Ho, M.M. Sigalas, W. Zzubrzycki, S.R. Kurtz, J. Bur, Nature 394 (1998) 251.

[211] N. Yamamoto, S. Noda, A. Chutinan, Jpn. J. Appl. Phys. 37 (1998) L1052-4.

[212] J.G. Fleming, S.Y. Lin, Opt. Lett. 24 (1999) 49.

[213] S. Noda, N. Yamamoto, H. Kobayashi, M. Okano, K. Tomoda, Appl. Phys. Lett. 75 (1999) 905.

[214] S. Ogawa, M. Imada, S. Yoshimoto, M. Okana, S. Noda, Science 305 (2004) 227.

[215] G. Subramania, S.Y. Lin, Appl. Phys. Lett. 85 (2004) 5037.

[216] M. Qi, E. Lidorikis, P.T. Rakich, S.G. Johnson, J.D. Joannopoulos, E.I. Ippen, H.I. Smith, Nature 429 (2004) 538.

[217] S.G. Johnson, J.D. Joannopolous, Appl. Phys. Lett. 77 (2000) 3490.

[218] S. Matthias, F. Müller, C. Jamois, R.B. Wehrspohn, U. Gösele, Adv. Mater. 16 (2004) 2166.

[219] J. Schilling, J. White, A. Scherer, G. Stupian, R. Hillebrand, U. Gösele, Appl. Phys. Lett. 86 (2005) 11101.

[220] O. Toader, S. John, Science 292 (2001) 1133.

[221] K. Robbie, M.J. Brett, J. Vac. Soc. Technol. B 15 (1997) 1460.

[222] K. Robbie, J.C. Sit, M.J. Brett, J. Vac. Soc. Technol. B 16 (1998) 1115.

[223] S.R. Kennedy, M.J. Brett, O. Toader, S. John, Nano Lett. 2 (2002) 59.

[224] S.R. Kennedy, M.J. Brett, H. Miguez, O. Toader, S. John, Photonics Nanostruct. 1 (2003) 37.

[225] P.C.P. Hrudey, B. Szeto, M.J. Brett, Appl. Phys. Lett. 88 (2006) 251106.

[226] Y.K. Pang, J.C.W. Lee, H.F. Lee, W.Y. Tam, C.T. Chan, P. Sheng, Opt. Expr. 13 (2005) 7615.

[227] B.P.J., Curr. Sci. 76 (1999) 1311.

[228] P.N. Pusey, W. van Megen, Nature 320 (1998) 340.

[229] H. Miguez, F. Meseguer, C. Lopez, A. Blanco, J.S. Moya, J. Requena, A. Misfud, V. Fornes, Adv. Mater. 10 (1997) 480.

[230] A.S. Dimitrov, C.D. Dushkin, H. Yoshimura, K. Nagayama, Langmuir 10 (1994) 432.

[231] Yu.A. Vlasov, X.-Z. Bo, J.C. Strum, D.J. Norris, Nature 414 (2001) 289.

[232] P. Jiang, J.F. Bertone, K.S. Hwang, V.L. Colvin, Chem. Mater. 11 (1999) 2132.

[233] P. Jiang, G.N. Ostojic, R. Narat, D.M. Mittleman, V.L. Colvin, Adv. Mater. 13 (2001) 389.

[234] S. Wong, V. Kitaev, G.A. Ozin, J. Am. Chem. Soc. 125 (2003) 15589.

[235] T. Suzuki, P.K.L. Wu, J. Opt. Soc. Am. B 12 (1995) 570.

[236] R. Biswas, M. Sigalas, G. Subramania, K.-M. Ho, Phys. Rev. B 57 (1998) 3701.

[237] R. Biswas, M.M. Sigalas, G. Subramania, C.M. Soukoulis, K.-M. Ho, Phys. Rev. B 61 (2000) 4549.

[238] W. Lee, S. Pruzinsky, P. Braun, Adv. Mater. 14 (2002) 271.

[239] E. Vekris, V. Kitaev, G. von Freymann, D. Perovic, J.S. Aitchison, G.A. Ozin, Adv. Mater. 17 (2005) 1269.

[240] Q. Yan, Z. Zhou, X.S. Zhao, S.J. Chua, Adv. Mater. 17 (2005) 1917.

[241] A. van Blaaderen, R. Rue, P. Wiltzius, Nature 385 (1997) 321.

[242] H. Miguez, N. Tetreault, B. Hatton, S.M. Yang, D. Perovic, G.A. Ozin, Chem. Commun. 22 (2002) 2736.

[243] A. Blanco, E. Chomski, S. Grabtchak, M. Ibisate, S. John, S.W. Leonard, C. Lopez, F. Meseguer, H. Miguez, J.P. Mondia, G.A. Ozin, O. Toader, H.M. van Driel, Nature 405 (2000) 437.

[244] Y.A. Vlasov, X.Z. Bo, J.C. Sturm, D.J. Norris, Nature 414 (2001) 289.

[245] A.A. Chabanov, Y. Jun, D.J. Norris, Appl. Phys. Lett. 84 (2004) 3573.

[246] C. Lopez, Adv. Mater. 15 (2003) 1679.

[247] D.J. Norris, E.G. Arlinghaus, L.L. Meng, Adv. Mater. 16 (2004) 1393.

[248] N. Tétreault, H. Miguez, G.A. Ozin, Adv. Mater. 16 (2004) 1471.

[249] C.H.L. Goodman, M.V. Pessa, J. Appl. Phys. 60 (1986) R65.

[250] M. Ritala, M. Leskelä, Nanotechnology 10 (1999) 19.

[251] R.G. Gordon, Chem. Vap. Deposition 9 (2003) 73.

[252] L. Niistiö, J. Päiväsaari, J. Niinistö, M. Putkonen, M. Nieminen, Phys. Stat. Sol. (a) 201 (2004) 1443.

[253] F. Campbell, D.N. Sharp, M.T. Harrison, R.G. Denning, A.J. Turberfield, Nature 404 (2000) 53.

[254] S. Shoji, S. Kawata, Appl. Phys. Lett. 76 (2000) 2668.

[255] Yu.V. Miklyaev, D.C. Meisel, A. Blanco, G. von Freymann, K. Busch, W. Koch, C. Enkrich, M. Deubel, M. Wegener, Appl. Phys. Lett. 82 (2003) 1284.

[256] K.I. Petsas, A.B. Coates, G. Grynberg, Phys. Rev. A 50 (1994) 5173.

[257] K.K. Seet, S. Juodkazis, V. Jarutis, H. Misawa, Appl. Phys. Lett. 89 (2006) 024106.

[258] D.C. Meisel, M. Wegener, K. Busch, Phys. Rev. B 70 (2004) 165104.

[259] M. Maldovan, A.M. Urbas, N. Yufa, W.C. Carter, E.L. Thomas, Phys. Rev. B 65 (2002) 165123.

[260] O. Toader, T.Y.M. Chan, S. John, Phys. Rev. Lett. 92 (2004) 43905.

[261] C.K. Ullal, M. Maldovan, E.L. Thomas, G. Chen, Y.-J. Han, S. Yang, Appl. Phys. Lett. 84 (2004) 5434.

[262] C.K. Ullal, M. Maldovan, M. Wohlgemuth, E.L. Thomas, J. Opt. Soc. Am. A 20 (2003) 948. 
[263] V.P. Tondiglia, L.V. Natarajan, R.L. Sutherland, D. Tomlin, T.J. Bunning, Adv. Mater. 14 (2002) 187.

[264] S. Jeon, J.-U. Park, R. Cirelli, S. Yang, C.E. Heitzman, P.V. Braun, P.J.A. Kenis, J.A. Rogers, Proc. Natl. Acad. Sci. 101 (2004) 12428.

[265] Y. Lin, D. Rivera, K.P. Chen, Opt. Expr. 14 (2006) 887.

[266] T.Y.M. Chan, O. Toader, S. John, Phys. Rev. E 73 (2006) 046610.

[267] L. Wu, Y. Zhong, C.T. Chan, K.S. Wong, G.P. Wang, Appl. Phys. Lett. 86 (2005) 241102.

[268] H.-B. Sun, S. Matsuo, H. Misawa, Appl. Phys. Lett. 74 (1999) 786.

[269] S. Kawata, H.-B. Sun, T. Tanaka, K. Takada, Nature 412 (2001) 697.

[270] M. Straub, M. Gu, Opt. Lett. 27 (2002) 1824.

[271] M. Deubel, M. Wegener, A. Kaso, S. John, Appl. Phys. Lett. 85 (2004) 1895.

[272] K.K. Seet, V. Mizeikis, S. Matsuo, S. Juodkazis, H. Misawa, Adv. Mater. 17 (2005) 541.

[273] M. Thiel, M. Decker, M. Deubel, M. Wegener, S. Linden, G. von Freymann, Adv. Mater. 19 (2007) 207.

[274] A. Chutinan, S. John, O. Toader, Phys. Rev. Lett. 90 (2003) 123901.

[275] M. Deubel, M. Wegener, G. von Freymann, S. Linden, S. John, Opt. Lett. 31 (2006) 805.

[276] J.S. King, E. Graugnard, O.M. Roche, D.N. Sharp, C.J. Summers, R.G. Denning, A.J. Turberfield, Infiltration and inversion of holographicallydefined polymer photonic crystal templates using atomic layer deposition, PECS-VI, in: International Symposium on Photonic and Electromagnetic Crystal Structures, Aghia Pelaghia, Crete, Greece, 2005.

[277] N. Tétreault, G. von Freymann, M. Deubel, M. Hermatschweiler, F. Pérez-Willard, S. John, M. Wegener, G.A. Ozin, Adv. Mater. 18 (2006) 457.

[278] A.V. Kolobov, Photo-induced Metastability in Amorphous Semiconductors, Wiley-VCH, Weinheim, 2003.

[279] A. Feigel, Z. Kotler, B. Sfez, A. Arsh, M. Klebanov, V. Lyubin, Appl. Phys. Lett. 77 (2000) 3221.

[280] A. Feigel, M. Veinger, B. Sfez, A. Arsh, M. Klebanov, V. Lyubin, Appl. Phys. Lett. 83 (2003) 4480.

[281] S. Wong, M. Deubel, F. Pérez-Willard, S. John, G.A. Ozin, M. Wegener, G. von Freymann, Adv. Mater. 18 (2006) 265.

[282] Y.R. Shen, Principles of Nonlinear Optics, Wiley, New York, 1984.

[283] H.G. Winful, J.H. Marburger, E. Garmire, Appl. Phys. Lett. 35 (1979) 379.

[284] H.G. Winful, G.D. Cooperman, Appl. Phys. Lett. 40 (1982) 298.

[285] W. Chen, D.L. Mills, Phys. Rev. Lett. 58 (1987) 160.

[286] W. Chen, D.L. Mills, Phys. Rev. B 36 (1987) 6269.

[287] C.M. de Sterke, J.E. Sipe, Phys. Rev. A 39 (1989) 5163.

[288] C.M. de Sterke, J.E. Sipe, Prog. Opt. XXXIII (1994) 203.

[289] R.K. Dodd, J.C. Eilbeck, J.D. Gibbon, H.C. Morris, Solitons and Nonlinear Wave Equations, Academic Press, London, 1982.

[290] C.M. de Sterke, J.E. Sipe, Phys. Rev. A 38 (1988) 5149.

[291] J.E. Sipe, H.G. Winful, Opt. Lett. 13 (1988) 132.

[292] S. Pereira, J.E. Sipe, Phys. Rev. E 62 (2000) 5745.

[293] N.A.R. Bhat, J.E. Sipe, Phys. Rev. E 64 (2001) 056604.

[294] M. Bertolotti, J. Opt. A 8 (2006) S9.

[295] G.L. Fischer, R.W. Boyd, R.J. Gehr, S.A. Jenekhe, J.A. Osaheni, J.E. Sipe, A. Weller-Brophy, Phys. Rev. Lett. 74 (1995) 1871.

[296] L. Tkeshelashvili, S. Pereira, K. Busch, Europhys. Lett. 68 (2004) 205.

[297] L. Tkeshelashvili, J. Niegemann, S. Pereira, K. Busch, Photonics Nanostruct. 4 (2006) 75.

[298] B. Eiermann, Th. Anker, M. Albiez, M. Taglieber, P. Treutlein, K.-P. Marzlin, M.K. Oberthaler, Phys. Rev. Lett. 92 (2004) 230401.

[299] C.M. de Sterke, D.G. Salinas, J.E. Sipe, Phys. Rev. E 54 (1996) 1969.

[300] T. Iizuka, C.M. de Sterke, Phys. Rev. E 61 (2000) 4491.

[301] C.M. de Sterke, B.J. Eggleton, Phys. Rev. E 59 (1999) 1267.

[302] D.N. Christodoulides, R.I. Joseph, Phys. Rev. Lett. 62 (1989) 1746.

[303] A.B. Aceves, S. Wabnitz, Phys. Lett. A 141 (1989) 37.

[304] D.J. Kaup, A.C. Newell, Lett. Nouvo Cimento 20 (1977) 325.

[305] I.V. Barashenkov, D.E. Pelinovsky, E.V. Zemlyanaya, Phys. Rev. Lett. 80 (1998) 5117.

[306] A. De Rossi, C. Conti, S. Trillo, Phys. Rev. Lett. 81 (1998) 85.

[307] B.A. Malomed, R.S. Tasgal, Phys. Rev. E 49 (1994) 5787.

[308] C.M. de Sterke, Phys. Rev. A 45 (1992) 8252.

[309] T.I. Belova, A.E. Kudryavtsev, Physics-Uspekhi 40 (1997) 337.

[310] R.H. Goodman, R.E. Slusher, M.I. Weinstein, J. Opt. Soc. Am. B 19 (2002) 1635.

[311] W.C.K. Mak, B.A. Malomed, P.L. Chu, J. Opt. Soc. Am. B 20 (2003) 725.

[312] P.Y.P. Chen, B.A. Malomed, P.L. Chu, Phys. Rev. E 71 (2005) 066601.

[313] W.C.K. Mak, B.A. Malomed, P.L. Chu, Phys. Rev. E 67 (2003) 026608.

[314] N.G.R. Broderick, C.M. de Sterke, Phys. Rev. E 51 (1995) 4978.

[315] N.G.R. Broderick, C.M. de Sterke, Phys. Rev. E 58 (1998) 7941.

[316] C.M. de Sterke, J.E. Sipe, Phys. Rev. A 43 (1991) 2467.

[317] M.J. Steel, C.M. de Sterke, Phys. Rev. A 48 (1993) 1625.

[318] W.C.K. Mak, B.A. Malomed, P.L. Chu, Phys. Rev. E 68 (2003) 026609.

[319] N. Aközbek, S. John, Phys. Rev. E 57 (1998) 2287.

[320] B.J. Eggleton, R.E. Slusher, C.M. de Sterke, P.A. Krug, J.E. Sipe, Phys. Rev. Lett. 76 (1996) 1627.

[321] C.M. de Sterke, Phys. Rev. A 45 (1992) 2012. 
[322] M. Scalora, J.P. Dowling, C.M. Bowden, M.J. Bloemer, Phys. Rev. Lett. 73 (1994) 1368.

[323] C.M. de Sterke, J.E. Sipe, Phys. Rev. A 42 (1990) 2858.

[324] D. Taverner, N.G.R. Broderick, D.J. Richardson, R.I. Laming, M. Ibsen, Opt. Lett. 23 (1998) 328.

[325] D. Taverner, N.G.R. Broderick, D.J. Richardson, M. Ibsen, R.I. Laming, Opt. Lett. 23 (1998) 259.

[326] A.B. Aceves, Chaos 10 (2000) 584.

[327] B.I. Mantsyzov, Phys. Rev. A 51 (1995) 4939.

[328] B.I. Mantsyzov, JETP Lett. 82 (2005) 253.

[329] A. Kozhekin, G. Kurizki, Phys. Rev. Lett. 74 (1995) 5020.

[330] A. Kozhekin, G. Kurizki, B. Malomed, Phys. Rev. Lett. 81 (1998) 3647.

[331] T. Opatrny, B. Malomed, G. Kurizki, Phys. Rev. E 60 (1999) 6137.

[332] M. Blaauboer, G. Kurizki, B. Malomed, Phys. Rev. E 62 (2000) 57.

[333] N. Aközbek, S. John, Phys. Rev. E 58 (1998) 3876.

[334] B. Luo, H. Tseng, S. Chi, J. Opt. Soc. Am. B 20 (2003) 1866.

[335] B.I. Mantsyzov, I.V. Melnikov, J.S. Aitchison, Phys. Rev. E 69 (2004) 055602.

[336] I.V. Melnikov, J.S. Aitchison, Appl. Phys. Lett. 87 (2005) 201111.

[337] J. Zhou, H. Shao, J. Zhao, X. Yu, K.S. Wong, Opt. Lett. 30 (2005) 1560.

[338] N. Bloembergen, A.J. Sievers, Appl. Phys. Lett. 17 (1970) 483.

[339] J.P. Van der Ziel, M. Ilegem, Appl. Phys. Lett. 28 (1976) 437.

[340] J.A. Armstrong, N. Bloembergen, J. Ducuing, P.S. Pershan, Phys. Rev. 127 (1962) 1918.

[341] A. Fiore, V. Berger, E. Rosencher, P. Bravetti, J. Nagle, Nature 391 (1998) 463.

[342] F. Genereux, S.W. Leonard, H.M. van Driel, A. Birner, U. Gösele, Phys. Rev. B 63 (2001) R161101.

[343] A.V. Buryak, P. Di Trapani, D.V. Skryabin, S. Trillo, Phys. Rep. 370 (2002) 63.

[344] H. He, P.D. Drummond, Phys. Rev. Lett. 78 (1997) 4311.

[345] T. Peschel, U. Peschel, F. Lederer, B.A. Malomed, Phys. Rev. E 55 (1997) 4730.

[346] H. He, A. Arraf, C.M. de Sterke, P.D. Drummond, B.A. Malomed, Phys. Rev. E 59 (1999) 6064.

[347] C. Conti, S. Trillo, G. Assanto, Phys. Rev. Lett. 78 (1997) 2341.

[348] C. Conti, S. Trillo, G. Assanto, Opt. Lett. 22 (1997) 445.

[349] C. Conti, S. Trillo, G. Assanto, Phys. Rev. E 57 (1998) 1251.

[350] C. Conti, S. Trillo, G. Assanto, Opt. Lett. 23 (1998) 334.

[351] Y. Leitner, B.A. Malomed, Phys. Rev. E 71 (2005) 057601.

[352] C. Conti, G. Assanto, S. Trillo, Opt. Lett. 22 (1997) 1350.

[353] C. Conti, G. Assanto, S. Trillo, Phys. Rev. E 59 (1999) 2467.

[354] Y. Kivshar, Phys. Rev. E 51 (1995) 1613.

[355] C. Conti, A. De Rossi, S. Trillo, Opt. Lett. 23 (1998) 1265.

[356] T. Iizuka, C.M. de Sterke, Phys. Rev. E 62 (2000) 4246.

[357] S. Trillo, Opt. Lett. 21 (1996) 1732.

[358] A. Arraf, C.M. de Sterke, Phys. Rev. E 58 (1998) 7951.

[359] A. Arraf, C.M. de Sterke, H. He, Phys. Rev. E 63 (2001) 026611.

[360] L. Tkeshelashvili, K. Busch, Appl. Phys. B 81 (2005) 225.

[361] M.J.A. de Dood, W.T.M. Irvine, D. Bouwmeester, Phys. Rev. Lett. 93 (2004) 040504.

[362] W.T.M. Irvine, M.J.A. de Dood, D. Bouwmeester, Phys. Rev. A 72 (2005) 043815.

[363] D. Pezzetta, C. Sibilia, M. Bertolotti, J.W. Haus, M. Scalora, M.J. Bloemer, C.M. Bowden, J. Opt. Soc. Am. B 18 (2001) 1326.

[364] A.V. Balakin, V.A. Bushuev, B.I. Mantsyzov, I.A. Ozheredov, E.V. Petrov, A.P. Shkurinov, P. Masselin, G. Mouret, Phys. Rev. E 63 (2001) 046609 .

[365] Y. Dumeige, I. Sagnes, P. Monnier, P. Vidakovic, I. Abram, C. Meriadec, A. Levenson, Phys. Rev. Lett. 89 (2002) 043901.

[366] J.P. Mondia, H.M. van Driel, W. Jiang, A.R. Cowan, J.F. Young, Opt. Lett. 28 (2003) 2500.

[367] A.R. Cowan, J.F. Young, Semicond. Sci. Technol. 20 (2005) R41.

[368] P.P. Markowicz, H. Tiryaki, H. Pudavar, P.N. Prasad, N.N. Lepeshkin, R.W. Boyd, Phys. Rev. Lett. 92 (2004) 083903.

[369] P.P. Markowicz, V.K.S. Hsiao, H. Tiryaki, A.N. Cartwrite, P.N. Prasad, K. Dolgaleva, N.N. Lepeshkin, R.W. Boyd, Appl. Phys. Lett. 87 (2005) 05110.

[370] C. Becker, M. Wegener, S. Wong, G. von Freymann, Appl. Phys. Lett. 89 (2006) 131122.

[371] M. Nomura, S. Iwamoto, K. Watanabe, N. Kumagai, Y. Nakata, S. Ishida, Y. Arakawa, Opt. Expr. 14 (2006) 6308.

[372] S. Strauf, K. Hennessy, M.T. Rakher, Y.-S. Choi, A. Badolato, L.C. Andreani, E.L. Hu, P.M. Petroff, D. Bouwmeester, Phys. Rev. Lett. 96 (2006) 127404.

[373] H. Altug, D. Englund, J. Vuckovic, Nat. Phys. 2 (2006) 484.

[374] T.D. Happ, A. Markard, M. Kamp, J.L. Gentner, A. Forchel, J. Vac. Sci. Technol. B 19 (2001) 2775.

[375] T.D. Happ, A. Markard, M. Kamp, S. Anand, A. Forchel, Appl. Phys. Lett. 79 (2001) 4091.

[376] T.D. Happ, M. Kamp, A. Forchel, J.L. Gentner, L. Goldstein, Appl. Phys. Lett. 82 (2003) 4.

[377] A.R. Alija, L.J. Martinez, P.A. Postigo, C. Seassal, P. Viktorovitch, Appl. Phys. Lett. 89 (2006) 101102.

[378] B. Maune, J. Witzens, T. Baehr-Jones, M. Kolodrubetz, H. Atwater, A. Scherer, R. Hagen, Y. Qiu, Opt. Expr. 13 (2005) 4699.

[379] R. Colombelli, K. Srinivasan, M. Troccoli, O. Painter, C.F. Gmachl, D.M. Tennant, A.M. Sergent, D.L. Sivco, A.Y. Cho, F. Capasso, Science 302 (2003) 1374. 
[380] S. Riechel, C. Kallinger, U. Lemmer, J. Feldmann, A. Gombert, V. Wittwer, U. Scherf, Appl. Phys. Lett. 77 (2000) 2310.

[381] G. Turnbull, P. Andrew, W.L. Barnes, I.D.W. Samuel, Phys. Rev. B 67 (2003) 165107.

[382] K. Forberich, M. Diem, J. Crewett, U. Lemmer, A. Gombert, K. Busch, Appl. Phys. B 82 (2006) 539.

[383] S. John, J. Wang, Phys. Rev. Lett. 64 (1990) 2418.

[384] S. John, J. Wang, Phys. Rev. B 43 (1991) 12772.

[385] N. Vats, S. John, K. Busch, Phys. Rev. A 65 (2002) 043808.

[386] S. John, T. Quang, Phys. Rev. A 50 (1994) 1764.

[387] N. Vats, S. John, Phys. Rev. A 58 (1998) 4168.

[388] M. Woldeyohannes, S. John, Phys. Rev. A 60 (1999) 5046.

[389] B. Fain, Phys. Rev. A 37 (1988) 546.

[390] P. Lodahl, A.F. van Driel, I.S. Nikolaev, A. Irman, K. Overgaag, D. Vanmaekelbergh, W.L. Vos, Nature 430 (2004) 654.

[391] M. Fujita, S. Takahashi, Y. Tanaka, T. Asano, S. Noda, Science 308 (2005) 1296.

[392] M. Barth, R. Schuster, A. Gruber, F. Cichos, Phys. Rev. Lett. 96 (2006) 243902.

[393] J.B. Johnson, R.W. Christy, Phys. Rev. B 6 (1972) 4370.

[394] E.D. Palik, Handbook of Optical Constants of Solids, Academic Press, New York, 1985.

[395] C. Kittel, Quantum Theory of Solids, Wiley, New York, 1987.

[396] K.J. Chau, G.D. Dice, A.Y. Elezzabi, Phys. Rev. Lett. 94 (2005) 173904.

[397] G. Mie, Ann. Phys. 25 (1908) 377.

[398] D.F. Sievenpiper, M.E. Sickmiller, E. Yablonovitch, Phys. Rev. Lett. 76 (1996) 2480.

[399] J.G. Fleming, S.Y. Lin, I. El-Kady, R. Biswas, K.M. Ho, Nature 417 (2002) 52.

[400] S.Y. Lin, J. Moreno, J.G. Fleming, Appl. Phys. Lett. 83 (2004) 380.

[401] C.H. Seager, M.B. Sinclair, J.B. Fleming, Appl. Phys. Lett. 86 (2005) 244105.

[402] T. Trupke, P. Würfel, M.A. Green, Appl. Phys. Lett. 84 (2004) 1997.

[403] C. Luo, A. Narayanaswamy, G. Chen, J.D. Joannopoulos, Phys. Phys. Lett. 93 (2004) 213905.

[404] W.W. Chow, Phys. Rev. A 73 (2005) 013821.

[405] G. von Freymann, S. John, M. Schulz-Dobrick, E. Vekris, N. Tétreault, S. Wong, V. Kitaev, G.A. Ozin, Appl. Phys. Lett. 84 (2004) 224.

[406] T.W. Ebbesen, H.J. Lezec, H.F. Ghaemi, T. Thio, P.A. Wolff, Nature 391 (1998) 667.

[407] H.A. Bethe, Phys. Rev. 66 (1944) 163.

[408] C.J. Bouwkamp, Philipps Res. Rep. 5 (1950) 321.

[409] R.H. Richie, Phys. Rev. 106 (1957) 874.

[410] S.A. Maier, H.A. Atwater, J. Appl. Phys. 98 (2005) 11101.

[411] D.E. Grupp, H.J. Lezec, T.W. Ebbesen, K.M. Pellerin, T. Thio, Appl. Phys. Lett. 77 (2000) 1569.

[412] W.C. Tan, T.W. Preist, R.J. Sambles, Phys. Rev. B 62 (2000) 11134.

[413] E. Popov, M. Neviere, S. Enoch, R. Reinisch, Phys. Rev. B 62 (2000) 16100.

[414] L. Martin-Moreno, F.J. Garcia-Vidal, H.J. Lezec, K.J. Pellerin, T. Thio, J.B. Pendry, T.M. Ebbesen, Phys. Rev. Lett. 86 (2001) 1114.

[415] S.A. Darmanyan, A.V. Zayats, Phys. Rev. B 67 (2003) 35424.

[416] U. Schröter, D. Heitmann, Phys. Rev. B 60 (1999) 4992.

[417] I.R. Hooper, J.R. Sambles, Phys. Rev. B 67 (2003) 235404.

[418] H.J. Lezec, T. Thio, Opt. Expr. 12 (2004) 3629.

[419] K.J. Klein Koerkamp, S. Enoch, F.B. Segerink, N.F. van Hulst, L. Kuipers, Phys. Rev. Lett. 92 (2004) 183901.

[420] F. Falcone, T. Lopetegi, M.A.G. Laso, J.D. Baena, J. Bonache, M. Beruete, R. Marques, F. Martin, M. Sorella, Phys. Rev. Lett. 93 (2004) 197401.

[421] S. Linden, J. Kuhl, H. Giessen, Phys. Rev. Lett. 86 (2001) 4688.

[422] A. Christ, S.G. Tikhodeev, N.A. Gippius, J. Kuhl, H. Giessen, Phys. Rev. Lett. 91 (2003) 183901.

[423] S. Linden, N. Rau, U. Neuberth, A. Naber, M. Wegener, S. Pereira, K. Busch, A. Christ, J. Kuhl, Phys. Rev. B 71 (2005) 245119.

[424] M.W. Klein, T. Tritschler, M. Wegener, S. Linden, Phys. Rev. B 72 (2005) 115113.

[425] U. Kreibig, M. Vollmer, Optical Properties of Metal Clusters, Springer, Berlin, 1995.

[426] M. Nevière, Electromagnetic Theory of Gratings, Springer, Berlin, 1980, Chapter 5, pp. 123-157.

[427] T. Zentgraf, A. Christ, J. Kuhl, H. Giessen, Phys. Rev. Lett. 93 (2004) 243901.

[428] S. Linden, M. Decker, M. Wegener, Phys. Rev. Lett. 97 (2006) 083902.

[429] V.G. Veselago, Appl. Phys. B 81 (2005) 403.

[430] D.R. Smith, S. Schultz, P. Markos, C.M. Soukoulis, Phys. Rev. B 65 (2002) 195104.

[431] Th. Koschny, P. Markos, E.N. Economou, D.R. Smith, D.C. Vier, C.M. Soukoulis, Phys. Rev. B 71 (2005) 245105.

[432] L.V. Panina, A.N. Grigorenko, D.P. Makhnovskiy, Phys. Rev. B 66 (2002) 155411.

[433] G. Dolling, M. Wegener, S. Linden, C. Hormann, Opt. Expr. 14 (2006) 1842.

[434] J. Kästel, M. Fleischhauer, Laser Phys. 15 (2005) 1.

[435] R. Ruppin, Phys. Lett. A 277 (2000) 61.

[436] J.B. Pendry, Phys. Rev. Lett. 85 (2000) 3966.

[437] E. Shamonina, V.A. Kalinin, K.H. Ringhofer, L. Solymar, Electron. Lett. 37 (2001) 1243.

[438] D.R. Smith, D. Schurig, M. Rosenbluth, S. Schultz, S.A. Ramakrishna, J.B. Pendry, Appl. Phys. Lett. 82 (2003) 1506.

[439] R. Merlin, Appl. Phys. Lett. 84 (2004) 1290.

[440] V.A. Podolskiy, E.E. Narimanov, Opt. Lett. 30 (2005) 75. 
[441] A.N. Lagarkov, V.N. Kissel, Phys. Rev. Lett. 92 (2004) 077401.

[442] J.B. Pendry, S.A. Ramakrishna, Physica B 338 (2003) 329.

[443] N. Fang, H. Lee, C. Sun, X. Zhang, Science 308 (2005) 534.

[444] D.O.S. Melville, R.J. Blaikie, Opt. Expr. 13 (2005) 2127.

[445] I.V. Shadrivov, A.A. Sukhorukov, Y.S. Kivshar, Phys. Rev. Lett. 95 (2005) 193903.

[446] S.A. Ramakrishna, J.B. Pendry, Phys. Rev. B 69 (2004) 115115.

[447] J.B. Pendry, S.A. Ramakrishna, J. Phys.: Condens. Matter 15 (2003) 6345.

[448] U. Leonhardt, Science 312 (2006) 1777.

[449] J.B. Pendry, D. Schurig, D.R. Smith, Science 312 (2006) 1780.

[450] S.A. Cummer, B.-I. Popa, D. Schurig, D.R. Smith, J.B. Pendry, Phys. Rev. E 74 (2006) 036621.

[451] H.T. Dung, S.Y. Buhmann, L. Knöll, D.-G. Welsch, Phys. Rev. A 68 (2003) 43816.

[452] J. Kästel, M. Fleischhauer, Phys. Rev. A 1 (2005) 11804.

[453] S. O’Brien, D. McPeake, S.A. Ramakrishnan, J.B. Pendry, Phys. Rev. B 69 (2004) R241101.

[454] A.A. Zharov, I.V. Shadrivov, Y.S. Kivshar, Phys. Rev. Lett. 91 (2003) 037401-1.

[455] V.M. Agranovich, Y.R. Shen, R.H. Baughman, A.A. Zakhidov, Phys. Rev. B 69 (2004) 165112.

[456] A.A. Zharov, N.A. Zharova, I.V. Shadrivov, Y.S. Kivshar, Appl. Phys. Lett. 87 (2005) 091104.

[457] M.V. Gorkunov, I.V. Shadrivov, Y.S. Kivshar, Appl. Phys. Lett. 88 (2006) 071912.

[458] I.V. Shadrivov, A.A. Zharov, Y.S. Kivshar, J. Opt. Soc. Am. B 23 (2006) 1.

[459] G.D. Malyuzhinets, Zh. Tekh. Fiz. 21 (1951) 940.

[460] G.V. Eleftheriades, A.K. Iyer, P.C. Kremer, IEEE Trans. Microwave Theory Tech. 50 (2002) 2702.

[461] A. Lai, C. Caloz, T. Itoh, IEEE Microwave Mag. 4 (2004) 34.

[462] N. Engheta, A. Salandrino, A. Alú, Phys. Rev. Lett. 95 (2005) 095504.

[463] E.M. Lifshitz, L.D. Landau, L.P. Pitaevskii, Electrodynamics of Continuous Media, vol. 8, Pergamon Press, New York, 1960.

[464] W.N. Hardy, L.A. Whitehead, Rev. Sci. Instrum. 52 (1981) 213.

[465] J.B. Pendry, A.J. Holden, W.J. Stewart, I. Youngs, Phys Rev. Lett. 76 (1996) 4773.

[466] J.B. Pendry, A.J. Holden, D.J. Robbins, W.J. Stewart, IEEE Trans. Microwave Theory Tech. 47 (1999) 2075.

[467] H.J. Schneider, P. Dullenkopf, Rev. Sci. Instrum. 48 (1977) 68.

[468] B.T. Ghim, G.A. Rinard, W. Quine, S.S. Eaton, G.R. Eaton, J. Magn. Res. A 120 (1996) 72.

[469] S. Linden, C. Enkrich, M. Wegener, J. Zhou, T. Koschny, C.M. Soukoulis, Science 306 (2004) 1351.

[470] S. O’Brien, D. McPeake, S.A. Ramakrishnan, J.B. Pendry, Phys. Rev. B 69 (2004) R241101.

[471] J. Zhou, T. Koschny, M. Kafesaki, E.N. Economou, J.B. Pendry, C.M. Soukoulis, Phys. Rev. Lett. 95 (2005) 223902.

[472] A. Ishikawa, T. Tanaka, S. Kawata, Phys. Rev. Lett. 95 (2005) 237401.

[473] M.W. Klein, C. Enkrich, M. Wegener, C.M. Soukoulis, S. Linden, Opt. Lett. 31 (2006) 1259.

[474] E. Shamonina, V.A. Kalinin, K.H. Ringhofer, L. Solymar, J. Appl. Phys. 92 (2002) 6252.

[475] E. Shamonina, V.A. Kalinin, K.H. Ringhofer, L. Solymar, Electron. Lett. 38 (2002) 371.

[476] O. Sydoruk, O. Zhuromskyy, E. Shamonina, L. Solymar, Appl. Phys. Lett. 87 (2005) 072501.

[477] M.C.K. Wiltshire, E. Shamonina, I.R. Young, L. Solymar, Electron. Lett. 39 (2003) 215.

[478] G. Dolling, M. Wegener, A. Schädle, S. Burger, S. Linden, Appl. Phys. Lett. 89 (2006) 231118.

[479] M.V. Gorkunov, S.A. Gredesku, I.V. Shadrivov, Y.S. Kivshar, Phys. Rev. E 73 (2006) 056605.

[480] I.A. Larkin, M. Stockman, Nano Lett. 5 (2005) 339.

[481] R.A. Shelby, D.R. Smith, S. Schultz, Science 292 (2001) 77.

[482] T.J. Yen, W.J. Padilla, N. Fang, D.C. Vier, D.R. Smith, J.B. Pendry, D.N. Basov, X. Zhang, Science 303 (2004) 1494.

[483] D.R. Smith, J.B. Pendry, M.C.K. Wiltshire, Science 305 (2004) 788.

[484] N. Katsarakis, T. Koschny, M. Kafesaki, E.N. Economou, C.M. Soukoulis, Appl. Phys. Lett. 84 (2004) 2943.

[485] C. Enkrich, F. Pérez-Willard, D. Gerthsen, J. Zhou, C.M. Soukoulis, M. Wegener, S. Linden, Adv. Mater. 17 (2005) 2547.

[486] C. Rockstuhl, T. Zentgraf, H. Guo, N. Liu, C. Etrich, I. Loa, K. Syassen, J. Kuhl, F. Lederer, H. Giessen, Appl. Phys. B 84 (2006) 219.

[487] V.A. Podolskiy, A.K. Sarychev, E.E. Narimanov, V.M. Shalaev, J. Opt. A: Pure Appl. Opt. 7 (2005) 32.

[488] C. Enkrich, M. Wegener, S. Linden, S. Burger, L. Zschiedrich, F. Schmidt, J. Zhou, T. Koschny, C.M. Soukoulis, Phys. Rev. Lett. 95 (2005) 203901.

[489] M. Shamonin, E. Shamonina, V. Kalinin, L. Solymar, J. Appl. Phys. 95 (2004) 3778.

[490] M.W. Klein, C. Enkrich, M. Wegener, C.M. Soukoulis, S. Linden, Opt. Lett. 31 (2006) 1259.

[491] M.W. Klein, C. Enkrich, M. Wegener, S. Linden, Science 313 (2006) 502.

[492] G. Dolling, C. Enkrich, M. Wegener, J. Zhou, C.M. Soukoulis, S. Linden, Opt. Lett. 30 (2005) 3198.

[493] A.N. Lagarkov, A.K. Sarychev, Phys. Rev. B 53 (1996) 6318.

[494] V.M. Shalaev, W. Cai, U.K. Chettiar, H. Yuan, A.K. Sarychev, V.P. Drachev, A.V. Kildishev, Opt. Lett. 30 (2005) 3356.

[495] N. Feth, C. Enkrich, M. Wegener, S. Linden, Opt. Expr. 15 (2007) 501.

[496] Y. Liu, C. Sun, X. Zhang, E. Kim, Y.R. Shen, N.X. Fang, Appl. Phys. Lett. 90 (2007) 063107.

[497] F. Garwe, C. Rockstuhl, C. Etrich, U. Hübner, U. Bauerschäfer, F. Setzpfand, M. Augustin, T. Pertsch, A. Tünnermann, F. Lederer, Appl. Phys. B 84 (2006) 139.

[498] A.N. Grigorenko, A.K. Geim, H.F. Gleeson, Y. Zhang, A.A. Firsov, I.Y. Khrushchev, J. Petrovic, Nature 438 (2005) 335.

[499] G. Dolling, C. Enkrich, M. Wegener, C.M. Soukoulis, S. Linden, Science 312 (2006) 892.

[500] G. Dolling, C. Enkrich, M. Wegener, C.M. Soukoulis, S. Linden, Opt. Lett. 31 (2006) 1800. 
[501] G. Dolling, M. Wegener, C.M. Soukoulis, S. Linden, Opt. Lett. 32 (2007) 53.

[502] S. Zhang, W. Fan, K.J. Malloy, S.R.J. Brueck, N.C. Panoiu, R.M. Osgood, Opt. Expr. 13 (2005) 4922.

[503] S. Zhang, W. Fan, B.K. Minhas, A. Frauenglass, K.J. Malloy, S.R.J. Brueck, Phys. Rev. Lett. 94 (2005) 037402.

[504] C.M. Soukoulis, S. Linden, M. Wegener, Science 315 (2007) 47.

[505] V.M. Shalaev, Nat. Photonics 1 (2007) 41.

[506] A.K. Popov, V.M. Shalaev, Opt. Lett. 31 (2006) 2169.

[507] S. Zhang, W. Fan, N.C. Panoiu, K.J. Malloy, R.M. Osgood, S.R.J. Brueck, Opt. Expr. 14 (2006) 6778.

[508] G. Dolling, M. Wegener, S. Linden, Opt. Lett. 32 (2007) 551.

[509] X. Chen, B.-I. Wu, J.A. Kong, T.M. Grzegorczyk, Phys. Rev. E 71 (2005) 46610.

[510] J.B. Pendry, Science 306 (2004) 1353.

[511] Y. Svirko, N. Zheludev, M. Osipov, Appl. Phys. Lett. 78 (2003) 498.

[512] A. Papakostas, A. Potts, D.M. Bagnall, S.L. Prosvirnin, H.J. Coles, N.I. Zheludev, Phys. Rev. Lett. 90 (2003) 107404.

[513] A.S. Schwanecke, A. Krasavin, D.M. Bagnall, A. Potts, A.V. Zayats, N.I. Zheludev, Phys. Rev. Lett. 91 (2003) 247404.

[514] M. Kuwata-Gonokami, N. Saito, Y. Ino, M. Kauranen, K. Jefimovs, T. Vallius, J. Turunen, Y. Svirko, Phys. Rev. Lett. 95 (2005) 227401.

[515] M. Reichelt, S.W. Koch, A.V. Krasavin, J.V. Moloney, A.S. Schwanecke, T. Stroucken, E.M. Wright, N.I. Zheludev, Appl. Phys. B 84 (2006) 97.

[516] M. Decker, M.W. Klein, M. Wegener, S. Linden, Opt. Lett. 32 (2007) 856. 